\title{
A STUDY OF MUON NEUTRINO TO ELECTRON NEUTRINO OSCILLATIONS IN THE MINOS EXPERIMENT
}

\author{
A DISSERTATION \\ SUBMITTED TO THE DEPARTMENT OF PHYSICS \\ AND THE COMMITTEE ON GRADUATE STUDIES \\ OF STANFORD UNIVERSITY \\ IN PARTIAL FULFILLMENT OF THE REQUIREMENTS \\ FOR THE DEGREE OF \\ DOCTOR OF PHILOSOPHY
}

Tingjun Yang

March 2009 
(C) Copyright by Tingjun Yang 2009

All Rights Reserved 
I certify that I have read this dissertation and that, in my opinion, it is fully adequate in scope and quality as a dissertation for the degree of Doctor of Philosophy.

(Stanley Wojcicki) Principal Adviser

I certify that I have read this dissertation and that, in my opinion, it is fully adequate in scope and quality as a dissertation for the degree of Doctor of Philosophy.

(Patricia Burchat)

I certify that I have read this dissertation and that, in my opinion, it is fully adequate in scope and quality as a dissertation for the degree of Doctor of Philosophy.

(Giorgio Gratta)

Approved for the University Committee on Graduate Studies. 


\section{Abstract}

The observation of neutrino oscillations (neutrino changing from one flavor to another) has provided compelling evidence that the neutrinos have non-zero masses and that leptons mix, which is not part of the original Standard Model of particle physics. The theoretical framework that describes neutrino oscillation involves two mass scales $\left(\Delta m_{\text {atm }}^{2}\right.$ and $\left.\Delta m_{\text {sol }}^{2}\right)$, three mixing angles $\left(\theta_{12}, \theta_{23}\right.$, and $\left.\theta_{13}\right)$ and one $\mathrm{CP}$ violating phase $\left(\delta_{C P}\right)$. Both mass scales and two of the mixing angles $\left(\theta_{12}\right.$ and $\left.\theta_{23}\right)$ have been measured by many neutrino experiments. The mixing angle $\theta_{13}$, which is believed to be very small, remains unknown. The current best limit on $\theta_{13}$ comes from the CHOOZ experiment: $\theta_{13}<11^{\circ}$ at $90 \%$ C.L. at the atmospheric mass scale. $\delta_{C P}$ is also unknown today.

MINOS, the Main Injector Neutrino Oscillation Search, is a long baseline neutrino experiment based at Fermi National Accelerator Laboratory. The experiment uses a muon neutrino beam, which is measured $1 \mathrm{~km}$ downstream from its origin in the Near Detector at Fermilab and then $735 \mathrm{~km}$ later in the Far Detector at the Soudan mine. By comparing these two measurements, MINOS can obtain parameters in the atmospheric sector of neutrino oscillations. MINOS has published results on the precise measurement of $\Delta m_{\text {atm }}^{2}$ and $\theta_{23}$ through the disappearance of muon neutrinos in the Far Detector and on a search for sterile neutrinos by looking for a deficit in the number of neutral current interactions seen in the Far Detector. MINOS also has the potential to improve the limit on the neutrino mixing angle $\theta_{13}$ or make the first 
measurement of its value by searching for an electron neutrino appearance signal in the Far Detector. This is the focus of the study presented in this thesis.

We developed a neural network based algorithm to distinguish the electron neutrino signal from background. The most important part of this measurement is the background estimation, which is done through extrapolation. The number of background events is measured at the Near Detector, then extrapolated to the Far Detector. Since different background sources extrapolate differently, some knowledge about the relative contribution from different background sources is necessary. We developed a method that can be used to obtain relative contributions of various background sources from comparison of background rates in the horn-on and horn-off configurations. We also described our effort to improve two aspects of the Monte Carlo simulation which are very important for the $\nu_{e}$ appearance analysis: one is the hadronization model in the neutrino-nucleon interactions, the other is the modeling of PMT crosstalk. We performed a blind analysis and examined several sidebands before looking at the signal region. After we opened the box, we observed a $1.4 \sigma$ excess of $\nu_{e}$-like events in the Far Detector compared with the number of predicted background events. The excess is well within the statistical fluctuation of the background events. If we interpret the excess as a $\nu_{e}$ signal from $\nu_{\mu} \rightarrow \nu_{e}$ oscillation, the best fit $\sin ^{2} 2 \theta_{13}$ value is consistent with the CHOOZ limit. However we want to emphasize that our result is consistent with $\theta_{13}=0$ at $90 \%$ C.L.. 


\section{Acknowledgements}

I would first and foremost like to thank my advisor, Stanley Wojcicki, for his exceptional advice and guidance throughout my $\mathrm{PhD}$. I have been fortunate to have the opportunity of working with such a good physicist. Stan taught me an appreciation for the physics, an attention to details, a dedication to my work and every skill that I have as an experimentalist. I am deeply indebted to him.

I also would like to thank my colleagues at Stanford, Hyejoo Kang, Simona Murgia, Sergei Avvakumov, Greg Pawloski, and George Irwin, who have always been very supportive of me. I enjoyed working with them on all kinds of interesting topics, including electronics, software, detector calibration, and data analysis. I had a lot of fun with Greg after he became my office mate. He is an excellent physicist and has a good sense of humor. I benefited a lot from the discussions with him. He also kindly generated a lot of special Monte Carlo files for my thesis work.

I am grateful to many people at Tufts University, especially to Hugh Gallagher and Tony Mann. I went to Tufts in the summer of 2006 to work on the hadronization model with Hugh and Costas. This turned out to be a really fantastic experience for me. I was really impressed by their solid knowledge of physics and amazing programming skills and I learned a lot of things from them. I am also greatly indebted to Tony for helping me understand many issues in the bubble chamber experiments.

I would like to thank many people at Fermilab who helped me a lot during my stay at Fermilab for the past several years. I am grateful to Niki Saoulidou whose 
enthusiasm in physics always inspires me. I am lucky to be in the office next to Robert Hatcher's. Despite the fact that he is a very busy person, he always helped me solve all kinds of software problems with great patience whenever I turned to him for help. Rob Plunkett has always been very supportive and encouraging to me. I had a lot of wonderful discussions with Adam Para when his office was still on the 12th floor. I had a lot of good times with Brian Rebel, Alysia Marino (when she was on MINOS), Panos Stamoulis (when he was at Fermilab), and Peter Shanahan, who also taught me a lot about the Near Detector.

I was at the Soudan mine in the summer of 2003 where I first met Debdatta Bhattacharya. We have been good friends over the past several years. I am also grateful to Louie Barrett for teaching me so many things about MINOS detectors and to Dave Saranen for teaching me how to use Linux.

I am greatly grateful to the fellow members of the $\nu_{e}$ appearance analysis group, especially to Anna, Alex, Chris, David Jaffe, Josh, Mayly, Milind, Pedro and Trish, for being such excellent people to work together with.

I want to thank Jiajie Ling for his close friendship and willingness to help at my difficult times.

The loving support and encouragement of my mother has been totally invaluable over the years. Finally, a very special thanks to my dear wife Fang for her love, support and patience over the past years and without whom I would not be able to finish my thesis. 


\section{Contents}

Abstract $\quad$ v

Acknowledgements vii

1 Introduction 1

1.1 A Brief History of Neutrinos . . . . . . . . . . . . . . . . . 1

1.2 The Standard Electro-Weak Interaction Model . . . . . . . . . . . . . 6

1.2.1 Gauge Theories . . . . . . . . . . . . . 6

1.2.2 Spontaneous Symmetry Breaking . . . . . . . . . . . . . . . 10

1.3 Massive Neutrinos . . . . . . . . . . . . . . . . . . . 20

1.3.1 Dirac and Majorana Masses . . . . . . . . . . . . . . 21

1.3.2 Seesaw Mechanism and Neutrino Mixing . . . . . . . . . . 23

1.4 Neutrino Experiments . . . . . . . . . . . . . . . . . . . . . 32

1.4.1 The Solar Neutrino Problem . . . . . . . . . . . . . . . . 33

1.4.2 The Atmospheric Neutrino Anomaly . . . . . . . . . . . . . 36

1.4.3 The 13 Sector . . . . . . . . . . . . . . . . 44

1.4.4 The LSND Result and MiniBooNE . . . . . . . . . . . . . . . 49

1.4.5 Neutrinoless Double $\beta$ Decay . . . . . . . . . . . . . . . 51

1.4.6 Kinematic tests for $\nu$ masses . . . . . . . . . . . . . 52

1.4.7 Summary . . . . . . . . . . . . . . . 53 
2 The MINOS Experiment $\quad 55$

2.1 The Fermilab Accelerator . . . . . . . . . . . . . . . 56

2.2 The NuMI Neutrino Beam . . . . . . . . . . . . . . . . . . 60

2.3 The MINOS Detectors . . . . . . . . . . . . . . . 62

2.3.1 MINOS Detector Technologies . . . . . . . . . . . . 63

2.3.2 The Far Detector . . . . . . . . . . . . . . . . 67

2.3.3 The Near Detector . . . . . . . . . . . . . . . . 71

2.4 Detector Calibration . . . . . . . . . . . . . 75

2.4.1 Relative Calibration .............. . . 75

2.4.2 Absolute Calibration . . . . . . . . . . . . . . 80

2.5 Monte Carlo Simulation . . . . . . . . . . . . . . . . . 82

2.6 MINOS Physics Analyses . . . . . . . . . . . . . . . 84

2.6.1 $\nu_{\mu}$ disappearance measurement . . . . . . . . . . . 85

2.6.2 Search for Neutral Current Disappearance . . . . . . . . . . 86

2.6.3 Search for $\nu_{e}$ Appearance . . . . . . . . . . . 86

3 MINOS $\nu_{\mu}$ to $\nu_{e}$ oscillation Search $\quad 87$

4 Electron Neutrino Identification $\quad 96$

4.1 CalDet Electrons and Pions . . . . . . . . . . . . . . 96

4.2 MINOS Electron Neutrino Identification . . . . . . . . . . . . 107

4.2.1 Overview .......................... 107

4.2 .2 Pre-selection Cuts . . . . . . . . . . . . . . . . 109

4.2 .3 PID Variables . . . . . . . . . . . . . . . . 111

4.2.4 Constructing ANN . . . . . . . . . . . . . 115

4.3 Near Detector Data Monte Carlo Comparisons . . . . . . . . . . . . . 120

4.4 Summary . . . . . . . . . . . . . . . . . . . 132 
5 MINOS Hadronization Model 133

5.1 Introduction . . . . . . . . . . . . . . . . . . . 133

5.2 The AGKY Model . . . . . . . . . . . . . . . . . . . . 134

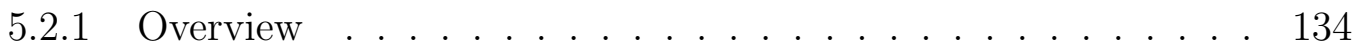

5.2.2 Low- $W$ model: Particle content . . . . . . . . . . . . . . . 137

5.2.3 Low- $W$ model: Hadron system decay . . . . . . . . . . . . . . 139

5.2.4 High- $W$ model: PYTHIA/JETSET . . . . . . . . . . . . . . . 141

5.3 Data/MC Comparisons . . . . . . . . . . . . . . . . . . . . 142

5.4 Intranuclear Rescattering . . . . . . . . . . . . . . . . 154

5.5 Conclusions . . . . . . . . . . . . . . . . . . . 156

6 Crosstalk Tuning $\quad 157$

6.1 Introduction . . . . . . . . . . . . . . . . . . . 157

6.2 Impact on the $\nu_{e}$ Analysis . . . . . . . . . . . . . . . 159

6.3 PMT Crosstalk Tuning . . . . . . . . . . . . . . . . . . 163

6.4 Changes to the $\nu_{e}$ analysis $\ldots \ldots \ldots \ldots$. . . . . . . . 186

6.5 Summary . . . . . . . . . . . . . . . . . . . 189

7 Near Detector Background Decomposition 190

7.1 Introduction . . . . . . . . . . . . . . . . . . . . . . . 190

7.2 Horn-on and Horn-off Spectra . . . . . . . . . . . . . . . . 191

7.3 ND $\nu_{e}$ Background Decomposition . . . . . . . . . . . . . . . . . 195

7.4 Background Systematics . . . . . . . . . . . . . . 198

7.4.1 Data (Horn-on/off) Statistics . . . . . . . . . . . . . 199

7.4.2 Beam $\nu_{e}$ Background . . . . . . . . . . . . . 199

7.4.3 Uncertainties in the Horn-off/on Ratios . . . . . . . . . . . . . 201

7.4.4 Calculation of the ND NC and CC Background Energy Spectra 211

7.5 Summary . . . . . . . . . . . . . . . . . . 217 
8 Sensitivity to $\theta_{13} \quad 218$

8.1 FD Background Prediction . . . . . . . . . . . . . . 218

8.2 Background Systematic Errors . . . . . . . . . . . . . . 221

8.2.1 Uncertainties in NC and $\nu_{\mu}$ CC Predictions . . . . . . . . . 221

8.2.2 Uncertainties in the beam $\nu_{e}$ and $\nu_{\tau}$ backgrounds . . . . . . 231

8.3 Signal Systematic Errors . . . . . . . . . . . . . . . . 235

8.4 MINOS Sensitivity to $\theta_{13} \ldots \ldots \ldots \ldots . \ldots \ldots$

9 First Results of MINOS $\nu_{e}$ Analysis $\quad 241$

9.1 Blind Analysis and Sidebands . . . . . . . . . . . . . . . . . . 241

9.2 Far Detector Data . . . . . . . . . . . . . . . . . . . . 242

9.3 Fiducial Volume Cuts . . . . . . . . . . . . . . . . . . . . . 244

9.4 Pre-selection Cuts . . . . . . . . . . . . . . . . . 247

9.5 PID Cuts . . . . . . . . . . . . . . . . . 249

9.6 Discussion of our results . . . . . . . . . . . . . . . 256

$\begin{array}{ll}\text { A Glossary of Acronyms } & 258\end{array}$

$\begin{array}{ll}\text { Bibliography } & 260\end{array}$ 


\section{List of Tables}

1.1 Neutral vector and axial vector couplings in the Standard Model . . .

1.2 The current state of knowledge of the neutrino parameters. . . . . . 53

4.1 Summary of Far Detector data reduction. The oscillation parameters are $\left|\Delta m_{31}^{2}\right|=2.4 \times 10^{-3} \mathrm{eV}^{2}, \sin ^{2} 2 \theta_{13}=0.15$ (CHOOZ limit), $\sin ^{2} 2 \theta_{23}=1$. We assume POT $=3.25 \times 10^{20}$ (2-year exposure). FOM is defined as $N^{s i g} / \sqrt{N^{b g}+\left(10 \% \times N^{b g}\right)^{2}} \ldots \ldots \ldots \ldots 119$

4.2 Numbers of accepted events at both detectors for MC with default intranuke model (Def.) and MC with the corrected intranuke model (INu.). The oscillation parameters are $\left|\Delta m_{31}^{2}\right|=2.4 \times 10^{-3} \mathrm{eV}^{2}, \sin ^{2} 2 \theta_{13}=$ $0.15, \sin ^{2} 2 \theta_{23}=1$. The numbers are normalized to $\mathrm{FD} \mathrm{POT}=3.25 \times$ $10^{20}$ (2-year exposure) and ND POT $=1 \times 10^{19}$. The errors are only statistical errors. . . . . . . . . . . . . . . . . . . 131

5.1 Default AGKY configuration parameters (see text for details) $\ldots . \quad 139$ 
6.1 Numbers of accepted events at both detectors for MC with default crosstalk fractions (Def) and MC with the new crosstalk fractions (NXT). The oscillation parameters are $\left|\Delta m_{31}^{2}\right|=2.4 \times 10^{-3} \mathrm{eV}^{2}, \sin ^{2} 2 \theta_{13}=$ $0.15, \sin ^{2} 2 \theta_{23}=1$. The numbers are normalized to FD POT $=3.25 \times$ $10^{20}$ (2-year exposure) and ND POT $=1 \times 10^{19}$. The errors are only statistical errors. . . . . . . . . . . . . . . . . . . . . . 188

7.1 Number of events that are accepted as $\nu_{e}$ candidates. Top: all events, comparison of data and MC; Middle: $\mathrm{MC}$ events $E_{\nu}<10 \mathrm{GeV}$; Bottom: $\mathrm{MC}$ events $E_{\nu}>10 \mathrm{GeV}$. All numbers represent $1 \times 10^{19}$ POTs

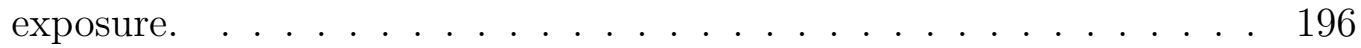

7.2 Percentage systematic errors on $r_{N C}$ in reconstructed energy bins. . . 212

7.3 Percentage systematic errors on $r_{C C}$ in reconstructed energy bins. . . 212

7.4 Percentage systematic errors on $r_{e}$ in reconstructed energy bins. . . . 213

7.5 The ND background estimates from the comparison of horn-off to hornon data and the ND background estimates from the Monte Carlo prediction assuming $15 \%$ beam $\nu_{e}$ error. . . . . . . . . . . . 215

7.6 Contributions to the ND background NC error assuming $15 \%$ beam $\nu_{e}$ error. Note total statistical errors are smaller than the individual statistical errors added in quadrature because of truncation applied to highest energy bin. . . . . . . . . . . . . . . . . . 216

7.7 Contributions to the ND background CC error assuming $15 \%$ beam $\nu_{e}$ error. Note total statistical errors are smaller than the individual statistical errors added in quadrature because of truncation applied to highest energy bin. . . . . . . . . . . . . . . . . 216

$8.1 \quad$ FD background prediction $\left(3.25 \times 10^{20}\right.$ POTs $) \ldots \ldots \ldots \ldots \ldots$ 
8.2 Systematic errors from extrapolation on $\mathrm{NC}$ and $\nu_{\mu} \mathrm{CC}$ background events selected by ANN PID. . . . . . . . . . . . . . . . . . . 232

8.3 Systematic errors on the beam $\nu_{e}$ background events selected by ANN PID in Near and Far Detector Monte Carlo. . . . . . . . . . . . . . . 234

8.4 Systematic errors on the signal $\nu_{e}$ events selected by ANN. . . . . . . 237

9.1 FD data and MC reduction for $3.14 \times 10^{20}$ POTs. The standard MC was used to predict the number of background and potential signal events (i.e. no corrections based on ND data). Signal is computed at $\sin ^{2} 2 \theta_{13}=0.15$ (CHOOZ limit). . . . . . . . . . . . . . . . 248

9.2 Comparison of the data selected in the two PID regions compared to the raw BG MC, the corrected background prediction, and the potential signal. Signal is computed at $\sin ^{2}\left(2 \theta_{13}\right)=0.15$ (CHOOZ limit). Numbers are for $3.14 \times 10^{20}$ POTs . . . . . . . . . . . . . . . . . 249

9.3 Comparison of the data selected in the signal regions compared to the raw $\mathrm{BG} \mathrm{MC}$, the corrected background prediction, and the potential signal. Signal is computed at $\sin ^{2}\left(2 \theta_{13}\right)=0.15$ (CHOOZ limit). Numbers are for $3.14 \times 10^{20}$ POTs . . . . . . . . . . . . . 252 


\section{List of Figures}

1.1 The two possible mass hierarchies for the three known neutrino flavors. Plot taken from $[37] \ldots \ldots$

1.2 The SNO and Super-K results expressed as a measurement of the flux of $\mu+\tau$ neutrinos versus the flux of electron neutrinos. Plot taken from $[40] . \ldots \ldots \ldots \ldots$

1.3 (a) Neutrino oscillation parameter allowed region from KamLAND anti-neutrino data (shaded regions) and solar neutrino experiments (lines). (b) Result of a combined two-neutrino oscillation analysis of KamLAND and the observed solar neutrino fluxes under the assumption of CPT invariance. Plot taken from $[46] . \ldots . . . . . .$.

1.4 Ratio of muon-like neutrino events to electron-like neutrino events, divided by their respective Monte Carlo simulation value. Plot taken from $[49] . \ldots \ldots \ldots \ldots$

1.5 A sketch showing the relation between zenith angle and the distance traveled by atmospheric neutrinos (plot taken from [51]). $\cos \theta<0$ represents upward-going particles and $\cos \theta>0$ represents downwardgoing particles. The flight path of atmosphere neutrinos from production to detector extends from $\sim 10 \mathrm{~km}$ to $12,000 \mathrm{~km} . \quad \ldots \ldots . . . .40$ 
1.6 Zenith angle distributions of $\mu$-like and $e$-like events for sub-GeV and multi-GeV data sets. Upward-going particles have $\cos \Theta<0$ and downward-going particles have $\cos \Theta>0$. The bars show the MC no-oscillation prediction with statistical errors, and the line shows the best-fit expectation for $\nu_{\mu} \rightarrow \nu_{\tau}$ oscillations. Plots taken from [52]. . .

1.7 Ratio of the data to the MC events without neutrino oscillation (points) as a function of the reconstructed $L / E$ together with the best-fit expectation for 2-flavor $\nu_{\mu} \rightarrow \nu_{\tau}$ oscillations (solid line). Also shown are the best-fit expectation for neutrino decay (dashed line) and neutrino decoherence (dotted line). Plot taken from [54]. . . . . . . . . . . .

1.8 The new MINOS best fit point (black point) and the $68 \%$ and $90 \%$ CL contours. Overlaid are the 90\% CL contours from the Super-K zenith angle [53] and $L / E[54]$ analyses, and that from the $\mathrm{K} 2 \mathrm{~K}$ experiment [56]. Plot taken from [59]. . . . . . . . . . . . . .

1.9 The $90 \%$ CL exclusion regions for the Chooz experiment and the Palo Verde experiment. "Swap" and "Reactor Power" represent two analysis methods used in the Palo Verde experiment. Plot taken from [62]. . . 46

1.10 Current state of knowledge of the 13 Sector. Plot taken from [66]. . . 47

1.11 The region of oscillation parameter psace excluded at 90\% CL by the MiniBooNE result. Also shown are the regions allowed by the LSND result at $90 \% \mathrm{CL}$ and $95 \% \mathrm{CL}$, and the $90 \%$ exclusion contours of the KARMEN2 [75] and Bugey [76] experiments. Plot taken from [74]. . 50

1.12 Neutrinoless double beta decay. Plot taken from [33]. . . . . . . . . 51

2.1 The Fermilab accelerators. Those used in generation of the NuMI beam are the Linac, Booster, and Main Injector. Plot taken from [85]. . . . 57 
2.2 Injection of the Booster beam in the Main Injector as a function of time. Overlaid on top of each other at two traces: the MI charge (green) that increases 11 times in 11 separate injections from the Booster and the MI beam momentum (red) that is defined by the magnet currents, it ramps from 8 to $120 \mathrm{GeV} / c$, then ramps down to $8 \mathrm{GeV} / c$. . . . . 58

2.3 Plan and elevation views of the NuMI beam facility. A proton beam is directed onto a target, from which the secondary pions and kaons are focused into a decay volume via magnetic horns. Ionization chambers at the end of the beam line measure the uninteracted primary beam, secondary hadron beam and tertiary muon beam. Plot taken from [88]. 61

2.4 Calculated rate of $\nu_{\mu}$ charged-current interactions in the MINOS Near Detector. Three spectra are shown, corresponding to the low, medium, and high neutrino energy positions of the target. In these configurations, the target is located 10, 100, and $250 \mathrm{~cm}$ upstream of its fullyinserted position. Plot taken from [58]. . . . . . . . . . . . . . . 62

2.5 Cutaway drawing of a single scintillator strip. Light produced by ionizing particle is multiply reflected inside the strip by the outer reflective coating. Light absorbed by a WLS fiber is re-emitted isotropically. Those resulting photons whose directions fall within the total internal reflection cones are transported along the fiber to the edges of the detector, subsequently being routed to the photodetectors. Plot taken from $[89] . \ldots \ldots \ldots \ldots$

2.6 Schematic drawing of the scintillator system readout for a module. Plot taken from [89]. . . . . . . . . . . . . . . . .

2.7 Layout of far detector scintillator modules. The center four modules contain twenty strips and the outer modules contain 28 strips. . . . . 68 
2.8 Schematic overview of the MINOS far detector readout electronics. Three PMTs are connected with short flat ribbon cables to the VFB, which also houses two PIN diodes to monitor the light level of the light injection system. The VA ASIC amplifies and holds the PMT signals, which are multiplexed via an analog link onto an ADC on the VARC. The VFB is controlled through a digital link by the VARC. Plot taken

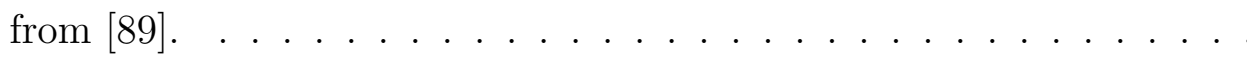

2.9 The four different configurations of planes used in the Near Detector, showing the different layouts of the scintillator modules. The upper two figures show partially instrumented planes ("calorimeter region") while the lower two figures show the fully instrumented ones ("tracking region"). The G-N notations denote the different shapes of the scintillator modules. The beam is centered midway between the coil hole and the left side of the plane, hence the scintillator need only cover that area in the target region. Plot taken from [89]. . . . . . . . .

2.10 Four logical sections of the Near Detector. The partially instrumented region only has scintillator in every 5th plane. (Plot courtesy Debdatta

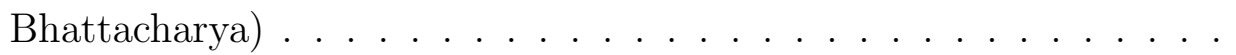

2.11 Calibration scheme for data. The main branch is the energy branch which converts raw ADC's into calibrated Muon Energy Units (MEU). The secondary branch converts ADC to photoelectrons. Plot taken from $[92] . \ldots \ldots \ldots \ldots \ldots \ldots \ldots \ldots \ldots \ldots \ldots \ldots \ldots \ldots$

2.12 Detector response to cosmic ray muons as a function of distance from strip center for a typical strip in the Near Detector. (Plot courtesy Jiajie Ling) . . . . . . . . . . . . . . . . . . . . . . . . . 80 
2.13 MINOS calorimetric response to pions and electrons at three momenta. The calorimeter-signal scale is in arbitrary units. The data (open symbols), obtained from the calibration detector exposure to CERN test beams, are compared to distributions from Monte Carlo simulations. Pion induced showers are simulated using the GCALOR shower code. Plot taken from $[89] . \ldots \ldots \ldots$. . . . . . . . . . . . 81

3.1 Probability of $\nu_{\mu} \rightarrow \nu_{e}$ conversion from FNAL to Soudan as a function of neutrino energy for different $\delta_{C P}$ values and for the normal neutrino mass hierarchy (top) and the inverted mass hierarchy (bottom). . . . 90

3.2 Probability of $\nu_{\mu} \rightarrow \nu_{e}$ conversion from FNAL to Soudan as a function of neutrino energy for different $\delta_{C P}$ values. Results are shown without the matter effects (solid) and with matter effects included (dashed and dotted). The dashed line (the upper line) assumes a normal mass hierarchy while the dotted line (the lower line) assumes a inverted mass hierarchy. . . . . . . . . . . . . . . . .

4.1 A typical $2 \mathrm{GeV} /$ c electron (top 2 plots) and a typical $2 \mathrm{GeV} /$ c pion (bottom 2 plots) in two views in the CalDet. The color scale represents the pulse-height which is in MIPs. . . . . . . . . . . .

4.2 The average longitudinal shower profile for $2 \mathrm{GeV} / \mathrm{c}$ electrons and pions in CalDet. The error bar represents the spread (rms) of energy loss. . 100

4.3 The average longitudinal shower profile for electrons in CalDet with various momenta. . . . . . . . . . . . . . . . . . . . . . . 102

4.4 Best fit parameters $E_{0}, a$ and $b$ as a function of electron energy. . . . 103

4.5 Maximum shower development $t_{\max }$ as a function of $y=E / E_{c}$. . . 104 
4.6 Fraction of energy loss as a function of the transverse position for 2 $\mathrm{GeV} / \mathrm{c}$ electrons and pions in CalDet. The error bar represents the spread (rms) of the fractional energy loss. . . . . . . . . . . . . . 105

4.7 Fraction of energy loss as a function of the transverse position for electrons in CalDet with various momenta. . . . . . . . . . . . . 106

4.8 Average shower width as a function of the electron energy. . . . . . . 107

4.9 FD Track planes and track-like planes distributions after fiducial volume cuts.

4.10 FD Reconstructed energy distributions after fiducial volume cuts and track length cuts.

4.11 Distributions of the 11 variables used in the artificial neural network. Distributions are area normalized and only events passing the preselection cuts are used in making these plots. . . . . . . . . . . . . . . 112

4.12 A typical architecture of an ANN. It is composed of an input layer, a hidden layer and an output layer. . . . . . . . . . . . . . . . 116

4.13 The ANN output for signal and different background components. The signal distribution is scaled by a factor of 10 for clarity. Pre-selections cuts are applied. . . . . . . . . . . . . . . .

4.14 Figure of Merit (FOM) as a function of the cut on the ANN output. The signal is calculated at the CHOOZ limit. . . . . . . . . . . . . . 118

4.15 Number of accepted signal events and background events as a function of the cut on the ANN output. The signal is calculated at the CHOOZ limit. . . . . . . . . . . . . . . . . . . 118

4.16 Far Detector background composition after applying all the $\nu_{e}$ selection

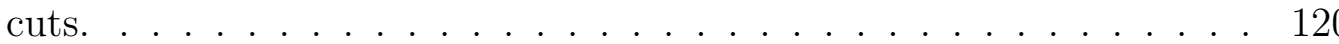

4.17 Reconstructed energy distributions of events that are accepted by the ANN as $\nu_{e}$-like events. . . . . . . . . . . . . . . . . . . . . . . 121 
4.18 Energy spectra of background events at various $\nu_{e}$ selection cut levels. 121

$4.19 \nu_{e}$ Energy spectra of signal events at various $\nu_{e}$ selection cut levels. $\quad 122$

$4.20 \nu_{e}$ selection efficiency and purity. . . . . . . . . . . . . . . 122

4.21 Breakdown of Far Detector accepted signal and background events as number of $\pi^{0}$ s in the final state. . . . . . . . . . . . . . . . . . . 123

4.22 Breakdown of Far Detector accepted signal and background events as the interaction type. . . . . . . . . . . . . . . . . 123

4.23 ND Track planes and track-like planes distributions after fiducial volume cuts. . . . . . . . . . . . . . . . . . 125

4.24 ND Reconstructed energy distributions after fiducial volume cuts and track length cuts. . . . . . . . . . . . . . . . . . 125

4.25 Distributions of the 11 variables used in the artificial neural network. Both data and $\mathrm{MC}$ are normalized to $1 \times 10^{19}$ POTs and only events passing the pre-selection cuts are used in making these plots. . . . . .

4.26 The ANN output for signal and different background components. Preselections cuts are applied. . . . . . . . . . . . . . .

4.27 Reconstructed energy distributions of events that are accepted by the ANN as $\nu_{e}$-like events. . . . . . . . . . . . . . . . . . . 128

4.28 Effects of changing hadron intranuclear rescattering on the background rates (a) ND $\left(1 \times 10^{19} \mathrm{POTs}\right)$ and (b) FD $\left(3.25 \times 10^{20} \mathrm{POTs}\right)$. The solid lines represent the $\mathrm{MC}$ with the default intranuke model while the dashed lines represent the MC with the improved intranuke model. The nominal oscillation probabilities are applied to the FD spectra. .

4.29 Far/Near ratios as a function of reconstructed energy for NC events and $\nu_{\mu} \mathrm{CC}$ events that are accepted as $\nu_{e}$ candidates. The nominal oscillation probabilities are applied to the FD spectra. ND MC and FD MC are normalized to the same exposure. . . . . . . . . . . . 131 
5.1 Simulated invariant mass distribution of inelastic events in the MINOS Near Detector using the NuMI muon neutrino beam. The shaded area shows the resonance contribution for which a different hadronization model (Rein-Sehgal) is employed. . . . . . . . . . . . . . . 136

5.2 KNO scaling distributions for $\nu p$ (left) and $\nu n$ interactions. The curve represents a fit to the Levy function. Data points are taken from [128]. 139

5.3 Nucleon $p_{T}^{2}$ distribution data from Derrick et al. [121] (left) and nucleon $x_{F}$ distribution data from Cooper et al. [122] (right). The solid lines show the AGKY parametrization, while the shaded area is the error envelope for the $1 \sigma$ variation on its free parameters. . . . . . . . . . . 140

5.4 Average charged-hadron multiplicity $\left\langle n_{c h}\right\rangle$ as a function of $W^{2}$. (a) $\nu p$ events. (b) $\nu n$ events. Data points are taken from $[128,130] . . .148$

5.5 (a) The dispersion $D_{-}=\left(\left\langle n_{-}^{2}\right\rangle-\left\langle n_{-}\right\rangle^{2}\right)^{1 / 2}$ as a function of $\left\langle n_{-}\right\rangle$. (b) $D /\left\langle n_{c h}\right\rangle$ as a function of $W^{2}$. Data points are taken from [128]. . . . 148

5.6 (a) Average multiplicity of $\pi^{0}$ mesons as a function of $W^{2}$. (b) Dispersion of the distributions in multiplicity as a function of the average multiplicity of $\pi^{0}$ mesons. Data points are taken from [119, 131, 132]

5.7 Average $\pi^{0}$ multiplicity $\left\langle n_{\pi^{0}}\right\rangle$ as a function of the number of negative hadrons $n_{-}$for different intervals of $W$. Data points are taken from $[132]$

5.8 Average charged-hadron multiplicity in the forward and backward hemispheres as functions of $W^{2}$ : (a) $\nu p$, forward, (b) $\nu p$, backward, (c) $\nu n$, forward, (d) $\nu n$, backward. Data points are taken from [128, 132, 134]. 151

5.9 Fragmentation functions for positive (a) and negative (b) hadrons. Applied cuts: $W^{2}>5\left(\mathrm{GeV} / \mathrm{c}^{2}\right)^{2}, Q^{2}>1(\mathrm{GeV} / \mathrm{c})^{2}$. Data points are taken from $[134] \ldots \ldots \ldots \ldots$ 
5.10 Mean value of the transverse momentum of charged hadrons as a function of $W$ for the selections (a) $x_{F}>0$, (b) $x_{F}<0$, and (c) all $x_{F}$.

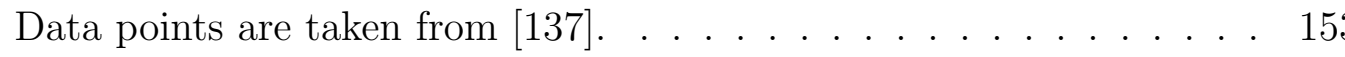

5.11 Mean value of the transverse momentum of charged hadrons as a function of $x_{F}$ for $\bar{\nu} p$. (a) $W<4 \mathrm{GeV} / c^{2}$, (b) $W>4 \mathrm{GeV} / c^{2}$. Data points are taken from $[137] . \ldots \ldots \ldots \ldots$

6.1 Near Detector strip pulse-height distributions (measured in photoelectrons - PEs) of events passing the Near Detector fiducial volume cuts (left) and passing the $\nu_{e}$ pre-selection cuts (right). Different components of MC simulation are also shown. Data and MC are sampled from March 2005 to June 2007 to average the temporal variation of PMT gains. . . . . . . . . . . . . . . . . .

6.2 Event displays of one Near Detector MC event. (a) shows the standard reconstructed event; (b) shows the same event after removing low pulseheight hits $(<2 \mathrm{PE})$ at the reconstruction level. The cross represents the reconstructed event vertex. The color scale represents the strip pulseheight which is in MIPs. . . . . . . . . . . . . . . .

6.3 Reconstructed quantities before (black) and after (red) applying the 2PE cut (ND MC). A track cut track length < 25 planes was applied in figures (b) and (c) to only accept events without a long muon

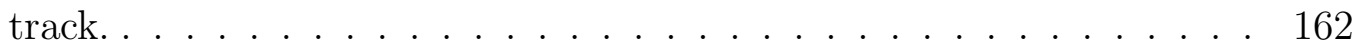

$6.4 \nu_{e}$ analysis variables before (black) and after (red) applying the $2 \mathrm{PE}$ cut (ND MC). $\nu_{e}$ pre-selection cuts are applied. . . . . . . . . . . . 163 
6.5 The M16 PMT mounting assembly. 8 fibers are placed on each M16 pixel. The Near Detector M64 mounting assembly is identical except that only one fiber is placed on each pixel. The fiber "cookie" layouts used in the two cases are shown face-on in the lower right of the figure. 164

6.6 The mappings of plane strip number in the M64 PMT pixels (left) and M16 PMT pixels (right). The former serves one ND partial U plane and the latter servers $2 / 3$ of one FD plane. . . . . . . . . . . 166

6.7 Two examples of cosmic ray events: (a) ND and (b) FD. The black boxes represent the track hits while the blue/purple hits around the track are crosstalk hits. The strip pulse-height is plotted in MIPs. . . 167

6.8 Cosmic ray muon zenith angle and azimuth angle distributions: (a) ND and (b) FD. . . . . . . . . . . . . . . . . . 169

6.9 ND crosstalk distributions: strip pulse-height (left), relative position (middle), and relative time (right)

6.10 FD crosstalk distributions: strip pulse-height (left), relative position (middle), and relative time (right)

6.11 ND crosstalk distributions: strip pulse-height (left) and relative position (right). The red lines represent the default $\mathrm{MC}$ while the blue lines represent the modified $\mathrm{MC}$ where the crosstalk fraction is scaled up by $35 \%$.

6.12 FD crosstalk distributions: strip pulse-height (left) and relative position (right). The red lines represent the default $\mathrm{MC}$ while the blue lines represent the modified $\mathrm{MC}$ where the diagonal crosstalk fraction is scaled up by $40 \%$ while the other crosstalk is scaled down by $5 \%$.

6.13 Strip pulse-height of the muon hits read out by Pixel 36 of M64 PMT (middle plot) and the crosstalk generated by Pixel 36 (surrounding plots). . . . . . . . . . . . . . . . . . . . 177 
6.14 Crosstalk charge as a function of injected charge (injected pixel is Pixel

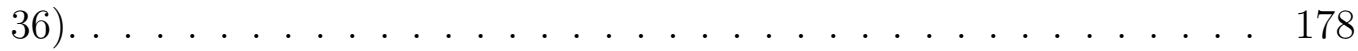

6.15 Fitted crosstalk charge as a function of injected charge for Pixel 36 in the adjacent and diagonal neighbors. Hits whose pulse-heights are greater than 0.8 PE are taken as optical crosstalk (a) while those whose pulse-heights are less than 0.5 PE are taken as electrical crosstalk (b). To get the correct mean of the crosstalk charge, zero charge hits are included in both samples. . . . . . . . . . . . . . . . . . . . 180

6.16 Measured crosstalk magnitude for ND M64 PMT pixels: (a) overall crosstalk for each of the 64 pixels, (b) optical adjacent crosstalk for each pixel, and (c) optical diagonal crosstalk for each pixel. . . . . . .

6.17 The correction factors we need to apply to the ND MC simulation: (a) optical adjacent crosstalk, (b) optical diagonal crosstalk, (c) electrical adjacent crosstalk, and (d) electrical diagonal crosstalk. . . . . . . .

6.18 Measured crosstalk magnitude for FD M16 PMT spots: (a) overall crosstalk for each of the 128 spots, (b) optical adjacent crosstalk for each spot, and (c) optical diagonal crosstalk for each spot. . . . . . . 182

6.19 The position of Pixel 5 of the 8 spots corresponding to the location of the 8 fibers coupled to that pixel. . . . . . . . . . . . . . . . . 182

6.20 The correction factors we need to apply to the FD MC simulation: (a) optical adjacent crosstalk, (b) optical diagonal crosstalk, (c) electrical adjacent crosstalk, and (d) electrical diagonal crosstalk. . . . . . . . .

6.21 ND crosstalk distributions: strip pulse-height (left) and relative position (right). The red lines represent the default $\mathrm{MC}$ while the blue lines represent the MC with updated crosstalk fractions. . . . . . . . 184 
6.22 FD crosstalk distributions: strip pulse-height (left) and relative position (right). The red lines represent the default MC while the blue lines represent the $\mathrm{MC}$ with updated crosstalk fractions. . . . . . .

6.23 Energy spectra of background events accepted by the new ANN PID as $\nu_{e}$ candidates. (a) ND (b) FD. The solid lines represent MC with the older crosstalk fractions (Def) while the dashed lines represent MC with the new crosstalk fractions (NXT). The contribution of $\nu_{\tau}$ events is not included in the FD spectra. The oscillation parameters are $\left|\Delta m_{31}^{2}\right|=2.4 \times 10^{-3} \mathrm{eV}^{2}, \sin ^{2} 2 \theta_{13}=0.15, \sin ^{2} 2 \theta_{23}=1$. The spectra are normalized to FD POT $=3.25 \times 10^{20}$ (2-year exposure) and ND $\mathrm{POT}=1 \times 10^{19}$. The errors are only statistical errors. . . . . . 187

7.1 Breakdown of ND neutrino spectrum in the low energy (LE) beam configuration. Diagram shows the 5 general trajectories of mesons that pass through the horns. (Plot courtesy Zarko Pavlovic) . . . . . 192

7.2 The simulated $\nu_{\mu} \mathrm{CC}$ event rate as a function of $E_{\nu}$ in the ND with two different horn current configurations: black-185kA, red-0kA. . .

7.3 The reconstructed energy spectra of events that pass the $\nu_{e}$ selection algorithm. Left: horn-on; Right: horn-off. All spectra are normalized to $1 \times 10^{19}$ POTs. . . . . . . . . . . . . . . . . . . . . .

7.4 The true energy spectra of ND beam $\nu_{e}$ events. Left: events passing fiducial volume cuts; Right: events passing all $\nu_{e}$ selection cuts. All spectra are normalized to $1 \times 10^{19}$ POTs. . . . . . . . . . . . . . . 201

7.5 The ND horn-off over horn-on spectrum ratios. The error bars represent the MC statistics. . . . . . . . . . . . . . . . . . . . 202 
7.6 $r_{N C}$ in bins of reconstructed energy. (a) After fiducial volume cuts. The black points are from NOT- $\nu_{\mu} \mathrm{CC}(\mathrm{NC})$ selected data events. The red points are from NOT- $\nu_{\mu}$ CC (NC) selected Monte Carlo events. (b) From true $\mathrm{NC}$ events. The black points are after the fiducial volume cuts. The blue points are after the pre-selection cuts. The red points are after the ANN PID cut. The error bars represent the MC statistics. 203

7.7 (a) Average $W^{2}$ as a function of true shower energy for events in the horn-on (black) and horn-off (red) configurations. (b) Average shower angle as a function of true shower energy for events in the horn-on (black) and horn-off (red) configurations. Fiducial volume cuts are applied. . . . . . . . . . . . . . . . . . . . . 204

7.8 Selection efficiency for ANN algorithm as a function of the shower angle with respect to the beam for events with true shower energy between 3 and $4 \mathrm{GeV} . \ldots \ldots \ldots \ldots \ldots \ldots$

7.9 Impact of flux uncertainties in the horn off/on ratios. The error bars represent the MC statistical error. . . . . . . . . . . . . . 206

7.10 Impact of cross section uncertainties in the horn off/on ratios. The error bars represent the MC statistical error. . . . . . . . . . . . . 207

7.11 Impact of hadronization model uncertainties on the horn off/on ratios. The error bars represent the MC statistical error. . . . . . . . . . 209

7.12 Impact of intranuke rescattering uncertainties on the horn off/on ratios. The error bars represent the MC statistical error. . . . . . . . . . 210

7.13 Impact of crosstalk mismodeling on the horn off/on ratios. The error bars represent the MC statistical error. . . . . . . . . . . . . . 210 
7.14 The reconstructed energy spectra of events that pass the $\nu_{e}$ selection algorithm. Left: horn-on; Right: horn-off. All spectra are normalized to $1 \times 10^{19}$ POTs. The error bar represents the statistical error while the error band represents the systematic error. . . . . . . . . . . . . . 213

7.15 The ND horn-off over horn-on spectrum ratios. The error bars represent the MC statistics. The error bar represents the statistical error while the error band represents the systematic error. . . . . . . . . . 214

7.16 Reconstructed horn-on energy spectrum of events that pass the $\nu_{e}$ selection algorithm assuming $15 \%$ beam $\nu_{e}$ error. . . . . . . . . . . . 215

8.1 Extrapolation of $\mathrm{NC}$ and $\nu_{\mu} \mathrm{CC}$ background components from ND to FD taking into account oscillations. The ND spectra are obtained using the horn on/off background decomposition method. ND spectra are normalized to $1 \times 10^{19}$ POTs and FD spectra are normalized to $3.25 \times 10^{20}$ POTs. . . . . . . . . . . . . . . . . 220

8.2 Predicted FD background energy spectra for an exposure of $3.25 \times 10^{20}$

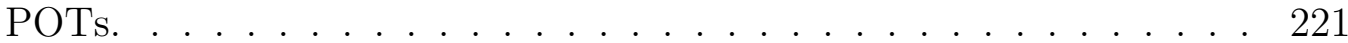

8.3 Comparison of PID distributions for the first event in each spill (black points) and for all events (red curve). Distributions are area normalized. (a) Data; (b) MC; (c) Double ratio. . . . . . . . . . . . . . . 228

8.4 ANN PID distributions for MC with perfect geometry and with real geometry.

8.5 $\chi^{2}$ distributions as a function of $\sin ^{2}\left(2 \theta_{13}\right)$ and $\delta_{C P}$. We assume $\left|\Delta m_{32}^{2}\right|=$ $0.0024 e V^{2}$ and $\sin ^{2} 2 \theta_{23}=1$. The exposure is taken to be $3.25 \times 10^{20}$ POTs. Left: normal mass hierarchy $\left(\Delta m_{32}^{2}>0\right)$; Right: inverted mass hierarchy $\left(\Delta m_{32}^{2}<0\right)$. . . . . . . . . . . . . . . . . 239 
$8.6 \chi^{2}$ distributions as a function of $\sin ^{2}\left(2 \theta_{13}\right)$. We assume $\delta_{C P}=0$, $\left|\Delta m_{32}^{2}\right|=0.0024 \mathrm{eV}^{2}$ and $\sin ^{2} 2 \theta_{23}=1$. The exposure is taken to be $3.25 \times 10^{20}$ POTs. . . . . . . . . . . . . . . . . . . . . . . 239

8.7 MINOS sensitivity as a function of $\delta_{C P}$ at $3.25 \times 10^{20}$ POTs. . . . . . 240

9.1 Reconstructed vertex distributions of neutrino events in the Far Detector fiducial volume. . . . . . . . . . . . . . . . . 245

9.2 Reconstructed track quantities after fiducial volume cuts. . . . . . . . 246

9.3 Reconstructed shower quantities after fiducial volume cuts. . . . . . . 246

9.4 Reconstructed $Q^{2}$ and $W^{2}$ after fiducial volume cuts. . . . . . . . . . 247

9.5 Distributions of the 11 variables used in the ANN after pre-selection cuts. The last plot shows the $\chi^{2} / \mathrm{NDF}$ of the 11 distributions. No ND data based correction was applied to MC. . . . . . . . . . . . . 250

9.6 ANN PID distributions for data (black points), corrected background only MC (red) and the raw background only MC (blue). The bin below zero corresponds in an underflow bin which contains events with no assigned PID. The correction to MC takes into account the data/MC differences in the ND PID distributions. . . . . . . . . . . . . . . 251

9.7 ANN PID distributions for data (black points), corrected background only MC (red) and background+signal MC (blue). Signal is computed at $\sin ^{2}\left(2 \theta_{13}\right)=0.15$ (CHOOZ limit). The bin below zero corresponds in an underflow bin which contains events with no assigned PID. . .

9.8 Distributions of events accepted by ANN (a) Vertex z; (b) Vertex x vs y; (c) Events per unit POT vs time (each bin corresponds to 2 month of data). . . . . . . . . . . . . . . . 253 
9.9 Reconstructed energy of selected data compared to predictions in the signal region. Signal is computed at $\sin ^{2}\left(2 \theta_{13}\right)=0.15$ (CHOOZ limit). Plots are stack plots. . . . . . . . . . . . . . . . . 254

$9.10 \chi^{2}$ distributions as a function of $\sin ^{2}\left(2 \theta_{13}\right)$ and $\delta_{C P}$ for observation of 35 data events. Left: normal mass hierarchy $\left(\Delta m_{32}^{2}>0\right)$; Right: inverted mass hierarchy $\left(\Delta m_{32}^{2}<0\right)$. . . . . . . . . . . . . . 254

$9.11 \chi^{2}$ distributions as a function of $\sin ^{2}\left(2 \theta_{13}\right)$ for observation of data 35 events. We assume $\delta_{C P}=0 \ldots \ldots \ldots$. . . . . . . . . . 255

9.12 Best fit and $90 \%$ upper limit in $\sin ^{2} 2 \theta_{13}$ as a function of $\delta_{C P}$ for the normal mass hierarchy (red) and the inverted mass hierarchy (blue) at the MINOS best fit value for $\Delta m_{32}^{2}$ and $\sin ^{2} 2 \theta_{23}$ for $3.14 \times 10^{20}$ POTs. 256 
xxxii 


\section{Chapter 1}

\section{Introduction}

\subsection{A Brief History of Neutrinos}

The history of neutrino physics began with Wolfgang Pauli's often quoted letter to the Physical Society of Tübingen on the 14th of December 1930 [1], in which he postulates the existence of a new particle, which he called the neutron, in order to explain the observed continuous electron spectrum accompanying nuclear beta decay. Pauli required his hypothetical particle to be electrically neutral and have spin $1 / 2$, to ensure conservation of electric charge and angular momentum. It can only interact weakly and its rest mass was expected to be small, but not necessarily vanishing. In 1932 Chadwick discovered what we now call the neutron [2], but it was clear that this neutral particle was too heavy to be the neutron that Pauli had predicted and hence later on Pauli's neutron was renamed the neutrino by Enrico Fermi. In 1934 Fermi proposed his famous theory of weak interaction [3], based on which Bethe \& Peierls predicted the cross section for the interaction of the neutrino with matter to be extremely small [4].

By 1950, there was compelling theoretical evidence for the existence of neutrinos, but there was still no direct experimental verification. In the mid-fifties, the decisive 
experiments were conducted at the Savannah River nuclear reactor in South Carolina. Here Frederick Reines and Clyde Cowan, both from Los Alamos Laboratory, set up a large tank of water and watched for the "inverse" beta-decay reaction

$$
\bar{\nu}_{e}+p^{+} \rightarrow n+e^{+}
$$

At their detector the antineutrino flux was calculated to be $5 \times 10^{13}$ particles per square centimeter per second, but even at this fantastic intensity they could only hope for two or three events every hour. On the other hand, they developed an ingenious delayed-coincidence technique, which made use of the positron to produce the first pulse and the $\gamma$ 's from the captured neutron for the second pulse. After months of data collection, they had accumulated data on about three neutrinos per hour in their detector. The signal to background ratio was about three to one. Their results provided unambiguous confirmation of the neutrino's existence [5]. Frederick Reines was awarded the Nobel prize in 1995 for this work.

In 1958, Maurice Goldhaber, Lee Grodzins, and Andrew Sunyar at Brookhaven National Laboratory demonstrated that the new neutrino has left-handed (negative) helicity by a combined analysis of circular polarization and resonant scattering of $\gamma$ rays following orbital electron capture ${ }^{1}[7]$. This result is in agreement with the $\mathrm{V}-\mathrm{A}$ nature of the weak interaction predicted by Feynman and Gell-Mann [8].

In 1962, the muon-neutrino was identified in an experiment at the Brookhaven AGS [9], and this marked the beginning of the fruitful use of high-energy neutrino beams at accelerators. In 1987, Jack Steinberger, Leon Lederman, and Mel Schwartz won the Nobel Prize for this discovery. In 1975, a new lepton, the tau, was discovered by a group led by physicist Martin Perl at the Stanford Linear Accelerator Center

\footnotetext{
${ }^{1}$ Electron capture is one form of radioactivity. A parent nucleus may capture one of its orbital electrons and emit a neutrino. This is a process that competes with positron emission and has the same effect on the atomic number. Most commonly, it is a K-shell electron that is captured, and this is referred to as K-capture. A typical example is ${ }_{4}^{7} \mathrm{Be}+{ }_{-1}^{0} e \rightarrow{ }_{3}^{7} \mathrm{Li}+\nu$.
} 
[10]. Experiments performed shortly afterward provided strong evidence that there also exists a third species of neutrino, the tau neutrino, $\nu_{\tau}$. In 1995, Perl and Reines won the Nobel Prize for their discoveries. The tau neutrino was first detected in 2000 by the DONUT experiment [11].

In 1973, the Gargamelle experiment at CERN discovered the weak neutral current $(\mathrm{NC})$ interaction

$$
\bar{\nu}_{\mu}+e \rightarrow \bar{\nu}_{\mu}+e
$$

mediated by the $Z^{0}$ boson. The same series of experiments also showed evidence of the corresponding neutrino-quark process by observing events induced by $\nu / \bar{\nu}$ that produced hadrons, but no muon or electron [12]:

$$
\begin{gathered}
\bar{\nu}_{\mu}+N \rightarrow \bar{\nu}_{\mu}+X \\
\nu_{\mu}+N \rightarrow \nu_{\mu}+X
\end{gathered}
$$

Observation of the neutral current interaction lent strong support to a unified theory of weak and electromagnetic interactions proposed a few years earlier by Sheldon Glashow [13], Abdus Salam [14], and Steven Weinberg [15], which became part of the more encompassing framework of the "Standard Model". This model is capable of describing all the known physics of weak and electromagnetic interactions, incorporating all the experimental results at energies available at present accelerators. The observation of the neutral weak current and the discovery of the intermediate vector bosons $W[16]$ and $Z$ [17] at CERN in 1983, at $M_{W}=82 \mathrm{GeV} / c^{2}$ and $M_{Z}=92$ $\mathrm{GeV} / c^{2}$ (as predicted), contributed spectacularly to the success of this model.

Two neutrino related major discoveries were made in the 1980s. Firstly neutrinos from outside our solar system were observed for the first time, when observations of neutrino events were correlated with astronomical observations of the supernova SN 1987A [27]. On earth 19 low-energy anti-neutrino events were observed in two large 
water Cerenkov detectors (Kamiokande II and IMB) within about 10 seconds. Many determinations of neutrino properties including limits on neutrino mass, lifetime and magnetic moment were extracted from the supernova data.

Secondly the Mark II experiment at SLC and the LEP experiments at CERN precisely measured the width of the $Z$ resonance. Those measurements were highly significant for neutrino physics as it provided very strong evidence there were only 3 light $\left(m_{\nu}<45 \mathrm{GeV} / c^{2}\right)$, active neutrino flavors [28, 29].

The rest of this section is devoted to the discussions of the phenomenon "neutrino oscillations" whereby a neutrino created with a specific lepton flavor (electron, muon or tau) can later be measured to have a different flavor. Neutrino oscillations are of theoretical and experimental interest as observation of the phenomenon implies that the neutrino has a non-zero mass, which is not part of the original Standard Model of particle physics.

In 1957, an Italian physicist, Bruno Pontecorvo, living in the USSR, discussed the possibility of neutrino-antineutrino oscillations [6]. This was the first mention of "neutrino oscillations", albeit not of the kind that have become familiar in recent years.

In 1959, Ray Davis et al. showed that the anti-neutrino could be distinguished from the neutrino while at the same time putting an upper limit on the neutrino flux from the sun [18]. In the late 1960s, Ray Davis's Homestake Experiment was the first to measure the flux of neutrinos from the sun and detect a deficit compared with the prediction of John N. Bahcall's Standard Solar Model [19] and the discrepancy essentially created the solar neutrino problem. The experiment used a chlorine-based detector. Many subsequent experiments confirmed the deficit, including Kamiokande and Super-Kamiokande in Japan and SNO (Sudbury Neutrino Observatory) in Ontario, Canada. The first scientific results of SNO were published in 2001 [20], bringing the first clear evidence that neutrinos change flavor, or oscillate, as they travel through 
the sun, which resolved the solar neutrino problem. In 2002, Ray Davis and Masatoshi Koshiba won the Nobel Prize for the detection of solar neutrinos.

The neutrinos produced in the earth's atmosphere as the result of the decay of cosmic-ray induced pions and kaons were first detected by experiments performed in the early 1960s. These experiments were located in the Kolar Gold Field in Southern India [21] and at the East Rand Proprietary mine in South Africa [22]. In the 1980s several large water Ĉerenkov detectors were being used to search for proton decay. The major source of background to these proton decay experiments was from neutrinos created in the atmosphere by high energy cosmic rays. When proton decay wasn't discovered, ironically it was the background that brought fascinating new physics. The American experiment, IMB [24], and the Japanese experiments, Kamiokande [25] and Super-K [50], reported a deficit in the number of muon neutrinos created in the atmosphere with respect to the number of electron neutrinos. This deficit became known as the atmospheric neutrino anomaly and it provided the evidence for neutrino oscillations. Since the atmosphere is not a very well controlled source of neutrinos a confirmation that the atmospheric results were due to the oscillations was sought. The K2K experiment [26] in Japan provided that reassurance. They generated a very pure beam of muon neutrinos with the accelerator at the KEK lab directed toward the SuperK detector $\sim 250 \mathrm{~km}$ away. They were able to demonstrate the disappearance of the muon neutrinos consistent with the predictions with the parameters inferred from the atmospheric measurements.

With the K2K experiment confirming the disappearance of muon neutrinos as a result of oscillations it falls to the MINOS experiment to make a precision measurement of the effect and further constrain the atmospheric oscillation parameters. The MINOS experiment also has the potential to further constrain or make the first measurement of the still unmeasured neutrino mixing angle $\theta_{13}$. The measurement of $\theta_{13}$ in the MINOS experiment forms the focus of this thesis. More details on the 
neutrino oscillation experiments are further discussed later in this chapter.

\subsection{The Standard Electro-Weak Interaction Model}

\subsubsection{Gauge Theories}

The Standard Model is a collection of related theories, incorporating quantum electrodynamics, the Glashow-Weinberg-Salam theory of electroweak processes, and quantum chromodynamics. It describes all of the known elementary particle interactions except gravity. The Standard Model has met almost every experimental test. It has, moreover, an attractive aesthetic feature: in the Standard Model all of the fundamental interactions derive from a single general principle, the requirement of local gauge invariance. Yet, despite its success, the Standard Model appears in need of extension and generalization. In its present form it is not capable of predicting the masses of the fermions, nor can it explain why there are several fermion families. The study of neutrino physics can potentially lead to the discovery of new physics beyond the Standard Model, and this is the chief reason why the neutrino is such an interesting particle. In the Standard Model neutrinos are massless particles; however, there is compelling experimental evidence that neutrino flavor oscillations do occur both in the vacuum and in matter, which is a strong indication of the existence of neutrino mass. As such, massive neutrinos are the only experimentally verified occurrence of physics beyond the Standard Model at the present time.

In the Standard Model, there exist three kinds of elementary particles: leptons, quarks and mediators. There are six leptons, classified according to their charge $(Q)$, electron number $\left(L_{e}\right)$, muon number $\left(L_{\mu}\right)$, and tau number $\left(L_{\tau}\right)$. They fall naturally 
into three families (or generations):

$$
\left(\begin{array}{c}
\nu_{e} \\
e
\end{array}\right)\left(\begin{array}{c}
\nu_{\mu} \\
\mu
\end{array}\right)\left(\begin{array}{c}
\nu_{\tau} \\
\tau
\end{array}\right) .
$$

There are also six antileptons. So there are really 12 leptons.

Similarly, there are six "flavors" of quarks, which are classified according to charge, strangeness $(S)$, charm $(C)$, beauty $(B)$ and truth $(T)$. The quarks, too, fall into three generations:

$$
\left(\begin{array}{l}
u \\
d
\end{array}\right)\left(\begin{array}{l}
c \\
s
\end{array}\right)\left(\begin{array}{l}
t \\
b
\end{array}\right) .
$$

There are also six antiquarks. Meanwhile, the quarks and antiquarks possess an additional quantum number, and each quark and antiquark exist in three unitary equivalent states, differing in values of the new quantum number, termed color. Therefore there are 36 quarks and antiquarks in all.

Finally, every interaction has its mediators: the photon for the electromagnetic force, two $W$ 's and a $Z$ for the weak force, the graviton (presumably) for gravity, eight gluons for the strong force. The gluons themselves carry color, and therefore (like the quarks) should not exist as isolated particles. There are 12 mediators in all.

Particles with half-integer spin are known as fermions - all leptons and quarks are fermions; particles with integer spin are known as bosons - all mediators are bosons.

The Standard Model is an $S U(3) \otimes S U(2)_{L} \otimes U(1)_{Y}$ gauge theory. This theory is now believed to describe all elementary particle interactions except gravity. We start with $U(1)$ symmetry, which leads to the Electrodynamics theory. In Quantum Field Theory, particles are defined in terms of their Lagrangians and described by field equations. The Lagrangian for a free fermion is the Dirac Lagrangian

$$
\mathcal{L}=\bar{\psi}\left(i \gamma^{\mu} \partial_{\mu}-m\right) \psi,
$$


where $\psi$ is the field describing the dynamics of the particle and $m$ is its mass. $\gamma^{\mu}$ are the Dirac matrices. This Lagrangian is invariant under the transformation

$$
\psi \longrightarrow e^{i \alpha} \psi
$$

where $\alpha$ is any real number. We call (1.5) a global gauge transformation. The Dirac Lagrangian is not invariant under the local gauge transformation

$$
\psi \longrightarrow e^{i \alpha(x)} \psi
$$

where $\alpha$ is a function of $x^{\mu}$. It can be verified that under the local gauge transformations

$$
\mathcal{L} \longrightarrow \mathcal{L}-\left(\partial_{\mu} \alpha\right) \bar{\psi} \gamma^{\mu} \psi
$$

If we demand that the Dirac Lagrangian be invariant under local gauge transformations we have to add one additional term in order to soak up the extra term in Eq. (1.7). Suppose

$$
\mathcal{L}=\bar{\psi}\left(i \gamma^{\mu} \partial_{\mu}-m\right) \psi-e \bar{\psi} \gamma^{\mu} \psi A_{\mu}
$$

Where $A_{\mu}$ is some new field (called a "gauge" field or a "gauge" boson) that transforms under local gauge transformations according to the rule

$$
A_{\mu} \longrightarrow A_{\mu}-\frac{1}{e} \partial_{\mu} \alpha(x)
$$

The new Lagrangian is now invariant under local gauge transformations. To complete the construction of a locally invariant Lagrangian, we must find a kinetic energy term for the field $A_{\mu}$. Since it is a vector field, we look to the Proca Lagrangian which 
describes a massive spin-1 field of mass $m_{A}$

$$
\mathcal{L}=-\frac{1}{4} F^{\mu \nu} F_{\mu \nu}+\frac{1}{2} m_{A}^{2} A^{\nu} A_{\nu}
$$

$F^{\mu \nu} \equiv \partial^{\mu} A^{\nu}-\partial^{\nu} A^{\mu}$ is invariant under (1.9) but $A^{\nu} A_{\nu}$ is not. Evidently, the gauge field must be massless $\left(m_{A}=0\right)$, otherwise local gauge invariance will be lost.

The conclusion is that if we start with the Dirac Lagrangian, and impose local gauge invariance, we are forced to introduce a massless vector field $\left(A^{\mu}\right)$, and the complete Lagrangian becomes

$$
\mathcal{L}=\bar{\psi}\left(i \gamma^{\mu} \partial_{\mu}-m\right) \psi-\frac{1}{4} F^{\mu \nu} F_{\mu \nu}-e \bar{\psi} \gamma^{\mu} \psi A_{\mu}
$$

The last term $-e \bar{\psi} \gamma^{\mu} \psi A_{\mu}$ describes the coupling of fermion fields $\psi, \bar{\psi}$ to the gauge field $A^{\mu}$. We can define a new derivative, called the covariant derivative, as follows

$$
D_{\mu} \equiv \partial_{\mu}+i e A_{\mu}
$$

and the new Lagrangian becomes

$$
\begin{aligned}
\mathcal{L} & =\bar{\psi}\left(i \gamma^{\mu} D_{\mu}-m\right) \psi-\frac{1}{4} F^{\mu \nu} F_{\mu \nu} \\
& =\bar{\psi}(i \not D-m) \psi-\frac{1}{4} F^{\mu \nu} F_{\mu \nu}
\end{aligned}
$$

where $\not D \equiv \gamma^{\mu} D_{\mu}$. This is the Lagrangian for Quantum Electrodynamics (QED) and $A_{\mu}$ is the photon $(\gamma)$ field required to preserve the local gauge invariance.

The local gauge transformation (1.6) may be thought of as multiplication of $\psi$ by a unitary $1 \times 1$ matrix

$$
\psi \longrightarrow U \psi \text {, where } U^{\dagger} U=1 \text {. }
$$

(Here $U=e^{i \alpha}$ ). The group of all such matrices is $U(1)$; hence the symmetry involved 
is called " $U(1)$ gauge invariance".

The $S U(3)$ gauge invariance in the Standard Model is imposed to describe Quantum Chromodynamics (QCD), the theory of strong interactions and color is the conserved quantity. The corresponding gauge bosons are eight massless gluons.

The $S U(2)_{L} \otimes U(1)_{Y}$ symmetry leads to the Electroweak theory which unifies the electromagnetic and weak interactions. Requiring this invariance introduces four gauge fields. Three of these fields couple to the weak isospin carried by left-handed fermions and the last couples to the weak hypercharge carried by all fermions.

The principle of local gauge invariance works beautifully for the strong and electromagnetic interactions. It provides a mechanism for determining the couplings. Moreover, as 't Hooft and others proved in the early seventies [30], gauge theories are automatically renormalizable. But the application to weak interactions was stymied by the fact that gauge fields have to be massless. The mass term in the Proca Lagrangian (1.10) is not locally gauge invariant, and whereas the photon and the gluons are massless, the $W^{\prime}$ 's and the $Z^{0}$ certainly are not. Another process is required to connect the gauge fields to the $W, Z$ and $\gamma$ bosons and to generate the masses of the $W$ and $Z$ bosons. This process is called the Higgs Mechanism.

\subsubsection{Spontaneous Symmetry Breaking}

The Higgs Mechanism is built on the union of gauge invariance and spontaneous symmetry breaking. A massless gauge boson can acquire mass through spontaneous symmetry breaking. We begin with an Abelian gauge theory example by considering a complex scalar field coupled both to itself and to an electromagnetic field

$$
\mathcal{L}=\left|D_{\mu} \phi\right|^{2}-V(\phi)-\frac{1}{4}\left(F_{\mu \nu}\right)^{2}
$$


with $D_{\mu}=\partial_{\mu}+i e A_{\mu}$. This Lagrangian is invariant under the local $U(1)$ transformation

$$
\phi(x) \longrightarrow e^{i \alpha(x)} \phi(x), A_{\mu}(x) \longrightarrow A_{\mu}(x)-\frac{1}{e} \partial_{\mu} \alpha(x) .
$$

If we choose the potential in $\mathcal{L}$ to be of the form

$$
V(\phi)=-\mu^{2} \phi^{*} \phi+\frac{\lambda}{2}\left(\phi^{*} \phi\right)^{2},
$$

with $\mu^{2}>0$, the field $\phi$ will acquire a vacuum expectation value and the $U(1)$ global symmetry will be spontaneously broken. The minimum of this potential occurs at

$$
\langle\phi\rangle=\phi_{0}=\left(\frac{\mu^{2}}{\lambda}\right)^{1 / 2}
$$

We can expand the Lagrangian (1.15) about the vacuum state (1.18). Decompose the complex field $\phi(x)$ as

$$
\phi(x)=\phi_{0}+\frac{1}{\sqrt{2}}\left(\phi_{1}(x)+i \phi_{2}(x)\right) .
$$

The potential (1.17) is rewritten as

$$
V(\phi)=-\frac{1}{2 \lambda} \mu^{4}+\frac{1}{2} \cdot 2 \mu^{2} \phi_{1}^{2}+\mathcal{O}\left(\phi_{i}^{3}\right)
$$

The kinetic energy term of $\phi$ is rewritten as

$$
\left|D_{\mu} \phi\right|^{2}=\frac{1}{2}\left(\partial_{\mu} \phi_{1}\right)^{2}+\frac{1}{2}\left(\partial_{\mu} \phi_{2}\right)^{2}+\sqrt{2} e \phi_{0} \cdot A_{\mu} \partial^{\mu} \phi_{2}+e^{2} \phi_{0}^{2} A_{\mu} A^{\mu}+\cdots
$$

where we have omitted terms cubic and quartic in the fields $A_{\mu}, \phi_{1}$, and $\phi_{2}$. This Lagrangian describes a massive scalar field $\phi_{1}$ of mass $\sqrt{2} \mu$ and a massless scalar field $\phi_{2}$. The appearance of massless particles when a continuous symmetry is spontaneously broken is a general result, known as Goldstone's theorem. The massless fields that 
arise through spontaneous symmetry breaking are called Goldstone bosons. Many light bosons seen in physics, such as the pions, may be interpreted (at least approximately) as Goldstone bosons.

The last term written explicitly in (1.21) is a photon mass term

$$
\Delta \mathcal{L}=\frac{1}{2} m_{A}^{2} A_{\mu} A^{\mu}
$$

where the mass

$$
m_{A}^{2}=2 e^{2} \phi_{0}^{2}
$$

arises from the nonvanishing vacuum expectation value of $\phi$.

We have demonstrated how a massless gauge boson acquires a mass through spontaneous symmetry breaking. This mechanism, by which spontaneous symmetry breaking generates a mass for a gauge boson, was explored and generalized to the non-Abelian case by Higgs, Kibble, Guralnik, Hagen, Brout, and Englert, and is now known as the Higgs mechanism. We now use this mechanism to build a model that gives the experimentally correct description of the weak interactions. We start with an $S U(2)_{L} \otimes U(1)_{Y}$ gauge invariant Lagrangian, spontaneously broken by the Higgs mechanism in such a way the the final Lagrangian is invariant under the $U(1)_{e . m}$. gauge transformation.

As of now no right-handed neutrinos have been found. The left-handed and righthanded leptons can be put into the doublet and singlet representations of the $S U(2)$ group

$$
\begin{aligned}
& \left(\begin{array}{c}
\nu_{e} \\
e
\end{array}\right)_{L}\left(\begin{array}{c}
\nu_{\mu} \\
\mu
\end{array}\right)_{L}\left(\begin{array}{c}
\nu_{\tau} \\
\tau
\end{array}\right)_{L} . \\
& e_{R} \quad \mu_{R} \quad \tau_{R}
\end{aligned}
$$

Restricting our discussion to the first generation of the lepton sector, which consists of an electron-neutrino and electron left-handed doublet and a right-handed electron 
singlet, the Lagrangian which is invariant under $S U(2)_{L} \otimes U(1)_{Y}$ is given by

$$
\mathcal{L}_{0}=\bar{l}_{L} i \not D l_{L}+\bar{l}_{R} i \not D l_{R}-\frac{1}{4} \overrightarrow{F^{\mu \nu}} \cdot \overrightarrow{F_{\mu \nu}}-\frac{1}{4} G^{\mu \nu} G_{\mu \nu}
$$

where

$$
\begin{aligned}
l_{L} & =\left(\begin{array}{c}
\nu_{e} \\
e
\end{array}\right)_{L}, l_{R}=e_{R}, \\
D_{\mu} & =\partial_{\mu}-i g \vec{T} \cdot \vec{A}_{\mu}-i g^{\prime} Y B_{\mu}, \\
\vec{F}_{\mu \nu} & =\partial_{\mu} \vec{A}_{\nu}-\partial_{\nu} \vec{A}_{\mu}+g\left(\vec{A}_{\mu} \times \vec{A}_{\nu}\right), \\
G_{\mu \nu} & =\partial_{\mu} B_{\nu}-\partial_{\nu} B_{\mu} .
\end{aligned}
$$

In Eq.(1.26), $\vec{T}$ is the isospin operator $(\vec{T}=\vec{\sigma} / 2), Y$ is the hypercharge operator, and $g$ and $g^{\prime}$ are, respectively, $S U(2)_{L}$ and $U(1)_{Y}$ gauge coupling constants. Furthermore, $\overrightarrow{A_{\mu}} \equiv\left(A_{\mu}^{1}, A_{\mu}^{2}, A_{\mu}^{3}\right)$ (a vector in isospin space) and $B_{\mu}$ are massless $S U(2)_{L}$ and $U(1)_{Y}$ gauge bosons, respectively. The Lagrangian in Eq.(1.25) is invariant under the local gauge transformations given by

$$
\begin{aligned}
l_{L, R} & \rightarrow U l_{L, R}, \\
\vec{T} \cdot \vec{A}_{\mu} & \rightarrow U\left[\vec{T} \cdot \vec{A}-\frac{i}{g} U^{-1}\left(\partial_{\mu} U\right)\right] U^{-1} \\
B_{\mu} & \rightarrow B_{\mu}+\partial_{\mu} \Lambda(x)
\end{aligned}
$$

where

$$
U=\exp \left\{i g \vec{T} \cdot \vec{\Lambda}(x)+i g^{\prime} Y \Lambda(x)\right\}
$$


and $\Lambda(x)$ and $\vec{\Lambda}(x)$ are gauge functions. Defining

$$
\begin{aligned}
W_{\mu}^{ \pm} & =\frac{1}{\sqrt{2}}\left(A_{\mu}^{1} \mp i A_{\mu}^{2}\right) \\
Z_{\mu}^{0} & =\frac{1}{\sqrt{g^{2}+g^{\prime 2}}}\left(g A_{\mu}^{3}-g^{\prime} B_{\mu}\right) \\
A_{\mu} & =\frac{1}{\sqrt{g^{2}+g^{\prime 2}}}\left(g^{\prime} A_{\mu}^{3}+g B_{\mu}\right)
\end{aligned}
$$

the covariant derivative can be rewritten as

$$
\begin{aligned}
D_{\mu}= & \partial_{\mu}-i g T^{a} A_{\mu}^{a}-i g^{\prime} Y B_{\mu} \\
= & \partial_{\mu}-i \frac{g}{\sqrt{2}}\left(W_{\mu}^{+} T^{+}+W_{\mu}^{-} T^{-}\right)-i \frac{1}{\sqrt{g^{2}+g^{\prime 2}}} Z_{\mu}^{0}\left(g^{2} T^{3}-g^{\prime 2} Y\right) \\
& -i \frac{g g^{\prime}}{\sqrt{g^{2}+g^{\prime 2}}} A_{\mu}\left(T^{3}+Y\right)
\end{aligned}
$$

where

$$
T^{ \pm}=\left(T^{1} \pm i T^{2}\right)=\frac{1}{2}\left(\sigma^{1} \pm i \sigma^{2}\right)=\sigma^{ \pm}
$$

We should identify the coefficient of the electromagnetic interaction as the electron charge $e$,

$$
e=\frac{g g^{\prime}}{\sqrt{g^{2}+g^{\prime 2}}}
$$

and identify the electric charge quantum number as

$$
Q=T^{3}+Y
$$

To simplify things further, we define the weak mixing angle, $\theta_{W}$, to be the angle that 
appears in the change of basis from $\left(A^{3}, B\right)$ to $\left(Z^{0}, A\right)$

$$
\left(\begin{array}{c}
Z^{0} \\
A
\end{array}\right)=\left(\begin{array}{cc}
\cos \theta_{W} & -\sin \theta_{W} \\
\sin \theta_{W} & \cos \theta_{W}
\end{array}\right)\left(\begin{array}{c}
A^{3} \\
B
\end{array}\right)
$$

that is,

$$
\cos \theta_{W}=\frac{g}{\sqrt{g^{2}+g^{\prime 2}}}, \sin \theta_{W}=\frac{g^{\prime}}{\sqrt{g^{2}+g^{\prime 2}}} .
$$

The magnitude of the electron charge can be rewritten as

$$
e=g \sin \theta_{W}=g^{\prime} \cos \theta_{W} .
$$

We can rewrite the covariant derivative (1.29) in the form

$$
D_{\mu}=\partial_{\mu}-i \frac{g}{\sqrt{2}}\left(W_{\mu}^{+} T^{+}+W_{\mu}^{-} T^{-}\right)-i \frac{g}{\cos \theta_{W}} Z_{\mu}^{0}\left(T^{3}-\sin ^{2} \theta_{W} Q\right)-i e A_{\mu} Q
$$

The covariant derivative (1.36) uniquely determines the coupling of the $W$ and $Z^{0}$ fields to fermions, once the quantum numbers of the fermion fields are specified. Note the $W$ boson couples only to left-handed helicity states of quarks and leptons. For the right-handed fields, $e_{R}, u_{R}, d_{R}, T^{3}=0$; for the left-handed fields, $T^{3}= \pm \frac{1}{2}$,

$$
\psi_{L}=\left(\begin{array}{l}
\nu \\
e
\end{array}\right)_{L}, q_{L}=\left(\begin{array}{l}
u \\
d
\end{array}\right)_{L}
$$

Once we have specified the $T^{3}$ value for each fermion field, the value of $Y$ that we must assign follows from Eq.(1.32).

The complete lepton-gauge boson coupling can be expressed in the Lagrangian

$$
\mathcal{L}_{\text {electroweak }}=\frac{g}{\sqrt{2}}\left(W_{\mu}^{+} J_{W}^{\mu+}+W_{\mu}^{-} J_{W}^{\mu-}\right)+\frac{g}{\cos \theta_{W}} Z_{\mu}^{0} J_{Z}^{\mu}+e A_{\mu} J_{E M}^{\mu}
$$


where

$$
\begin{aligned}
J_{W}^{\mu+}= & \bar{\nu}_{L} \gamma^{\mu} e_{L}+\bar{u}_{L} \gamma^{\mu} d_{L} \\
J_{W}^{\mu-}= & \bar{e}_{L} \gamma^{\mu} \nu_{L}+\bar{d}_{L} \gamma^{\mu} u_{L} \\
J_{Z}^{\mu}= & \sum_{f} \bar{f} \gamma^{\mu}\left(T^{3}-\sin ^{2} \theta_{W} Q\right) f \\
= & \bar{\nu}_{L} \gamma^{\mu}\left(\frac{1}{2}\right) \nu_{L}+\bar{e}_{L} \gamma^{\mu}\left(-\frac{1}{2}+\sin ^{2} \theta_{W}\right) e_{L}+\bar{e}_{R} \gamma^{\mu}\left(\sin ^{2} \theta_{W}\right) e_{R} \\
& +\bar{u}_{L} \gamma^{\mu}\left(\frac{1}{2}-\frac{2}{3} \sin ^{2} \theta_{W}\right) u_{L}+\bar{u}_{R} \gamma^{\mu}\left(-\frac{2}{3} \sin ^{2} \theta_{W}\right) u_{R} \\
& +\bar{d}_{L} \gamma^{\mu}\left(-\frac{1}{2}+\frac{1}{3} \sin ^{2} \theta_{W}\right) d_{L}+\bar{d}_{R} \gamma^{\mu}\left(\frac{1}{3} \sin ^{2} \theta_{W}\right) d_{R} \\
J_{E M}^{\mu}= & \bar{e} \gamma^{\mu}(-1) e+\bar{u} \gamma^{\mu}\left(+\frac{2}{3}\right) u+\bar{d}\left(-\frac{1}{3}\right) d .
\end{aligned}
$$

The first term in Eq.(1.38) is responsible for all charged-current interactions in which the lepton charge is changed by \pm 1 units, and $W_{\mu}^{+}$and $W_{\mu}^{-}$represent, respectively, $W^{+}$and $W^{-}$gauge bosons. The last two terms in Eq.(1.38) generate interactions mediated by the neutral gauge boson $Z^{0}$, and by the photon, denoted here by the gauge field $A_{\mu}$.

The Lagrangian in Eq.(1.38) leads to the effective Hamiltonian of weak interaction defined by

$$
S=\frac{(-i)^{2}}{2 !} \int d^{4} x d^{4} y T[\mathcal{L}(x) \mathcal{L}(y)]=-\int \mathcal{H}_{e f f}(x) d^{4} x
$$

where $S$ is the S-matrix in the second order in $g$ and $T[\cdots]$ denotes the time ordered product. The result, which is valid in the region where the momentum transfer squared, $q^{2}$, satisfies $\left|q^{2}\right| \ll m_{W}^{2}, m_{Z}^{2}$, is

$$
\mathcal{H}_{e f f}=\frac{g^{2}}{4 m_{W}^{2}}\left(J_{W}^{\mu+} J_{W \mu}^{-}+\text {h.c. }\right)+\frac{g^{2}}{8 m_{Z}^{2} \cos ^{2} \theta_{W}} J_{Z}^{\mu} J_{Z \mu} \text {. }
$$


The first factor is often written in terms of the Fermi constant $G_{F}$, defined as

$$
\frac{G_{F}}{\sqrt{2}}=\frac{g^{2}}{8 m_{W}^{2}} .
$$

This constant gives the strength of the weak interactions at energies much less than $m_{W}$. We will show later that the coefficient of the neutral current term in $\mathcal{H}_{\text {eff }}$ of Eq.(1.41) is $G_{F} / \sqrt{2}$.

In the literature, the neutral-current $J_{Z}^{\mu}$ is often written as

$$
J_{Z}^{\mu}=\frac{1}{2} \bar{f} \gamma^{\mu}\left(c_{V}^{f}-c_{A}^{f} \gamma^{5}\right) f
$$

where the vector and axial-vector couplings for different particles are listed in Table 1.1.

\begin{tabular}{ccc}
\hline$f$ & $c_{V}$ & $c_{A}$ \\
\hline$\nu_{e}, \nu_{\mu}, \nu_{\tau}$ & $\frac{1}{2}$ & $\frac{1}{2}$ \\
$e^{-}, \mu^{-}, \tau^{-}$ & $-\frac{1}{2}+2 \sin ^{2} \theta_{W}$ & $-\frac{1}{2}$ \\
$u, c, t$ & $\frac{1}{2}-\frac{4}{3} \sin ^{2} \theta_{W}$ & $\frac{1}{2}$ \\
$d, s, b$ & $-\frac{1}{2}+\frac{2}{3} \sin ^{2} \theta_{W}$ & $-\frac{1}{2}$ \\
\hline
\end{tabular}

Table 1.1: Neutral vector and axial vector couplings in the Standard Model

We have demonstrated the massless gauge bosons are required to preserve the $S U(2) \otimes U(1)$ local gauge invariance. But in the real world, the gauge bosons that mediate the weak interactions are massive. They can acquire mass through spontaneous Symmetry Breaking. To break the symmetry spontaneously, we introduce a scalar field or so-called Higgs sector whose Lagrangian is given by

$$
\mathcal{L}_{\phi}=\frac{1}{2} \mid\left(\left.D_{\mu} \phi\right|^{2}+\mu^{2} \phi^{\dagger} \phi-\frac{\lambda}{2}\left(\phi^{\dagger} \phi\right)^{2},\right.
$$


where $\lambda>0$ and $\mu^{2}>0$ and

$$
\phi \equiv\left(\begin{array}{c}
\phi^{+} \\
\phi^{0}
\end{array}\right)
$$

The scalar field $\phi$ will acquire a vacuum expectation value at the stationary point of the Lagrangian

$$
\langle\phi\rangle^{\dagger}\langle\phi\rangle \equiv v^{2}=\frac{\mu^{2}}{\lambda}
$$

We can always perform an $S U(2) \otimes U(1)$ gauge transformation so that $\phi^{+}=0$ and $\phi^{0}$ is Hermitian. We can assume the field $\phi$ acquires a vacuum expectation value (VEV) of the form

$$
\langle\phi\rangle=\left(\begin{array}{l}
0 \\
v
\end{array}\right) .
$$

We can expand the Lagrangian (1.44) about the VEV (1.47). Decompose the Higgs field $\phi$ as

$$
\phi=\left(\begin{array}{c}
0 \\
v+H
\end{array}\right)
$$

where $H$ is a real scalar field. We assign the Higgs field a hypercharge $+1 / 2$ (or equivalently we assume the Higgs field is neutral). The Lagrangian (1.44) becomes

$$
\begin{aligned}
\mathcal{L}_{\phi}= & \frac{1}{2}\left|\left\{\partial_{\mu} H+i g(v+H) T^{a} A_{\mu}^{a}-i g^{\prime} \frac{1}{2}(v+H) B_{\mu}\right\}\left(\begin{array}{c}
0 \\
1
\end{array}\right)\right|^{2} \\
& +\mu^{2}(v+H)^{2}-\frac{\lambda}{2}(v+H)^{4} \\
= & \frac{1}{2}\left(\partial^{\mu} H\right)\left(\partial_{\mu} H\right)-\frac{1}{2}\left(2 \lambda v^{2}\right) H^{2}+\frac{1}{2}\left(\frac{g v}{2}\right)^{2} W^{+\mu} W_{\mu}^{+\dagger} \\
& +\frac{1}{2}\left(\frac{g v}{2}\right)^{2} W^{-\mu} W_{\mu}^{-\dagger}+\frac{1}{2}\left(\frac{v \sqrt{g^{2}+g^{\prime 2}}}{2}\right)^{2} Z^{0 \mu} Z_{\mu}^{0}+\cdots
\end{aligned}
$$

which implies: 
- $H$ survives as a physical particle, called the Higgs particle, with mass given by

$$
m_{H}=\sqrt{2 \lambda} v .
$$

- $W^{ \pm}$now acquire mass:

$$
m_{W^{ \pm}}=\frac{g v}{2} .
$$

- $Z^{0}$ acquires mass:

$$
m_{Z^{0}}=\frac{\sqrt{g^{2}+g^{\prime 2}}}{2} v
$$

From Eqs.(1.35), (1.51) and (1.52), we find that

$$
\left(\frac{m_{W}}{m_{Z}}\right)^{2}=\cos ^{2} \theta_{W}
$$

The coefficient in the second term in $\mathcal{H}_{\text {eff }}$ of Eq.(1.41) now becomes

$$
\frac{g^{2}}{8 m_{Z}^{2} \cos ^{2} \theta_{W}}=\frac{g^{2}}{8 m_{W}^{2}\left[m_{Z}^{2} / m_{W}^{2} \cos ^{2} \theta_{W}\right]}=\frac{G_{F}}{2} .
$$

That is, in the effective four-point interaction described by $\mathcal{H}_{e} f f$, the charged and neutral currents have the same strength; i.e.,

$$
\mathcal{H}_{\text {eff }}=\frac{G_{F}}{\sqrt{2}}\left(2 J_{W}^{\mu+} J_{W \mu}^{-}+\text {h.c. }+J_{Z}^{\mu} J_{Z \mu}\right) .
$$

In order to write the Fermion mass terms, we must now make some assumption about the mechanism of symmetry breaking. We want this mechanism to give masses not only to the $W^{ \pm}$and $Z^{0}$, but to the electron as well. The only way that this is possible in a renormalizable weakly-coupled theory is to have a scalar field coupled without derivatives to $\bar{l}_{R}$ and $l_{L}$ (and also $\bar{l}_{L}$ and $l_{R}$ ). Then the $S U(2)_{L} \otimes U(1)$ invariance requires that the scalar be an $S U(2)_{L}$ doublet. We thus assume a "Yukawa" 
coupling

$$
\mathcal{L}_{\phi e}=-f_{e} \overline{\left(\begin{array}{c}
\nu_{e} \\
e
\end{array}\right)_{L}}\left(\begin{array}{c}
\phi^{+} \\
\phi^{0}
\end{array}\right) e_{R}+\text { h.c. }
$$

If we replace $\phi$ in this expression by its vacuum expectation value (1.47), we obtain

$$
\mathcal{L}_{\phi e}=-f_{e} v \bar{e}_{L} e_{R}+\text { h.c. }
$$

This is a mass term for the electron and it couples the right-handed electron to the left-handed electron. The size of the mass is set by the vacuum expectation value of $\phi$, rescaled by the new dimensionless coupling

$$
m_{e}=f_{e} v
$$

The neutrino does not have a similar term to the electron as there is no $\nu_{R}$ singlet in the Standard Model. Therefore, according to the Standard Model, neutrinos must be massless. However, the neutrino oscillation experiments have provided compelling evidence that neutrinos do have mass. The Standard Model has to be extended in order to accommodate neutrino mass.

\subsection{Massive Neutrinos}

The Standard Model itself is based on the gauge group $S U(2)_{L} \otimes U(1)_{Y}$. But this fixes only the gauge bosons of the model. The fermions and Higgs contents have to be chosen somewhat arbitrarily. In the Standard Model, these choices are made in such a way that the neutrinos are massless. However, even with the same gauge group as the Standard Model, one can conjecture extra fermions or Higgs bosons in the model so that the model predicts massive neutrinos. In this section, we will start with the discussion of Dirac and Majorana masses and then introduce the Seesaw Mechanism 
and neutrino mixing.

\subsubsection{Dirac and Majorana Masses}

The Standard Model contains left and right chiral projections of all fermions except the neutrinos. To extend the Standard Model in order to accommodate a nonzero neutrino mass, we add right-handed neutral fields $\nu_{l}^{R}$ corresponding to each charged lepton $l$. They are assumed to be $S U(2)_{L}$ singlets. Their hypercharge $Y$ is 0 according to Eq.(1.32). We can couple the right-handed singlets to the left-handed doublets and Higgs doublets through the Yukawa coupling

$$
-\mathcal{L}_{\phi \nu}=f_{\nu} \bar{\psi}_{l L} \phi \nu_{R}+\text { h.c. }
$$

If we replace $\phi$ by its vacuum expectation value $v$, we obtain the following mass term

$$
\begin{aligned}
-\mathcal{L}_{D} & =f_{\nu} v \bar{\nu}_{L} \nu_{R}+\text { h.c. } \\
& \equiv m_{D} \bar{\nu}_{L} \nu_{R}+\text { h.c. }
\end{aligned}
$$

This mass term is called a Dirac mass term and is constructed out of a chirally lefthanded neutrino field $\nu_{L}$ and a chirally right-handed one $\nu_{R}$. The neutrino mass is

$$
m_{D}=f_{\nu} v
$$

which is set by the vacuum expectation value of $\phi$ and a new dimensionless coupling constant $f_{\nu}$. The problem with this model is that it provides no answer to the question about the lightness of neutrinos, namely why $f_{\nu_{e}}$ is so much smaller than $f_{e}$ even though $\nu_{e}$ and $e$ are in the same leptonic generation.

We can also construct mass terms out of $\nu_{L}$ alone or $\nu_{R}$ alone [31]. These mass 
terms are called Majorana mass terms. We can have the "left-handed Majorana mass"

$$
-\mathcal{L}_{M L}=-\frac{m_{L}}{2} \overline{\left(\nu_{L}\right)^{C}} \nu_{L}+\text { h.c. },
$$

or we can have the "right-handed Majorana mass"

$$
-\mathcal{L}_{M R}=-\frac{m_{R}}{2} \overline{\left(\nu_{R}\right)^{C}} \nu_{R}+\text { h.c. . }
$$

In these expressions, $m_{L}$ and $m_{R}$ are mass parameters, and for any field $\psi, \psi^{C}$ is the corresponding charge-conjugate field. In terms of $\psi, \psi^{C}=C \bar{\psi}^{T}$, where $C$ is the charge conjugation matrix, and $T$ denotes transposition. $\psi^{C}$ represents the antiparticle field of $\psi$. In the Dirac bases $C=\gamma^{2}$ (up to an arbitrary phase). Note $\left(\nu_{L}\right)^{C}$ is a right-handed neutrino field and $\left(\nu_{R}\right)^{C}$ is a left-handed neutrino field.

A Dirac mass term turns a neutrino into a neutrino, or an antineutrino into an antineutrino, while a Majorana mass term converts a neutrino into an antineutrino, or vice versa. Thus, Dirac mass terms conserve the lepton number $L$ that distinguishes leptons from antileptons, while Majorana mass terms do not. The quantum number $L$ is also conserved by the Standard Model couplings of neutrinos to other particles. Thus, if we assume that the interactions between neutrinos and other particles are well described by these Standard Model couplings then any $L$ nonconservation that we might observe in neutrino experiments would have to arise from Majorana mass terms, not from interactions.

An electrically charged fermion such as a quark cannot have a Majorana mass term, because such a term would convert it into an antiquark, in violation of electric charge conservation. However, for the electrically neutral neutrinos, Majorana mass terms are not only allowed but rather likely, given that the neutrinos are now known to be particles with mass. 


\subsubsection{Seesaw Mechanism and Neutrino Mixing}

Suppose that a neutrino has a Dirac mass, as the quarks and charged leptons do, and also a right-handed Majorana mass. Then its total mass term $\mathcal{L}_{m \nu}$ is

$$
-\mathcal{L}_{m \nu}=m_{D} \overline{\nu_{L}} \nu_{R}+\frac{1}{2} m_{R} \overline{\left(\nu_{R}\right)^{C}} \nu_{R}+\text { h.c. }
$$

where $m_{D}$ is a Dirac mass, and $m_{R}$ is a Majorana mass. We define

$$
\nu \equiv\left(\begin{array}{c}
\nu_{L} \\
\nu_{R}^{C}
\end{array}\right)
$$

where $\nu_{R}^{C} \equiv\left(\nu_{R}\right)^{C}$ is a left-handed field. Eq.(1.64) can be rewritten as

$$
-\mathcal{L}_{m \nu}=\frac{1}{2} \overline{\nu^{C}} \mathcal{M} \nu+\text { h.c. },
$$

where

$$
\mathcal{M}=\left(\begin{array}{cc}
0 & m_{D} \\
m_{D} & m_{R}
\end{array}\right)
$$

and $m_{D}$ and $m_{R}$ are simply numbers. Here, we have used the identity

$$
\overline{\left(\nu_{L}\right)^{C}} m_{D}\left(\nu_{R}\right)^{C}=\overline{\nu_{L}} m_{D} \nu_{R} .
$$

$\mathcal{M}$ is referred to as the neutrino mass matrix. For the sake of simplicity, let us assume that both $m_{D}$ and $m_{R}$ are real and $m_{R}>0$. Now choose an orthogonal matrix

$$
O=\left(\begin{array}{cc}
\cos \theta & -\sin \theta \\
\sin \theta & \cos \theta
\end{array}\right)
$$


with

$$
\tan 2 \theta=2 m_{D} / m_{R}
$$

Then

$$
O \mathcal{M} O^{T}=\left(\begin{array}{cc}
-m_{1} & 0 \\
0 & m_{2}
\end{array}\right)
$$

where

$$
m_{1,2}=\frac{1}{2}\left(\sqrt{m_{R}^{2}+4 m_{D}^{2}} \mp m_{R}\right) .
$$

The elements of the diagonal matrix in Eq.(1.71) are not all non-negative and therefore cannot be interpreted as the masses of physical fields. But there is a work-around. Let us diagonalize the mass matrix (1.67) with the following unitary matrix

$$
U=K O=\left(\begin{array}{cc}
i & 0 \\
0 & 1
\end{array}\right)\left(\begin{array}{cc}
\cos \theta & -\sin \theta \\
\sin \theta & \cos \theta
\end{array}\right)
$$

We now have

$$
U \mathcal{M} U^{T}=\left(\begin{array}{cc}
m_{1} & 0 \\
0 & m_{2}
\end{array}\right)
$$

To recast $\mathcal{L}_{m \nu}$ in terms of mass eigenfields, we define the two-component column vector $\nu_{L}$ by

$$
\nu_{L}^{\prime} \equiv\left(U^{T}\right)^{-1}\left(\begin{array}{c}
\nu_{L} \\
\nu_{R}^{C}
\end{array}\right)
$$

The column-vector field $\nu_{L}$ is chirally left-handed, since the charge conjugate of a field with a given chirality always has the opposite chirality. We then define the two-component field $\nu$, with components $\nu_{1}$ and $\nu_{2}$, by

$$
\nu^{\prime} \equiv \nu_{L}^{\prime}+\left(\nu_{L}^{\prime}\right)^{C} \equiv\left(\begin{array}{c}
\nu_{1} \\
\nu_{2}
\end{array}\right)
$$


To write down $\nu_{1}$ and $\nu_{2}$ explicitly

$$
\begin{aligned}
& \nu_{1}=i \cos \theta\left(\nu_{L}^{C}-\nu_{L}\right)+i \cos \theta\left(\nu_{R}^{C}-\nu_{R}\right) \\
& \nu_{2}=\sin \theta\left(\nu_{L}+\nu_{L}^{C}\right)+\cos \theta\left(\nu_{R}+\nu_{R}^{C}\right) .
\end{aligned}
$$

Since $\left(\nu_{L}^{C}\right)^{C}=\nu_{L},\left(\nu_{R}^{C}\right)^{C}=\nu_{R}$, this immediately proves

$$
\nu_{1}^{C}=-\nu_{1}, \nu_{2}^{C}=\nu_{2},
$$

which implies that $\nu_{1}$ and $\nu_{2}$ are their own anti-particles, or equivalently, they are self-conjugate particles.

There are three (Weyl, Dirac and Majorana) types of spin-half fermion fields. Massless fermions are Weyl particles. Massive spin-half objects can be of either Dirac or Majorana type. Fermions that are distinct from their anti-particles are known as Dirac particles. Ferminos that are identical to their own anti-particles are known as Majorana particles. All charged fermions are Dirac particles as a consequence of the electric charge conservation. Conservation of the lepton number is decidedly less fundamental than the electric charge conservation. Without lepton number conservation, neutrinos do not hold any additive internal "charge" and can be identical to their own anti-particles. So the neutrinos can be Majorana particles. The eigenstates of the combined Dirac-Majorana mass term $\mathcal{L}_{m \nu}$ are Majorana neutrinos $\left(\nu_{1}\right.$ and $\left.\nu_{2}\right)$. For $N$ generations, one obtains $2 N$ Majorana particles in general. The mass terms can be rewritten as

$$
-\mathcal{L}_{m \nu}=\frac{1}{2} m_{1} \overline{\nu_{1 L}} \nu_{1 R}+\frac{1}{2} m_{2} \overline{\nu_{2 L}} \nu_{2 R}+\text { h.c. }=\frac{1}{2} m_{1} \overline{\nu_{1}} \nu_{1}+\frac{1}{2} m_{2} \overline{\nu_{2}} \nu_{2}
$$


The masses of neutrinos $\nu_{1}$ and $\nu_{2}$ are $m_{1}$ and $m_{2}$, respectively ${ }^{2}$. It is natural to suppose that the Dirac mass $m_{D}$ of our neutrino is of the same order of magnitude as the Dirac masses of the lightest quarks and charged leptons, since in the Standard Model all of these Dirac masses arise from couplings to the same Higgs field. Furthermore, nothing in the Standard Model requires the right-handed Majorana mass $m_{R}$ to be small. If this mass is large: $m_{R} \gg m_{D}$, we have

$$
m_{1} \simeq m_{D}^{2} / m_{R} \text { and } m_{2} \simeq m_{R}
$$

Thus if $m_{R} \gg m_{D}$, it follows that $m_{1} \ll m_{D}$, which means that the neutrino is much lighter than the charged fermions. If we identify $\nu_{1}$ as one of the light neutrinos, we have an elegant explanation of why it is so light. Of course, there is another neutrino whose mass, $m_{R}$ is much larger than the charged fermion masses. This mechanism of making one particle light at the expense of making another one heavy is called the see-saw mechanism. Interestingly, if $m_{R}$ is just a bit below the grand unification scale, say $m_{R} \sim 10^{15} \mathrm{GeV}$, and $m_{D} \sim m_{\text {top }} \simeq 175 \mathrm{GeV}$, then from Eq.(1.80) $m_{1} \sim 3 \times 10^{-2}$ $\mathrm{eV}$. This is right in the range of neutrino mass suggested by the experiments on atmospheric neutrino oscillation which we will discuss later in this chapter.

So far, we have analyzed the simplified case in which there is only one light neutrino and one heavy neutral lepton. The Seesaw Mechanism can be extended to accommodate three leptonic generations. We skip the details of this analysis and only discuss the results. We can construct the mass term $\mathcal{L}_{m \nu}$ with 3 Dirac masses and 3 Majorana masses. By diagonalizing the mass matrix, one gets 3 light neutrinos $\nu_{1,2,3}^{\text {Light }}$ and 3 heavy neutrinos $\nu_{1,2,3}^{\text {Heavy }}$. In neutrino experiments at low energies, it is only the

\footnotetext{
${ }^{2}$ The mass term for a Dirac fermion $f$ of mass $m_{f}$ is $-m_{f} \bar{f} f$. But the mass term for a Majorana neutrino $\nu$ of mass $m_{\nu}$ is $-(1 / 2) m_{\nu} \bar{\nu} \nu[31]$.
} 
light neutrinos that play a significant role. From the analogue of Eq.(1.75), we have

$$
\nu_{L \alpha}=\sum_{i=1}^{3}\left(U_{\alpha i} \nu_{L i}^{\text {Light }}+V_{\alpha i} \nu_{L i}^{\text {Heavy }}\right) \simeq \sum_{i=1}^{3} U_{\alpha i} \nu_{L i}^{\text {Light }}
$$

where $\alpha=e, \mu$, or $\tau$ and $\nu_{\alpha}$ is a weak-eigenstate. $U$ and $V$ are two unitary matrices and $V \ll U$ is satisfied if the Dirac mass $m_{D}$ is much smaller than the Majorana mass $m_{R}$. Denote $\nu_{i}=\nu_{i}^{\text {Light }}$ for simplicity, we have

$$
\nu_{\alpha}=\sum_{i=1}^{3} U_{\alpha i} \nu_{i}
$$

which means the weak-eigenstate neutrino field is a linear combination of neutrino fields with definite masses. This phenomenon is known as neutrino mixing and the unitary matrix $U$ is known as the leptonic mixing matrix. In discussion of neutrino oscillations, we start with a flavor (or weak) state, $\left|\nu_{\alpha}\right\rangle$, produced via weak interactions. Since $\nu_{\alpha}^{\dagger}$ contains the creation operator $a^{\dagger}$ which creates $\left|\nu_{\alpha}\right\rangle$ from the vacuum state $|0\rangle$, we obtain, from Eq.(1.82),

$$
\left|\nu_{\alpha}\right\rangle=\sum_{i=1}^{3} U_{\alpha i}^{*}\left|\nu_{i}\right\rangle
$$

which means the weak-eigenstate is a superposition of mass eigenstates.

The charged-current weak interaction in Eq.(1.38) now takes the form

$$
\mathcal{L}_{W}=\frac{g}{\sqrt{2}} W_{\mu}^{-} \sum_{\substack{\alpha=e, \mu, \tau \\ i=1,2,3}} \bar{l}_{L \alpha} \gamma^{\mu} U_{\alpha i} \nu_{L i}+\text { h.c. }
$$

Here we do not take into account the charged leptonic mixing, i.e., we assume the weak-eigenstate charged lepton is identical to the mass eigenstate. However, even if charged leptonic mixing does occur, we can still absorb its mixing matrix into the 
matrix $U$.

What neutrino mixing means may be understood by considering the leptonic decays $W^{+} \rightarrow \nu_{i}+\bar{l}_{\alpha}$ of the $W$ boson. Here, $\alpha=e, \mu$, or $\tau$ and $\nu_{\alpha}$ is referred to as the charged lepton of flavor $\alpha$. Mixing means that in the $W^{+}$decays to the particular charged lepton $\bar{l}_{\alpha}$, the accompanying neutrino mass eigenstate is not always the same $\nu_{i}$, but can be any of the different $\nu_{i}(\mathrm{i}=1,2,3)$. The amplitude for $W^{+}$decay to produce the specific combination $\nu_{i}+\bar{l}_{\alpha}$ is denoted by $U_{\alpha i}$.

The evidence for neutrino masses and mixing is the observation that neutrinos can change from one type, or "flavor", to another. The change of neutrino flavor, or neutrino "oscillation" as it is called, is a quantum-mechanical effect. This discussion of neutrino oscillations in this section follows [33]. Suppose a neutrino $\nu_{\alpha}$ is created together with a charged lepton $\bar{l}_{\alpha}$. The neutrino then travels a distance $L$ to a detector. There, it interacts with a target and produces a second charged lepton $l_{\beta}$ of flavor $\beta$. Thus, at the time of its interaction in the detector, the neutrino is a $\nu_{\beta}$. If $\beta \neq \alpha$, then, during its journey to the detector, the neutrino has morphed from a $\nu_{\alpha}$ into a $\nu_{\beta}$. The amplitude of neutrino oscillation is

$$
\operatorname{Amp}\left(\nu_{\alpha} \rightarrow \nu_{\beta}\right)=\sum_{i} U_{\alpha i}^{*} \operatorname{Prop}\left(\nu_{i}\right) U_{\beta i}
$$

We now calculate $\operatorname{Prop}\left(\nu_{i}\right)$. The propagation of a neutrino $\nu_{i}$ with a mass $m_{i}$ can be expressed as a plane wave

$$
\left|\nu_{i}(t)\right\rangle=e^{-i\left(E_{i} t-p_{i} L\right)}\left|\nu_{i}(0)\right\rangle
$$

where $t$ and $L$ are the time and distance the neutrino travels and $E_{i}$ and $p_{i}$ are its energy and momentum. The only components of a neutrino beam that contribute coherently to a neutrino oscillation signal are components that have the same energy 
[34]. In particular, the different mass eigenstate components of a beam that contribute coherently to the oscillation signal must have the same energy, $E$.

At energy $E$, mass eigenstate $\nu_{i}$, with mass $m_{i}$, has a momentum $p_{i}$ given by

$$
p_{i}=\sqrt{E^{2}-m_{i}^{2}} \simeq E-\frac{m_{i}^{2}}{2 E} .
$$

Here, we have used the fact that, given the extreme lightness of neutrinos, $m_{i}^{2} \ll E^{2}$ for any realistic energy $E$. We can see that at energy $E$ the phase in $\nu_{i}(t)$ is given by

$$
E_{i} t-p_{i} L \simeq E(t-L)+\frac{m_{i}^{2}}{2 E} L .
$$

In this expression, the phase $E(t-L)$ is irrelevant since it is common to all the interfering mass eigenstates. Thus we may take

$$
\operatorname{Prop}\left(\nu_{i}\right)=e^{-i m_{i}^{2} \frac{L}{2 E}} .
$$

Using this result, the amplitude for a neutrino to change from a $\nu_{\alpha}$ into a $\nu_{\beta}$ while traveling a distance $L$ through vacuum with energy $\mathrm{E}$ is given by

$$
\operatorname{Amp}\left(\nu_{\alpha} \rightarrow \nu_{\beta}\right)=\sum_{i} U_{\alpha i}^{*} e^{-i m_{i}^{2} \frac{L}{2 E}} U_{\beta i}
$$

This expression holds for any number of flavors and mass eigenstates. Squaring it, we find that the probability $\mathrm{P}\left(\nu_{\alpha} \rightarrow \nu_{\beta}\right)$ for $\nu_{\alpha} \rightarrow \nu_{\beta}$ is given by

$$
\begin{aligned}
\mathrm{P}\left(\nu_{\alpha} \rightarrow \nu_{\beta}\right)= & \left|\operatorname{Amp}\left(\nu_{\alpha} \rightarrow \nu_{\beta}\right)\right|^{2} \\
= & \delta_{\alpha \beta}-4 \sum_{i>j} \mathfrak{R}\left(U_{\alpha i}^{*} U_{\beta i} U_{\alpha j} U_{\beta j}^{*}\right) \sin ^{2}\left(\Delta m_{i j}^{2} \frac{L}{4 E}\right) \\
& +2 \sum_{i>j} \Im\left(U_{\alpha i}^{*} U_{\beta i} U_{\alpha j} U_{\beta j}^{*}\right) \sin \left(\Delta m_{i j}^{2} \frac{L}{2 E}\right)
\end{aligned}
$$


where

$$
\Delta m_{i j}^{2}=m_{i}^{2}-m_{j}^{2} .
$$

A common parametrization of $U$ in terms of mixing angles and phases is

$$
\begin{aligned}
U & =\left[\begin{array}{ccc}
1 & 0 & 0 \\
0 & c_{23} & s_{23} \\
0 & -s_{23} & c_{23}
\end{array}\right] \times\left[\begin{array}{ccc}
c_{13} & 0 & s_{13} e^{-i \delta} \\
0 & 1 & 0 \\
-s_{13} e^{i \delta} & 0 & c_{13}
\end{array}\right] \times\left[\begin{array}{ccc}
c_{12} & s_{12} & 0 \\
-s_{12} & c_{12} & 0 \\
0 & 0 & 1
\end{array}\right] \times\left[\begin{array}{ccc}
e^{i \alpha_{1} / 2} & 0 & 0 \\
0 & e^{i \alpha_{2} / 2} & 0 \\
0 & 0 & 1
\end{array}\right] \\
& =\left[\begin{array}{ccc}
c_{12} c_{13} \\
s_{12} c_{13} & s_{13} e^{-i \delta} \\
-s_{12} c_{23}-c_{12} s_{23} s_{13} e^{i \delta} & c_{12} c_{23}-s_{12} s_{23} s_{13} e^{i \delta} & s_{23} c_{13} \\
s_{12} s_{23}-c_{12} c_{23} s_{13} e^{i \delta} & -c_{12} s_{23}-s_{12} c_{23} s_{13} e^{i \delta} & c_{23} c_{13}
\end{array}\right] \times\left[\begin{array}{ccc}
e^{i \alpha_{1} / 2} & 0 & 0 \\
0 & e^{i \alpha_{2} / 2} & 0 \\
0 & 0 & 1
\end{array}\right]
\end{aligned}
$$

Here, $c_{i j} \equiv \cos \theta_{i j}$ and $s_{i j} \equiv \sin \theta_{i j}$, where $\theta_{12}, \theta_{13}$, and $\theta_{23}$ are the three mixing angles, and $\delta, \alpha_{1}$, and $\alpha_{2}$ are the three CP-violating phases. The phase $\delta$ is referred to as a Dirac phase where the phases $\alpha_{1}$ and $\alpha_{2}$ are known as Majorana phases. This matrix is sometimes referred to as the Maki-Nakagawa-Sakata matrix, or as the Pontecorvo-Maki-Nakagawa-Sakata (PMNS) matrix, in recognition of the pioneering contributions of these physicists to the physics of mixing and oscillation [35, 36].

An important special case is the case where only two different neutrinos are important. The two-neutrino approximation is a fairly accurate description of a number of experiments. Suppose that only two mass eigenstates, which we shall call $\nu_{1}$ and $\nu_{2}$, and two corresponding flavor states, which we shall call $\nu_{\alpha}$ and $\nu_{\beta}$ are significant. The phase factors then can be shown to have no effect on oscillations. The mixing matrix $U$ takes the simple form

$$
U=\left(\begin{array}{rr}
\cos \theta & \sin \theta \\
-\sin \theta & \cos \theta
\end{array}\right) .
$$


For $\beta \neq \alpha$, the neutrino oscillation probability is

$$
\mathrm{P}\left(\nu_{\alpha} \rightarrow \nu_{\beta}\right)=\sin ^{2} 2 \theta \sin ^{2}\left(\Delta m^{2} \frac{L}{4 E}\right)
$$

where $\Delta m^{2} \equiv m_{1}^{2}-m_{2}^{2}$. In addition, the probability that the neutrino does not change flavor is, as usual, unity minus the probability that it does change flavor.

If neutrinos pass through enough matter between their source and a target detector, then their coherent forward scattering from particles in the matter can significantly modify their oscillation pattern. Flavor change in matter that grows out of an interplay between flavor-nonchanging neutrino-matter interactions and neutrino mass and mixing is known as the Mikheyev-Smirnov-Wolfenstein (MSW) effect [80, 81, 82].

Matter is composed of nucleons (or quarks) and electrons. The contribution of nucleons (or quarks) to the forward scattering amplitude is described by the neutral current ( $Z^{0}$ exchange); it is identical for all neutrino flavors thus it has no effect on the neutrino oscillations. For electrons the situation is different; the electron neutrinos interact with electrons via both the neutral current and the charged current $\left(W^{+}\right.$ exchange). All other neutrino flavors interact only via the neutral current, so their interaction is different in magnitude than that of the electron neutrinos. Coherent forward scattering by electrons via $W$ exchange gives rise to an extra interaction potential energy $V$ possessed by electron neutrinos in matter. From the Standard Model, we find that

$$
V=+\sqrt{2} G_{F} N_{e},
$$

where $G_{F}$ is the Fermi coupling constant and $N_{e}$ is the number of electrons per unit volume. This interaction potential energy changes sign if we replace the $\nu_{e}$ in the beam by $\bar{\nu}_{e}$. This potential gives rise to an effective mixing and mass matrix. The MSW effect has a significant impact on the oscillations of solar neutrinos. High energy ( $>5 \mathrm{MeV}$ ) electron neutrinos (e.g. ${ }^{7}$ Be neutrinos) produced in the core of the 
sun leave its surface as almost pure mass eigenstate $\nu_{2}$. This is an effect of neutrino adiabatic propagation through the high electron density in the sun ${ }^{3}$. Also, there will be an asymmetry between antineutrino oscillation and neutrino oscillation that is induced by matter effects. This asymmetry has nothing to do with genuine $\mathrm{CP}$ violation, and will have to be disentangled from the antineutrino-neutrino asymmetry that does come from genuine CP violation in order for us to be able to study the latter phenomenon. This antineutrino-neutrino asymmetry coming from matter effects can be utilized to understand the neutrino mass hierarchy. The neutrino mass hierarchy will be discussed in the next section.

\subsection{Neutrino Experiments}

Neutrinos have been a focus of experimental effort over the last decade [37]. The theoretical framework with which we describe the three known neutrinos has crystallized. The three neutrino mass eigenstates, conventionally known as $\nu_{1}, \nu_{2}$, and $\nu_{3}$ are related to the three flavor eigenstates $\nu_{e}, \nu_{\mu}$, and $\nu_{\tau}$ by a unitary matrix that can be conveniently broken into four parts. The mixing matrix is shown in Eq.(1.93).

Fig.1.1 shows graphicaly what we have learned so far about the neutrino masses from neutrino oscillation experiments. The overall mass scale is still unknown, but the lightest neutrino is constrained by tritium beta decay measurements to be less than about $2.2 \mathrm{eV} . \nu_{1}$ is defined as the mass eigenstate with the most $\nu_{e}$ in it and $\nu_{3}$ is defined as the mass eigenstate with the least $\nu_{e}$ in it. The solar neutrino experiments and the KamLand reactor experiment measure the squared mass difference between the 1 and 2 mass eigenstates to be $(7.0-9.1) \times 10^{-5} \mathrm{eV}^{2}$. The atmospheric neutrino measurements and long baseline experiments constrain the squared mass difference between the 2 and 3 mass eigenstates to be $(1.9-2.98) \times 10^{-3} \mathrm{eV}^{2}$. Both are $3 \sigma$

\footnotetext{
${ }^{3}$ Note neutrino oscillations are dominated by vacuum oscillations at low energies $(<2 \mathrm{MeV})$. A smooth transition is expected between the two different regimes
} 


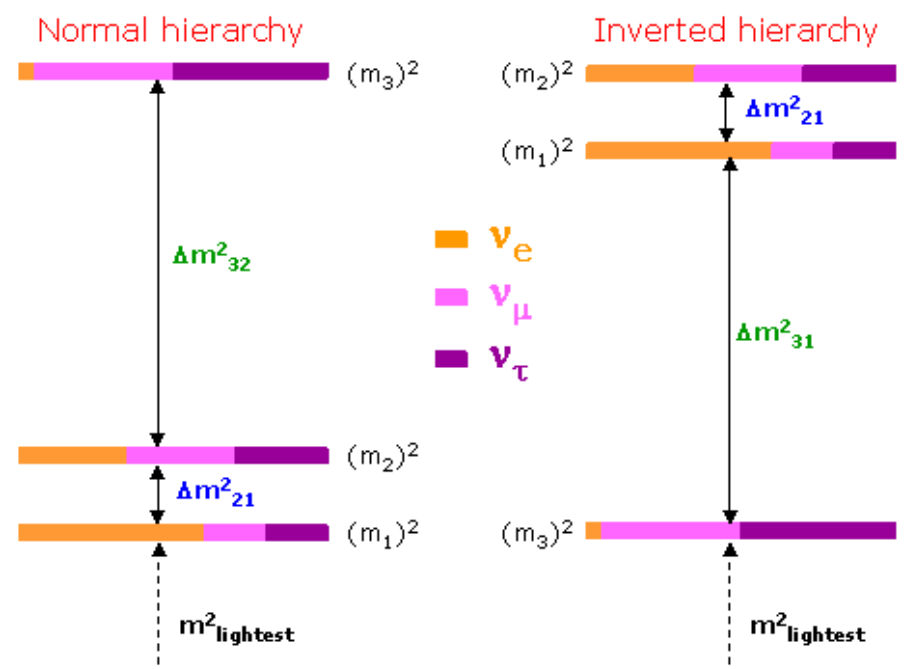

Figure 1.1: The two possible mass hierarchies for the three known neutrino flavors. Plot taken from [37].

ranges. The mass of $\nu_{2}$ is larger than the $\nu_{1}\left(m_{2}^{2}>m_{1}^{2}\right)$, which is determined from the Mikheyev-Smirnov-Wolfenstein (MSW) large mixing angle (LMA) solution to the solar neutrino problem. It is not determined, however, whether the mass of $\nu_{3}$ is larger or smaller than the $\nu_{1}$ and $\nu_{2}$ masses. These two scenarios are referred to as the normal hierarchy and inverted hierarchy respectively.

In this section, we will discuss experiments that measure the neutrino mixing angles and mass splittings in different sectors, i.e. the 12 Sector, the 23 Sector, the 13 Sector and the Mass Sector.

\subsubsection{The Solar Neutrino Problem}

The 12 Sector of neutrino mixing matrix comprises the mixing angle $\theta_{12}$ and the squared mass difference $\Delta m_{12}^{2}$ between $\nu_{2}$ and $\nu_{1}$. There are two types of experiments that have probed this sector: solar neutrino measurements and long baseline antineutrino experiments.

For almost 30 years, the Homestake Solar Neutrino Experiment in the Homestake 
Gold Mine in South Dakota attempted to measure neutrino fluxes from space; in particular, this experiment has been gathering information on solar neutrino fluxes. The results of this experiment have been checked against predictions made by standard solar models and it has been discovered that only one-third of the expected solar neutrino flux has been detected [38]. This discrepancy is known as the "Solar Neutrino Problem". Several other experiments, including Kamiokande II [39], Super-Kamiokande [41], GALLEX [42], SAGE [43], GNO [44], and Borexino [45], have noticed a definite neutrino shortfall. The measurements of solar neutrinos culminated in the Sudbury Neutrino Observatory (SNO) which used the deuterium in heavy water as a target for solar neutrinos. This enabled a measurement of both the electron neutrino flux through Charged Current interactions and the flux of all active neutrino flavors through Neutral Current interactions. Fig.1.2 summarizes the set of measurements from SNO and Super-K [40]. It plots the measured flux of electron neutrinos against the measured flux of muon and tau neutrinos. SNO finds that

$$
\frac{\phi\left(\nu_{e}\right)}{\phi\left(\nu_{e}\right)+\phi\left(\nu_{\mu, \tau}\right)}=0.340 \pm 0.023(\text { stat })_{-0.031}^{+0.029} \text { (syst) } .
$$

The conclusion is that the electron flavor neutrinos are oscillating into muon or tau neutrinos. The SNO measurement directly demonstrated neutrino oscillations in solar neutrinos. The results of the SNO experiment put the "Solar Neutrino Problem" to rest and, along with the KamLand reactor neutrino experiment, enable precision measurement of the 12 Sector parameters.

The KamLAND experiment is a reactor neutrino experiment which uses a 1 kiloton liquid scintillator detector located in the Kamioka mine in central Japan. Most of the $\bar{\nu}_{e}$ flux incident at KamLAND comes from nuclear plants at distance of 80 $350 \mathrm{~km}$ from the detector, making the average baseline of about 180 kilometers. By measuring reactor $\bar{\nu}_{e}$ 's, this experiment provided a sensitive probe of the solar neutrino 


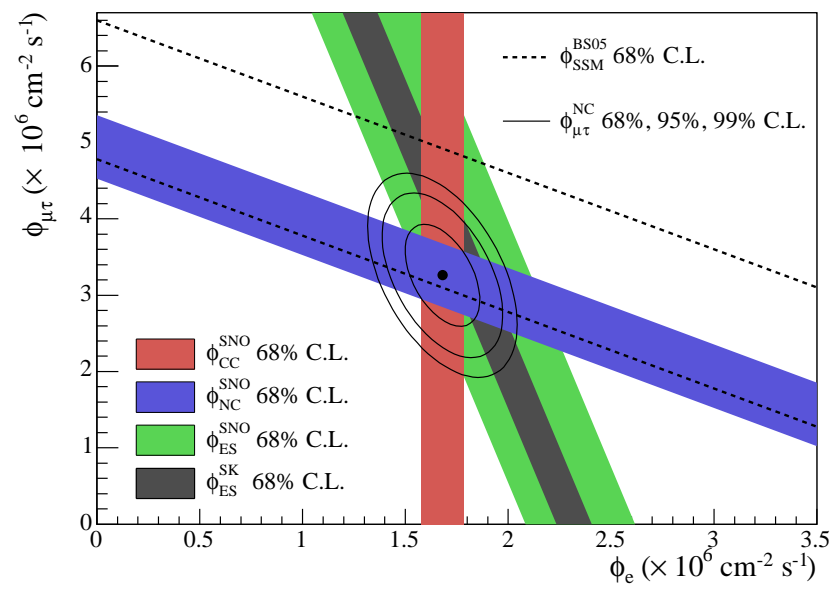

Figure 1.2: The SNO and Super-K results expressed as a measurement of the flux of $\mu+\tau$ neutrinos versus the flux of electron neutrinos. Plot taken from [40].

oscillations. The survival probability of electron neutrinos is

$$
P\left(\bar{\nu}_{e} \rightarrow \bar{\nu}_{e}\right) \approx 1-\sin ^{2} 2 \theta_{12} \sin ^{2}\left(1.27 \Delta m_{12}^{2} L / E_{\nu}\right)
$$

The KamLAND collaboration has for the first time measured the disappearance of neutrinos traveling to a detector from a power reactor. They observe a strong evidence for the disappearance of neutrinos during their flight over such distances, giving the first terrestrial confirmation of the solar neutrino anomaly and also establishing the oscillation hypothesis with man-produced neutrinos. Fig.1.3 shows the combined result of the KamLAND measurement and the solar neutrino experiments [46]. The combined analysis gives the $1 \sigma$ ranges [40]:

$$
\Delta m_{\text {solar }}^{2}=8.0_{-0.3}^{+0.4} \times 10^{-5} \mathrm{eV}^{2}, \theta_{\text {solar }}=33.9_{-1.6}^{+1.6} \text { degrees. }
$$



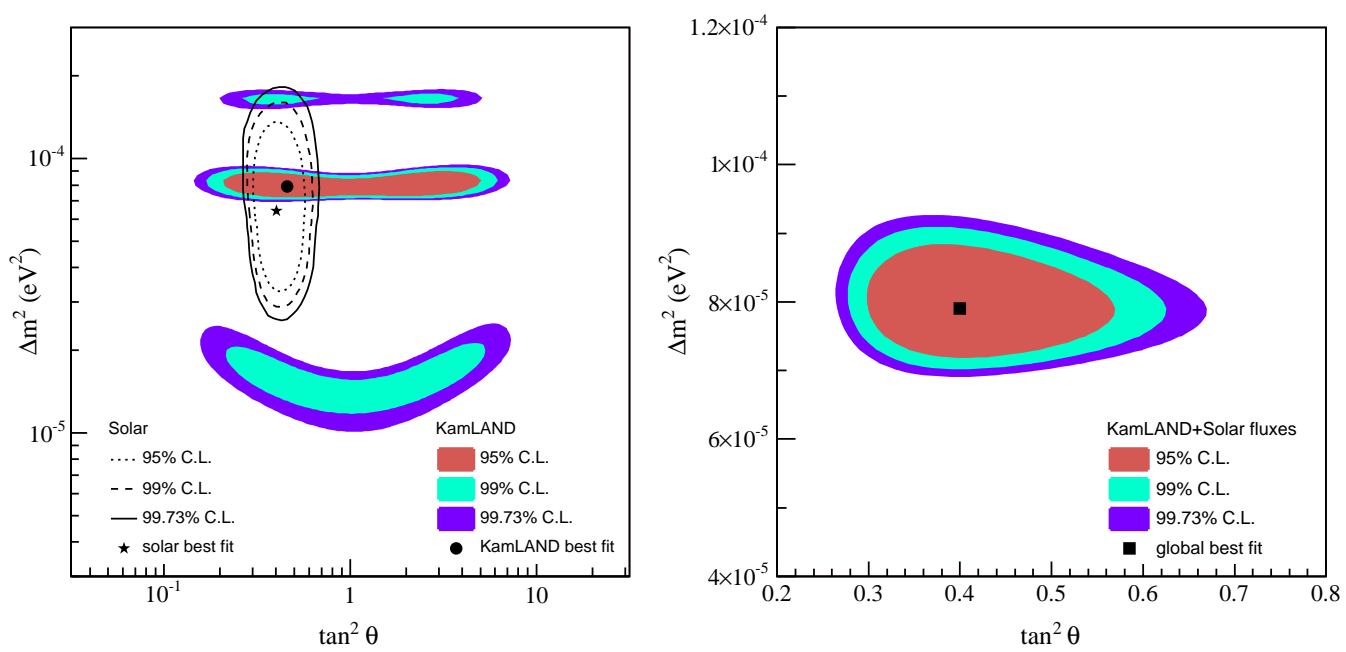

Figure 1.3: (a) Neutrino oscillation parameter allowed region from KamLAND antineutrino data (shaded regions) and solar neutrino experiments (lines). (b) Result of a combined two-neutrino oscillation analysis of KamLAND and the observed solar neutrino fluxes under the assumption of CPT invariance. Plot taken from [46].

\subsubsection{The Atmospheric Neutrino Anomaly}

The 23 Sector of neutrino mixing matrix comprises the mixing angle $\theta_{23}$ and the squared mass difference $\Delta m_{32}^{2}$ between $\nu_{3}$ and $\nu_{2}$. Measurements of muon neutrino survival probability probe this sector

$$
P\left(\nu_{\mu} \rightarrow \nu_{\mu}\right) \approx 1-\sin ^{2} 2 \theta_{23} \sin ^{2}\left(1.27 \Delta m_{32}^{2} L / E_{\nu}\right)
$$

There are two types of experiments that are sensitive to those oscillation parameters: experiments measuring the neutrinos produced when cosmic rays hit the atmosphere and experiments located several hundred kilometers from an accelerator source of muon neutrinos.

Atmospheric neutrinos are produced in the collision of primary cosmic rays (typically protons) with nuclei in the upper atmosphere. This creates a shower of hadrons, mostly pions. The principal sources of neutrinos are decays of pions, kaons, and 
muons. The decay chain from pions is

$$
\begin{aligned}
\pi^{ \pm} \rightarrow & \mu^{ \pm}+\nu_{\mu}\left(\bar{\nu}_{\mu}\right) \\
& \mu^{ \pm} \rightarrow e^{ \pm}+\nu_{e}\left(\bar{\nu}_{e}\right)+\bar{\nu}_{\mu}\left(\nu_{\mu}\right),
\end{aligned}
$$

with a similar chain for charged kaons. In the low-energy limit where all particles decay we therefore expect

$$
\nu_{\mu}+\bar{\nu}_{\mu} \approx 2\left(\nu_{e}+\bar{\nu}_{e}\right)
$$

At high energies the muon decay path becomes larger than the thickness of the atmosphere. In the high-energy limit muon decay no longer contributes and the main contribution to the flux of electron neutrinos is from decay of neutral kaons. The ratio of muon neutrinos to electron neutrinos is larger than 2. At very low energies the ratio of neutrino flavors approaches 1 . This again is a consequence of kinematics: the Michel spectrum for neutrinos from muon decay, when transformed into the laboratory, peaks at $E_{\nu}=0$. Thus the very-low-energy neutrinos come preferentially from muon decay, giving a ratio near one [23].

In 1985, two experiments in deep mines, one in the United States called IMB [24] and one in Japan called Kamiokande [25], observed a deficit in the number of muon neutrinos created in the atmosphere with respect to the number of electron neutrinos. This deficit became known as the "Atmospheric Neutrino Anomaly". Since then, several experiments measured the ratio of muon-like neutrino events to electron-like neutrino events, divided by their respective Monte Carlo simulation value:

$$
R^{\prime}=\frac{\left(\nu_{\mu} / \nu_{e}\right)_{d a t a}}{\left(\nu_{\mu} / \nu_{e}\right)_{M C}}
$$

Their results are summarized in Fig.1.4. The Kamiokande, IMB and Super-Kamiokande experiments are based on the water Cerenkov technique, while FREJUS, NUSEX, and 
SOUDAN have ionisation-based tracking detectors. The ratios are consistently below 1 except for FREJUS and NUSEX which had large error bars.

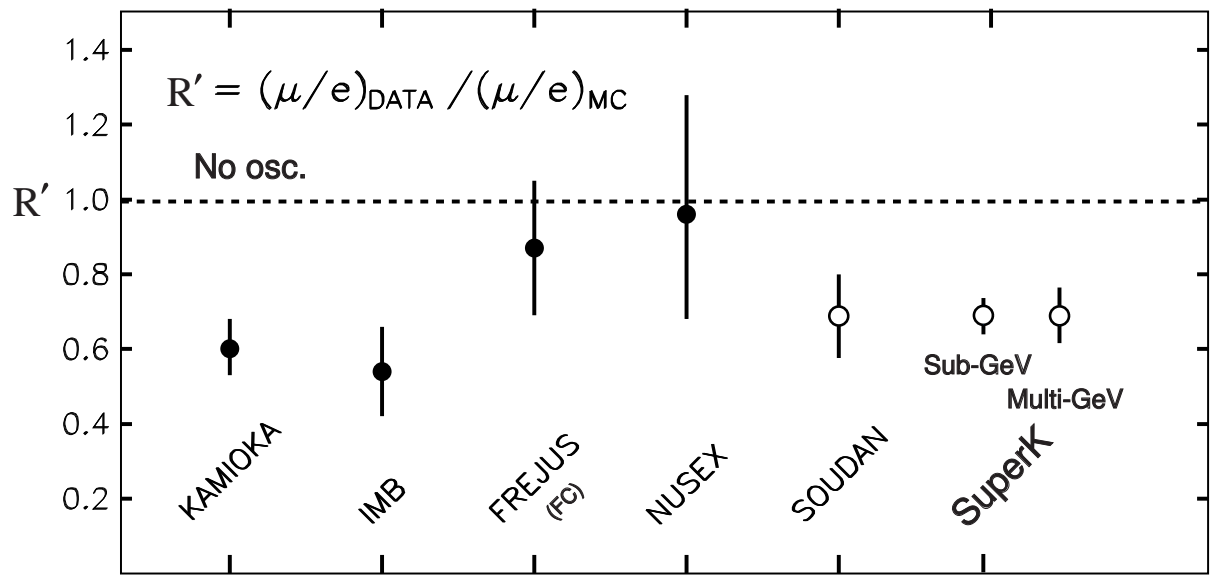

Figure 1.4: Ratio of muon-like neutrino events to electron-like neutrino events, divided by their respective Monte Carlo simulation value. Plot taken from [49].

In 1996, Super-Kamiokande (Super-K) was completed and began taking data. Super-K is a 50 kiloton water Cerenkov detector located at a depth of 2700 meters water equivalent in the Kamioka Mozumi mine in Japan. It supersedes its predecessors (IMB and Kamiokande) both in size and resolution and began detecting atmospheric neutrinos at much higher rates. In 1998, after analyzing more than 500 days of data, the experimentalists at Super-K announced that the atmospheric neutrino anomaly was not a statistical aberration and is consistent with two-flavor neutrino oscillations [50].

Super-K measured the zenith angle distributions of $\mu$-like and $e$-like events. Fig.1.5 shows the relation between zenith angle and the distance traveled by atmospheric neutrinos. Fig.1.6 shows the zenith angle distribution of $\mu$-like and $e$-like events for sub-GeV and multi-GeV data sets. Upward-going particles have $\cos \Theta<0$ and downward-going particles have $\cos \Theta>0$. The conclusion is that the deficit in muon neutrinos is mostly due to the upward traveling ones. Oscillations easily explain this: 
the muon neutrinos raining down on the mine do not have sufficient time to oscillate while those traveling through the Earth do. Detailed Super-K atmospheric neutrino data are very well described by the hypothesis that the oscillation is dominated by $\nu_{\mu} \rightarrow \nu_{\tau}$ conversion, and that it is a quasi-two-neutrino oscillation with a splitting $\Delta m_{\mathrm{atm}}^{2}$ and a mixing angle $\theta_{\mathrm{atm}}$ that, at $90 \% \mathrm{CL}$, are in the ranges [53]

$$
\sin ^{2} 2 \theta_{\mathrm{atm}}>0.92 \text { and } 1.5 \times 10^{-3}<\Delta m_{\mathrm{atm}}^{2}<3.4 \times 10^{-3} \mathrm{eV}^{2} .
$$

In the three-flavor neutrino mixing, $\theta_{\text {atm }} \approx \theta_{23}$ and $\Delta m_{\mathrm{atm}}^{2} \approx\left|\Delta m_{32}^{2}\right|$. For $\theta_{23}$ there is an ambiguity corresponding to $\theta_{23} \leftrightarrow \pi / 2-\theta_{23}$. Matter effects in future long-baseline experiments will resolve this. The often used parameter $\sin ^{2}\left(2 \theta_{23}\right)$ is blind to this ambiguity.

In 2004, the Super-K collaboration presented a new analysis of their data where they used a selected sample of events with good resolution in $L / E$ [54]. A dip in the $L / E$ distribution was observed around $L / E=500 \mathrm{~km} / \mathrm{GeV}$, as shown in Fig.(1.7). This provided the first direct evidence that the neutrino survival probability obeys the sinusoidal function as predicted by neutrino flavor oscillations. The 90\% C.L. allowed parameter region was obtained as

$$
\sin ^{2} 2 \theta_{\mathrm{atm}}>0.90 \text { and } 1.9 \times 10^{-3}<\Delta m_{\text {atm }}^{2}<3.0 \times 10^{-3} \mathrm{eV}^{2} .
$$

This result is consistent with that of the oscillation analysis using zenith angle distributions [53].

The oscillation interpretation of the atmospheric neutrino data has received support from the KEK to Kamioka $(\mathrm{K} 2 \mathrm{~K})$ long-baseline experiment. This experiment produces a beam of muon neutrinos with mean energy $\sim 1.3 \mathrm{GeV}$ at the KEK accelerator laboratory. These neutrinos are aimed at the Super-K detector in Kamioka, 250 


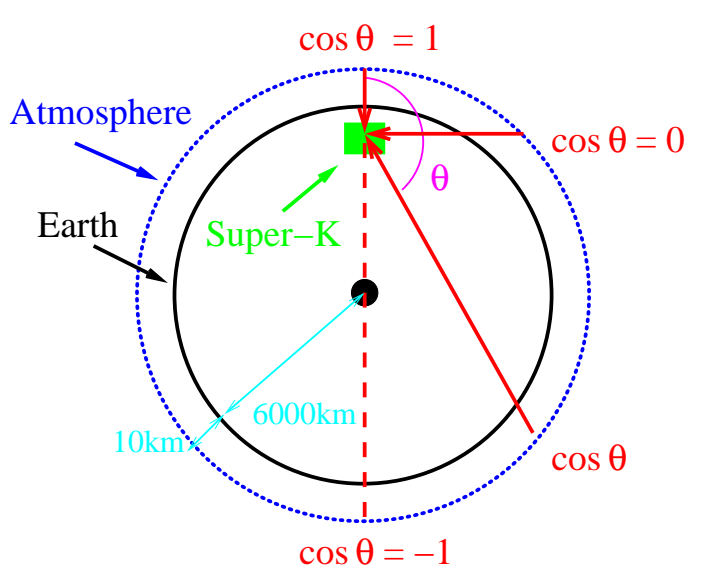

Figure 1.5: A sketch showing the relation between zenith angle and the distance traveled by atmospheric neutrinos (plot taken from [51]). $\cos \theta<0$ represents upward-going particles and $\cos \theta>0$ represents downward-going particles. The flight path of atmosphere neutrinos from production to detector extends from $\sim 10$ $\mathrm{km}$ to $12,000 \mathrm{~km}$.

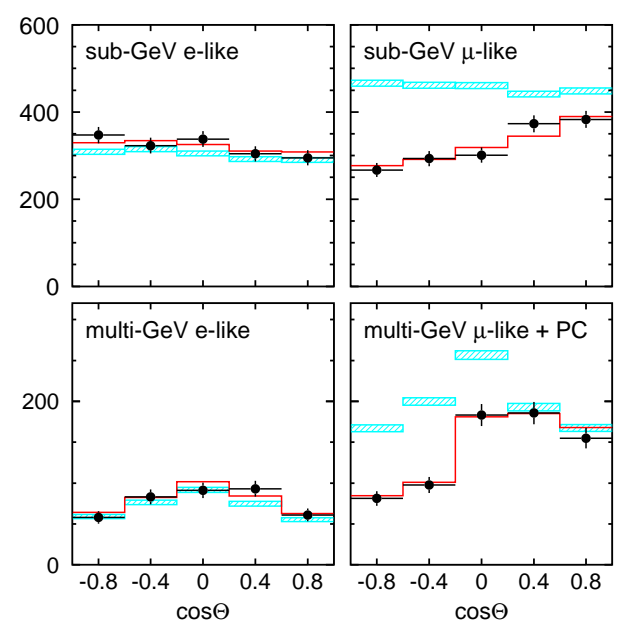

Figure 1.6: Zenith angle distributions of $\mu$-like and $e$-like events for sub-GeV and multi-GeV data sets. Upward-going particles have $\cos \Theta<0$ and downward-going particles have $\cos \Theta>0$. The bars show the MC no-oscillation prediction with statistical errors, and the line shows the bestfit expectation for $\nu_{\mu} \rightarrow \nu_{\tau}$ oscillations. Plots taken from [52]. 


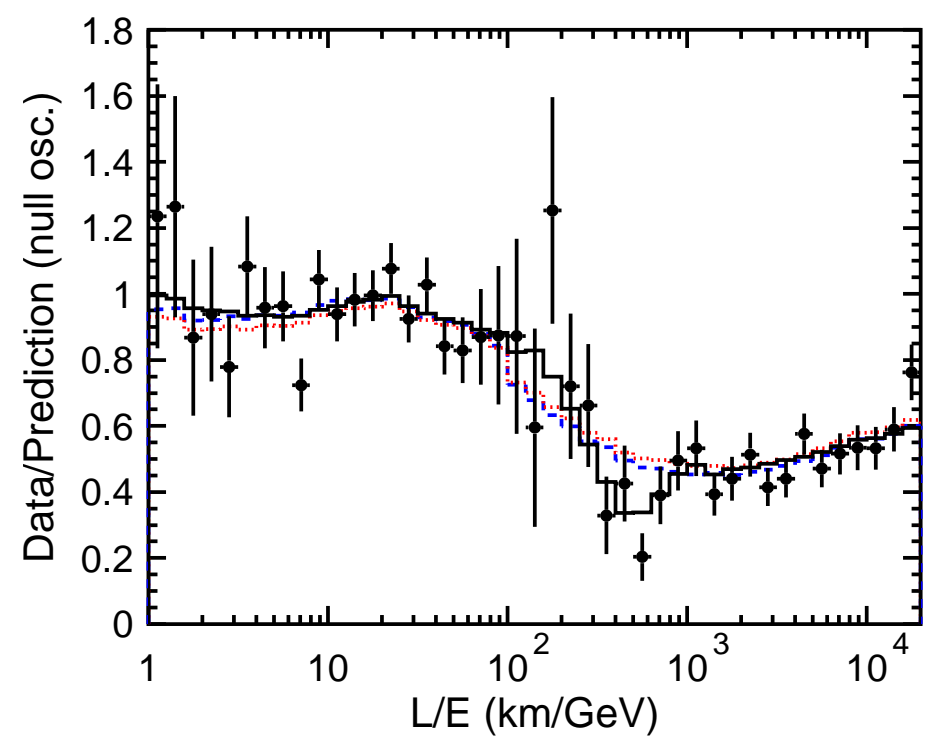

Figure 1.7: Ratio of the data to the $\mathrm{MC}$ events without neutrino oscillation (points) as a function of the reconstructed $L / E$ together with the best-fit expectation for 2flavor $\nu_{\mu} \rightarrow \nu_{\tau}$ oscillations (solid line). Also shown are the best-fit expectation for neutrino decay (dashed line) and neutrino decoherence (dotted line). Plot taken from [54]. 
$\mathrm{km}$ away. The K2K experiment reported that after achieving $8.9 \times 10^{19}$ protons-ontarget (about four and a half years) the expected number of events occurring in the fiducial volume of Super-K detector is $151_{-10}^{+12}$ (syst.). However, only 107 events were observed [55]. In addition, the spectrum of $\nu_{\mu}$ events observed in Super-K detector was distorted relative to the no-oscillation spectrum. The anomalously small number of events and spectral distortion seen by $\mathrm{K} 2 \mathrm{~K}$ experiment are consistent with a neutrino oscillation interpretation, with parameters $\Delta m_{\text {atm }}^{2}$ and $\theta_{\text {atm }}$ compatible with those that fit the atmospheric neutrino data.

The MINOS (Main Injector Neutrino Oscillation Search) is another long-baseline neutrino experiment similar in concept to K2K. This experiment uses a high-intensity muon neutrino beam generated at the Fermi National Accelerator Laboratory (Fermilab). The neutrino beam with maximal flux at around $3 \mathrm{GeV}$ is directed toward the Soudan Mine $735 \mathrm{~km}$ away in northern Minnesota. The MINOS experiment consists of two detectors, a 0.98 kton Near Detector (ND) at Fermilab and a 54 kton Far Detector (FD) at Soudan Mine. Compared to K2K, the MINOS experiment uses a three times longer distance, and the intensity and the energy of the MINOS neutrino beam are higher than the K2K beam. The MINOS experiment reported that based on a two-year exposure to the neutrinos from the Fermilab accelerator $\left(3.21 \times 10^{20}\right.$ protons-on-target), the expected number of events occurring in the fiducial volume of FD is $1065 \pm 60$ (syst.). However, only 848 events were observed. By fitting the spectrum of $\nu_{\mu}$ events in FD with the neutrino oscillation hypothesis, the MINOS ex-

periment achieved the most precise measurement of the mass splitting $\Delta m_{\mathrm{atm}}^{2}$. The MINOS data also disfavor alternative explanations for the disappearance of neutrinos in flight, such as neutrino decays into lighter particles or quantum decoherence of neutrinos, at the 3.7 and 5.7 standard deviation levels, respectively. Details of the MINOS experiment will be discussed in the next chapter. The latest MINOS results on the 23 Sector parameters are shown in Fig.1.8 along with the older results from 
the super-K atmospheric measurements and the K2K experiment. The oscillations in this sector are consistent with being maximal (i.e. $\theta_{23}=45^{\circ}$ ), but the precision with which $\theta_{23}$ is known is not great. It is an interesting open question how close to maximal $\theta_{23}$ really is.

The recent MINOS results shown in Fig.1.8 are still statistics limited and, as MINOS continues to take data over the next few years, it will continue to improve the constraints on $\theta_{23}$ and $\Delta m_{32}^{2}$. The upcoming experiments $\mathrm{T} 2 \mathrm{~K}$ and $\mathrm{NO} \nu \mathrm{A}$ are designed to probe the 13 Sector and are described more fully in the later sections, but they will also be able to make very precise measurements of the 23 Sector and probe the issue of how close $\theta_{23}$ comes to being maximal.

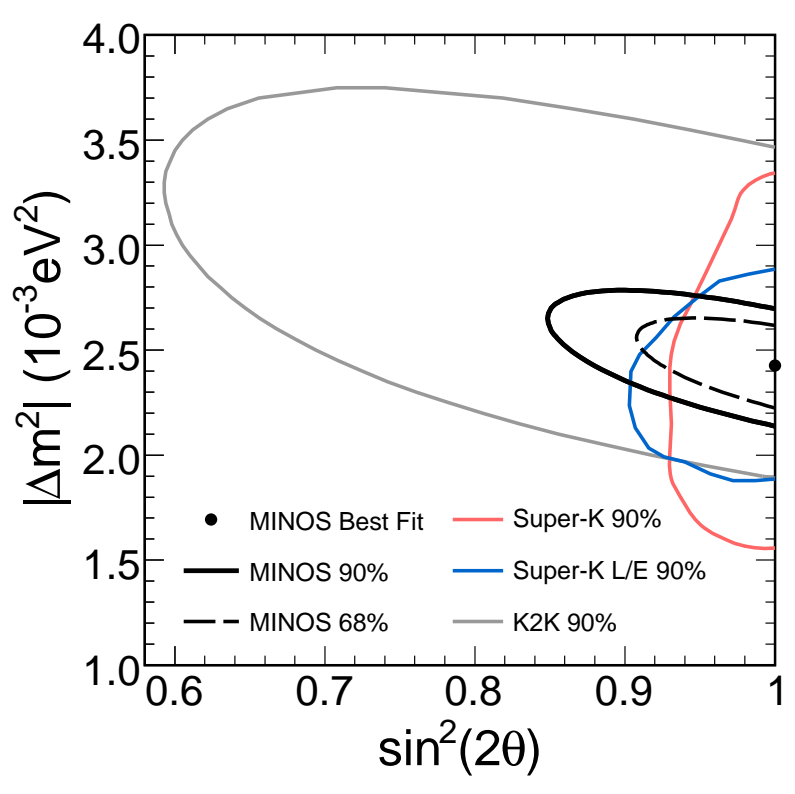

Figure 1.8: The new MINOS best fit point (black point) and the $68 \%$ and $90 \%$ CL contours. Overlaid are the 90\% CL contours from the Super-K zenith angle [53] and $L / E[54]$ analyses, and that from the K2K experiment [56]. Plot taken from [59].

If the $\nu_{\mu}$ 's are oscillating away in experiments with $\mathrm{GeV}$ energies and baselines 
hundreds of kilometers long then they must be oscillating into tau neutrinos if the scheme of mixings and masses described earlier is correct. It would be a powerful test of the scheme to look for these $\nu_{\tau}$ 's and the OPERA experiment [60] is designed to do just that. OPERA is a hybrid emulsion and tracking detector that has recently started taking data in Gran Sasso. It detects neutrinos from the CNGS beam created at CERN. The baseline is $732 \mathrm{~km}$ and the mean neutrino energy is $17 \mathrm{GeV}$.

\subsubsection{The 13 Sector}

The 13 Sector comprises the mixing angle $\theta_{13}$ and the phase $\delta$ which, if different from 0 or $\pi$, would induce $\mathrm{CP}$ violation into the scheme of neutrino oscillations. Note the squared mass difference $\Delta m_{31}^{2}$ between $\nu_{3}$ and $\nu_{1}$ is not an independent parameter and $\Delta m_{31}^{2}=m_{3}^{2}-m_{1}^{2}=\Delta m_{32}^{2}+\Delta m_{21}^{2} \simeq \Delta m_{32}^{2}$ since $\left|\Delta m_{21}^{2}\right| \ll\left|\Delta m_{32}^{2}\right|$. Another issue that is often discussed in the context of the 13 Sector is the mass hierarchy which is the question of whether the masses are ordered with the almost degenerate doublet $\nu_{1}$ and $\nu_{2}$ higher or lower than the $\nu_{3}$ mass. This is so far the least understood Sector. There are no constraints of any significance on $\delta$ and the mass hierarchy is unknown. We do have constraints on $\theta_{13}$, however, provided by experiments measuring electron anti-neutrino disappearance at baselines roughly 1 $\mathrm{km}$ from the reactor source. Besides the disappearance of reactor anti-neutrinos, the 13 Sector can also be probed by looking for $\nu_{\mu} \rightarrow \nu_{e}$ oscillations using baselines and energies sensitive to the parameter $\Delta m_{31}^{2}$.

Sec.1.4.1 described how the KamLAND experiment used reactor anti-neutrinos and a baseline of order $200 \mathrm{~km}$ to measure parameters of the 12 Sector. In this configuration the $L / E$ is matched to $\Delta m_{12}^{2}$. By instead measuring the disappearance of reactor anti-neutrinos at a baseline $L$ of $\sim 1 \mathrm{~km}$ one can match the $L / E$ to $\Delta m_{31}^{2}$ and be sensitive to the parameters of the 13 Sector. The expression for the electron 
anti-neutrino survival probability is then

$$
P\left(\bar{\nu}_{e} \rightarrow \bar{\nu}_{e}\right) \approx 1-\sin ^{2} 2 \theta_{13} \sin ^{2}\left(1.27 \Delta m_{31}^{2} L / E_{\nu}\right) .
$$

The best measurement to date is the Chooz experiment that operated in France in the 1990's [61]. The Chooz experiment detected electron anti-neutrinos with a liquid scintillation calorimeter located $1.05 \mathrm{~km}$ from the reactor core. They found no evidence for neutrino oscillations in the $\bar{\nu}_{e}$ disappearance mode. For $\Delta m^{2}$ equal to the current MINOS best fit value $2.4 \times 10^{-3} \mathrm{eV}^{2}$, the constraint on $\theta_{13}$ from Chooz is $\sin ^{2} 2 \theta_{13} \lesssim 0.15$ at $90 \%$ CL. Another measurement of the 13 Sector is the Palo Verde experiment operated in Arizona, USA [62]. This experiment measured the anti-neutrino flux and spectrum at a distance of about $800 \mathrm{~m}$ from the three reactors of the Palo Verde Nuclear Generating Station using a segmented gadolinium-loaded scintillation detector. They excluded at $90 \%$ CL $\bar{\nu}_{e} \rightarrow \bar{\nu}_{x}$ oscillations. They posed a constraint on the parameter $\theta_{13}$ : at the current MINOS best fit point, $\sin ^{2} 2 \theta_{13} \lesssim 0.24$ at 90\% CL. Fig.1.9 shows the 90\% CL exclusion regions for the Chooz experiment and the Palo Verde experiment.

It has been reported that the solar and KamLAND data provide a non-trivial constraint on $\theta_{13}$ especially for lower values of $\Delta m_{\text {atm }}^{2}$, see e.g., Refs. [63, 64, 65]. Fig.1.10 shows the current constraints on the 13 Sector mixing parameters from global data. The Chooz bound on $\sin ^{2} \theta_{13}$ gets quickly weak when $\Delta m_{\text {atm }}^{2}$ decreases. Such loosening in sensitivity is prevented to some extent by solar neutrino and KamLAND data. The constraint on $\theta_{13}$ from global data is $\sin ^{2} 2 \theta_{13} \lesssim 0.11$ [66].

A new generation of reactor experiments is being constructed in the hope of improving on the Chooz measurement and further constraining the value of $\theta_{13}$, either limiting it to be even closer to zero or measuring a no-zero value for it. These new experiments hope to be sensitive to a value of $\sin ^{2} 2 \theta_{13}$ as small as 0.01 . To do this 


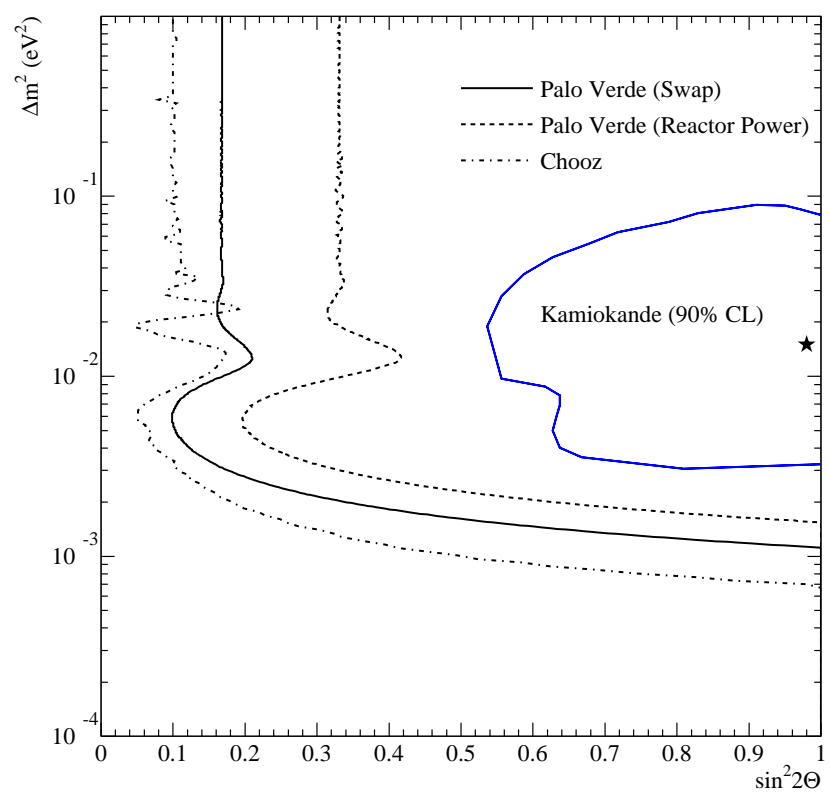

Figure 1.9: The 90\% CL exclusion regions for the Chooz experiment and the Palo Verde experiment. "Swap" and "Reactor Power" represent two analysis methods used in the Palo Verde experiment. Plot taken from [62]. 


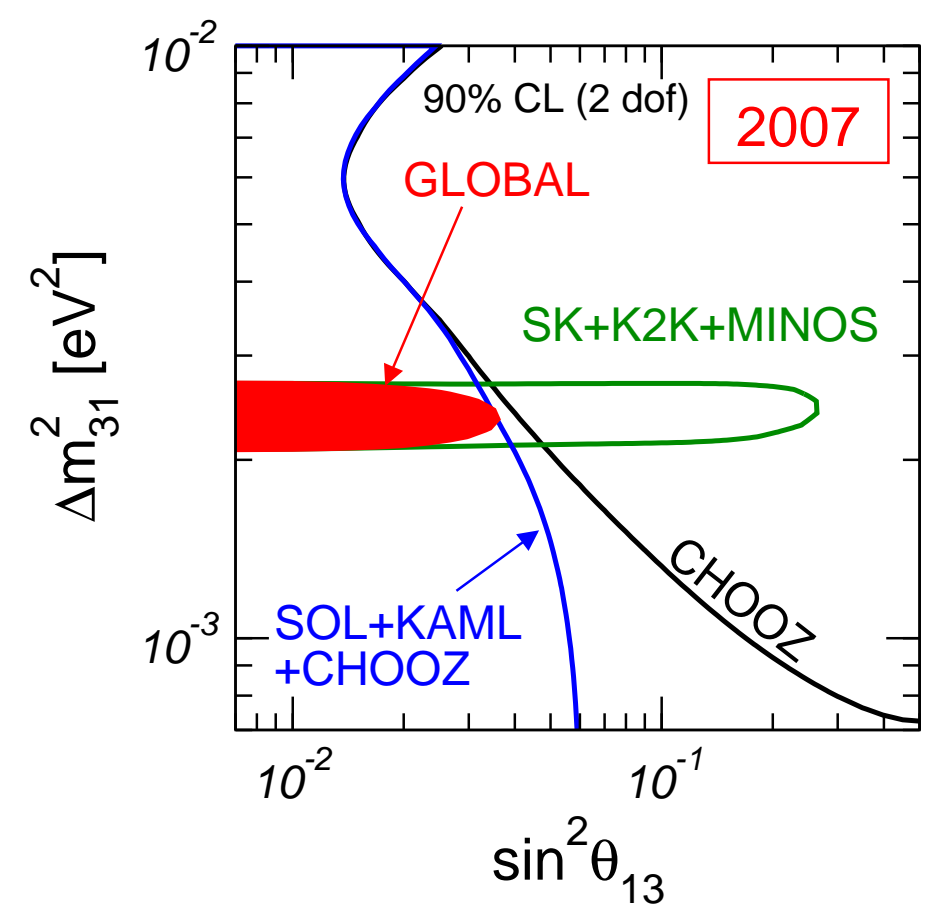

Figure 1.10: Current state of knowledge of the 13 Sector. Plot taken from [66].

they are making several upgrades to the Chooz approach. Most importantly they are using multiple detectors to cancel systematics. These detectors will be larger, be located at very high flux reactors, and be exposed to the beam for longer. They will be underground to reduce the effect of cosmics and be thoroughly calibrated. The main contenders in this next round are Double Chooz [67], located at the same site as the original Chooz experiment, the Daya Bay experiment located in China [68], the Angra experiment located in Brazil [69], and the RENO experiment located in South Korea [70].

The second way to probe the 13 Sector is by looking for the sub-dominant $\nu_{\mu} \rightarrow \nu_{e}$ oscillations at values of $L / E$ matched to $\Delta m_{31}^{2}$. This is the focus of my thesis. If we ignore the matter effect, solar terms, and $\mathrm{CP}$ violating phase, the oscillation 
probability is

$$
P\left(\nu_{\mu} \rightarrow \nu_{e}\right) \approx \sin ^{2} \theta_{23} \sin ^{2} 2 \theta_{13} \sin ^{2}\left(1.27 \Delta m_{31}^{2} L / E_{\nu}\right)
$$

Unlike the $\bar{\nu}_{e}$ survival probability Eq.(1.107), the $\nu_{e}$ appearance probability Eq.(1.108) depends not only on the parameter $\theta_{13}$, but also on the parameter $\theta_{23}$. Thus measuring $\theta_{13}$ by search for $\nu_{\mu} \rightarrow \nu_{e}$ appearance relies on a good understanding of the mixing angle $\theta_{23}$. The MINOS experiment is designed to probe the 23 Sector by measuring the disappearance of $\nu_{\mu}$ events. The recent results of this measurement are shown in Fig.1.8. MINOS will also be able to improve the current best limit on the neutrino mixing angle $\theta_{13}$ by searching for an electron neutrino appearance signal in the Far Detector from the $\nu_{\mu}$ neutrino beam. MINOS may make the first measurement of $\theta_{13}$ with improved proton intensity if $\theta_{13}$ is sufficiently large. More details of the MINOS $\nu_{e}$ appearance measurement will be discussed in the later chapters.

A second generation of long baseline accelerator neutrino oscillation experiments has been proposed. They hope to extend the sensitivity for $\nu_{e}$ appearance roughly a factor of 10-20 beyond the CHOOZ limit. The matter effects induced by the long baseline increase the potential to search for $\mathrm{CP}$-violating phase $\delta$ and resolve mass hierarchy in the neutrino sector. These experiments will make use of an off-axis beam. By placing the detector slightly off the neutrino beam axis (e.g. 15mrad), the detector will see a narrow band beam peaked at low energy (e.g. 2GeV). Because the backgrounds (intrinsic $\nu_{e}$ and neutral current events) in the $\nu_{e}$ appearance search are much broader in energy than the signal, a narrow beam will allow a much better signal to background ratio than for the MINOS measurement. Other highlights of these experiments include improved beam intensity, large detectors optimized for $\nu_{e}$ detection, and using two detectors to cancel systematics. There are two long baseline experiments under construction that will probe the 13 Sector, first by searching for a 
non-zero $\theta_{13}$ and if one is found then determining the mass hierarchy and searching for $\mathrm{CP}$ violation. The two experiments are $\mathrm{NO} \nu \mathrm{A}[71]$ and $\mathrm{T} 2 \mathrm{~K}[72]$. $\mathrm{NO} \nu \mathrm{A}$ wil use the beamline currently used by MINOS, with a new detector being built $810 \mathrm{~km}$ away in far northern Minnesota. T2K will use the existing Super-K detector and the beam will be sent from the JPARC accelerator lab currently under construction about 250 $\mathrm{km}$ away.

\subsubsection{The LSND Result and MiniBooNE}

The Liquid Scintillator Neutrino Detector (LSND) experiment [73] operated at Los Alamos National Lab in the 1990's and produced evidence for $\bar{\nu}_{\mu} \rightarrow \bar{\nu}_{e}$ oscillations at the $\Delta m^{2} \sim 1 \mathrm{eV}^{2}$ scale. This $\Delta m^{2}$ scale is incompatible with those of the solar and atmospheric oscillations, and so requires there be more than 3 neutrinos if all three are to be interpreted as evidence of neutrino oscillation.

The Mini-Booster Neutrino Experiment (MiniBooNE) was built to test the oscillation interpretation of the LSND result. The detector is located $540 \mathrm{~m}$ from the target and comprises a spherical tank filled with 800 tons of pure mineral oil $\left(\mathrm{CH}_{2}\right)$. This experiment can distinguish electrons from other particles (in particular $\nu$ 's and $\pi^{0}$ 's) and so test for $\nu_{\mu} \rightarrow \nu_{e}$ oscillations. In April 2007 the experiment released its first results [74]. The experiment found no evidence of neutrino oscillations in its analysis region above a neutrino energy of $475 \mathrm{MeV}$, though there was a excess of events found below this energy and this is currently under investigation. The exclusion plot that summarizes results from this measurement is shown in Fig.1.11. The MiniBooNE and LSND results are only compatible at the $2 \%$ level if both are interpreted in the framework of two flavor neutrino oscillations. MiniBooNE is currently taking data in anti-neutrino mode (where the horn focuses negative particles) and intends to make a measurement of $\bar{\nu}_{e}$ appearance to more fully check the LSND result. 


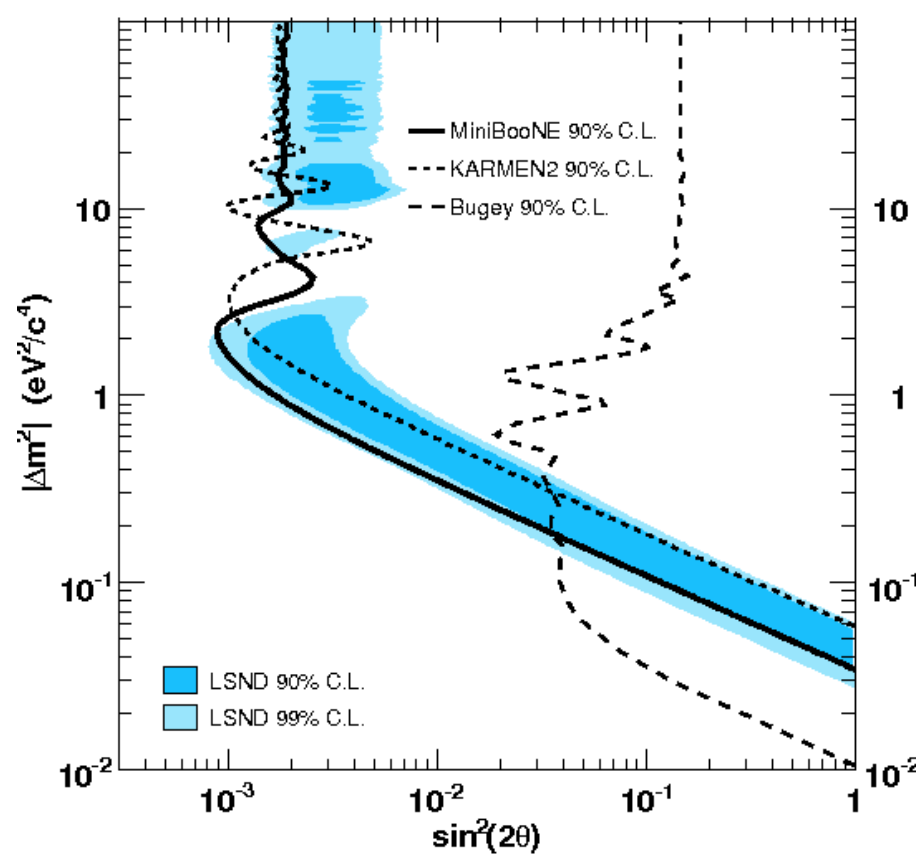

Figure 1.11: The region of oscillation parameter psace excluded at 90\% CL by the MiniBooNE result. Also shown are the regions allowed by the LSND result at $90 \%$ CL and 95\% CL, and the 90\% exclusion contours of the KARMEN2 [75] and Bugey [76] experiments. Plot taken from [74]. 


\subsubsection{Neutrinoless Double $\beta$ Decay}

In several nuclei with an even number of neutrons and an even number of protons the extra binding energy produced by the pairing leaves ordinary $\beta$ decay energetically forbidden. In such nuclei double beta decay, where two electrons are emitted, is left as the only viable decay mode. Two neutrino double beta $(2 \nu \beta \beta)$ decay has by now been observed in a number of nuclei, but neutrinoless double beta $(0 \nu \beta \beta)$ decay has yet to be convincingly seen. If observed, $0 \nu \beta \beta$ decay would imply that neutrinos are Majorana particles. It is expected that the process will be dominated by the diagram shown in Fig.1.12. In this diagram, one or another of the neutrino mass eigenstates $\nu_{i}$ is exchanged between two virtual $W$ bosons to create the outgoing electrons. The $0 \nu \beta \beta$ amplitude is then a coherent sum over the contributions of the different $\nu_{i}$. The

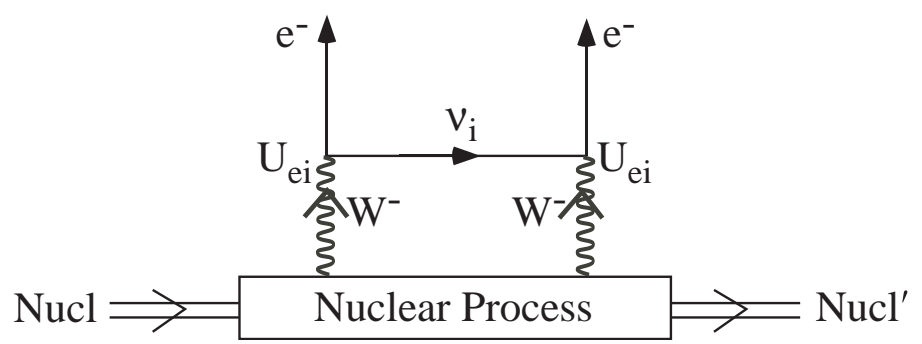

Figure 1.12: Neutrinoless double beta decay. Plot taken from [33].

rate for the process is given by

$$
\begin{aligned}
\Gamma_{0 \nu} & =G_{0 \nu}\left|M_{0 \nu}\right|^{2} m_{\beta \beta}^{2} \\
m_{\beta \beta} & =\left|\sum_{i} m_{i} U_{e i}^{2}\right|
\end{aligned}
$$

where $G_{0 \nu}$ is a readily calculable phase space factor and $M_{0 \nu}$ is the, not so readily calculable, matrix element for the process. $U_{e i}$ and $m_{i}$ are the mixing matrix elements and neutrino masses and $\nu_{e}=\sum_{i} U_{e i} \nu_{i}$. The signature for the $0 \nu \beta \beta$ process is a peak in the measured energy of the pair of electrons at the $Q$ value, where $Q$ is 
generally defined as (initial energy) - (mass of all particles in the final state except the neutrinos).

\subsubsection{Kinematic tests for $\nu$ masses}

There are tests on processes which are allowed in the Standard model even with $m_{\nu}=0$. We can take any such known process involving neutrinos in the final state and then calculate the rate as a function of neutrino mass. We then try to see whether the observed rate differs significantly from the calculated rate with $m_{\nu}=0$. Here are some exaples.

- Nuclear $\beta$-decay: One can look at the beta spectrum in $(Z, A) \rightarrow(Z+1, A)+$ $e^{-}+\bar{\nu}_{e}$ (Kurie plot) or corresponding positron decay. The shape of the curve can be calculated assuming $m_{\nu_{e}}=0$. If, however the mass is not zero, the observed count will fall short of the calculated one as the electron energy approaches the total decay energy $E_{0}=M_{i}-M_{f}$, where $M_{i}$ and $M_{f}$ are the masses of the initial atom and the final ion. The fraction of decays when the electron energy is close to $E_{0}$ becomes rapidly smaller for beta decays with higher $Q$ values. It is therefor, imperative to select a candidate with low $Q$. A good candidate should also have a short lifetime, which means atoms decay more rapidly, making more data available. Tritium is a perfect source by both of these measures: it has a reasonably short lifetime (12.4 years) and has very low $\mathrm{Q}$ value $(18.6 \mathrm{keV})$. Additionally, its molecular structure is simple enough that the energy spectrum of the decay electrons can be calculated with confidence.

- Pion decay: One can look for the muon energy in $\pi^{+} \rightarrow \mu^{+} \nu_{\mu}$ (or its charge conjugate decay). Obviously this energy depends on the $\nu_{\mu}$ mass.

- Tau decay: There are various decay modes of the tau. One can use the kinematics of the final state to find the mass of the $\nu_{\tau}$. 
Direct Kinematic tests $[77,78,79]$ have yielded the results

$$
\begin{aligned}
m_{\nu_{e}}<2.2 \mathrm{eV} \quad\left(95 \% \mathrm{CL}, \text { from }{ }^{3} \mathrm{H} \rightarrow{ }^{3} \mathrm{He}+e^{-}+\bar{\nu}_{e}\right), \\
m_{\nu_{\mu}}<170 \mathrm{keV} \quad\left(90 \% \mathrm{CL}, \text { from } \pi^{+} \rightarrow \mu^{+}+\nu_{\mu}\right), \\
m_{\nu_{\tau}}<15.5 \mathrm{MeV} \quad\left(95 \% \mathrm{CL}, \text { from } \tau \rightarrow 5 \pi+\nu_{\tau}\right) .
\end{aligned}
$$

Strictly speaking, these experiments do not measure $m_{\nu_{e}}, m_{\nu_{\mu}}$, or $m_{\nu_{\tau}}$, which are not the neutrino mass eigenstates. Instead they measure some mixture of $m_{1} \equiv m\left(\nu_{1}\right)$, $m_{2}$, and $m_{3}$.

\subsubsection{Summary}

Table 1.2 summarizes the current state of knowledge of the neutrino parameters.

\begin{tabular}{ccc}
\hline Parameter & Best-fit value & Range \\
\hline$\Delta m_{21}^{2}$ & $8.0 \times 10^{-5} \mathrm{eV}^{2}$ & $(7.7-8.4) \times 10^{-5} \mathrm{eV}^{2}( \pm 1 \sigma)$ \\
$\left|\Delta m_{31}^{2}\right|$ & $2.43 \times 10^{-3} \mathrm{eV}^{2}$ & $(2.30-2.56) \times 10^{-3} \mathrm{eV}^{2}( \pm 1 \sigma)$ \\
$\theta_{12}$ & $33.9^{\circ}$ & $32.3^{\circ}-35.5^{\circ}( \pm 1 \sigma)$ \\
$\theta_{13}$ & unknown & $0^{\circ}-11.4^{\circ}(90 \%$ C.L. $)$ \\
$\theta_{23}$ & $45^{\circ}$ & $36.8^{\circ}-53.2^{\circ}(90 \%$ C.L. $)$ \\
$\delta_{\mathrm{CP}}$ & unknown & \\
$\mathrm{m}_{\text {lightest }}$ & unknown & $0-2.2 \mathrm{eV}(95 \%$ C.L. $)$ \\
Hierarchy & unknown & \\
Dirac or Majorana & unknown & \\
\hline
\end{tabular}

Table 1.2: The current state of knowledge of the neutrino parameters.

Ignoring the phases and assuming $\theta_{13}=0$, the "best-fit PMNS matrix" reads:

$$
U_{P M N S}^{B F}=\left[\begin{array}{ccc}
0.83 & 0.56 & 0 \\
-0.39 & 0.59 & 0.71 \\
0.39 & -0.59 & 0.71
\end{array}\right]
$$


The data can be well fitted by the tri-bimaximal mixing of the form:

$$
U_{T B}=\left[\begin{array}{ccc}
\sqrt{\frac{2}{3}} & \frac{1}{\sqrt{3}} & 0 \\
-\frac{1}{\sqrt{6}} & \frac{1}{\sqrt{3}} & \frac{1}{\sqrt{2}} \\
\frac{1}{\sqrt{6}} & -\frac{1}{\sqrt{3}} & \frac{1}{\sqrt{2}}
\end{array}\right] \approx\left[\begin{array}{ccc}
0.82 & 0.58 & 0 \\
-0.41 & 0.58 & 0.71 \\
0.41 & -0.58 & 0.71
\end{array}\right]
$$

The above tri-bimaximal mixing has $\theta_{12}=\sin ^{-1}(1 / \sqrt{3})=35.2^{\circ}, \theta_{23}=45^{\circ}$, and $\theta_{13}=0$. The tri-bimaximal form for the mixing matrix (up to a trivial sign rearrangement) was first proposed by Harrison, Perkins and Scott [83] and further studies by authors in [84]. Many theoretical efforts have been made to produce such a mixing pattern. 


\section{Chapter 2}

\section{The MINOS Experiment}

The Main Injector Neutrino Oscillation Search (MINOS) is a long-baseline neutrino oscillation experiment that performs precision measurements of the neutrino oscillation parameters in the "atmospheric neutrino" sector. The neutrinos are produced by the Neutrinos at the Main Injector (NuMI) facility at the Fermi National Accelerator Laboratory (Fermilab). MINOS measures neutrino interactions with a Near Detector at Fermilab and again $735 \mathrm{~km}$ downstream with a Far Detector in the Soudan Underground Laboratory in northern Minnesota. The two detectors are magnetized steel-scintillator tracking calorimeters. Comparison of the neutrino energy spectra and flavor composition of the beam at the two detectors will allow measurement of neutrino oscillation parameters. In this chapter, I will start with an introduction to the Fermilab accelerator. Then I will describe the neutrino production in the NuMI beamline and discuss the details of the MINOS detectors, which are steel-scintillator sampling calorimeters. In the end I will briefly discuss the physics reach of the MINOS experiment. 


\subsection{The Fermilab Accelerator}

The NuMI neutrino beam is created at Fermilab using $120 \mathrm{GeV}$ protons from the Main Injector. Fig.2.1 shows the schematic drawing of the Fermilab accelerators. The process of particle acceleration at Fermilab begins with a small bottle of hydrogen located in the electrostatic Preaccelerator. Fermilab's Preaccelerator is based on the Cockcroft-Walton design. It produces $\mathrm{H}^{-}$ion ${ }^{1}$ with an energy of $750 \mathrm{keV}$. The $\mathrm{H}^{-}$ ions travel through the Linac, the LINear ACcelerator. The Linac accelerates the ions to $400 \mathrm{MeV}$ and sends them to the Booster. The Booster takes $400 \mathrm{MeV} \mathrm{H}^{-}$ions from the Linac, strips the electrons off, accelerates the remaining protons to $8 \mathrm{GeV}$, and then sends them to the Main Injector. The Main Injector (MI) can accelerate the particles all the way up to $150 \mathrm{GeV}$. The NuMI beamline uses $120 \mathrm{GeV}$ protons from the Main Injector to produce a high intensity $\nu_{\mu}$ beam.

The Fermilab Booster is a rapid-cycling, $15 \mathrm{~Hz}$, alternating gradient synchrotron with a radius of 75.47 meters. It accelerates protons from a kinetic energy of $400 \mathrm{MeV}$ to $8 \mathrm{GeV}$, using $17 \mathrm{RF}$ cavities with frequency that slews from $37.8 \mathrm{MHz}$ at injection to $52.8 \mathrm{MHz}$ at extraction to match the MI frequency. Typically, the injection process lasts for ten Booster turns, resulting in a total average current of $420 \mathrm{~mA}$. The injected beam is a stream of bunches equally spaced at the linac RF frequency of $201.2 \mathrm{MHz}$. The Booster batch size is roughly $5 \times 10^{12}$ protons per batch.

The Main Injector is another synchrotron with a radius of 528.30 meters, and its acceleration cycle is $2.2 \mathrm{~s}$. It accepts $8 \mathrm{GeV}$ protons from Booster and accelerates protons to $120 \mathrm{GeV}$ or $150 \mathrm{GeV}$. Fig.2.2 shows the injection of the Booster batches in the Main Injector as a function of time. The total Main Injector cycle is composed of three parts: injection, acceleration, and resetting.

- Injection time is proportional to the number of Booster batches involved. In

\footnotetext{
${ }^{1}$ Hydrogen consists of a proton and an electron. The $\mathrm{H}^{-}$ion has an extra electron added.
} 


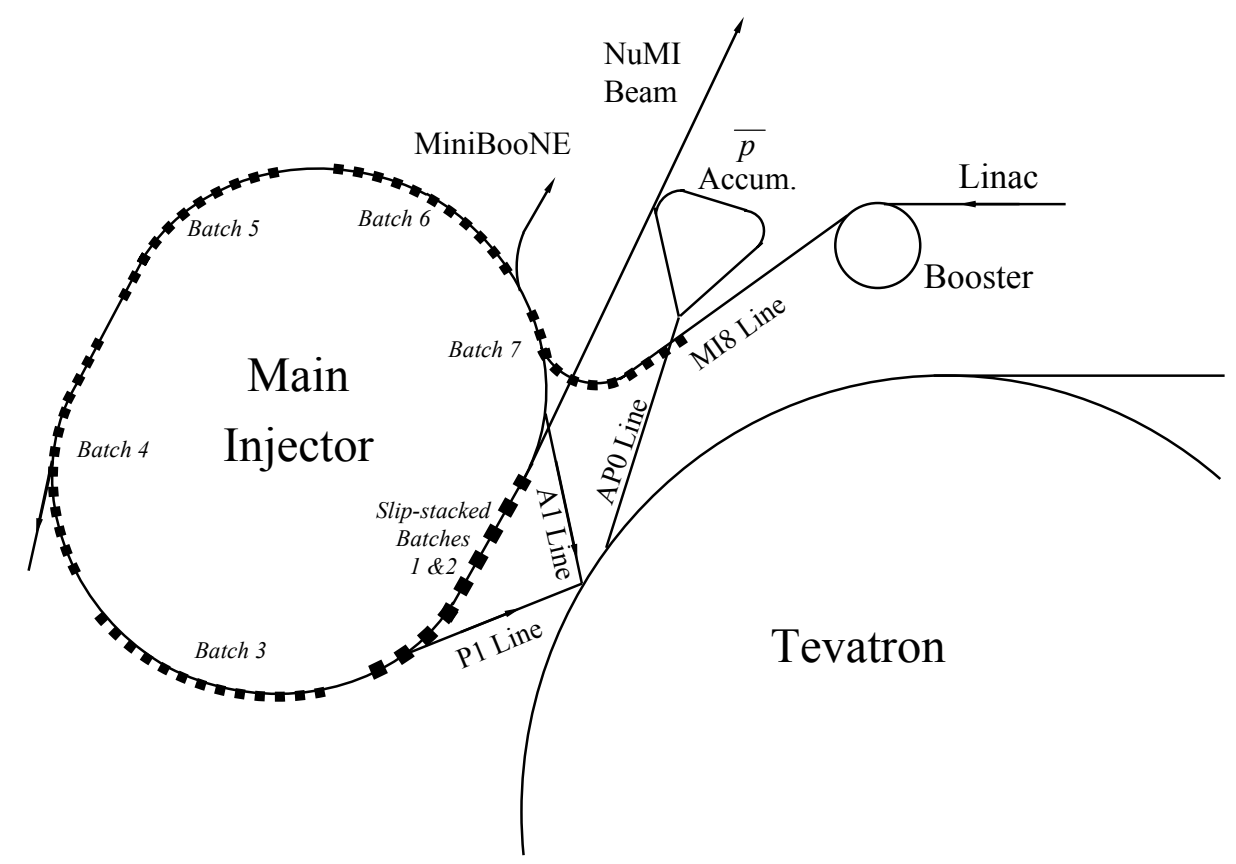

Figure 2.1: The Fermilab accelerators. Those used in generation of the NuMI beam are the Linac, Booster, and Main Injector. Plot taken from [85].

our highest intensity cycles, we presently have 11 Booster batches, coming at $15 \mathrm{~Hz}$ for a total of roughly $0.7 \mathrm{~s}$.

- Acceleration is a mostly fixed time. For MINOS it takes approximately $0.7 \mathrm{~s}$. At the end of acceleration there is extraction, but that takes a negligible period of time.

- After extraction, the Main Injector magnets must be ramped down to the field for $8 \mathrm{GeV}$.

Presently, the sum of the above processes takes $2.2 \mathrm{~s}$ for 11-batch injection. Most frequently, the Main Injector accepts $8 \mathrm{GeV}$ protons from Booster and accelerates protons to $120 \mathrm{GeV}$, and then sends them to the Pbar target, which yields $8 \mathrm{GeV}$ antiprotons, or to the NuMI target, which produces neutrinos for the MINOS experiment. Alternatively, it can accelerate protons to $150 \mathrm{GeV}$ and inject them into the 


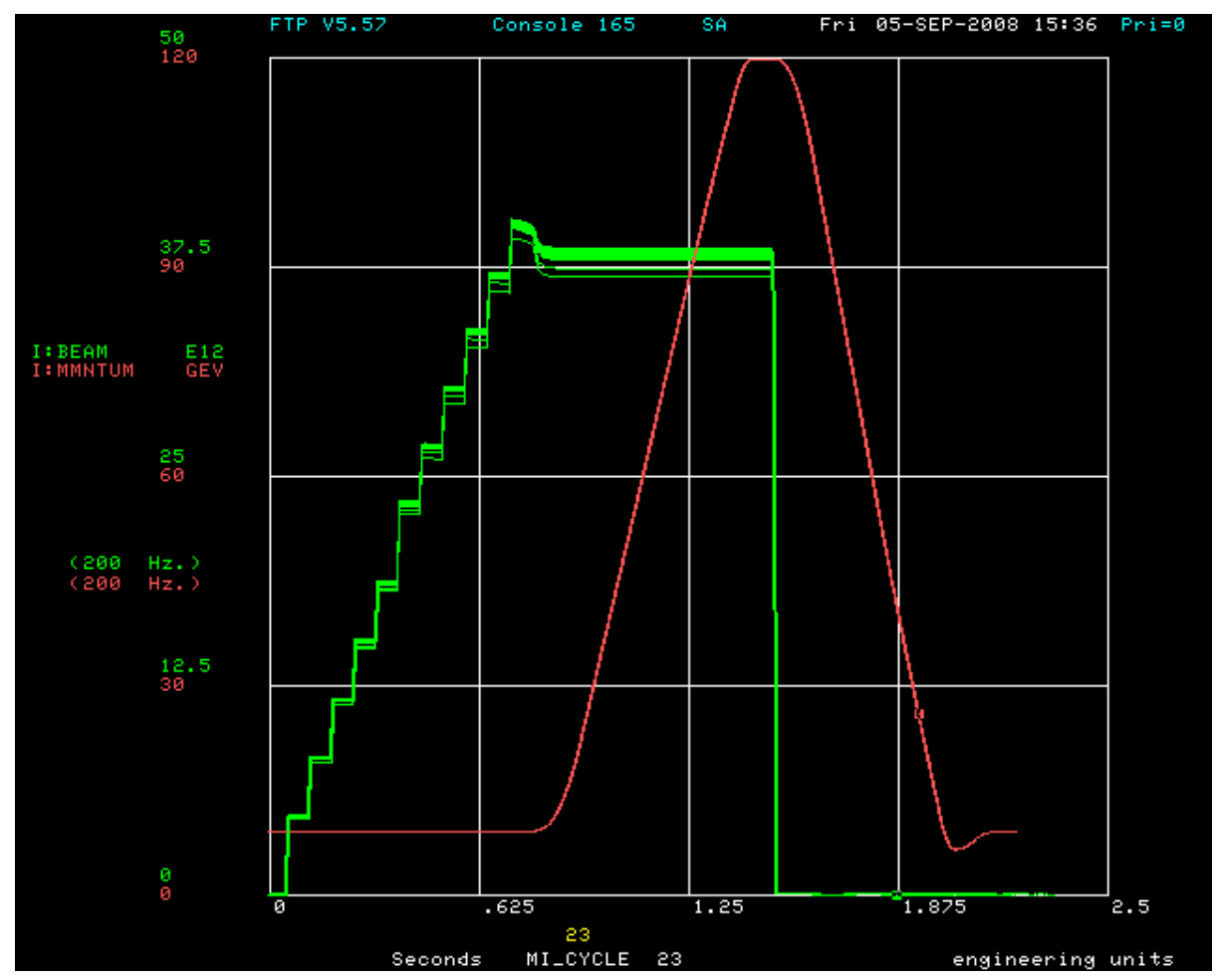

Figure 2.2: Injection of the Booster beam in the Main Injector as a function of time. Overlaid on top of each other at two traces: the MI charge (green) that increases 11 times in 11 separate injections from the Booster and the MI beam momentum (red) that is defined by the magnet currents, it ramps from 8 to $120 \mathrm{GeV} / c$, then ramps down to $8 \mathrm{GeV} / c$. 
Tevatron, where the beam is used for high energy particle physics experiments.

The main injector circumference is exactly 7 times the booster circumference, so there is room for 7 booster batches. However, one slot must remain empty to allow the injection kicker to ramp down. A fast single turn extraction kicker was required for the NuMI project to spill $120 \mathrm{GeV}$ protons onto a target. The extraction kicker has to rise in the $\sim 1.5 \mu \mathrm{s}$ abort gap of the Main Injector and then extract $1.6 \mu \mathrm{s}$ of beam (a single batch) for antiproton production and $8.0 \mu \mathrm{s}$ of beam ( 5 batches) to NuMI. This mode is referred to as "mixed mode". There is a second operational mode which is referred to as "NuMI only mode". In this mode, $9.6 \mu s$ (6 batches) of beam is extracted to NuMI [86].

A technique called "slip stacking" is utilized to increase the number of protons available for both the antiproton production and the NuMI neutrino production at Fermilab. This involves stacking two booster batches end to end but with slightly different momenta, into the Main Injector. The two batches have different periods of revolution and 'slip' relative to each other azimuthally and finally overlap. When they overlap they are captured using a single RF which is the average of the initial frequencies associated with the two batches [87].

Starting 2008, a "multi-batch slip stacking" mode becomes the standard operational mode. In this mode, five batches are loaded into the MI, and six more batches are loaded and slipped with the first five to make two batches for the antiproton production and nine for NuMI. This mode is referred to as " $2+9$ " mode. The NuMI beam intensity is greatly improved in this operational mode. A typical beam intensity is $3.1 \times 10^{13} \mathrm{POT} /$ spill for the "mixed mode" and $3.7 \times 10^{13} \mathrm{POT} /$ spill for the "NuMI only mode" with 8 booster turns. The corresponding beam power is $230 \mathrm{~kW}$ and 270 $\mathrm{kW}$, respectively. 


\subsection{The NuMI Neutrino Beam}

The Neutrinos at the Main Injector (NuMI) facility at Fermilab began operations in late 2004. NuMI produces a neutrino beam resulting from the decays of pion and kaon secondaries produced in the NuMI target. Protons of $120 \mathrm{GeV}$ are extracted from the MI accelerator and bent downward by $58 \mathrm{mrad}$ toward Soudan, MN, the site of the MINOS Far Detector. The global positioning system (GPS) defined the survey beam direction to within $12 \mathrm{~m}$ of the Far Detector. The primary beam is focused onto a rectangular graphite production target. The target dimensions are $6.4 \mathrm{~mm}$ in width, $15 \mathrm{~mm}$ in height and $940 \mathrm{~mm}$ in length (1.9 interaction lengths). The target is segmented longitudinally into 47 individual $2 \mathrm{~cm}$ length fins. The beam size at the target is $1.2-1.5 \mathrm{~mm}$. The target is water cooled via stainless steel tubes at the top and bottom of each fin. The particles produced in the target are focused (one sign only) by two magnetic horns. The focused beam of particles enters into a $675 \mathrm{~m}$ long, $2 \mathrm{~m}$ diameter steel pipe initially evacuated. This length is approximately the decay length of a $10 \mathrm{GeV}$ pion. Neutrinos are produced by decays of the secondaries, e.g. $\pi^{+}\left(K^{+}\right) \rightarrow \mu^{+} \nu_{\mu}$. The entrance to the decay pipe is sealed by a two-piece aluminumsteel window. The central (radius $<50 \mathrm{~cm}$ ) portion of the window is made of $1 \mathrm{~mm}$ thick aluminum and is strengthened by an outer (radius $>50 \mathrm{~cm}$ ) section made of $1.8 \mathrm{~cm}$ thick steel. There are no aperture restrictions that prevent the beam from hitting the wall of the decay pipe. Failure of the decay pipe wall could potentially occur if the accident persisted for several hours. The impact of such an accident is high since there is no simple means of repairing the decay pipe. Therefore the decay pipe is filled with helium gas at atmospheric pressure to reduce the stress on the window since December 2007. The decay volume is surrounded by 2.5-3.5 $\mathrm{m}$ of concrete shielding. At the end of the decay pipe there is a water-cooled absorber with an aluminium core encased in steel to stop any remaining primaries and undecayed 
secondaries. Any muons passing through the absorber are stopped by $240 \mathrm{~m}$ of dense Dolomite rock before they reach the Near Detector cavern. Ionization chambers are used to monitor the secondary and tertiary particle beams. One array is located immediately upstream of the absorber, and three others are located at the muon alcoves, one downstream of the absorber, one after $8 \mathrm{~m}$ of rock, and a third after an additional $12 \mathrm{~m}$ of rock. The first array monitors the remnant hadrons at the end of the decay pipe, and the other three arrays monitor the tertiary muons from $\pi$ and $K$ decays. The layout of the NuMI beam facility is shown in Fig.2.3.

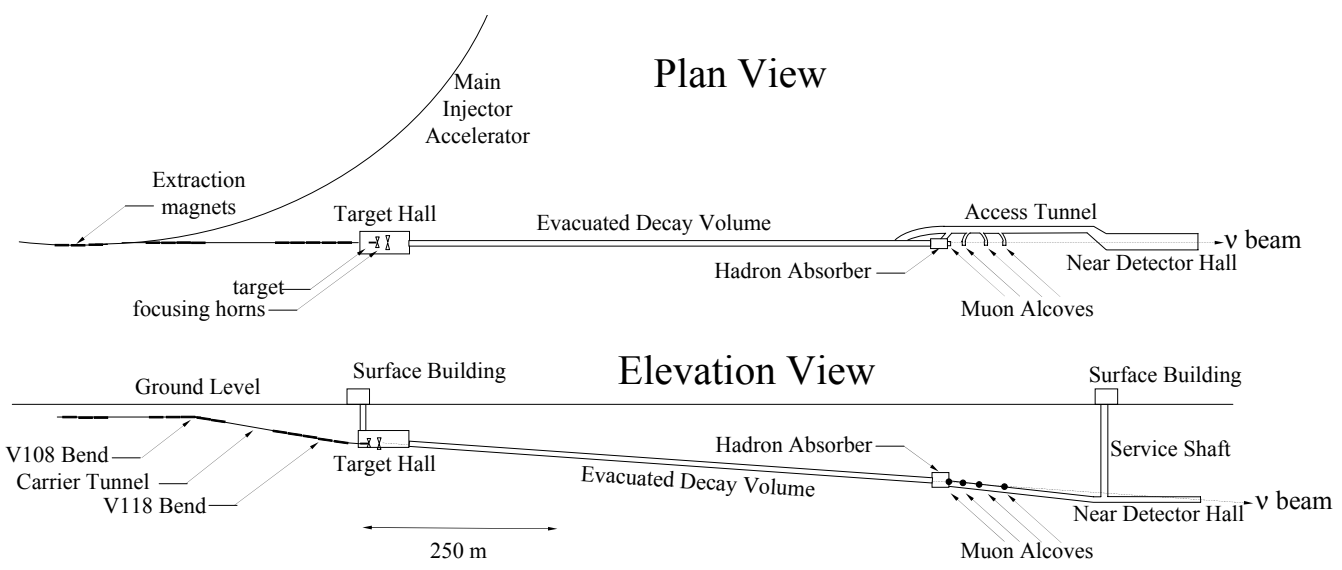

Figure 2.3: Plan and elevation views of the NuMI beam facility. A proton beam is directed onto a target, from which the secondary pions and kaons are focused into a decay volume via magnetic horns. Ionization chambers at the end of the beam line measure the uninteracted primary beam, secondary hadron beam and tertiary muon beam. Plot taken from [88].

The beam has been designed so as to adjust the energy spectrum of neutrinos in order to maximize sensitivity to oscillation parameter $\Delta m^{2}$. The relative placement of the two horns and the target optimizes the momentum focus for pions, hence the peak neutrino beam energy. Most of the time the beam line is configured in the "Low Energy" mode with $\left\langle E_{\nu}\right\rangle \sim 4 \mathrm{GeV}$. Moving the target upstream directs smaller-angle, higher-momentum particles into the magnetic fields of the focusing horns, resulting in a higher-energy neutrino beam, as shown in Fig.2.4. Different energy configurations 
are important for quantifying various systematic errors on the predicted neutrino energy spectrum.

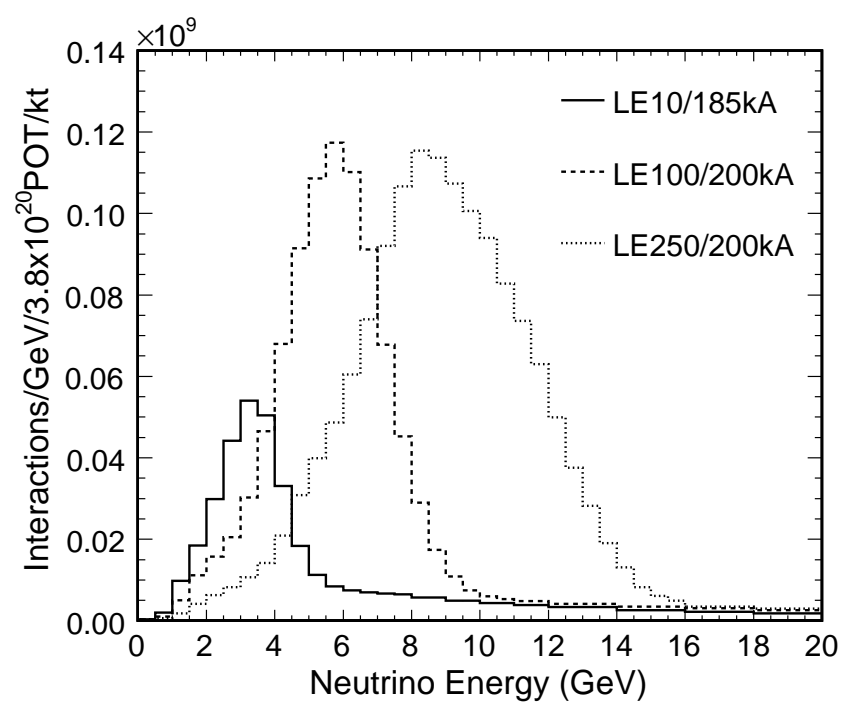

Figure 2.4: Calculated rate of $\nu_{\mu}$ charged-current interactions in the MINOS Near Detector. Three spectra are shown, corresponding to the low, medium, and high neutrino energy positions of the target. In these configurations, the target is located 10, 100, and $250 \mathrm{~cm}$ upstream of its fully-inserted position. Plot taken from [58].

The flavor composition of the beam will be predominantly $\nu_{\mu}$ (92.9\%). Small contributions of $\bar{\nu}_{\mu}(5.8 \%)$ will come from $\mu^{+}$decays and target-produced $\pi^{-}$decays. A $1.2 \% \nu_{e}$ component of the beam results from $\mu^{+}$decays and target-produced $K_{e 3}^{+}$ decays. The contribution from $\bar{\nu}_{e}$ is small $(0.1 \%)$.

\subsection{The MINOS Detectors}

The MINOS experiment uses two detectors to record the interactions of neutrinos in the NuMI beam. A third detector, called the calibration detector, was exposed to test beams at CERN to establish detector response to hadrons, electrons and muons with momenta in the range $0.2-10 \mathrm{GeV} / c$. The Near Detector at Fermilab is used to 
characterize the neutrino beam and its interactions and is located about $1 \mathrm{~km}$ from the primary proton beam target, the source of the neutrino parent particles. The Far Detector performs similar measurements $735 \mathrm{~km}$ downstream. The essence of the experiment is to compare the rates, energies and topologies of events at the Far Detector with those at the Near Detector, and from those comparisons determine the relevant oscillation parameters. The detectors have been designed to be as similar as possible to reduce systematic errors. In this section, we will first describe the main features of the MINOS detectors, and then move on to discuss the unique features of each individual detector.

\subsubsection{MINOS Detector Technologies}

All three MINOS detectors are steel-scintillator sampling calorimeters with an absorber layer of steel and an active layer of plastic scintillator. The steel and scintillator is arranged into a "sandwich" structure: a layer of $1 \mathrm{~cm}$ thick scintillator is attached to a layer of $2.54 \mathrm{~cm}$ thick steel to form a plane. There is a $2.41 \mathrm{~cm}$ air gap between two successive planes to relax the flatness tolerance of the steel plates. The scintillator is divided up into $4.1 \mathrm{~cm}$ wide strips, and each plane has one "view" of strips, with the next plane having the orthogonal view to give a three-dimensional tracking capability. The two views are at $45^{\circ}$ relative to vertical in order to avoid having strip readout connections at the bottom of the detector. One view is referred to as "U view" and the other view is referred to as "V view".

The detectors use a solid scintillator that is made by extruding polystyrene into long thin strips. The polystyrene is doped with the fluors PPO (1\%) and POPOP $(0.03 \%)$. Fiber readout of extruded scintillator was chosen as opposed to direct readout of cast scintillator in order to reduce costs. Each scintillator strip has a 2.3 mm-deep by $2.0 \mathrm{~mm}$-wide groove cut into its wider edge which runs along the length of the strip. A $1.2 \mathrm{~mm}$ diameter wavelength shifting (WLS) fiber-optic cable is glued 
into the groove. WLS fibers minimize self-absorption by absorbing light peaked at $420 \mathrm{~nm}$ and re-emitting it at a wavelength beyond $470 \mathrm{~nm}$. The blue photons from the scintillator are absorbed in the WLS fiber and re-emitted isotropically. Those resulting photons whose directions fall within the total internal reflection cones are transported along the fiber to the edges of the detector, subsequently being routed to the photodetectors. The scintillator surface is covered by a thin $(0.25 \mathrm{~mm})$ coextruded titanium-dioxide $\left(\mathrm{TiO}_{2}\right)$-loaded polystyrene layer that serves as a diffuse reflector. The scintillator and $\mathrm{TiO}_{2}$ coating are co-extruded in a single process, a standard technique in the plastics industry. The fiber must be completely contained inside the groove to ensure efficient light collection. A specularly reflective strip of aluminized Mylar tape is placed over the groove after the WLS fiber has been glued in place. The cutaway drawing of a single scintillator strip is shown in Fig.2.5.

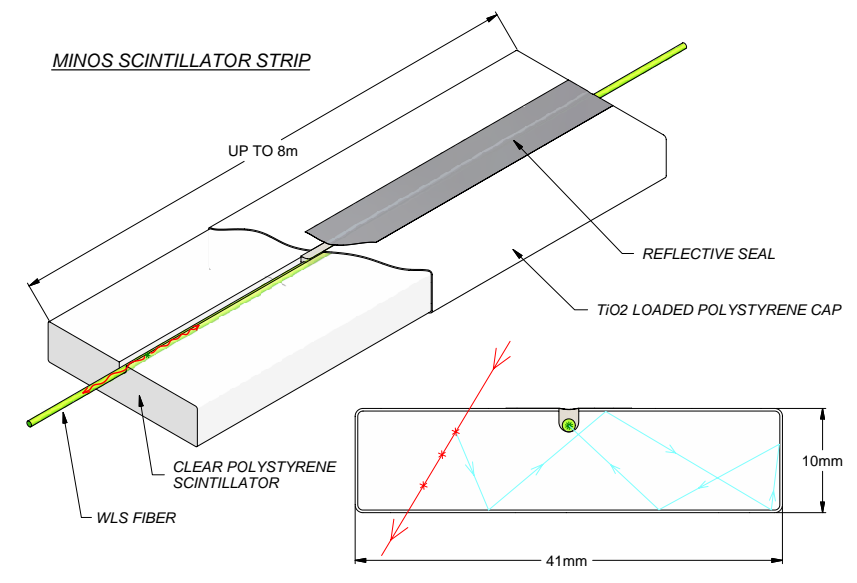

Figure 2.5: Cutaway drawing of a single scintillator strip. Light produced by ionizing particle is multiply reflected inside the strip by the outer reflective coating. Light absorbed by a WLS fiber is re-emitted isotropically. Those resulting photons whose directions fall within the total internal reflection cones are transported along the fiber to the edges of the detector, subsequently being routed to the photodetectors. Plot taken from [89].

The scintillator strips are encased in light-tight aluminium $(0.05 \mathrm{~cm}$ thick $)$ modules, which contain between 20 and 28 strips. After exiting the strips the WLS fibers 
run together in a manifold before they terminate in a connector. Clear fiber cables connect to the module and transmit light from the edges of the detector to centralized locations where the multi-anode photomultiplier tubes (PMTs) and readout electronics are mounted. A light injection system illuminates the WLS fibers near their ends with LED-generated UV light to monitor the stability and linearity of the PMTs. The MINOS detectors are read out by Hamamatsu 64-anode (M64) PMTs for the Near Detector and 16-anode (M16) PMTs for the Far Detector. The PMTs are housed in light-tight, steel enclosures containing clear fiber bundles which are interlaced from cable connectors to PMT pixels. In the Near Detector each M64 resides in an individual enclosure. In the Far Detector each enclosure (called a "MUX box") houses three M16 PMTs. Fig.2.6 illustrates the scintillator system readout for a module.

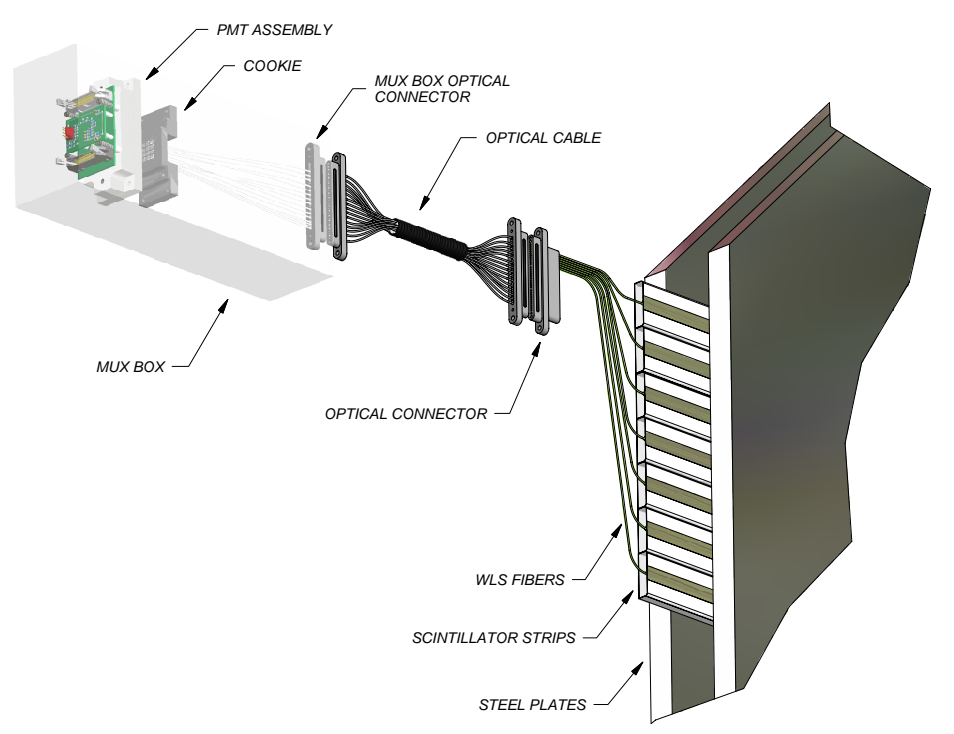

Figure 2.6: Schematic drawing of the scintillator system readout for a module. Plot taken from [89].

We now briefly discuss the electronics used in MINOS, then focus on the unique features of each detector in the next few sections. The phototube signals are digitized using a modified version of the Viking VA chips made by the Norwegian company 
IDEA ASA at the Far Detector [90] and FNAL "QIE" chips [91] at the Near Detector to meet the faster demands of the high event rate at the near site. In both cases, circuitry is installed to inject known amounts of charge into the digitization circuits for calibration of the digitization. Data are gathered into custom mid-level VME cards for readout by the data acquisition. The data acquisition reads charge and time information (called "digits") for each phototube signal, combines such data from all parts of the detector, and arranges them into time-ordered "snarls" of data that correspond to physical events.

Both Near Detector and Far Detector are magnetized with a current carrying coil producing an average magnetic field of 1.3(1.4) T in the Near(Far) Detector. A coil running down the center of the detector and back outside of the detector is used to create the field. At the Near Detector the coil is offset by $50 \mathrm{~cm}$ from the detector center so as to give a larger fiducial volume around the neutrino beam spot. MINOS is unique in that it is the only large, underground detector with a magnetic field. The magnetic field allows charge separation between the $\mu^{+}$and $\mu^{-}$, and therefore charged current interactions of $\nu_{\mu}$ and $\bar{\nu}_{\mu}$. A comparison of atmospheric oscillation properties for the two would be a test of CPT symmetry in neutrino masses and mixing. The magnetic field also allows the muon momentum to be measured through the curvature of tracks that are only partially contained within the detector. It also focuses negative particles (i.e. $\mu^{-}$) created in the beam $\nu_{\mu} \mathrm{CC}$ interactions increasing the proportion that are fully contained. The energy resolution on the curvature measurements is approximately $14 \%$ at $10 \mathrm{GeV}$ muon momentum. Multiple scattering of the muons in the steel is the predominant limitation on the accuracy of these measurements. For muons that stop in the detector a much better measurement of their momenta can be obtained from a range measurement. The energy resolution for stopping muons from a range measurement is approximately $6 \%$. In the energy range of interest, the average muon momentum is roughly $2 \mathrm{GeV} / \mathrm{c}$. The low energy muons are most 
likely to stop in the detector and their momenta are measured through the range accordingly.

The data acquisition (DAQ) and timing system synchronize and continuously read out the front-end electronics. Software triggering in the DAQ provides flexible event selection and data processing. Various triggers are implemented in the DAQ system. The triggers fall into three categories: special triggers for debugging and calibration, bias-free triggers based on spill signals or spill times to gather beam events, and triggers based on the clustering of hits in the detector to gather out-of-spill events. GPS timestamps allow data from the two detectors to be synchronized with the beam pulses. The two detectors have different front-end electronics due to the disparate rates of neutrino interactions and cosmic-ray crossings at the two sites.

\subsubsection{The Far Detector}

The Far Detector has a mass of 5.4 kt. It is the largest of the three MINOS detectors and is located $714 \mathrm{~m}$ below the Earth's surface (2070 m water equivalent) in the Soudan Underground Laboratory, Northern Minnesota. It consists of $4868 \mathrm{~m}$ wide octagonal planes, arranged in 2 super-modules of 249 and 237 planes, of which 248 and 236 are instrumented with scintillator. The division of the detector into two super-modules is made due to restrictions on the length of the magnetic coil, thus each super-module is independently magnetized. The first and second super-modules were completed in August 2002, and June 2003, respectively. Collection of cosmic-ray and atmospheric neutrino data for each super-module has begun since shortly after the completion of each super-module. Each of the scintillator planes is divided up into 192 strips. The 192 scintillator strips are encased in 8 modules. The center four modules contain twenty $8 \mathrm{~m}$ long strips. The outer modules contain 28 strips varying in length from $3.4 \mathrm{~m}$ to $8 \mathrm{~m}$. The center two modules must provide clearance for the detector's magnet coils. A semi-circular hole of radius $197 \mathrm{~mm}$ is cut into 
the aluminum covers of the affected modules and short lengths of scintillator strips passing through the hole are also cut away. However, the WLS fibers passing through the affected strips are not cut. Rather, a "bypass" channel routes them around the hole.

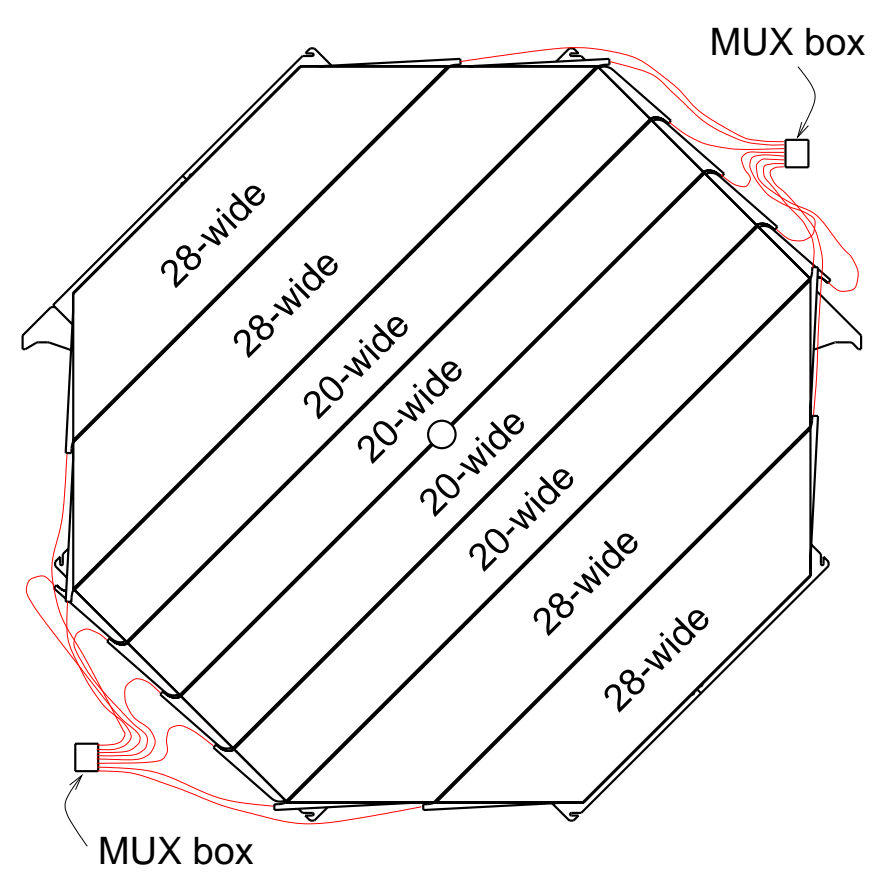

Figure 2.7: Layout of far detector scintillator modules. The center four modules contain twenty strips and the outer modules contain 28 strips.

The scintillator strips are read out at both ends by Hamamatsu M16 PMTs with 16 pixels. To reduce the large instrumentation load, a multiplexing technique (optical summing) is employed so that 8 strips from each plane are read out by the same PMT pixel. This is feasible because the transverse spread of hadronic/electromagnetic showers from beam neutrinos is limited to a $\sim 1 \mathrm{~m}$ region. To enable determination of which strip was actually hit, the 8 strips read out by a single pixel on one side of the detector are read out by 8 different pixels on the other side.

The front-end electronics (FEE) at the Far Detector [90] were specifically designed 
for the low rate underground environment. A block diagram of the readout structure is shown in Fig.2.8. The readout is based on the front-end ASIC VA32_HDR11 (short

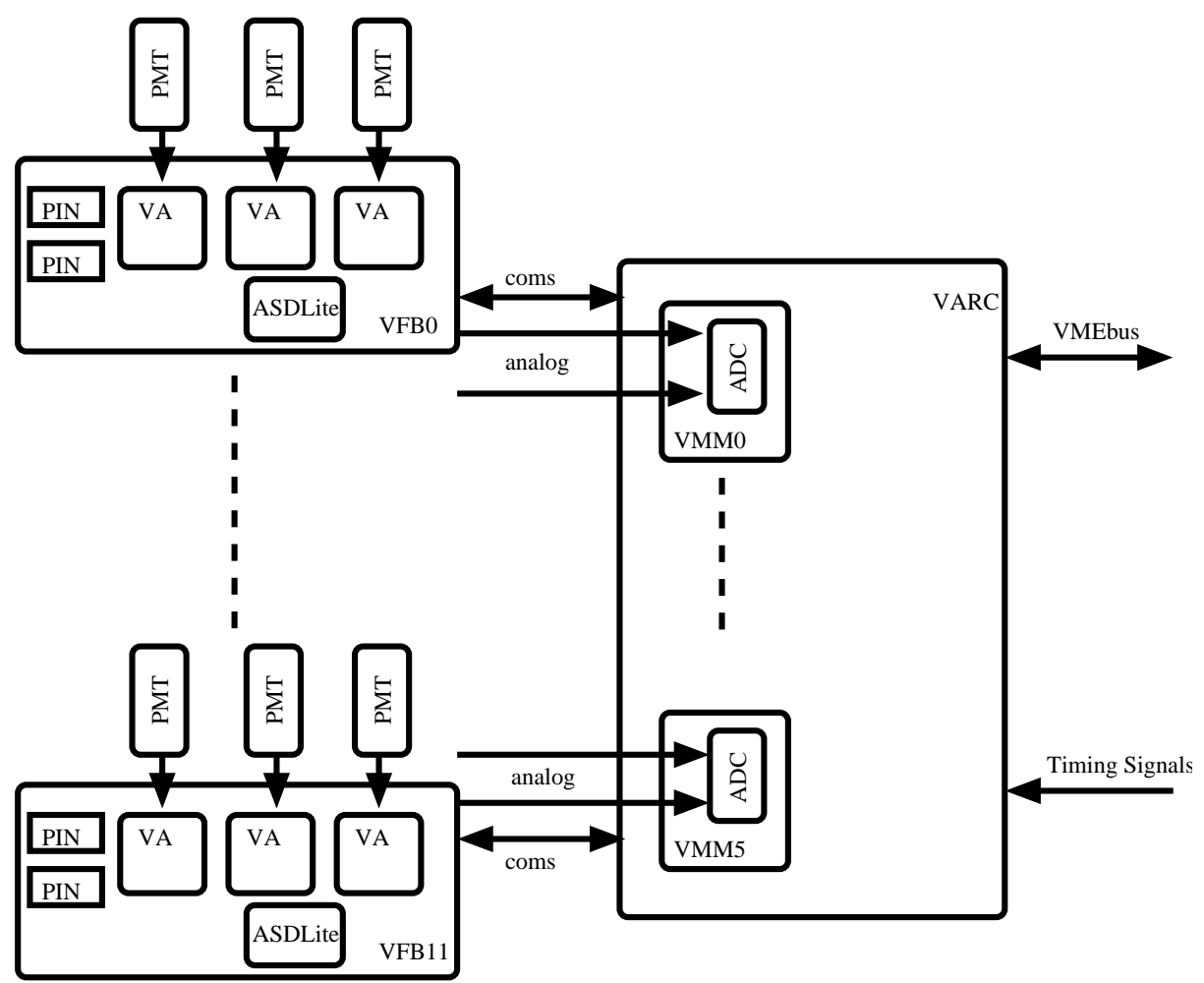

Figure 2.8: Schematic overview of the MINOS far detector readout electronics. Three PMTs are connected with short flat ribbon cables to the VFB, which also houses two PIN diodes to monitor the light level of the light injection system. The VA ASIC amplifies and holds the PMT signals, which are multiplexed via an analog link onto an ADC on the VARC. The VFB is controlled through a digital link by the VARC. Plot taken from [89].

VA chip), developed in collaboration with the Norwegian company IDEA ASA. Each VA chip is responsible for sampling and holding the signals from one of the three PMTs in a MUX box. Three VA chips are mounted onto a single VA Front-end Board (VFB), located on the outside of the PMT MUX box. The VFB provides support circuitry for power distribution and biasing of the VA chips. It also contains a discriminator chip ASDLite ASIC, which compares the dynode signals from the 
PMTs with a common programmable threshold to provide a discriminated signal for time-stamping and readout initiation. The VFB is operated in slave mode and fully controlled by the VA readout controller (VARC) described below.

The analog signals from the VA chip are multiplexed onto an ADC, which is located on a VA Mezzanine Module (VMM). Two VFBs are connected to each VMM. The VARC houses 6 VMMs and controls the signal digitization, triggering, timestamping and bias of the VA chips. Each VARC can thus service up to 36 PMTs of 16 channels each.

The VARC is implemented as a $9 \mathrm{U}$ VME card. Three VARCs, a timing card, and a Motorola VME processor share a single VME crate. The VARC receives the discriminated dynode signal of each PMT. It time-stamps these signals with an effective $640 \mathrm{MHz}$ TDC, and then generates the hold signal for the VA ASIC. The signals held in the VA ASIC are then multiplexed to a commercial 14-bit $10 \mathrm{MHz}$ ADC. The digitization sequence is started if the VARC receives at least two discriminated dynode signals from different PMTs in a 400 ns window. This so-called 2-out-of-36 trigger reduces the dead time due to dark noise in the PMTs and fiber noise in the scintillator, without compromising the recording of physics events. The entire detector readout is synchronized by a $40 \mathrm{MHz}$ optical timing distribution signal slaved to a GPS clock from TrueTime [93].

Once the data are digitized they are transmitted to a local FIFO and stored there for further processing. The pedestal is subtracted and data above an individually settable sparsification threshold are written to an on-board VME memory. This memory is read out by the DAQ system. The VARC also controls pedestal and charge injection calibration runs.

To aid the atmospheric neutrino analysis in the Far Detector, a cosmic ray veto shield has been erected around the top and sides of the detector. This allows cosmic rays to be tagged and reduces the cosmic ray background by a factor of approximately 
100. The shield is made of the same scintillator strips as the main detector and the data is read out in the same fashion. Due to the steepness of cosmic tracks and knowledge of the spill time, cosmic ray muons are not a significant source of background for the beam analysis, so shield information is not used for accelerator neutrino analysis.

\subsubsection{The Near Detector}

The Near Detector has a mass of $0.98 \mathrm{kt}$. It is located a short distance from the neutrino source at Fermilab, $100 \mathrm{~m}$ below the surface (225 m water equivalent). The design of the near detector takes advantage of the high neutrino flux at this location to define a relatively small target fiducial volume for selection of events for the near/far comparison. At the beam intensity of $2.2 \times 10^{13} \mathrm{POT} /$ spill, an average of 16 neutrino interactions occurred in the Near Detector during each $10 \mu \mathrm{s}$ spill in the low energy beam configuration [58]. The Near Detector consists of 282 planes. It has an elongated octagonal cross-section, $3.8 \mathrm{~m}$ high and $4.8 \mathrm{~m}$ wide. Only 153 of the 282 planes are active. The upstream 120 planes of the detector, the calorimeter section, contains the target fiducial volume in which every plane is instrumented. The downstream part, the spectrometer section, is used to measure the momenta of energetic muons and has only every fifth plane instrumented with scintillator. Active planes are instrumented with four distinct scintillation module patterns: full U-view (FU), full V-view (FV), partial U-view (PU), and partial V-view (PV). The area of partial coverage is set to ensure the complete measurement of neutrino events occurring in the near detector fiducial volume. The full-view coverage extends around the coil hole in order to track long range muons downstream of neutrino interactions. The layouts of different scintillator modules are shown in Fig.2.9 

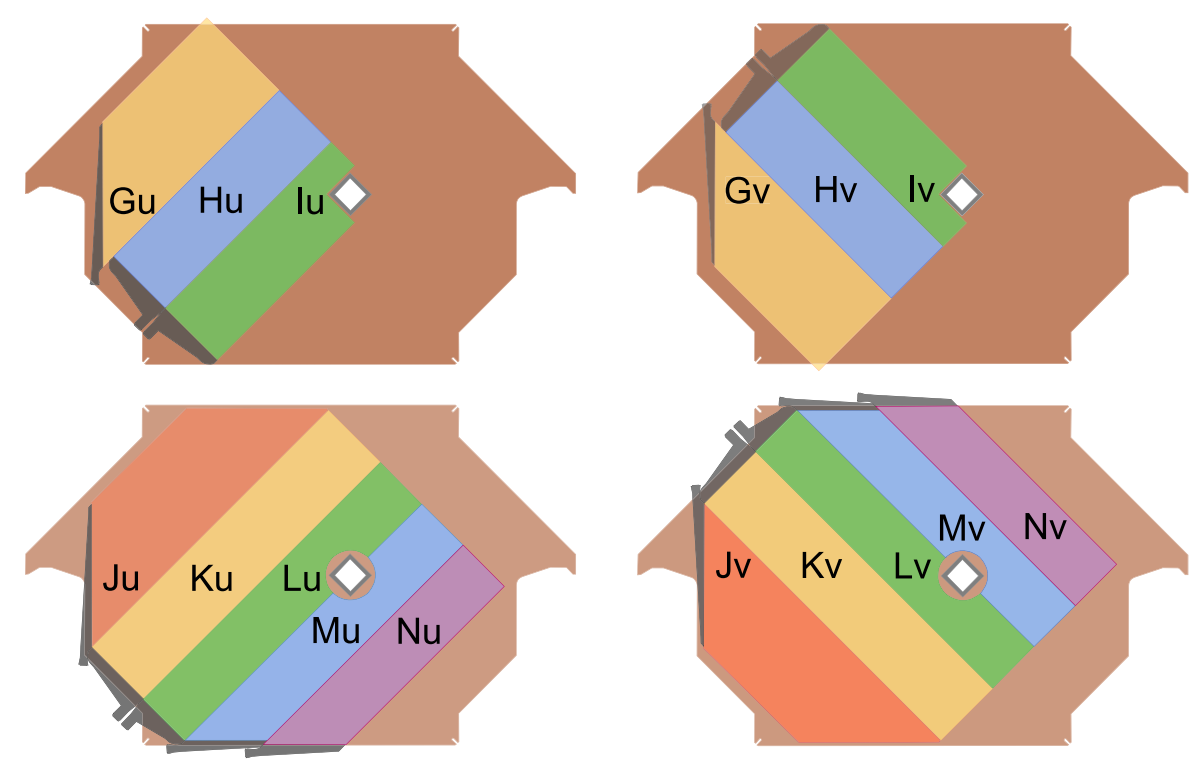

Figure 2.9: The four different configurations of planes used in the Near Detector, showing the different layouts of the scintillator modules. The upper two figures show partially instrumented planes ("calorimeter region") while the lower two figures show the fully instrumented ones ("tracking region"). The G-N notations denote the different shapes of the scintillator modules. The beam is centered midway between the coil hole and the left side of the plane, hence the scintillator need only cover that area in the target region. Plot taken from [89]. 
For data analysis, the calorimeter section is divided into three longitudinal sections: planes 1-20 are the "veto" section, used to exclude events that originate upstream of the detector; planes 21-60 represent the "target" region, as all neutrinoinduced showers which occur here are contained within the length of the detector; planes 61-120 complete the calorimeter section and are used to contain and measure the hadronic showers of neutrino events in the target region. Every plane in the calorimeter section is instrumented to enable accurate tracking and calorimetry. The spectrometer section of the Near Detector, planes 121-281, is used to measure the momenta of energetic muons and only every fifth plane is fully instrumented with scintillator. The four logical sections of the Near Detector are shown in Fig.2.10.

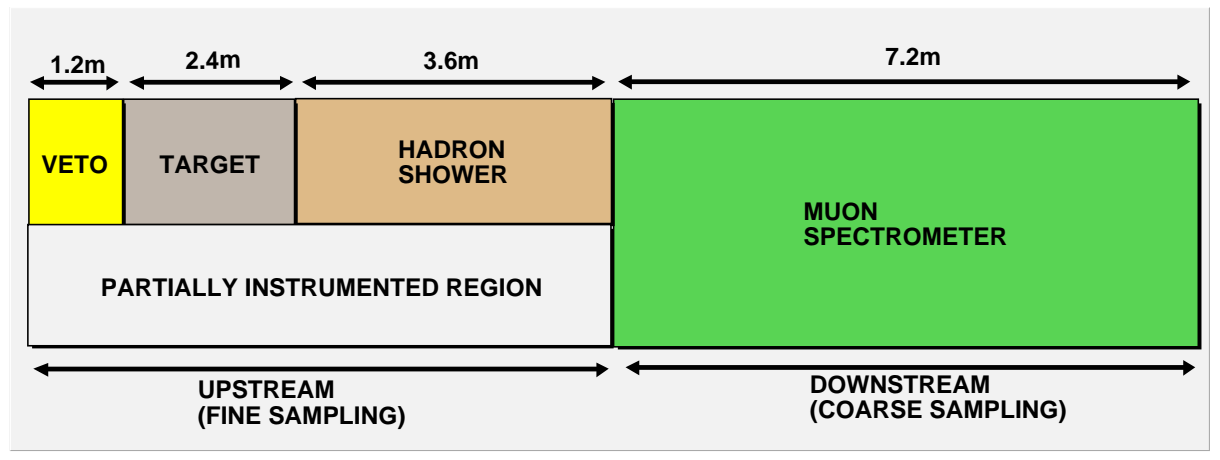

Figure 2.10: Four logical sections of the Near Detector. The partially instrumented region only has scintillator in every 5th plane. (Plot courtesy Debdatta Bhattacharya)

In contrast to the Far Detector, scintillator strips in the Near Detector need only be read out on one end; their relatively short lengths (typically $2.8 \mathrm{~m}$ compared to $8 \mathrm{~m}$ at the Far Detector) ensure that enough photons reach the PMTs to detect the passage of a minimum ionizing particle efficiently. The other end of the strip is covered with reflective aluminized mylar tape to increase the light yield. The scintillator strips are read out by Hamamatsu M64 PMTs with 64 pixels. A partially-instrumented plane has 64 strips so is read out by a single PMT. A fully-instrumented plane has 96 strips so is read out by 1.5 PMTs. Furthermore, to reduce the instrumentation, 
a 4-fold electrical summing technique is employed in the muon-spectrometer region. Each PMT is split into groups of 4 pixels and the signals from the 4 anodes are fed into a single electronics channel. The consequence of the electrical summing is that a "seed" track in the forward region is needed to project into the muon-spectrometer for unambiguous reconstruction.

Due to the multiple events occurring in each beam spill, dead-timeless, high-speed front-end electronics is required for the Near Detector. In the Near Detector front-end electronics [91], each PMT pixel is digitized continuously at the frequency of the beam RF structure of 53.103 MHZ (18.83ns). This is achieved with an individual front-end channel unit consisting of a small mezzanine printed circuit board (PCB) called a

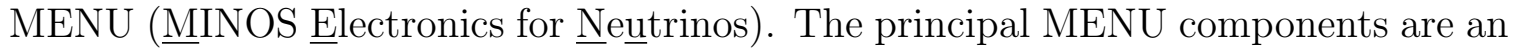
ASIC named the Charge Integrator and Encoder (abbreviated "QIE"), a commercial flash analog-to-digital converter ("FADC"), and a data buffer. A MENU board also contains circuitry for measuring source current and circuitry for injecting DC current into the QIE for performing electronics calibration. Sixteen MENUs reside on a VME

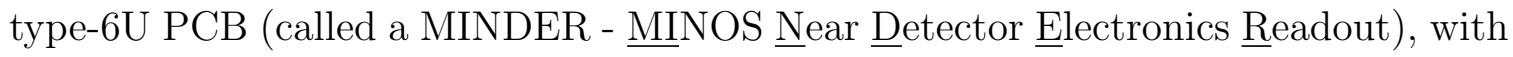
four MINDERs required for each fully used M64 PMT.

The QIE input signal current, $I$, is split into eight binary-weighted "ranges" with values $I / 2, I / 4, I / 8, \ldots$, and integrated onto a capacitor for each range. A bias current is added to ensure that the capacitor voltage on one and only one range is within the predetermined input limits of the FADC. The QIE selects that voltage for output to the FADC, and also outputs a 3-bit number representing the range value. Each QIE is equipped with four independent copies of the current splitter, integration, and output circuits to permit continuous dead-timeless operation. Each QIE data word consists of 13 bits including an 8-bit FADC value, a 3-bit range value, and a 2-bit code for the identification of the current splitter and capacitor circuit (know as "CapID"). 
Data from the front-end, consisting of range, FADC value, and CapID, are transfered to VME type-9U modules called MASTERs (ㅆNOS Acquisition, $\underline{\text { Sparsifier, }}$ and Time-stamper for Event Readout), which read out up to eight MINDERs each. The MASTERs linearize the data words using a lookup table which represents the results of a charge injection calibration of each MENU. The resulting linearity is better than $0.5 \%$ over the entire dynamic range.

To provide uniformity, a centralized Near Detector clock system is used to distribute a continuous $53 \mathrm{MHz}$ reference, spill signals, and other control signals to all front-end modules. Clock signals are also used to synchronize the readout of data by the VME processors and the DAQ system. The Near Detector clock is synchronized to the Fermilab accelerator but the phase of timing signals relative to an independent GPS system is used to allow accurate reconstruction of the absolute UTC event time.

\subsection{Detector Calibration}

The detector calibration is very important for the MINOS measurements. The MINOS detectors measure hadronic and electromagnetic shower energy by calorimetry. The relative detector energy calibration is critical for neutrino oscillation studies that rely on comparisons of energy spectra and event characteristics in the near and far detectors. The task of calibration for MINOS comprises: removing detector variations within each detector, relating energy deposits from different detectors (relative calibration) and finally translating detector responses for different types of particles to energy in $\mathrm{GeV}$ (absolute calibration).

\subsubsection{Relative Calibration}

Fig.2.11 shows the relative calibration scheme for MINOS data. The main branch of this calibration is to convert raw ADC's into calibrated Muon Energy Units (MEU). 


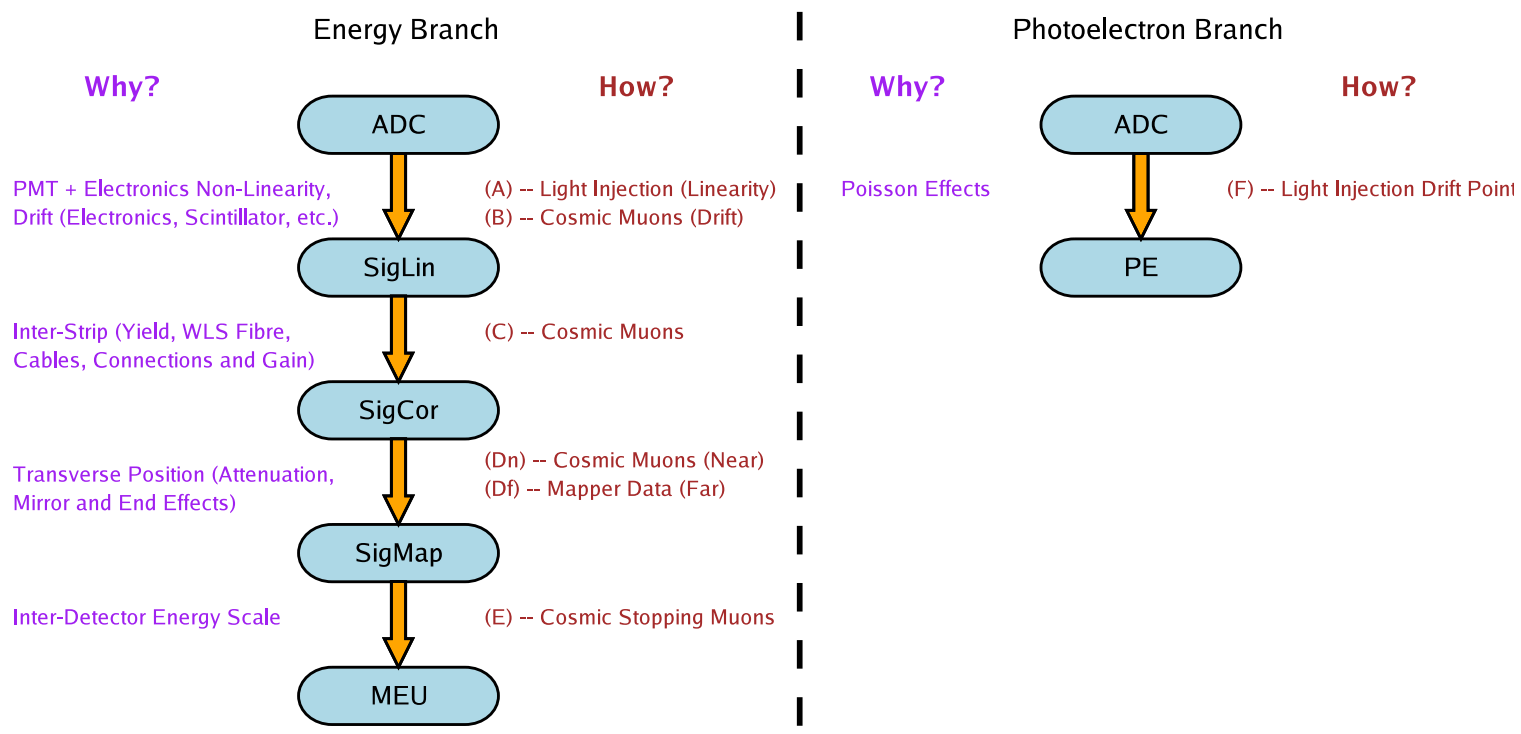

Figure 2.11: Calibration scheme for data. The main branch is the energy branch which converts raw ADC's into calibrated Muon Energy Units (MEU). The secondary branch converts ADC to photoelectrons. Plot taken from [92].

The first step towards achieving this is to make the response of each individual detector uniform. The calibration corrects for scintillator light output variations as well as nonuniformities of light transmission and collection in the fibers, PMTs and readout electronics. The charge-injection system is used to measure the linearity of the electronics. The light-injection system is used to measure the linearity and time variation of the readout response. The cosmic ray muons are used to measure scintillator strip light output variations with time and position, specifically to record interstrip and intrastrip nonuniformities. The next step is to relate energy deposits in one detector with those in another. This can be done by using particles of known energy and comparing the detector responses. MINOS uses stopping muons from cosmic rays to do this inter-detector calibration. 


\section{The Light-injection Calibration System}

A light-injection (LI) calibration system has been designed for the MINOS detectors to map the linearity of the instrumentation, to monitor the stability of the PMTs and electronics over time, to evaluate the single-photoelectron gain, and to monitor the integrity of the optical path and readout system. [94]. The system is based upon pulsed blue light-emitting diodes (LEDs). A rack-mounted box, known as a "pulser box", contains a set of 20 LEDs, each of which has an optical fanout allowing it to illuminate multiple individual fibers. These fibers carry light to a set of optical connectors on the back panel of the pulser box.

From the pulser box, optical fibers carry the light to the outer edges of the MINOS detector. A set of highly reflective cavities situated there, "the "light-injection modules" (LIM), allows the blue LED light to illuminate the green wavelength-shifting fibers, thus producing pulses of light that mimic the signals from the scintillator.

There are two different LI data taking modes. In order to monitor the changes in gains of PMTs and electronics as well as for other transient instabilities, the LI system periodically pulses the fiber at every strip end. Each Far Detector strip end is pulsed 1000 times at $200 \mathrm{~Hz}$ every hour and each Near Detector strip end is pulsed 2000 times at $100 \mathrm{~Hz}$ every two hours. The pulse intensity of each LED is tuned such that a PMT pixel receives approximately 50 photoelectrons per pulse on average. The operational mode is referred to as "Drift Point". The LI system is also used to map the non-linearity of PMT response. Each strip end fiber is pulsed 1000 times at many different light levels once a month at both near and far detectors. The pulse intensity settings for each LED are tuned so that the average response of the strips connected to it covers the full dynamic range of interest. This operational mode is referred to as "Gain Curve".

The intensity of injected light is monitored by PIN photodiodes that are read out simultaneously with the PMTs. Although the PIN photodiodes themselves are 
measured to be quite linear, non-linearities on the order of 1-2\% are apparent in the readout response from both detectors because of the electronics noise. In the Near Detector, PIN diodes are amplified with high gain in order to suppress the electronics noise. In the Far Detector, the readout is still very non-linear even with high-gain PIN diodes. Therefore, the PIN diodes are not used in the Far Detector. Instead, the readout of the other end (far end) of the flashed strip end (near end) is used to monitor the intensity of injected light [95].

I have been working on the Near Detector LI system together with the former Stanford postdoc Simona Murgia since 2004. My main contribution was the tuning of pulse intensity settings for LEDs. I also helped with the improvements of the electronics for the PIN diodes readout in the Near Detector.

\section{Cosmic Ray Muon Calibrations}

Cosmic ray muons are used at each detector to measure scintillator related quantities. Through-going cosmic ray muons have an average energy of $200 \mathrm{GeV}$ at the Far Detector and a rate of $0.5 \mathrm{~Hz}$. At the Near Detector, the mean energy is $55 \mathrm{GeV}$ and the rate is $10 \mathrm{~Hz}$.

Cosmic ray muons are used to track the response of each detector on a daily basis. This "drift" calibration is performed by measuring the total pulse height per plane of through-going cosmic ray muons. The daily median of the pulse height per plane is computed, and the relative change in this quantity is used to compute the drift. This measured drift encompasses changes due to the scintillator, WLS fiber, PMTs and electronics.

Through-going cosmic ray muon data are used to measure the strip-to-strip (channelby-channel) time dependent response of the detector. This calibration relates the mean response of each strip to the detector average. This calibration incorporates several detector effects that vary channel-by-channel, including scintillator light yield, 
WLS fiber collection efficiency, readout fiber attenuation, PMT quantum efficiency and PMT gain. Cosmic ray muon tracks are used to measure the mean light level at each strip end. To remove known spatial and angular dependencies, attenuation and path-length corrections are applied to each hit such that the calibration constant is calculated to be the mean response of a muon of normal incidence traveling through the center of the strip. Once the strip-to-strip calibration is performed, the response from the center of any strip of a particular length is the same.

Throughgoing cosmic ray muon data are also used to correct the variation in light caused by attenuation along the WLS fiber in a scintillator strip. The pulse height from a strip hit by a track is plotted as a function of the longitudinal track position. These data are fit to a double exponential:

$$
A(x)=A_{1} e^{-x / L_{1}}+A_{2} e^{-x / L_{2}} .
$$

where $x$ is the track position along the strip and $L_{1}, L_{2}$ stand for two attenuation lengths. A fit is performed for each strip and the resulting parameters are used to correct the data. One example for the Near Detector is shown in Fig.2.12.

The final stage in the relative calibration chain, before a conversion to absolute energy deposition in $\mathrm{GeV}$, is the inter-detector calibration. This calibration step serves to relate energy deposits in one detector with those in another. Stopping cosmic ray muon data are used for this task because they are abundant enough at all detectors and their energy depositions in each plane can be accurately determined from range measurements. A "track window" technique was developed for this calibration. This technique measures the response of muons only when their momenta are between 0.5 and $1.1 \mathrm{GeV}$. This avoids using data from the end of the track where the rapid increase in ionization occurs. After the inter-detector calibration, the three detectors will give an identical response for the same particle of given energy (to within the 


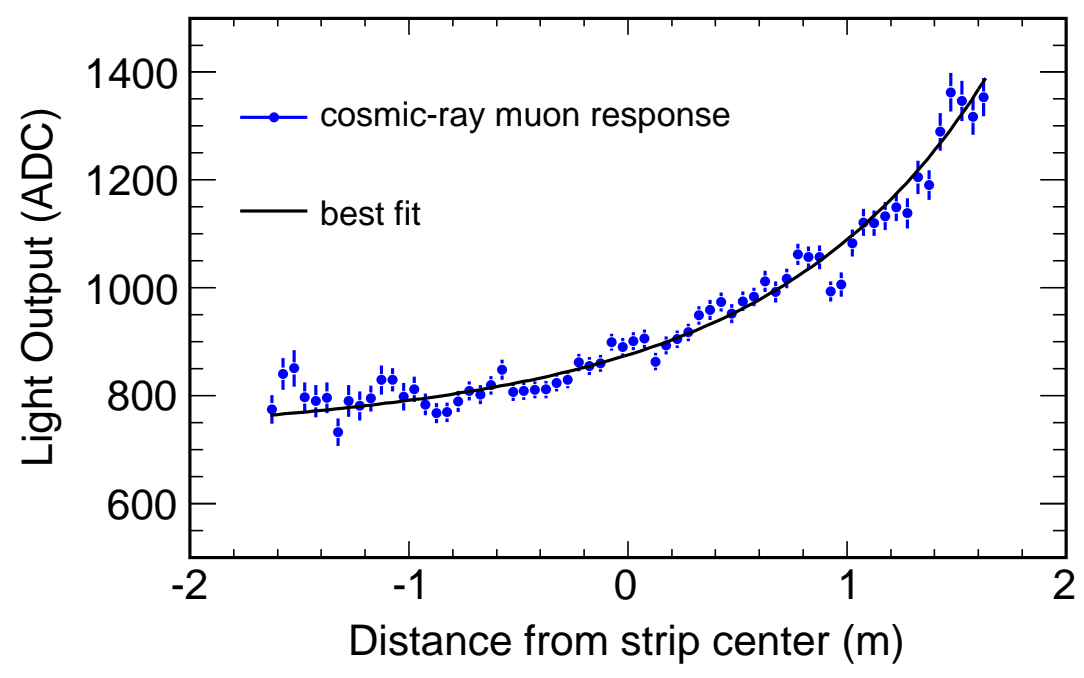

Figure 2.12: Detector response to cosmic ray muons as a function of distance from strip center for a typical strip in the Near Detector. (Plot courtesy Jiajie Ling)

errors of the calibration). A universal energy unit, the Muon Energy Unit (MEU, also referred to as a Minimum Ionizing Particle, MIP) is defined as the detector response to a perpendicular $1 \mathrm{GeV}$ muon traversing 1 plane of scintillator.

\subsubsection{Absolute Calibration}

MINOS physics analyses require a good understanding of the detectors' response to muons, electrons and hadrons with energies below $10 \mathrm{GeV}$. One needs to understand not only the overall energy scale and resolution, but also the event shape characteristics. The calibration detector [96] was exposed to test beams at CERN to establish the response to hadrons, electrons and muons with momenta in the range 0.2-10 $\mathrm{GeV} / c$. The measurements were used to normalize Monte Carlo simulations and to establish the uncertainty in the hadronic and electromagnetic energy scales. The data from the calibration detector were compared with events simulated using the same GEANT3 [100] based Monte Carlo used for the near and far detectors. 


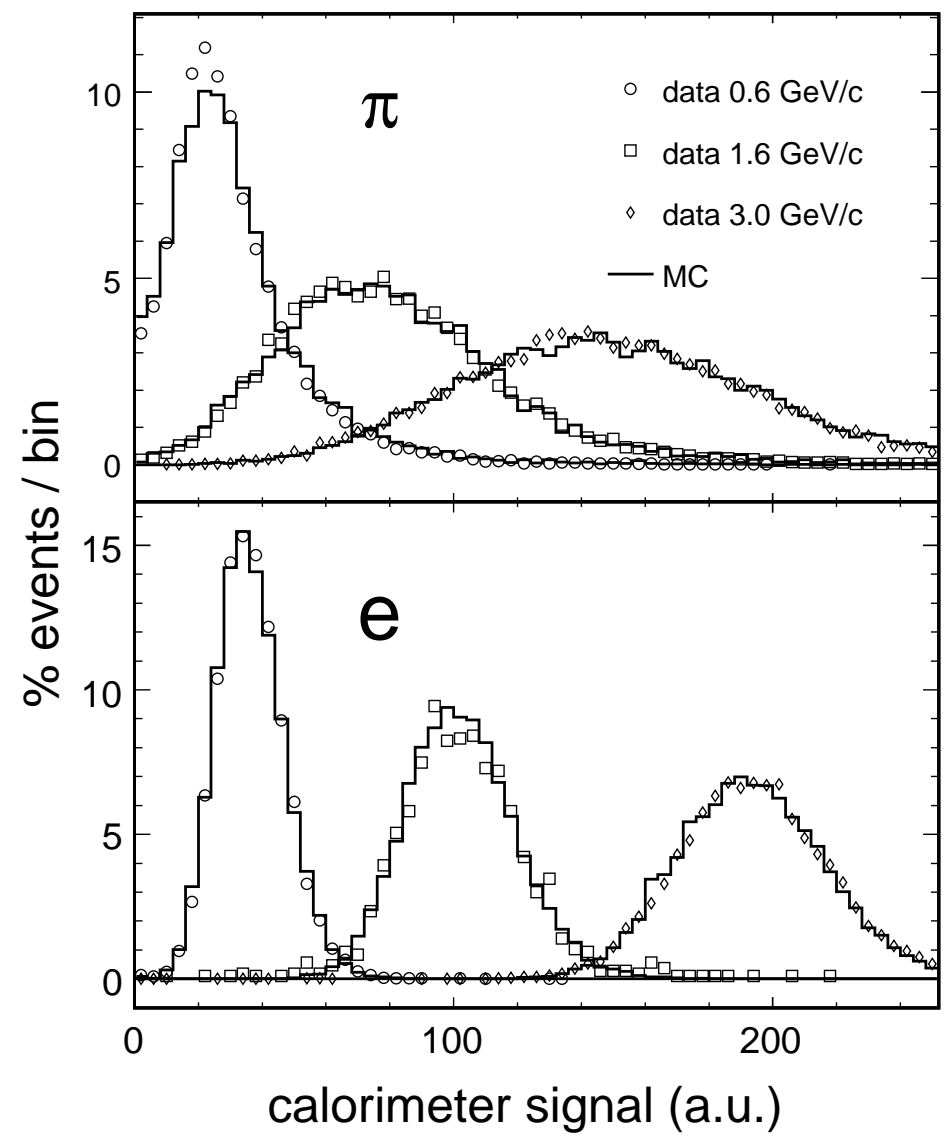

Figure 2.13: MINOS calorimetric response to pions and electrons at three momenta. The calorimeter-signal scale is in arbitrary units. The data (open symbols), obtained from the calibration detector exposure to CERN test beams, are compared to distributions from Monte Carlo simulations. Pion induced showers are simulated using the GCALOR shower code. Plot taken from [89]. 
Fig.2.13 shows the measured detector response to pions and electrons compared with the simulation result. The simulated calorimetric response to electrons agreed with the data to better than $2 \%$ in the electron momentum range $0.2-10 \mathrm{GeV} / \mathrm{c}$ [97]. Pion and proton induced showers were compared with events simulated using the GHEISHA, GEANT-FLUKA and GCALOR shower codes. The GCALOR-based simulation was in best agreement with the data and was adopted as the default shower code. The Monte Carlo reproduces the response to pion and proton induced showers to better than $6 \%$ at all momentum settings [98]. The energy resolution was adequately reproduced by the simulation and may be parametrized as $56 \% / \sqrt{E} \oplus 2 \%$

for hadron showers and $21.4 \% / \sqrt{E} \oplus 4 \% / E$ for electrons, where $E$ is expressed in $\mathrm{GeV}$.

\subsection{Monte Carlo Simulation}

Monte Carlo (MC) simulation is an important part of all the MINOS analyses. We measure the neutrino interactions in the Near Detector and we use the MC to extrapolate the Near Detector spectrum to predict the unoscillated Far Detector spectrum to compare with data for evidence of spectral distortion. The prediction calculated must take into account the ND and FD spectral differences that would be present, even in the absence of oscillations, due to pion decay kinematics and beam line geometry.

The modeling of the neutrino beam-line includes a simulation of the hadrons produced by $120 \mathrm{GeV} / c$ protons incident on the NuMI target and the propagation of those hadrons and their progeny through the magnetic focusing elements, along the decay pipe and into the primary beam absorber allowing for decay of unstable particles. The production of secondary mesons in the NuMI target was calculated using the FLUKA05 [99] Monte Carlo, which has uncertainties at the 20\%-30\% level stemming from a lack of relevant thick target hadron production data. Particles 
exiting the target are recorded and later propagated in a GEANT3 [100] simulation of the NuMI beam-line (GNuMI). The simulation takes into account the effects of the magnetic focusing horns, surrounding shielding, decay pipe and beam absorber. The GEANT-FLUKA code is used to model the secondary interactions in the horn and the decay pipe as well as the full particle decay chains. Decays in which a neutrino is produced are saved and later used as input for neutrino event simulation in the Near and Far Detectors.

Neutrino interactions are modeled by the NEUGEN-v3 [101] program. NEUGEN simulates both (quasi-)elastic and inelastic neutrino scattering. The latter includes a Rein-Sehgal [102] based treatment of neutrino induced resonance production, charged- and neutral-current coherent pion production and a modified leading order deep-inelastic scattering (DIS) model [103] extended to improve the treatment in the transition region between DIS and resonant production. The neutrino-induced hadronic multiparticle production is simulated using the AGKY program [104], which employs the PYTHIA/JETSET model [105] to simulate interactions with high invariant masses and a phenomenological model to simulate interactions with low invariant masses. I worked on the AGKY model with other people in 2006 and I will describe this model in more detail in a later chapter since it is very important for the MINOS $\nu_{e}$ search. Hadrons produced in the neutrino scattering are allowed to interact while exiting the target nucleus ("final state interactions"). The final state interactions are calculated using the INTRANUKE code from within NEUGEN3-v3. The calculation incorporates pion elastic and inelastic scattering, single charge exchange and absorption [106].

The response of the detector is simulated using GEANT3 with the GCALOR [107] model of hadronic interactions. The simulation randomly samples neutrinos from the flux predicted by the beam simulation and traces them through the Near and Far Detector halls. The simulation includes a detailed geometric model of the 
detector. The position of individual scintillator strips was determined with a precision of approximately $1 \mathrm{~mm}$ using cosmic-ray tracks. The magnetic field is modeled via finite element analysis driven by bench measurements of the steel B-H curve.

The final step in the simulation chain involves photon generation in the scintillator, capture and transmission of photons through internal reflection in the WLS fibers and conversion of photons to photoelectrons in the PMTs. This step uses a program written in Object Orientated $(\mathrm{OO}) \mathrm{C}++$ and based in the ROOT framework. It simulates many features including PMT cross-talk (some signal can be detected on adjacent pixels to the one being illuminated), noise, non-linearity (PMT, VA and QIE) and triggers. The cross-talk modeling and tuning will be discussed in more detail in a later chapter because it has sizable impact on the background estimation in the $\nu_{e}$ appearance analysis.

\subsection{MINOS Physics Analyses}

The combination of the NuMI beam and the MINOS detectors, whose characteristics are summarized in the previous sections, provide an optimal tool to search for and to study neutrino oscillations. The design of the MINOS detectors was also guided by the interest in the nonaccelerator physics.

The main goals of the MINOS experiment are to verify the existence of neutrino oscillations in the region of parameter space suggested by the atmospheric neutrino experiments and to make a precision measurement of the parameters. The three main accelerator neutrino oscillation analyses in MINOS are:

- $\nu_{\mu}$ and $\bar{\nu}_{\mu}$ disappearance measurement (CC analysis)

- Search for sterile neutrinos (NC analysis)

- Search for $\nu_{e}$ appearance ( $\nu_{e}$ analysis) 
In this section, we will discuss the accelerator neutrino oscillation measurements that make use of the NuMI neutrino beam.

\subsection{1 $\nu_{\mu}$ disappearance measurement}

The primary goal of MINOS is to observe the oscillation-induced spectral distortion of the charged current $\nu_{\mu}$ interactions at the Far Detector [57, 58, 59]. From the comparison of the reconstructed neutrino energy spectra at the near and far locations the oscillation parameters $\left|\Delta m_{32}\right|^{2}$ and $\sin ^{2}\left(2 \theta_{23}\right)$ are extracted.

The measurement of the energy spectrum at the ND is used to predict the unoscillated spectrum at the FD. The latest results use data recorded between May 2005, and July 2007 [59]. Over this period, a total of $3.36 \times 10^{20}$ protons on target (POT) were accumulated for this analysis. At this exposure, a total of 848 events were observed in the FD for all energies $0-120 \mathrm{GeV}$ produced by the NuMI beam, compared to the unoscillated expectation of $1065 \pm 60$ (syst.). At the low energies 0-10 GeV where most of the expected neutrino oscillations occur, 451 events were observed compared to the unoscillated expectation of $686 \pm 39$ events, which shows a $5 \sigma$ deficit. Under the assumption the observed deficit is due to $\nu_{\mu} \rightarrow \nu_{\tau}$ oscillations, a fit is performed to extract the parameters $\left|\Delta m_{32}\right|^{2}$ and $\sin ^{2}\left(2 \theta_{23}\right)$ using the expression

$$
P\left(\nu_{\mu} \rightarrow \nu_{\mu}\right)=1-\sin ^{2}\left(2 \theta_{23}\right) \sin ^{2}\left(1.27 \Delta m_{32}^{2} \frac{L}{E}\right)
$$

where $L[\mathrm{~km}]$ is the distance from the target, $E[\mathrm{GeV}]$ is the neutrino energy, and $\left|\Delta m_{32}\right|^{2}$ is measured in $e V^{2} / c^{4}$.

The best fit to the neutrino energy spectrum yields $\left|\Delta m_{32}\right|^{2}=(2.43 \pm 0.13) \times$ $10^{-3} \mathrm{eV}^{2}$ and $\sin ^{2}\left(2 \theta_{23}\right)>0.95$ at $68 \%$ confidence level (C.L.) [59]. The fit includes the systematic uncertainties as nuisance parameters. The resulting $68 \%$ C.L. and $90 \%$ C.L. intervals for the oscillation parameters $\left|\Delta m_{32}\right|^{2}$ and $\sin ^{2}\left(2 \theta_{23}\right)$ are shown in 
Fig.1.8 (previous chapter).

\subsubsection{Search for Neutral Current Disappearance}

The MINOS experiment has reported a significant deficit of $\nu_{\mu}$ charged current (CC) interactions at the Far Detector relative to the non-oscillation prediction. If this deficit is due solely to conversions of $\nu_{\mu}$ to $\nu_{\tau}+\nu_{e}$, then the rate of neutral current interactions at the Far Detector would remain unchanged from the non-oscillation prediction. Alternatively, if any of the muon neutrinos convert to a sterile state, then the NC rate would be suppressed causing a distortion of the reconstructed energy spectrum. In the MINOS experiment, a search for evidence that muon neutrinos are oscillating to sterile neutrinos is performed by measuring the depletion of neutral current interactions at the Far Detector [109].

Based on an exposure of $2.46 \times 10^{20} \mathrm{POT}$, the depletion of the total neutral current event rate at the far site is limited to be below $17 \%$ at $90 \%$ confidence level without $\nu_{e}$ appearance. Assuming oscillations occur at a single mass-squared splitting, a fit to the neutral- and charged- current energy spectra shows the fraction of $\nu_{\mu}$ oscillating to a sterile neutrino is $0.28_{-0.28}^{+0.25}$ (stat. + syst.).

\subsubsection{Search for $\nu_{e}$ Appearance}

The MINOS experiment also has the potential to make the first measurement of the neutrino mixing angle $\theta_{13}$ by searching for a $\nu_{e}$ appearance signal in the Far Detector. This is the focus of my thesis. I will discuss the physics motivation of this measurement in the next chapter. 


\section{Chapter 3}

\section{MINOS $\nu_{\mu}$ to $\nu_{e}$ oscillation Search}

The neutrino mixing angle $\theta_{13}$ in the neutrino mixing matrix (1.93) is very important for understanding neutrino mixing. The value of this parameter has not been measured yet. The current best limit on this parameter was set by the reactor neutrino experiment CHOOZ: $\sin ^{2} 2 \theta_{13} \lesssim 0.15\left(\theta_{13} \lesssim 11.4^{\circ}\right)$ at $90 \%$ C.L. for $\Delta m^{2}=2.4 \times 10^{-3} \mathrm{eV}^{2}$ [61]. If $\theta_{13}$ is not zero, then we will ultimately be able to determine the ordering of the neutrino masses (normal versus inverted mass hierarchy) and measure CP violation in neutrino oscillations. There is theoretical speculation that $\mathrm{CP}$ violation by neutrinos could be one aspect of understanding why the universe is composed solely of matter, rather than equal amounts of matter and antimatter.

The MINOS experiment has the potential to constrain or make the first measurement of the neutrino mixing angle $\theta_{13}$ by searching for a $\nu_{e}$ appearance signal in the 
Far Detector. The 3-flavor $\nu_{\mu} \rightarrow \nu_{e}$ oscillation probability in vacuum is given by

$$
\begin{aligned}
P\left(\nu_{\mu} \rightarrow \nu_{e}\right) & =\left|\sum_{i=1}^{3} U_{\mu i}^{*} U_{e i} e^{-i \frac{m_{i}^{2} L}{2 E}}\right|^{2} \\
& =\left|U_{\mu 1}^{*} U_{e 1} e^{-i \frac{m_{1}^{2} L}{2 E}}+U_{\mu 2}^{*} U_{e 2} e^{-i \frac{m_{2}^{2} L}{2 E}}+U_{\mu 3}^{*} U_{e 3} e^{-i \frac{m_{3}^{2} L}{2 E}}\right|^{2} \\
& =\left|\left(-U_{\mu 2}^{*} U_{e 2}-U_{\mu 3}^{*} U_{e 3}\right) e^{-i \frac{m_{1}^{2} L}{2 E}}+U_{\mu 2}^{*} U_{e 2} e^{-i \frac{m_{2}^{2} L}{2 E}}+U_{\mu 3}^{*} U_{e 3} e^{-i \frac{m_{3}^{2} L}{2 E}}\right|^{2} \\
& =\left|2 U_{\mu 3}^{*} U_{e 3} e^{-i \Delta_{32}} \sin \Delta_{31}+2 U_{\mu 2}^{*} U_{e 2} \sin \Delta_{21}\right|^{2}
\end{aligned}
$$

where $\Delta_{i j} \equiv \frac{m_{i j}^{2} L}{4 E} \approx 1.27\left(\frac{\Delta m_{i j}^{2} L}{E}\right), \Delta m_{i j}$ is measured in $\mathrm{eV}^{2}, L$ is measured in $\mathrm{km}$, and $E$ is measured in $G e V$. Here we have used the unitary condition $U_{\alpha i}^{*} U_{\beta i}=\delta_{\alpha \beta}$. The last form of Eq.(3.1) is especially illuminating as the first term is the amplitude associated with the atmospheric $\Delta m^{2}$ and the second term is the amplitude associated with the solar $\Delta m^{2}$. Using the mixing matrix given in Eq.(1.93), we have

$$
\begin{aligned}
2 U_{\mu 3}^{*} U_{e 3} & =e^{-i \delta} \sin 2 \theta_{13} \sin \theta_{23} \\
2 U_{\mu 2}^{*} U_{e 2} & =\sin 2 \theta_{12} \cos \theta_{23} \cos \theta_{13}+\mathcal{O}\left(\sin \theta_{13}\right)
\end{aligned}
$$

where $\delta$ is the CP-violating phase (often referred to as $\left.\delta_{C P}\right)$. Since the $\mathcal{O}\left(\sin \theta_{13}\right)$ term is multiplied by $\sin \Delta_{21}$ in the amplitude, it is quadratic in the small quantities $\sin \theta_{13}$ and the solar $\Delta m^{2}$ and therefore can be neglected at the distance of the MINOS baseline. The oscillation probability can be written as

$$
\begin{aligned}
P\left(\nu_{\mu} \rightarrow \nu_{e}\right)= & \left|e^{-i\left(\Delta_{32}+\delta\right)} \sin 2 \theta_{13} \sin \theta_{23} \sin \Delta_{31}+\sin 2 \theta_{12} \cos \theta_{23} \cos \theta_{13} \sin \Delta_{21}\right|^{2} \\
= & \sin ^{2} \theta_{23} \sin ^{2} 2 \theta_{13} \sin ^{2} \Delta_{31}+\cos ^{2} \theta_{13} \cos ^{2} \theta_{23} \sin ^{2} 2 \theta_{12} \sin ^{2} \Delta_{21} \\
& +J_{r} \sin \Delta_{21} \sin \Delta_{31} \cos \left(\Delta_{32}+\delta\right) \\
= & P_{\text {atm }}+P_{\text {sol }}+P_{\text {int }}
\end{aligned}
$$


where $J_{r} \equiv \sin 2 \theta_{12} \sin 2 \theta_{23} \sin 2 \theta_{13} \cos \theta_{13}$ and $P_{a t m}, P_{\text {sol }}, P_{\text {int }}$ denote the atmospheric term, the solar term, and the interference term. Using the best-fit parameters in Table $1.2\left(\theta_{12}=33.9^{\circ}\right.$ and $\left.\theta_{23}=45^{\circ}\right), P_{a t m}, P_{\text {sol }}, P_{\text {int }}$ take the forms

$$
\begin{aligned}
P_{\text {atm }} & \approx 0.5 \sin ^{2} 2 \theta_{13} \sin ^{2} \Delta_{31} \\
P_{\text {sol }} & \approx 0.4 \sin ^{2} \Delta_{21} \\
P_{\text {int }} & \approx 0.9 \sin 2 \theta_{13} \sin \Delta_{21} \sin \Delta_{31} \cos \left(\Delta_{32}+\delta\right)
\end{aligned}
$$

Since $\Delta m_{21} \ll\left|\Delta m_{31}\right|$, the mass hierarchy ratio $\alpha \equiv \frac{\Delta m_{21}}{\Delta m_{31}}$ is small:

$$
|\alpha| \equiv \frac{\Delta m_{21}}{\left|\Delta m_{31}\right|} \approx 0.033,0.030 \lesssim|\alpha| \lesssim 0.037(1 \sigma)
$$

At the first oscillation maximum of the atmospheric $\Delta m^{2}$ scale, $\left|\Delta_{31}\right| \approx\left|\Delta_{32}\right|=\pi / 2$, $\Delta_{21}=|\alpha| \pi / 2, P_{\text {atm }}, P_{\text {sol }}, P_{\text {int }}$ become

$$
\begin{aligned}
P_{a t m} & \approx 0.5 \sin ^{2} 2 \theta_{13} \\
P_{\text {sol }} & \approx 0.001 \\
P_{\text {int }} & \approx-0.05 \sin 2 \theta_{13} \sin \delta
\end{aligned}
$$

Note that $P_{\text {int }}$ takes the '-' sign for both the normal mass hierarchy $\left(\Delta_{32}=\pi / 2\right)$ and the inverted mass hierarchy $\left(\Delta_{32}=-\pi / 2\right)$. As long as $\sin ^{2} 2 \theta_{13} \gg 0.002$ is satisfied, the contribution of the solar term to the oscillation probability is negligible compared with the atmospheric term. However, the interference term $P_{\text {int }}$ is proportional to $\sin 2 \theta_{13}$, while the leading term (atmospheric term) is proportional to $\sin ^{2} 2 \theta_{13}$. Thus, the relative importance of the sub-leading terms grows as $\sin ^{2} 2 \theta_{13}$ gets smaller.

Fig.3.1 shows the probability of $\nu_{\mu} \rightarrow \nu_{e}$ conversion from FNAL to Soudan for 
different $\delta_{C P}$ values assuming $\theta_{13}$ is at the $\mathrm{CHOOZ}$ bound. The distance between FNAL and Soudan is $L=735 \mathrm{~km}$. For a $1.4 \mathrm{GeV}$ neutrino energy (corresponding to the first atmospheric oscillation maximum), the sub-leading terms can give a maximal $25 \%$ enhancement or suppression in the transition probability if $\delta_{C P}$ differs from 0 .
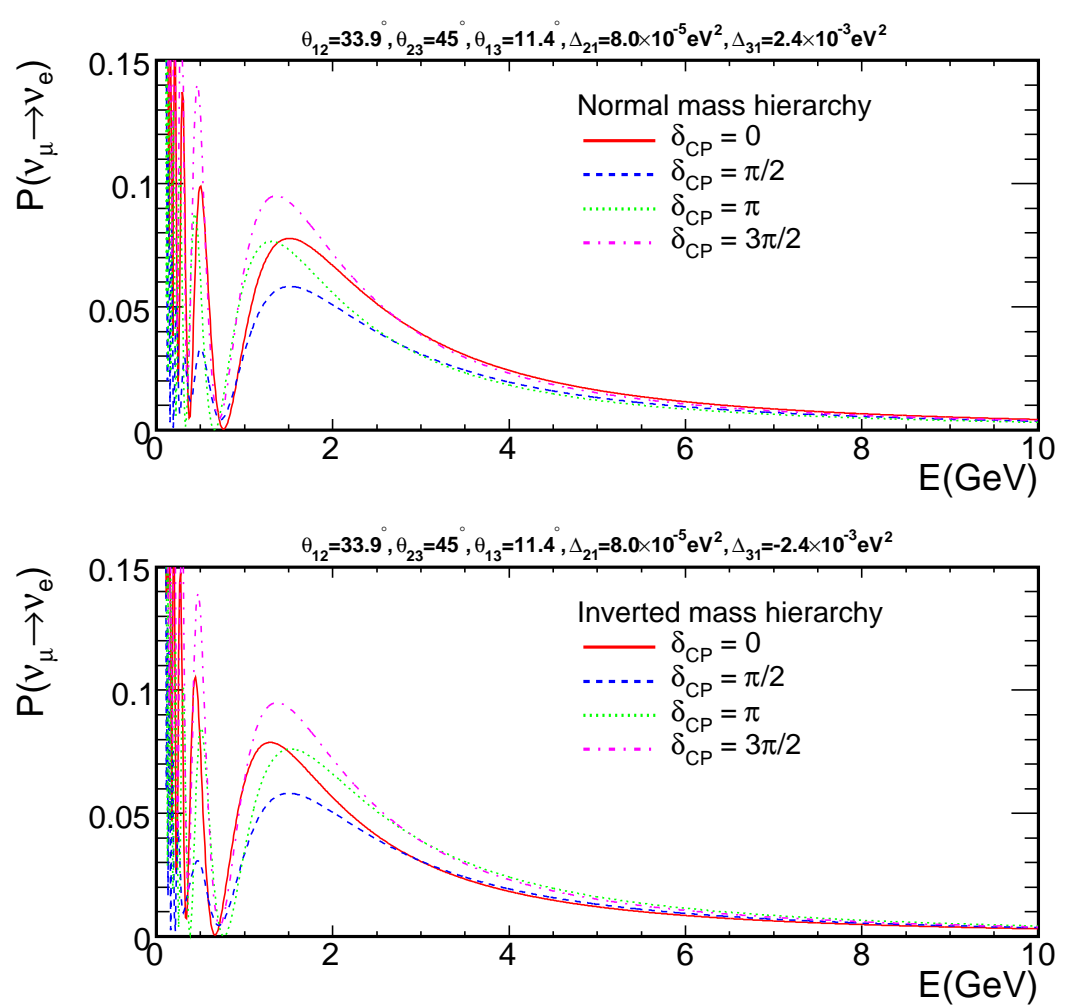

Figure 3.1: Probability of $\nu_{\mu} \rightarrow \nu_{e}$ conversion from FNAL to Soudan as a function of neutrino energy for different $\delta_{C P}$ values and for the normal neutrino mass hierarchy (top) and the inverted mass hierarchy (bottom).

The neutrinos in the NuMI beam propagate through the Earth and matter induced contributions to the propagation amplitude are non-negligible. These matter effects have opposite sign for neutrinos and anti-neutrinos and for the normal versus inverted neutrino mass hierarchies. In order to find the neutrino oscillation probabilities in the matter, one has to solve the Schorödinger equation for the neutrino vector of state in 
the flavor basis $|\nu(t)\rangle=\left(\left|\nu_{e}(t)\right\rangle\left|\nu_{\mu}(t)\right\rangle\left|\nu_{\tau}(t)\right\rangle\right)^{T}$ :

$$
\mathrm{i} \frac{\mathrm{d}}{\mathrm{dt}}|\nu(t)\rangle=H|\nu(t)\rangle
$$

with the effective Hamiltonian

$$
H \simeq \frac{1}{2 E} U \operatorname{diag}\left(0, \Delta m_{21}^{2}, \Delta m_{31}^{2}\right) U^{\dagger}+\operatorname{diag}(V, 0,0)
$$

Here $\mathrm{V}$ is the charged-current contribution to the matter-induced effective potential of $\nu_{e}[80,81,82]$ :

$$
V=\sqrt{2} G_{F} n_{e} \simeq 7.56 \times 10^{-14}\left(\frac{\rho(x)}{\mathrm{g} / \mathrm{cm}^{3}}\right) Y_{e}(x) \mathrm{eV},
$$

where $G_{F}$ is the Fermi coupling constant, $n_{e}$ is the number density of electrons, $\rho(x)$ is the matter density along the neutrino path and $Y_{e}(x)$ is the number of electrons per nucleon $(Z / A)$. For the matter of the Earth through which the neutrinos in the NuMI beam propagate, one has, to a very good accuracy, $\rho \simeq 2.8 \mathrm{~g} / \mathrm{cm}^{3}$ and $Y_{e} \simeq 0.5$.

The potential $V$ leads to an additional phase in the neutrino propagation: $\Delta \phi_{\text {matter }} \equiv$ $V t$, where $t$ is the time the neutrino traveled. The distance over which this "matter" phase equals $2 \pi$ defines the refraction length:

$$
l_{0} \equiv \frac{2 \pi}{V}=\frac{\sqrt{2} \pi}{G_{F} n_{e}}
$$

Unlike the vacuum oscillation length, $l_{\nu}=4 \pi E / \Delta m^{2}$, the refraction length does not depend on the neutrino energy.

The three-flavor neutrino oscillation probabilities in matter can be calculated by diagonalizing the effective Hamiltonian (3.12). The $\nu_{\mu} \rightarrow \nu_{e}$ conversion can be expanded in the mass hierarchy ratio $\alpha(3.7)$ and $\sin \theta_{13}$. The series expansion of the 
transition probability in matter of constant density up to second order in both $\alpha$ and $\sin \theta_{13}$ is [112]:

$$
\begin{aligned}
P\left(\nu_{\mu} \rightarrow \nu_{e}\right)= & \sin ^{2} 2 \theta_{13} \sin ^{2} \theta_{23} \frac{\sin ^{2}\left[(1-A) \Delta_{31}\right]}{(1-A)^{2}}+\alpha^{2} \cos ^{2} \theta_{13} \cos ^{2} \theta_{23} \sin ^{2} 2 \theta_{12} \frac{\sin ^{2}\left(A \Delta_{31}\right)}{A^{2}} \\
& +\alpha J_{r} \cos \left(\Delta_{31}+\delta\right) \frac{\sin \left(A \Delta_{31}\right)}{A} \frac{\sin \left[(1-A) \Delta_{31}\right]}{1-A}
\end{aligned}
$$

where

$$
\begin{aligned}
A & \equiv \frac{l_{0}}{l_{\nu}}=\frac{2 E V}{\left|\Delta m_{31}^{2}\right|}=\frac{E}{11 G e V} \frac{2.4 \times 10^{-3} \mathrm{eV}^{2}}{\left|\Delta m_{31}^{2}\right|} \\
J_{r} & \equiv \sin 2 \theta_{12} \sin 2 \theta_{23} \sin 2 \theta_{13} \cos \theta_{13}
\end{aligned}
$$

It is easy to verify that as $A$ approaches 0 (as is the case in vacuum), the transition probability in matter (3.15) becomes identical to the transition probability in vacuum $(3.3)$.

Fig.3.2 shows the probability of $\nu_{\mu} \rightarrow \nu_{e}$ conversion from FNAL to Soudan as a function of neutrino energy for different $\delta_{C P}$ values. Results are shown with and without the matter effects. For the normal hierarchy, matter effects enhance (suppress) the transition probability for neutrinos (anti-neutrinos) and vice versa for the inverted hierarchy. For a $1.4 \mathrm{GeV}$ neutrino energy, matter effects give a $25 \%$ enhancement or suppression in the transition probability.

The sensitivity of a $\nu_{\mu} \rightarrow \nu_{e}$ analysis depends on the separation of $\nu_{e} \mathrm{CC}$ events and $\pi^{0}$ background events. There are also a small number of intrinsic $\nu_{e}$ neutrinos in the NuMI beam originating from muon and kaon decays, which can obscure the presence of small $\nu_{\mu} \rightarrow \nu_{e}$ signals. There is additional source of contamination due to $\tau \rightarrow e$ decays in the Far Detector. Chapter 4 describes a neural network based method for $\nu_{e}$ identification.

The MC simulation plays a very important role in the $\nu_{\mu} \rightarrow \nu_{e}$ analysis. Chapter 

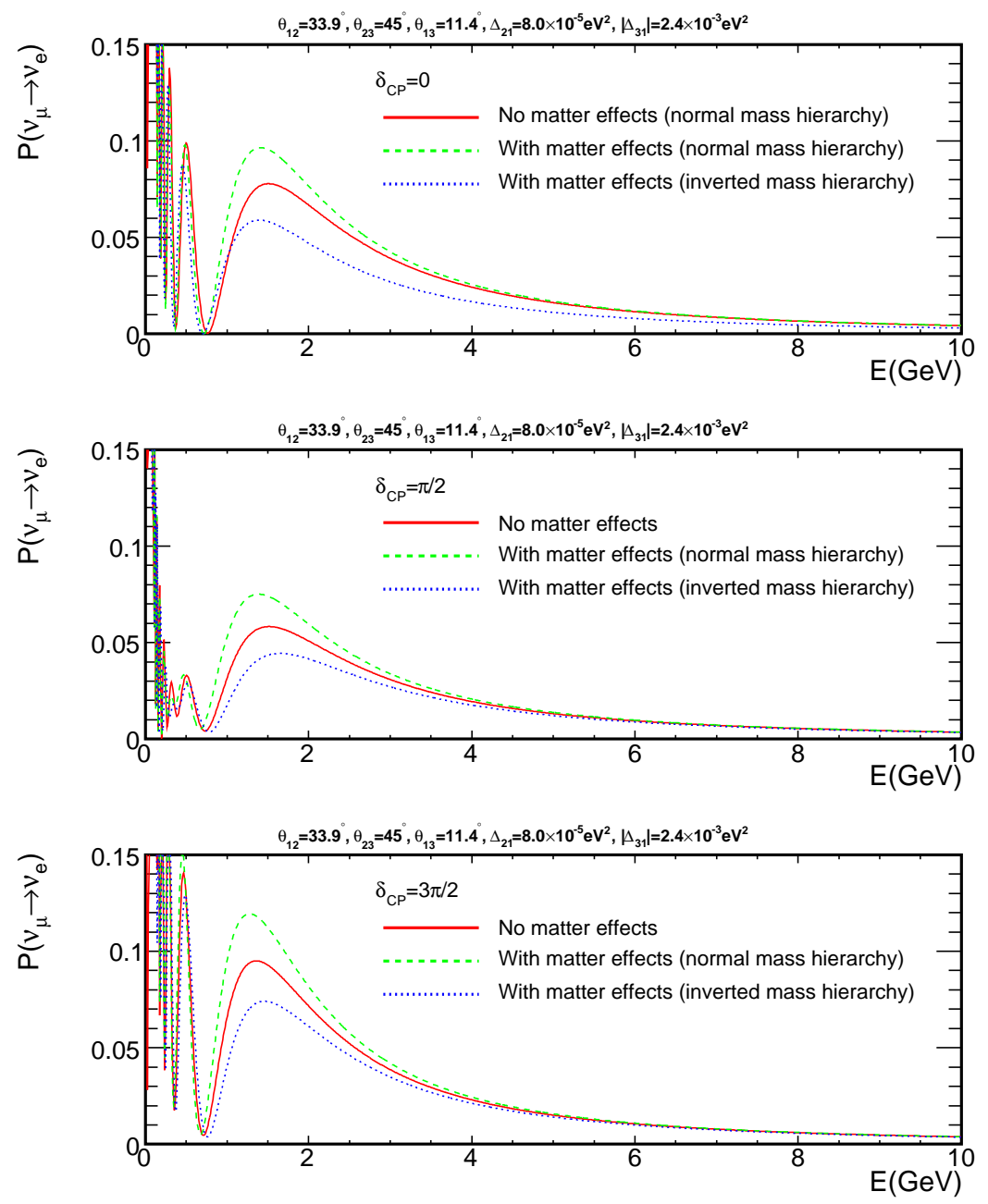

Figure 3.2: Probability of $\nu_{\mu} \rightarrow \nu_{e}$ conversion from FNAL to Soudan as a function of neutrino energy for different $\delta_{C P}$ values. Results are shown without the matter effects (solid) and with matter effects included (dashed and dotted). The dashed line (the upper line) assumes a normal mass hierarchy while the dotted line (the lower line) assumes a inverted mass hierarchy. 
5 is devoted to the discussion of hadronic multiparticle production in the neutrino interactions. An accurate modeling of the hadron species and energy distribution in the neutrino-nucleon interactions is important for the correct modeling of shower topology. We tuned the hadronization model using bubble chamber experimental data in order to improve the MC simulation.

It was discovered that the $\nu_{e}$ selection algorithm is quite sensitive to the low pulse height hits, especially the cross talk hits in the event. In Chapter 6, I will discuss the impact of the low pulse height hits to the $\nu_{e}$ analysis, the improvement of the cross talk simulations at both detectors, and how we can make the $\nu_{e}$ selection algorithm less sensitive to the low energy hits.

A limit on $\theta_{13}$ can be set by comparing the number of Far Detector $\nu_{e}$-like events with the MC prediction assuming $\theta_{13}=0$. Because the expected appearance signal is very small, precise understanding of background is crucial. We measure the background rate in the Near Detector and we predict the background rate in the Far Detector by extrapolating the Near Detector background rate. The advantage of the extrapolation is that many systematic errors cancel to a large extent since they are present at both detectors. However, the extrapolation to the Far Detector is complex for the $\nu_{\mu} \rightarrow \nu_{e}$ analysis because different background sources extrapolate differently. The $\nu_{\mu} \mathrm{CC}$ background is suppressed in the Far Detector because of $\nu_{\mu} \rightarrow \nu_{\tau}$ oscillation while the NC background remains unchanged. Thus some knowledge about the relative contribution from different background sources is necessary. Chapter 7 describes a method that can be used to obtain relative $\nu_{\mu} \mathrm{CC}$ and $\mathrm{NC}$ contributions from comparison of background rates in the horn-on and horn-off configurations. These two configurations give significantly different ratios of $\nu_{\mu} \mathrm{CC}$ to $\mathrm{NC}$ backgrounds and thus a comparison of background levels can yield information regarding relative contributions from these two sources.

The FD background predictions are mainly obtained through extrapolation. We 
discuss the FD background prediction, systematic errors, and MINOS sensitivity to $\theta_{13}$ in Chapter 8. We show the final results of the $\nu_{\mu} \rightarrow \nu_{e}$ oscillation analysis in Chapter 9. 


\section{Chapter 4}

\section{Electron Neutrino Identification}

The search for $\nu_{\mu} \rightarrow \nu_{e}$ signal is based on detection of charged current $\nu_{e}$ interactions in the MINOS Far Detector. The primary signature of a $\mathrm{CC} \nu_{e}$ interaction in the MINOS detectors is a shower consistent with the electromagnetic cascade in the absence of a long track. Thus it is very important to understand the differences between electromagnetic and hadronic showers in the MINOS detectors. In this chapter, we will first discuss the study done using the electron and pion data taken at the MINOS Calibration Detector (CalDet), focusing on the distinct characteristics of the electromagnetic showers. Then we will describe a neutral network based method for selecting electron neutrinos in the MINOS detectors.

\subsection{CalDet Electrons and Pions}

The CalDet was built to determine the response of MINOS detectors to electromagnetic and hadronic interactions in the few-GeV regime. During the years 2001-2003 CalDet was exposed to beams of $p^{+}, \pi^{ \pm}, e^{ \pm}, \mu^{ \pm}$between $0.4-10 \mathrm{GeV}$ at the CERN Proton Synchrotron (PS) T7 and T11 test beams. The CalDet has a mass of 12 tons and was composed of $601 \mathrm{~m} \times 1 \mathrm{~m}$ steel scintillator planes. Each scintillator plane 
contained 24 strips with orientations going from horizontal to vertical on alternating planes. Scintillator signals were transported to PMTs using the same WLS and clear fibers as in the Near and Far Detectors. To allow estimation of systematic differences between the two main detectors, CalDet ran with Far Detector electronics, Near Detector electronics and a hybrid mode where one side of the strips were read out using FD electronics and the other side using ND electronics. Time-of-flight and threshold Cerenkov Detectors were used to identify electrons, pions or muons, and protons. Muon/pion separation was accomplished by considering the event topology.

This section discusses the studies on the electromagnetic and hadronic event topologies using CalDet data. We will first show event displays of a typical $2 \mathrm{GeV} / \mathrm{c}$ electron and a typical $2 \mathrm{GeV} / \mathrm{c}$ pion to demonstrate the general features of the electromagnetic and hadronic showers in the MINOS detectors. Then we will examine the longitudinal and lateral distributions of the shower development. We will also compare our results based on the CalDet measurements with external calculations or simulations.

Fig.4.1 shows a typical $2 \mathrm{GeV} / \mathrm{c}$ electron and a typical $2 \mathrm{GeV} / \mathrm{c}$ pion in the CalDet. The $\mathrm{x}$ axis denotes the beam (longitudinal) direction and the $\mathrm{y}$ axis denotes the transverse direction in two views. The color represents the pulse height of hits which is in MIPs. The beam particles enter the detector at around strip number 11. The outlier hits are created through PMT crosstalk (a small fraction of light injected into one PMT pixel is leaking into the neighboring pixels). The following differences in shower development can be seen in the event displays:

- The longitudinal development of electron showers is more compressed; they reach shower maximum earlier and are somewhat shorter; the hadronic showers are more scattered and are usually longer. Frequently a pion will go through several planes determined by the pion interaction length before showering. 

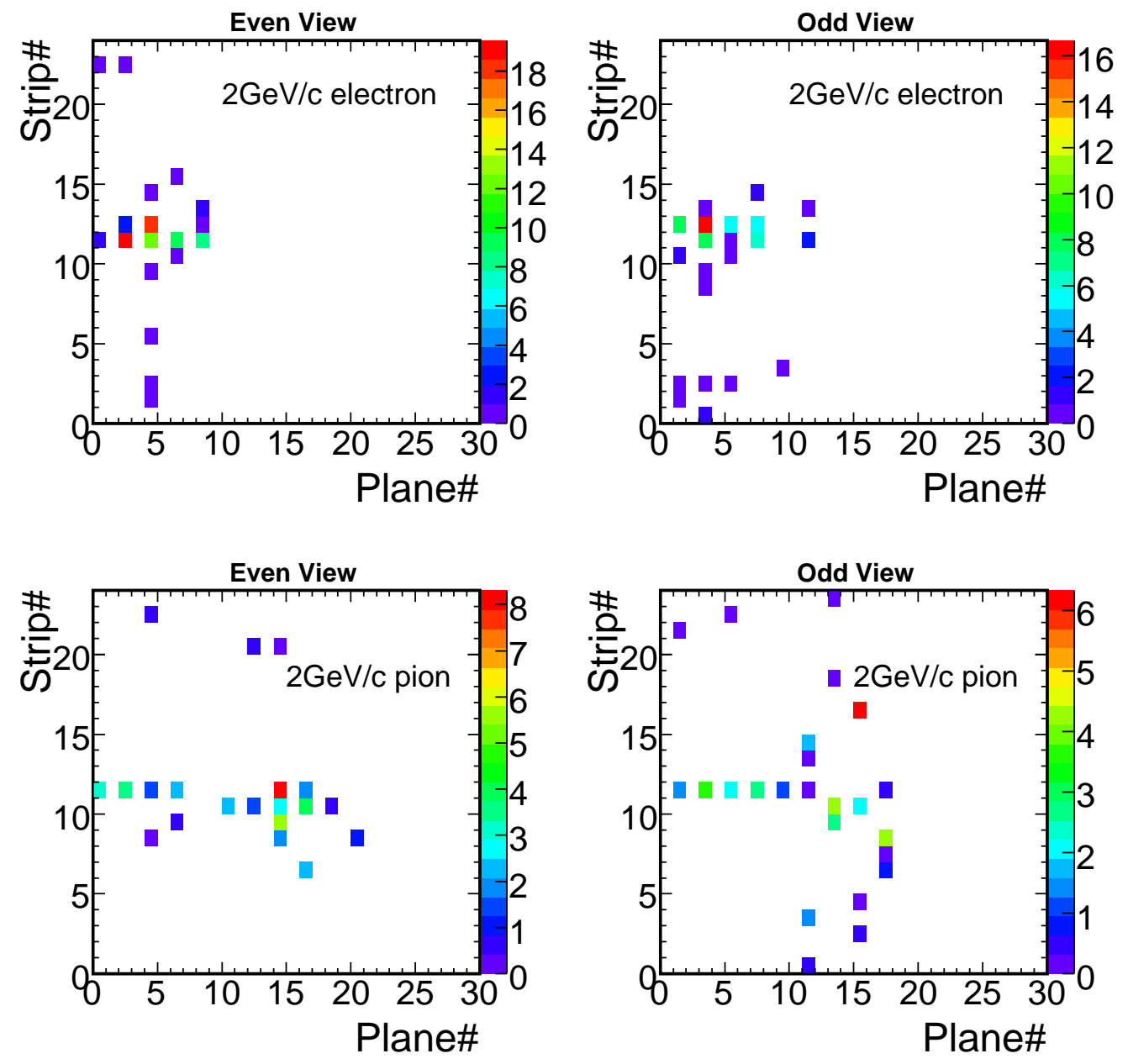

Figure 4.1: A typical $2 \mathrm{GeV} / \mathrm{c}$ electron (top 2 plots) and a typical $2 \mathrm{GeV} / \mathrm{c}$ pion (bottom 2 plots) in two views in the CalDet. The color scale represents the pulseheight which is in MIPs. 
- Electron showers are narrower than hadronic showers.

The above differences in the longitudinal and transverse shower characteristics are the basis of any methods that can separate the electromagnetic showers from the hadronic showers. High energy electrons can undergo bremsstrahlung process and emit high energy photons. The photons, in turn, materialize through $e^{+} e^{-}$pair production. It is through a succession of these energy loss mechanisms that the electromagnetic cascade is propagated, until the energy of the charged secondaries has been degraded to the regime dominated by ionization loss. Hadrons will initiate showers through their strong interactions with the nuclei in the absorber medium. The development of a hadronic shower is much more complex as there are many interactions which might occur, far more than the processes of bremsstrahlung and pair production which dominate electromagnetic shower formation. The strong interactions which produce the shower will give multiple particles with a typical transverse momentum of $350 \mathrm{MeV} / \mathrm{c}$, giving a shower with fairly large transverse dimensions together with a forward-going core of fast leading particles.

Now we take a close look at the longitudinal profiles of the shower development. Fig.4.2 shows the average energy deposition on each plane for $2 \mathrm{GeV} / \mathrm{c}$ electrons and pions. The error bar represents the spread (rms) of energy loss on each plane. It can be seen that the hadronic shower development has larger fluctuations. The depth of each plane is plotted in units of radiation length. The radiation length is defined as the distance over which a high-energy electron or position loses, on average, $63.2 \%$ (i.e. $1-e^{-1}$ ) of its energy by bremsstrahlung. It is also $7 / 9$ of the mean free path for $e^{+} e^{-}$pair production by a high-energy photon. It is usually measured in $\mathrm{g} / \mathrm{cm}^{2}$. The radiation length for a mixture of different materials can be calculated as follows

$$
\frac{1}{X_{0}}=\sum_{i} \frac{V_{i}}{X_{i}}
$$



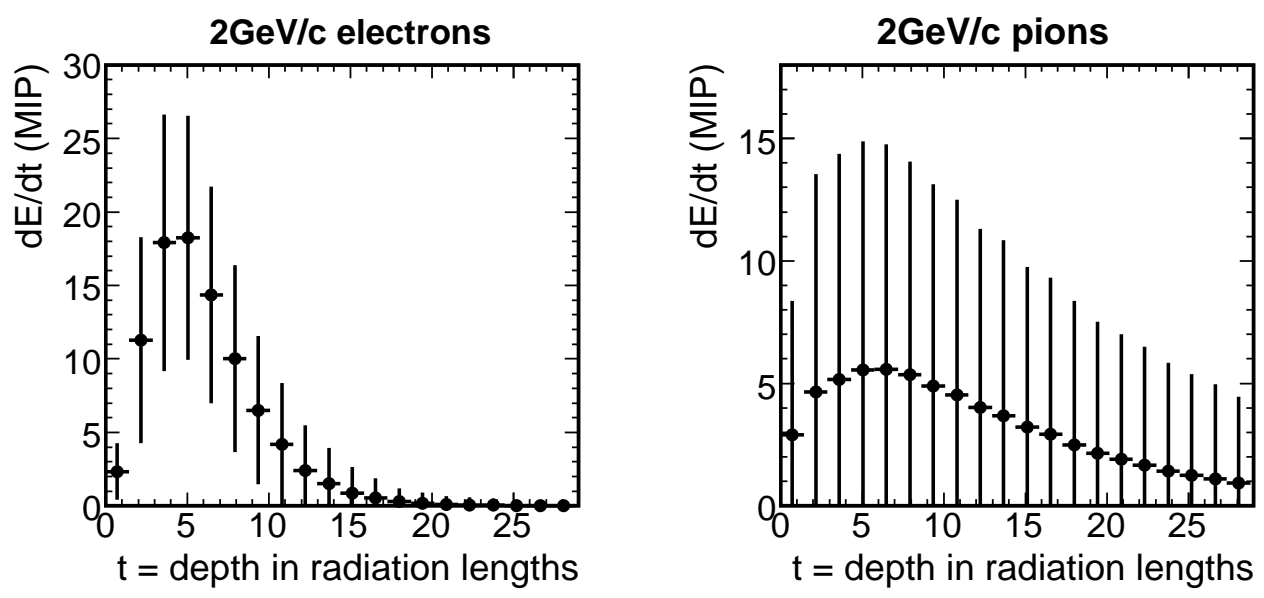

Figure 4.2: The average longitudinal shower profile for $2 \mathrm{GeV} / \mathrm{c}$ electrons and pions in CalDet. The error bar represents the spread (rms) of energy loss.

in which $V_{i}$ and $X_{i}$ are the fraction by volume and the radiation length (expressed in $\mathrm{cm}$ ) of the $i$ th component of the mixture. Eq.(4.1) may be used to calculate the effective radiation length of a calorimeter consisting of a variety of different materials. Let us consider the MINOS detectors, which are steel-scintillator sampling calorimeters. The steel and scintillator are arranged into a "sandwich" structure: a layer of $1 \mathrm{~cm}$ thick scintillator is attached to a layer of $2.54 \mathrm{~cm}$ thick steel plate to form a plane. The successive planes are separated by a $2.41 \mathrm{~cm}$ wide air-filled gap. The radiation lengths of steel, scintillator (polystyrene) and air are $1.76 \mathrm{~cm}, 47.9 \mathrm{~cm}$ and $3.04 \times 10^{4}$ $\mathrm{cm}$, respectively, and the fractional volume occupied by these elements is $42.7 \%$ for steel, $16.8 \%$ for scintillator and $40.5 \%$ for air. Therefore, we find the effective radiation length is: $X_{\text {eff }}=\left(0.427 / 1.76+0.168 / 47.9+0.405 / 3.04 \times 10^{4}\right)^{-1}=4.06 \mathrm{~cm}$. The distance between two successive planes is $5.95 \mathrm{~cm} \simeq 1.47$ radiation lengths.

Fig.4.3 shows the average longitudinal shower profile for electrons between 0.6 $\mathrm{GeV} / \mathrm{c}$ and $4 \mathrm{GeV} / \mathrm{c}$. The error bar represents the uncertainty on the mean energy deposition (calculated as spread $/ \sqrt{N}$, where $N$ is the number of data events obtained in each bin), which is negligible. The mean longitudinal profile of the energy 
deposition in an electromagnetic cascade is reasonably well described by a gamma distribution [113]:

$$
\frac{d E}{d t}=E_{0} b \frac{(b t)^{a-1} e^{-b t}}{\Gamma(a)}
$$

where $E_{0}$ is the total energy deposited, parameter $a$ describes the rise of the profile while parameter $b$ describes the tail of the profile, $t$ is the depth in radiation lengths. Both $E$ and $E_{0}$ are measured in MIPs. The results of the fits are shown in Fig.(4.3). The errors represent statistical uncertainties.

Fig.4.4 shows the best fit parameters $E_{0}, a$ and $b$ as a function of electron energy. These parameters can be parameterized as follows:

$$
\begin{aligned}
E_{0}(\mathrm{MIP}) & =-0.5+66.4 \times E_{e l} \\
a & =2.9+0.51 \times \ln \left(E_{e l}\right) \\
b & =0.55-0.002 \times E_{\text {el }}
\end{aligned}
$$

where the electron energy $E_{e l}$ is measured in $\mathrm{GeV}$.

Eq.(4.3) describes the detector response to electrons and is consistent with the measurement in [97]:

$$
E_{0}(\mathrm{MIP})=-1.46+65.8 \times E_{e l} .
$$

The small negative offset -0.5 can be interpreted as the upstream energy loss of the electron beams.

According to Eq.(4.2), the maximum shower development occurs at $t_{\max }=(a-$ $1) / b$. If we ignore the small energy dependence of parameter $b$ and assume $b \simeq 0.54$, $t_{\max }$ can be written as

$$
t_{\max }=(a-1) / b=3.5+0.94 \times \ln \left(E_{e l}\right) .
$$



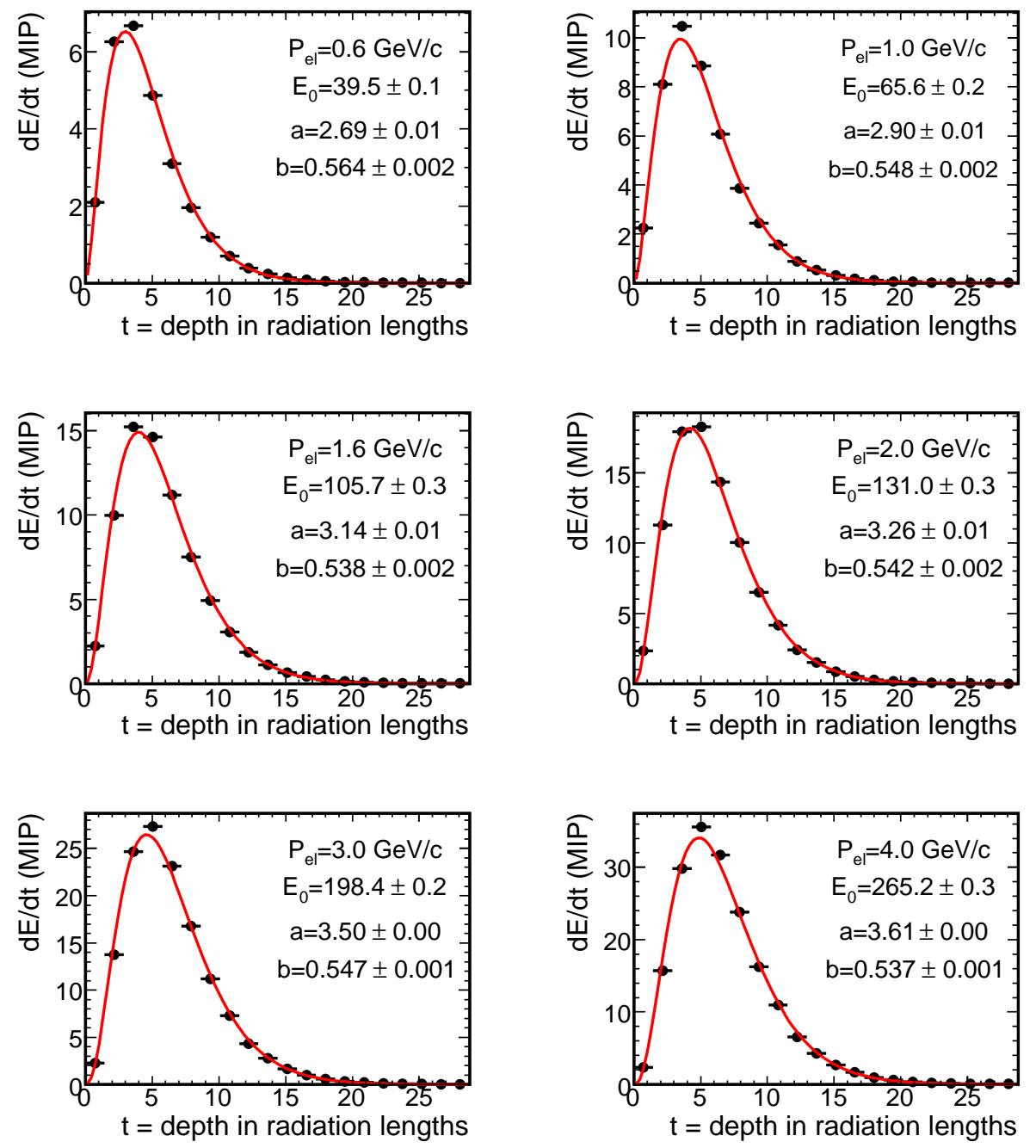

Figure 4.3: The average longitudinal shower profile for electrons in CalDet with various momenta. 

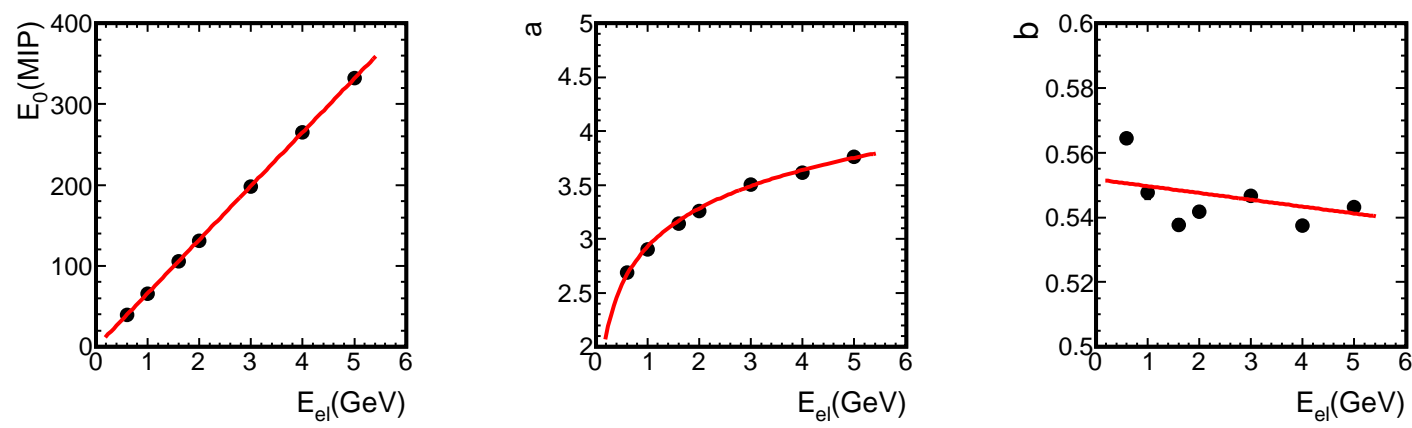

Figure 4.4: Best fit parameters $E_{0}, a$ and $b$ as a function of electron energy.

It is convenient to introduce the scale variables $y=E_{e l} / E_{c}$ where $E_{c}$ is the critical energy. $E_{c}$ is defined as the electron energy at which the average energy loss due to radiation equals that due to ionization and is given by [114]

$$
E_{c}(\mathrm{MeV})=\frac{610}{Z+1.24}
$$

for solid, where $\mathrm{Z}$ is the atomic number of the target material. For steel, $E_{c} \simeq$ $22.4 \mathrm{MeV}$. Thus $t_{\max }$ now takes the form

$$
t_{\max }=0.94 \times \ln (y)-0.07 .
$$

A similar form was obtained by Rossi in the context of his "Approximation B" [115]:

$$
t_{\max }=1.0 \times \ln (y)-1.0 .
$$

Another form was obtained using a simulation program EGS4 [114, 116]:

$$
t_{\max }=1.0 \times \ln (y)-0.5
$$

The comparison of our result with these forms is shown in Fig.4.5. Our result is close 
to the prediction of EGS4, which is a modern simulation program.

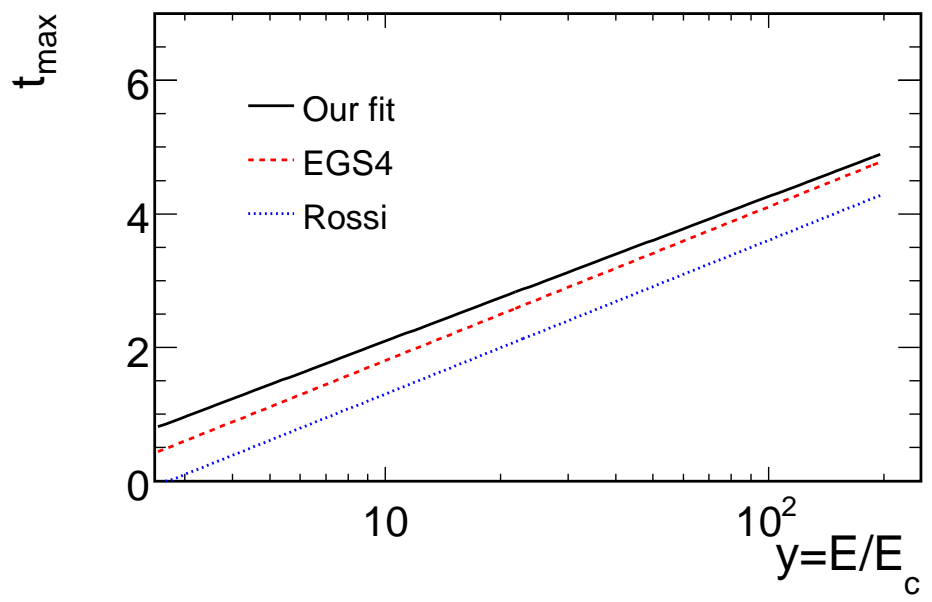

Figure 4.5: Maximum shower development $t_{\max }$ as a function of $y=E / E_{c}$.

Now we examine the lateral distributions of the shower development. Fig.4.6 shows the fraction of energy loss as a function of the transverse position for $2 \mathrm{GeV} / \mathrm{c}$ electrons and pions. The error bar represents the spread (rms) of the fractional energy loss. The lateral granularity of the MINOS calorimeter is $4.1 \mathrm{~cm}$ which is the scintillator strip width. It can be seen in the two plots that the electromagnetic showers are narrower than the hadronic showers. The electrons lose most of their energy within 2-3 strips while the pions manifest large radial spread.

Fig.4.7 shows the fraction of energy loss as a function of the transverse position for electrons between $0.6 \mathrm{GeV} / \mathrm{c}$ and $4 \mathrm{GeV} / \mathrm{c}$. The error bar represents the statistical uncertainty on the mean fractional energy loss. The distributions are fitted to a gaussian function and the width $(\sigma)$ from the gaussian fits is shown in the plots. Fig. 4.8 shows the average shower width as a function of the electron energy. It shows a small energy dependence:

$$
\sigma=3.15-0.06 \times E(G e V)
$$



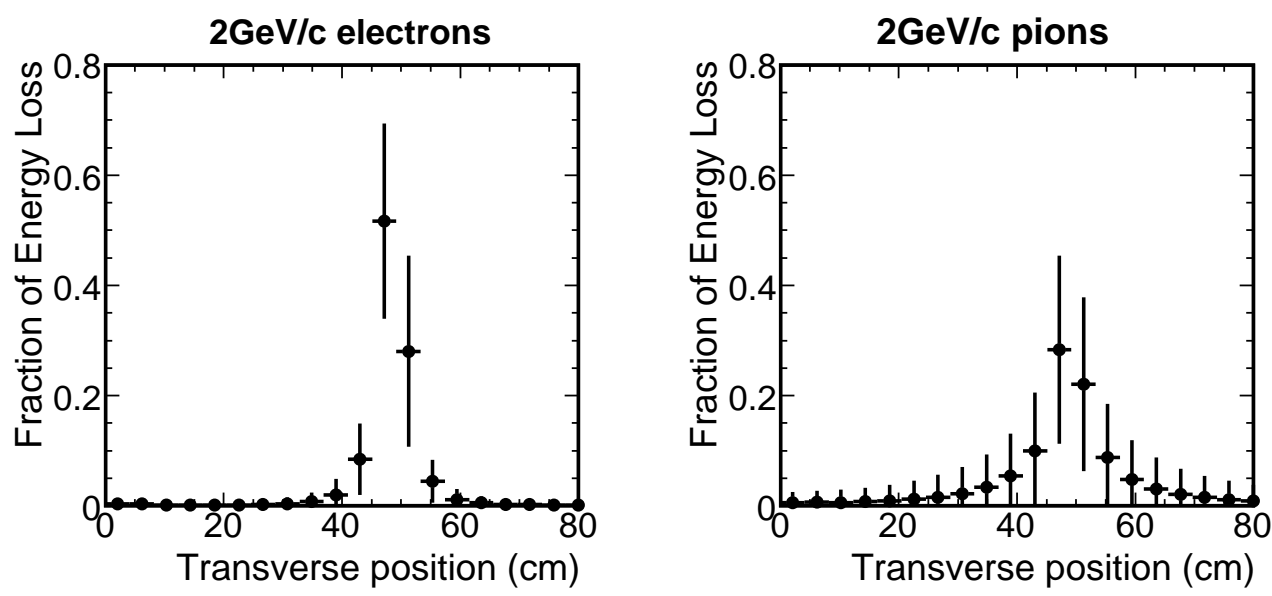

Figure 4.6: Fraction of energy loss as a function of the transverse position for 2 $\mathrm{GeV} / \mathrm{c}$ electrons and pions in CalDet. The error bar represents the spread (rms) of the fractional energy loss.

which means that as the electron energy increases, the electromagnetic shower becomes narrower.

The transverse development of electromagnetic showers in different materials scales fairly accurately with the Molière radius $R_{M}$. It is defined in terms of the radiation length $X_{0}$ and the critical energy $E_{c}$, as follows:

$$
R_{M}=X_{0} E_{s} / E_{c}
$$

where $E_{s} \equiv m_{e} c^{2} \sqrt{4 \pi / \alpha}=21.2 \mathrm{MeV}$ is the scale energy. On average, $90 \%$ of the shower energy is deposited in a cylinder with radius $R_{M}$ around the shower axis. The Molière radii of mixtures of different elements may be calculated in the same way as the radiation length:

$$
\frac{1}{R_{M}}=\frac{1}{E_{s}} \sum_{i} \frac{V_{i} E_{c i}}{X_{i}}
$$

in which $V_{i}, X_{i}$ and $E_{c i}$ are the fraction by volume, the radiation length (expressed in $\mathrm{cm}$ ) and the critical energy (expressed in $\mathrm{MeV}$ ) of the $i$ th component of the mixture. 

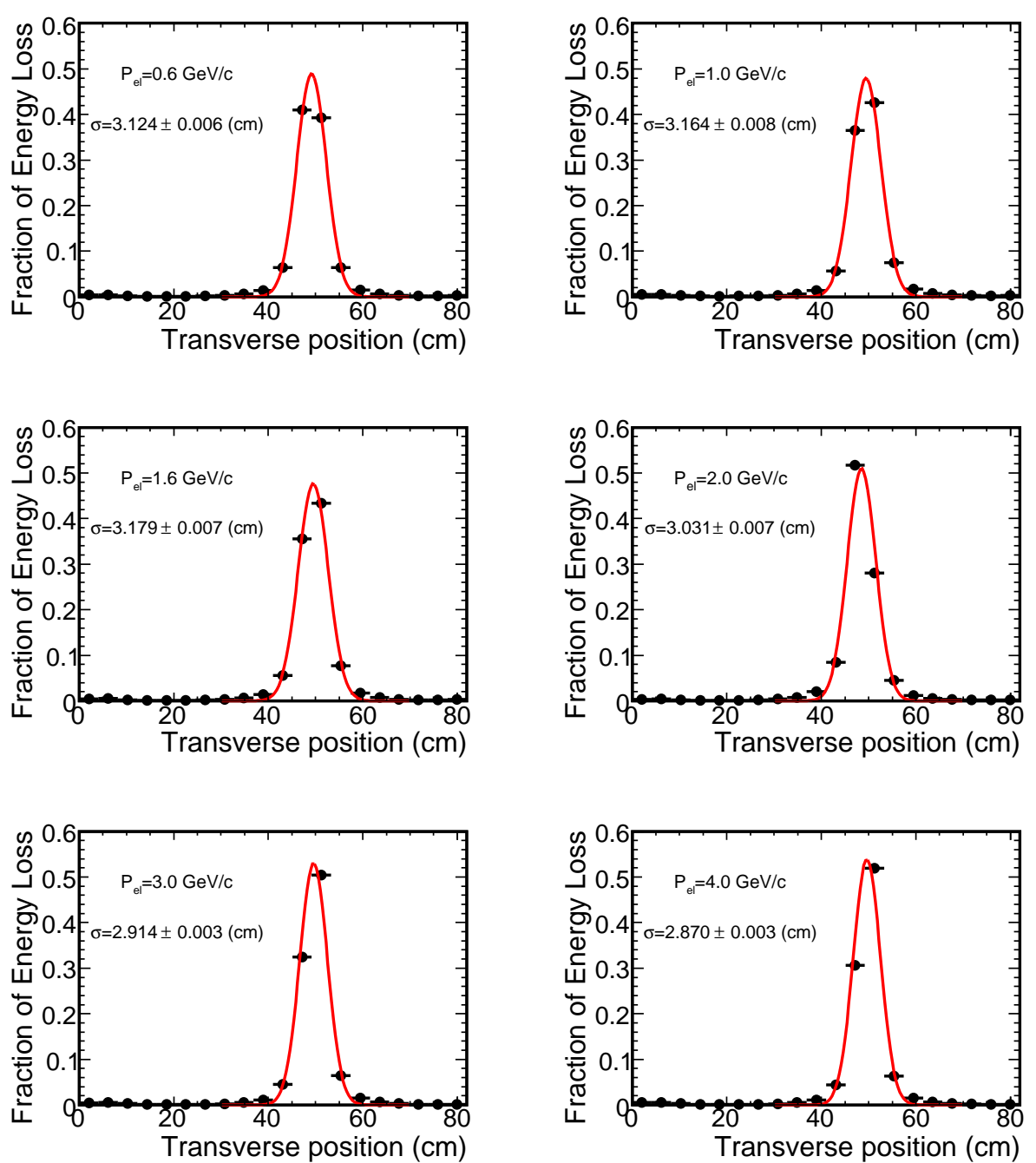

Figure 4.7: Fraction of energy loss as a function of the transverse position for electrons in CalDet with various momenta. 


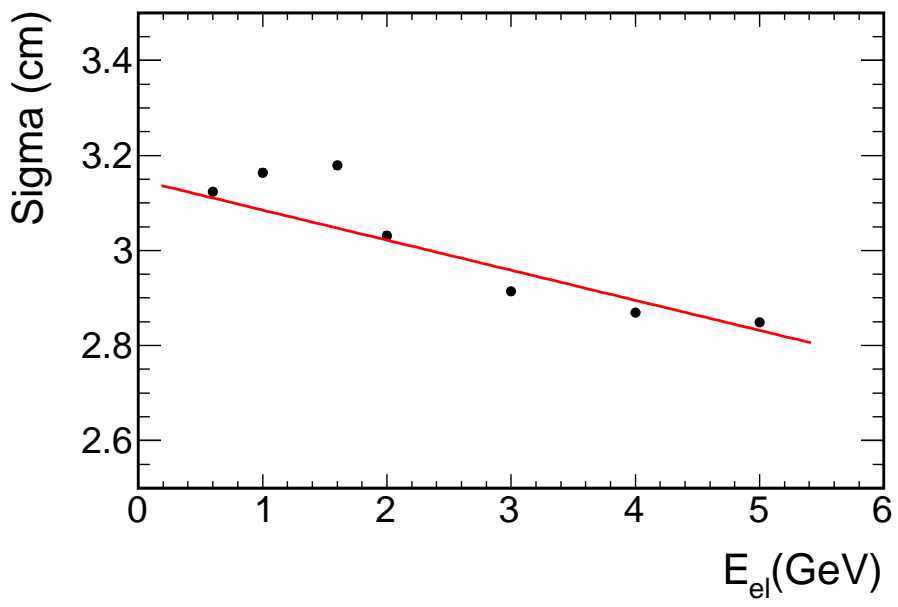

Figure 4.8: Average shower width as a function of the electron energy.

The effective Molière radius of the MINOS calorimeter is $3.7 \mathrm{~cm}$, which is of the same order as the shower width we measured in Eq.(4.12).

In summary, we have examined the properties of EM showers using CalDet data and verified the measured EM shower properties agree with external measurements and theoretical expectation. We also demonstrated the major topological differences between the electromagnetic and hadronic showers, i.e. the EM showers are generally compact and narrow while the hadronic showers are often more scattered and have larger fluctuations. In the next section, we will describe a neural network based $\nu_{e}$ identification method that takes into account the differences between the electromagnetic and hadronic showers.

\subsection{MINOS Electron Neutrino Identification}

\subsubsection{Overview}

The sensitivity of the MINOS $\nu_{\mu} \rightarrow \nu_{e}$ analysis depends on the separation of $\nu_{e}$ CC events and background events. The task of the $\nu_{e}$ identification algorithm is to look for 
the presence of an electron in the final state of the neutrino interactions, taking into account the differences between EM showers and hadronic showers described in the previous section. However, the $\nu_{e}$ identification is more difficult than the separation of electrons and charged mesons. The electron neutrino can create a bunch of hadrons by exchanging a $W$ boson with the target nucleon. The neutrino itself turns into an electron in this process. Depending on the kinematics of the interaction, sometimes the energy of the hadrons can be much larger than the energy of the electron, in which case it is almost impossible to identify the electron. Moreover, because of the limited granularity of the MINOS calorimeter, it is difficult to separate $\pi^{0}$ 's and electrons. The dominant background in the $\nu_{e}$ analysis is the $\pi^{0}$ produced via NC interaction. Other background sources consist of $\nu_{\mu} \mathrm{CC}$ interaction with a short muon track and $\pi^{0}$ 's, and the intrinsic $\nu_{e}$ component in the $\nu_{\mu}$ neutrino beam. In the Far Detector, there is additional background source: $\nu_{\tau}$ from $\nu_{\mu} \rightarrow \nu_{\tau}$ oscillations followed by $\tau \rightarrow e / \pi^{0}$ decays.

The strategy of selecting $\nu_{e} \mathrm{CC}$ events inside the detector can be summarized as follows: first we apply some pre-selection cuts to remove events that are obvious background events; then we compute several variables that describe the event topology and feed them into a neural network to enhance the signal/background separation.

We only expect to see potential $\nu_{\mu} \rightarrow \nu_{e}$ oscillation signals in the Far Detector, thus we tune the neural network based on the Far Detector MC. Throughout this section, we use the following oscillation parameters:

$$
\sin ^{2}\left(2 \theta_{13}\right)=0.15 \text { (CHOOZ's bound), }\left|\Delta m_{31}^{2}\right|=2.4 \times 10^{3} \mathrm{eV}^{2}, \sin ^{2}\left(2 \theta_{23}\right)=1 \text {. }
$$

We ignore the solar terms, terms the $\mathrm{CP}$ violating phases, and the matter effects in calculating the oscillation probabilities. We assume an exposure of 3.25 protons on target (POTs) which corresponds to roughly 2 years of running. 
We are only interested in the neutrino interactions that occur inside the detector and away from the magnet coil hole. Thus we apply the fiducial volume cuts to remove events that occur in the periphery of the detector or close to the coil hole. The Far Detector fiducial volume cuts we use in the $\nu_{e}$ analysis are:

$$
0.5 \leq \sqrt{x^{2}+y^{2}} \leq \sqrt{14}, 0.48 \leq z \leq 14.28 \text { or } 16.26 \leq z \leq 27.97
$$

where $(\mathrm{x}, \mathrm{y}, \mathrm{z})$ are the coordinates of the reconstructed event vertex, and $\mathrm{z}$ axis is along the beam direction. The lower radial cut is to exclude the coil hole. The two intervals of $\mathrm{z}$ cut correspond to the two super-modules.

\subsubsection{Pre-selection Cuts}

Pre-selection cuts: 1) reject events with a long track (predominantly $\nu_{\mu} \mathrm{CC}$ backgrounds); 2) reject events in which the visible energy does not fall with in the range of interest.
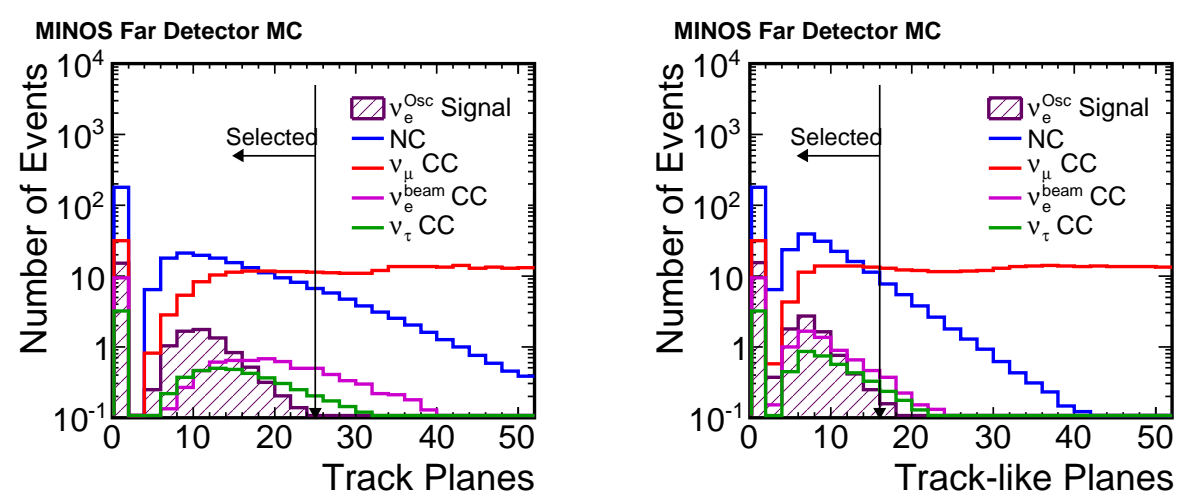

Figure 4.9: FD Track planes and track-like planes distributions after fiducial volume cuts.

Fig.4.9 shows the track length in number of planes that the track traverses for events in the fiducial volume. Track-like planes refer to the "clean" hit planes in the 
track, a clean plane is one in which the plane is free from the presence of non-track associated hits. The signal events usually have no reconstructed track or a very short track. A large number of $\nu_{\mu} \mathrm{CC}$ events have a long track from the muon. We remove those $\nu_{\mu} \mathrm{CC}$ background events by only accepting events satisfying the two track length cuts:

- Track planes $<25$, Track-like planes $<16$

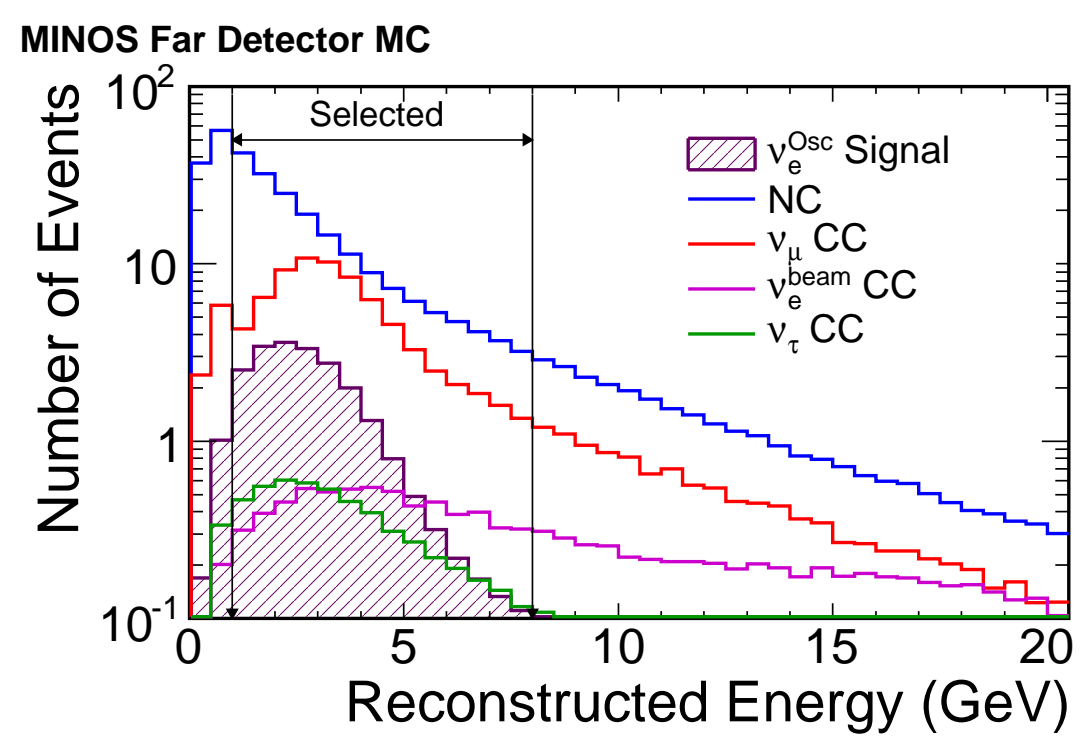

Figure 4.10: FD Reconstructed energy distributions after fiducial volume cuts and track length cuts.

Fig.4.10 shows the reconstructed energy distributions for events passing the fiducial volume cuts and the track length cuts. After the track length cuts, the NC events become the dominant background. We apply the following energy cut to further reduce background events:

- $1<E_{\text {reco }}<8 \mathrm{GeV}$

The $\nu_{\mu} \rightarrow \nu_{e}$ oscillations are highly suppressed at high energies. Thus we remove all events above $8 \mathrm{GeV}$. Note the beam $\nu_{e}$ distribution has a high energy tail. These 
high energy beam $\nu_{e}$ events are mostly from kaon decays. Since they are real electron neutrinos, the high energy cut is the only effective way of rejecting them. A lot of $\mathrm{NC}$ events have visible energy below $1 \mathrm{GeV}$ while only a small fraction of signal events have such low energy, thus we remove all events below $1 \mathrm{GeV}$.

There are several other cuts we apply in addition to the cuts described above. One cut requires that there should be at least one hit on each of 5 contiguous planes (these 5 planes can be anywhere inside the event). This cut aims to remove poorly reconstructed events. Furthermore, the pre-selection only accepts events which have more than zero showers. In the Far Detector, additional cuts are applied to remove cosmic ray background. After the pre-selection cuts, the signal over background ratio improves from 1:55 to $1: 12$ if $\theta_{13}$ is at $\mathrm{CHOOZ}$ limit. At this stage, most events only consist of one well reconstructed shower. The next step is to compute some topological variables and feed them into a neural network to further enhance the signal and background separation.

\subsubsection{PID Variables}

Over the past few years, a lot of effort within MINOS collaboration has been devoted to understanding the topological differences between the signal and background events and calculating quantities that can be used in the particle identification (PID) algorithms. In this section we describe several variables computed with FD MC for $\nu_{e}$ selection. Fig.4.11 shows the distributions of 11 variables that describe the longitudinal and lateral shower characteristics. In computing these variables, we use only hits whose pulse-heights are greater than $2 \mathrm{PE}$ in order to eliminate the dependency of the $\nu_{e}$ selection algorithm on the low pulse-height hits, in particular, the crosstalk hits. The justification of this $2 \mathrm{PE}$ cut will be discussed in detail in Chapter 6 . The meanings of the 11 variables are described as follows: 

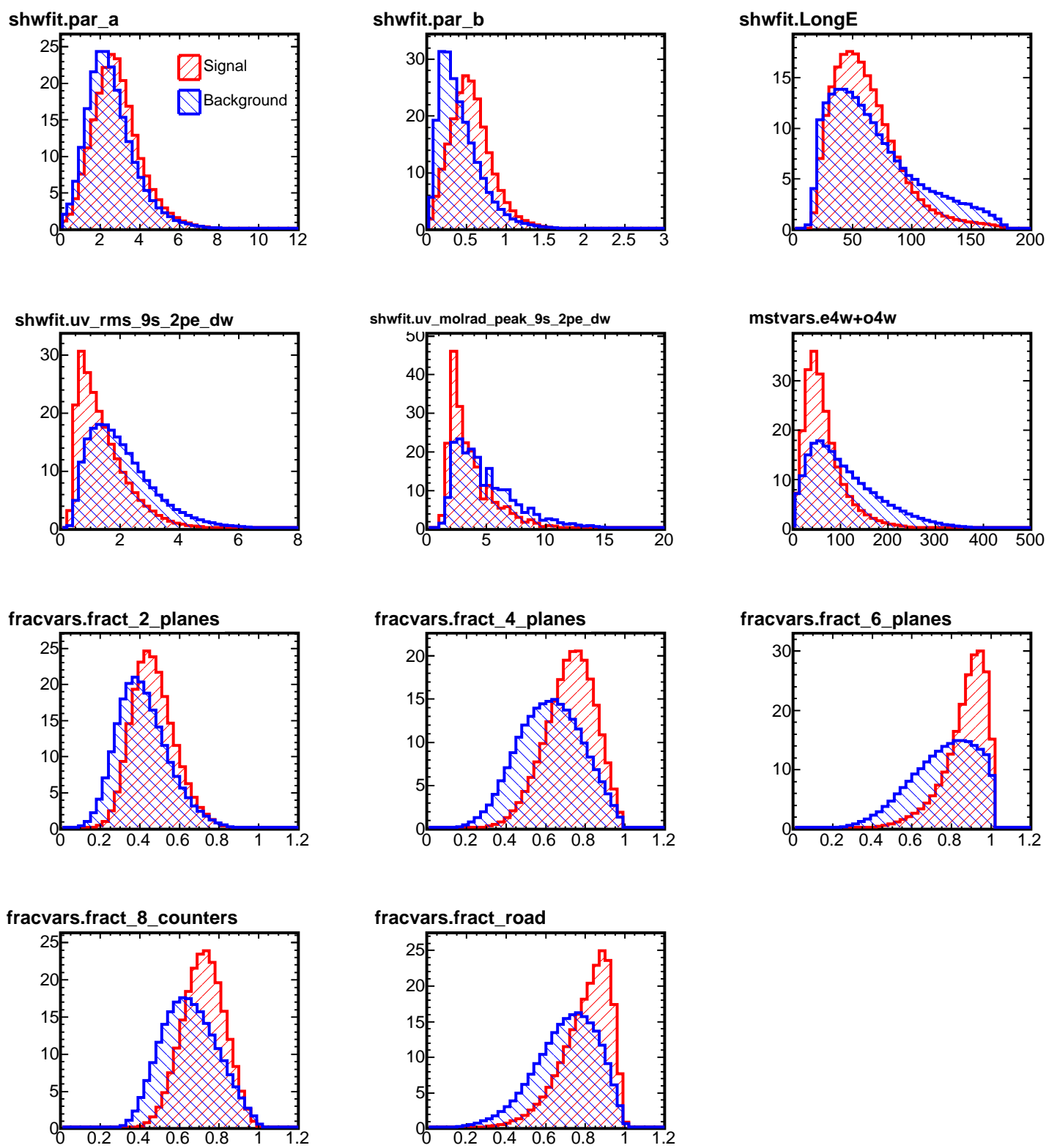

Figure 4.11: Distributions of the 11 variables used in the artificial neural network. Distributions are area normalized and only events passing the pre-selection cuts are used in making these plots. 
- shwfit.par_a, shwfit.par_b We fit the longitudinal profile of the energy loss of an event to a gamma function Eq.(4.2). These two variables are the best fit parameters $a$ and $b$. Parameter $a$ describes the rise of the longitudinal shower profile while parameter $b$ describes the tail of the profile. A small $b$ value means the shower profile has a long tail. It can be seen from the first 2 plots in Fig.4.11 that parameter $a$ is less discriminative than parameter $b$. We show parameter $a$ just for completeness. For $\nu_{e}$ events, parameter $a$ peaks at around 3 and parameter $b$ peaks at around 0.5 . These values are consistent with our CalDet measurements described in the previous section.

- shwfit.LongE This is defined as the sum of the magnitudes of the longitudinal projections of the vectors defined by each hit and the vertex. Each vector is multiplied by the hit pulse height.

- shwfit.uv_rms_9s_2pe_dw This variable is the RMS of the transverse energy loss profile of a shower. It describes how narrow a shower is. "9s_2pe_dw" means we ignore hits that are more than 9 strips away from the event vertex transversely and weight each strip by pulse-height squared instead of pulseheight to downgrade the importance of the low pulse-height hits. The reason is to make this variable less sensitive to the crosstalk hits.

- shwfit.uv_molrad_peak_9s_2pe__dw This variable is the radius (in the number of strips) of an imaginary cylinder around the shower axis which contains $90 \%$ of the visible event energy (Molière radius). "9s_2pe_dw" has the same meaning as in the variable shwfit.uv_rms_9s_2pe_dw. 
- mstvars.e4w+o4w We form a Minimal Spanning Tree ${ }^{1}$ from hits whose pulse height is larger than the average pulse height. This variable is the sum of distances between hits in the Minimal Spanning Tree.

- fracvars.fract_n_planes $(\mathbf{n = 2}, \mathbf{4}, \mathbf{6})$ We define a $\mathbf{n}$ plane sliding window and move the window through the event and find the maximum energy loss in the $\mathbf{n}$ plane window. This variable is the ratio of the maximum energy loss in the $\mathbf{n}$ plane sliding window over the total event energy. It describes how condensed the energy deposition is longitudinally. The $\nu_{e}$ events tend to have larger ratios of energy loss in the sliding window.

- fracvars.fract_8_counters We find the 8 strips in an event with the maximum energy deposition. This variable is the ratio of the total energy in the 8 strips over the total event energy. If an event has less than 8 strips, we assign 1 to this variable. This variable describes how likely the particles lose a lot of energy in a few strips.

- fracvars.fract_road We find the shower axis by doing a energy weighted least squares fit to the position of the shower strips. Then we identify all the strips whose center is located within 1.5 strip widths with respect to the shower axis. This variable is defined as ratio of the total energy in those strips over the event energy. This is found to be one of the most powerful variables. Because the EM shower is generally narrower than the hadronic shower, this ratio is peaked towards one for the signal events. In constrast, this ratio for $\mathrm{NC}$ and $\nu_{\mu} \mathrm{CC}$ background events gives a much broader distribution.

\footnotetext{
${ }^{1}$ Given a connected, undirected graph, a spanning tree of that graph is a subgraph which is a tree and connects all the vertices together. We can also assign a weight to each edge, which is a number representing how unfavorable it is. A minimum spanning tree or minimum weight spanning tree is then a spanning tree with weight less than or equal to the weight of every other spanning tree.
} 
These 11 variables are used to construct a neural network for the $\nu_{e}$ identification, which is discussed in the next section. We investigated the effect of adding more input variables to the neural network but the additional variables did not improve its performance (signal/background separation, robustness, etc.). Thus we decided to use only the 11 variables described in this section.

\subsubsection{Constructing ANN}

There is a growing interest in modern high energy physics experiments in the use of algorithms based on artificial neural networks (ANN) and related methods in order to perform data analyses. An ANN is composed of a large number of highly interconnected processing elements (neurones) working in unison to solve specific problems. It is configured for a specific application, such as pattern recognition or data classification, through a learning process. Learning involves adjustments to the synaptic connections that exist between the neurones.

TMultiLayerPerceptron is a ROOT implementation of ANN. It is a simple feedforward ${ }^{2}$ network based on the MLPfit package. A typical structure of this ANN is illustrated in Figure 4.12. We employ this ROOT class to build a neural network for the electron neutrino identification. For each event we compute 11 variables as described in the previous section and use them as the input to the ANN. We use $\nu_{e}$ $\mathrm{CC}$ events that pass the $\nu_{e}$ pre-selection cuts as the signal sample while we use the $\mathrm{NC}$ and $\nu_{\mu} \mathrm{CC}$ events that pass the $\nu_{e}$ pre-selection cuts as the background sample. Both the signal and the background events are weighted based on the neutrino oscillation probabilities. The training sample is split automatically into two independent samples, one sample for tuning the network weights, the other sample to check the performance of the network after each round of tuning in order to avoid overtraining.

\footnotetext{
${ }^{2}$ Feed-forward ANNs allow signals to travel one way only, from input to output. There is no feedback (loops) i.e. the output of any layer does not affect that same layer.
} 


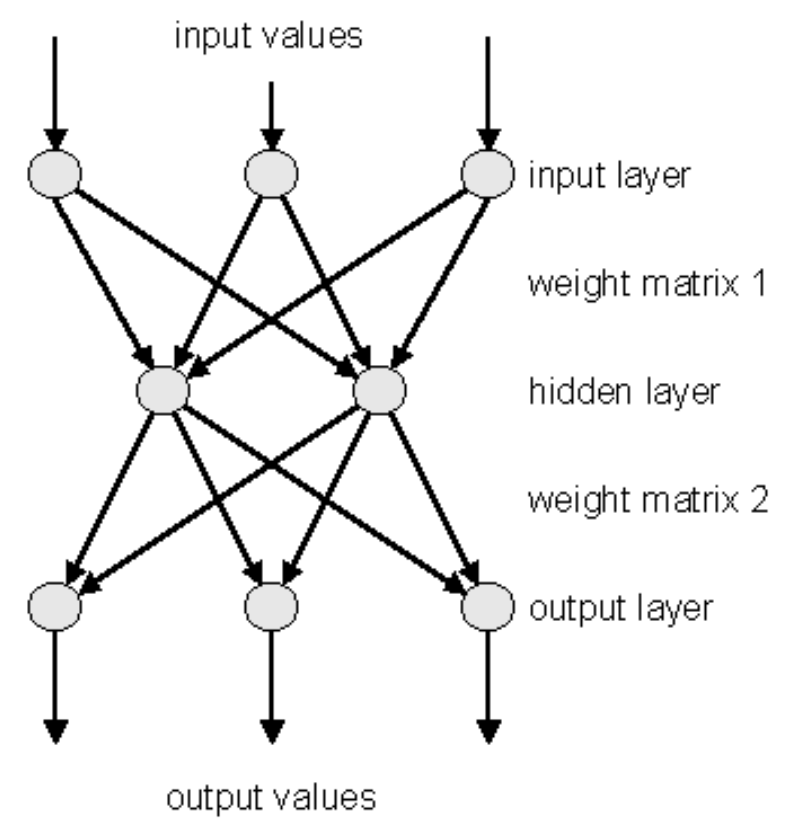

Figure 4.12: A typical architecture of an ANN. It is composed of an input layer, a hidden layer and an output layer.

The architecture of the final ANN is 11:6:6:1. It has one input layer, two hidden layers and one output layer. The input layer has 11 nodes which correspond to the 11 input variables. The output layer has one node. Each of the two hidden layers has 6 nodes. This neural network is often referred to as ANN11 in the $\nu_{e}$ analysis. We also tried other multivariate analysis algorithms including boosted decision trees and different implementations of neutral network in the framework of TMVA Toolkit [117] and they all gave very similar results in terms of signal efficiency and background rejection as obtained by the root class TMultiLayerPerceptron.

Fig.4.13 shows the ANN output for $\nu_{e}$ signal events and various background components. The signal distribution is scaled up by a factor of 10 for clarity. The ANN does a decent job in separating signal and background. However, there is a large overlapping between signal and background events. This is caused by the limited granularity of the MINOS detectors since they are not optimized for the $\nu_{e}$ search. 


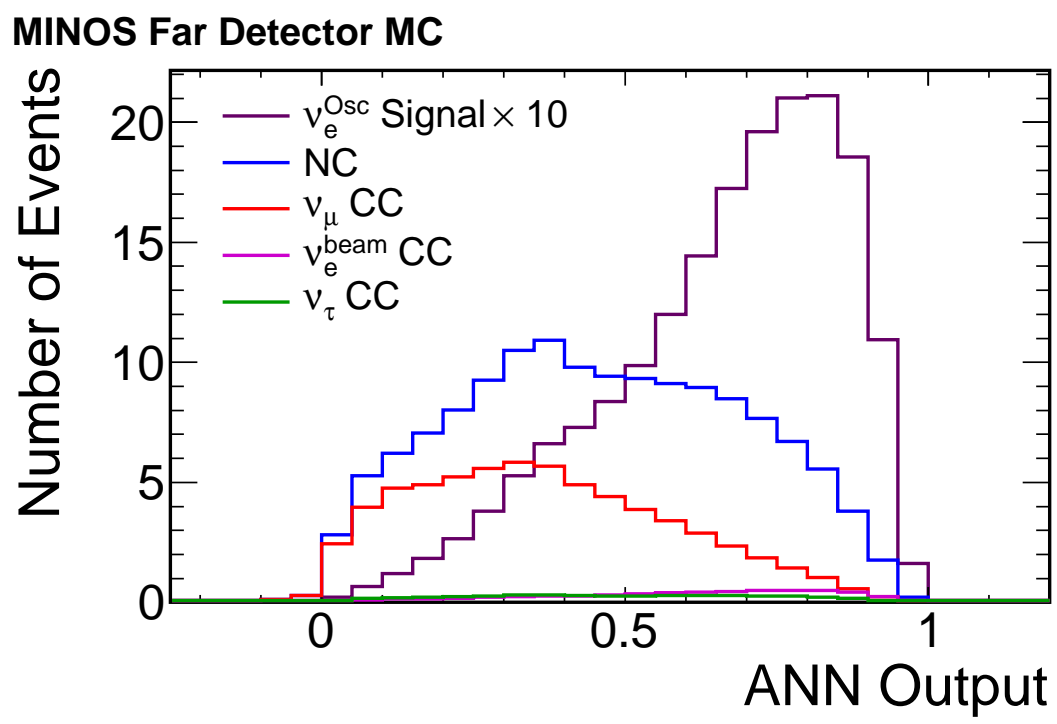

Figure 4.13: The ANN output for signal and different background components. The signal distribution is scaled by a factor of 10 for clarity. Pre-selections cuts are applied.

In order to optimize the cut on the ANN output, we choose this equation as the figure of merit (FOM):

$$
F O M \equiv N^{s i g} / \sqrt{\left(\sigma_{\text {stat }}^{b g}\right)^{2}+\left(\sigma_{\text {syst }}^{b g}\right)^{2}}=N^{\text {sig }} / \sqrt{N^{b g}+\left(10 \% \times N^{b g}\right)^{2}}
$$

where $N^{s i g}$ and $N^{b g}$ denote the numbers of signal events and background events, respectively. Here we assume a $10 \%$ systematic error on the number of predicted background events. This FOM was chosen because it represents the statistical significance of observed signal events with a given number of background events. It is directly related to the limit we can impose on the neutrino oscillation parameter $\theta_{13}$ if we do not observe any signal events in the Far Detector.

Fig.4.14 shows the FOM as a function of the cut on the ANN output. The signal is computed at $\mathrm{CHOOZ}$ limit. In order to get the maximum FOM, we shall place the 


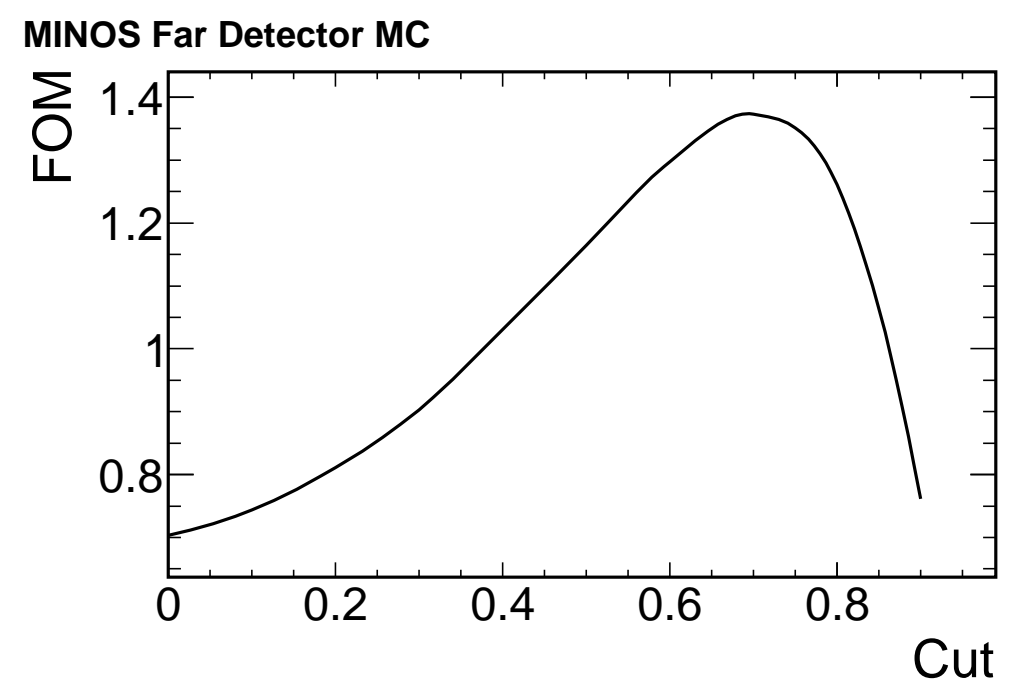

Figure 4.14: Figure of Merit (FOM) as a function of the cut on the ANN output. The signal is calculated at the CHOOZ limit.
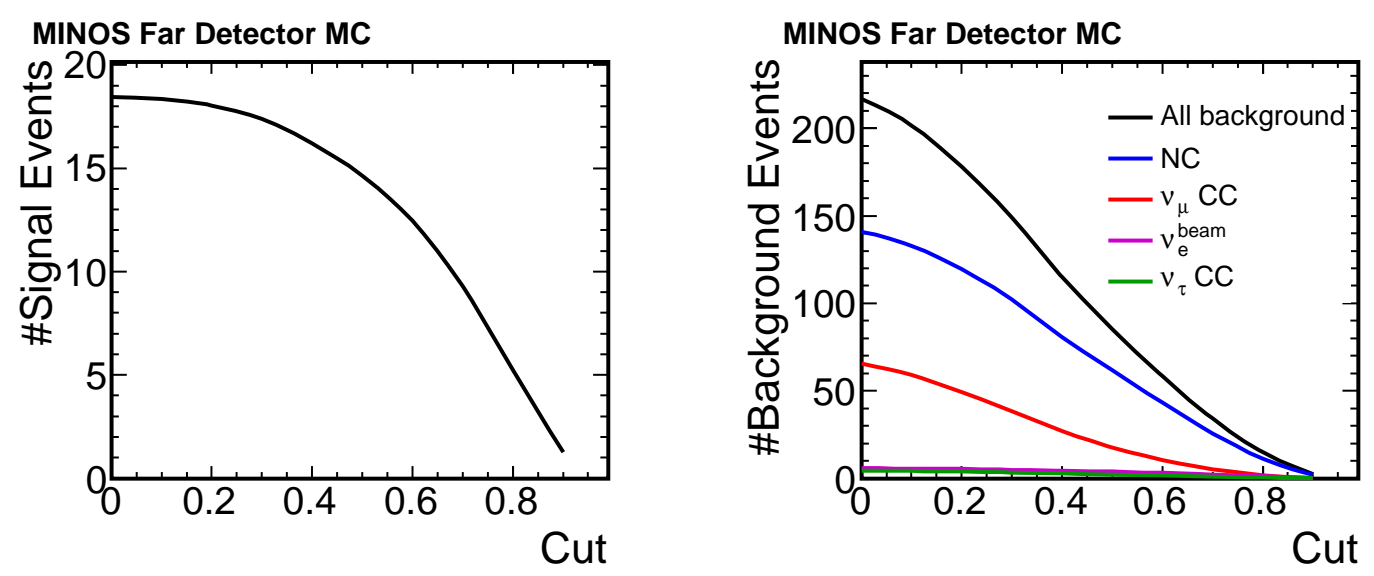

Figure 4.15: Number of accepted signal events and background events as a function of the cut on the ANN output. The signal is calculated at the CHOOZ limit. 
cut at 0.7 :

$$
\text { ANN output }>0.7
$$

The maximum FOM is 1.37. Fig.4.15 shows the number of accepted signal and background events as a function of the cut on the ANN output.

Table 4.1 summarizes the data reduction at different cut levels. This neural network based algorithm is able to accept roughly $39 \%$ of the signal events while rejecting $99 \%$ of the $\nu_{\mu} \mathrm{CC}$ background events and $93 \%$ of the NC background events. Fig.4.16 shows the background composition after applying all the $\nu_{e}$ selection cuts. The background is dominated by the $\mathrm{NC}$ interactions.

\begin{tabular}{c|ccccccc}
\hline & $\nu_{e}^{\text {osc }}$ & background & $\nu_{\mu} \mathrm{CC}$ & $\mathrm{NC}$ & $\nu_{e}^{\text {beam }} \mathrm{CC}$ & $\nu_{\tau} \mathrm{CC}$ & $\mathrm{FOM}$ \\
\hline Fid. Vol. Cuts & 23.6 & 1268.8 & 889.9 & 353.7 & 16.8 & 8.3 & 0.66 \\
\hline Pre-selection Cuts & 18.6 & 218.0 & 66.0 & 141.7 & 5.8 & 4.5 & 1.26 \\
\hline ANN11 PID $>0.7$ & 9.3 & 34.1 & 5.2 & 25.7 & 2.2 & 1.0 & 1.37 \\
\hline efficiency/rejection & $39.4 \%$ & $2.7 \%$ & $0.6 \%$ & $7.3 \%$ & $13.1 \%$ & $12.0 \%$ & \\
\hline
\end{tabular}

Table 4.1: Summary of Far Detector data reduction. The oscillation parameters are $\left|\Delta m_{31}^{2}\right|=2.4 \times 10^{-3} \mathrm{eV}^{2}, \sin ^{2} 2 \theta_{13}=0.15$ (CHOOZ limit), $\sin ^{2} 2 \theta_{23}=1$. We assume $\mathrm{POT}=3.25 \times 10^{20}$ (2-year exposure). FOM is defined as $N^{s i g} / \sqrt{N^{b g}+\left(10 \% \times N^{b g}\right)^{2}}$.

The following plots show the performance of the ANN. Fig.4.17 shows the reconstructed energy distributions of events after applying all the $\nu_{e}$ selection cuts. Fig.4.18 and Fig.4.19 show the energy spectra of background events and signal events at various $\nu_{e}$ selection cut levels. Fig.4.20 shows the $\nu_{e}$ selection efficiency and purity as a function of energy. The $\nu_{e}$ selection efficiency decreases at high energies as contrary to what one would expect since it is usually easy to identify high energy electrons. The reason may be that we weigh the signal events by the oscillation probabilities in the training sample thus high energy $\nu_{e}$ events are highly suppressed. The neural network therefore preferably selects low energy $\nu_{e}$ events. Note that we also apply a 


\section{Background composition}

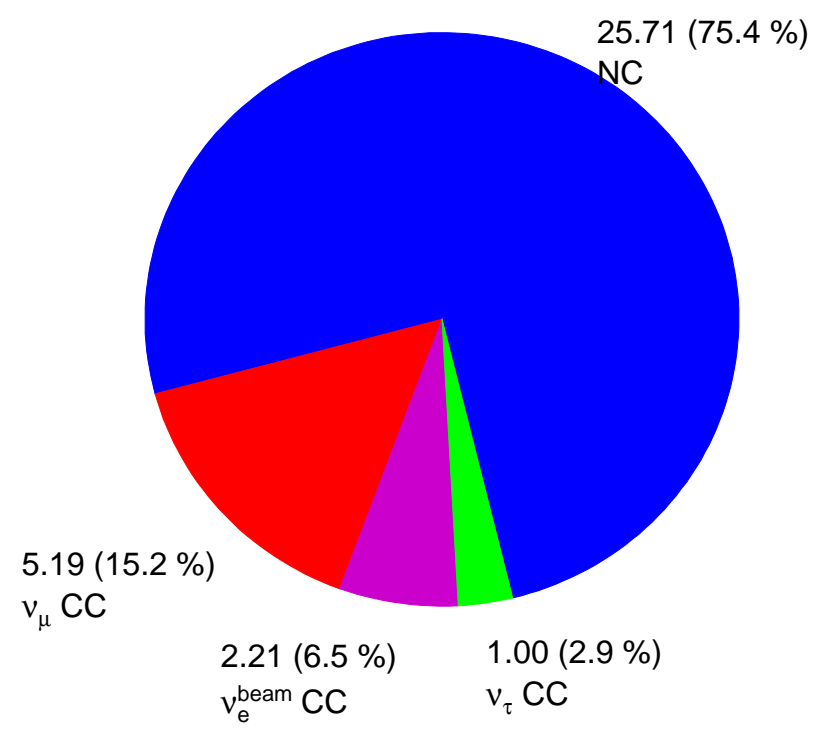

Figure 4.16: Far Detector background composition after applying all the $\nu_{e}$ selection cuts.

high energy pre-selection cut $E<8 \mathrm{GeV}$ to eliminate high energy beam $\nu_{e}$ background from kaon decays.Fig.4.21 shows the breakdown of Far Detector accepted signal and background events as number of $\pi^{0}$ 's in the final state. The signal is dominated by the $0 \pi^{0}$ events while the background is dominated by the $\operatorname{single}-\pi^{0}$ and multi- $\pi^{0}$ events. Fig.4.22 shows the breakdown of Far Detector accepted signal and background events as the interaction type. The signal has almost equal contributions from QE, RES and DIS interactions while the background is dominated by the DIS interactions.

\subsection{Near Detector Data Monte Carlo Comparisons}

We have described the ANN we constructed based on the Far Detector Monte Carlo. It is very important to understand the Near Detector data in terms of the detector 


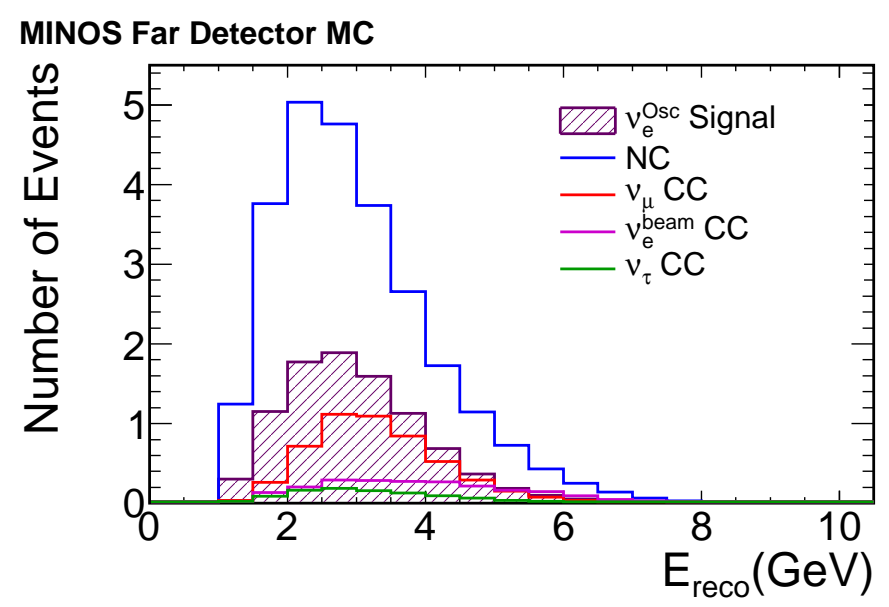

Figure 4.17: Reconstructed energy distributions of events that are accepted by the ANN as $\nu_{e}$-like events.
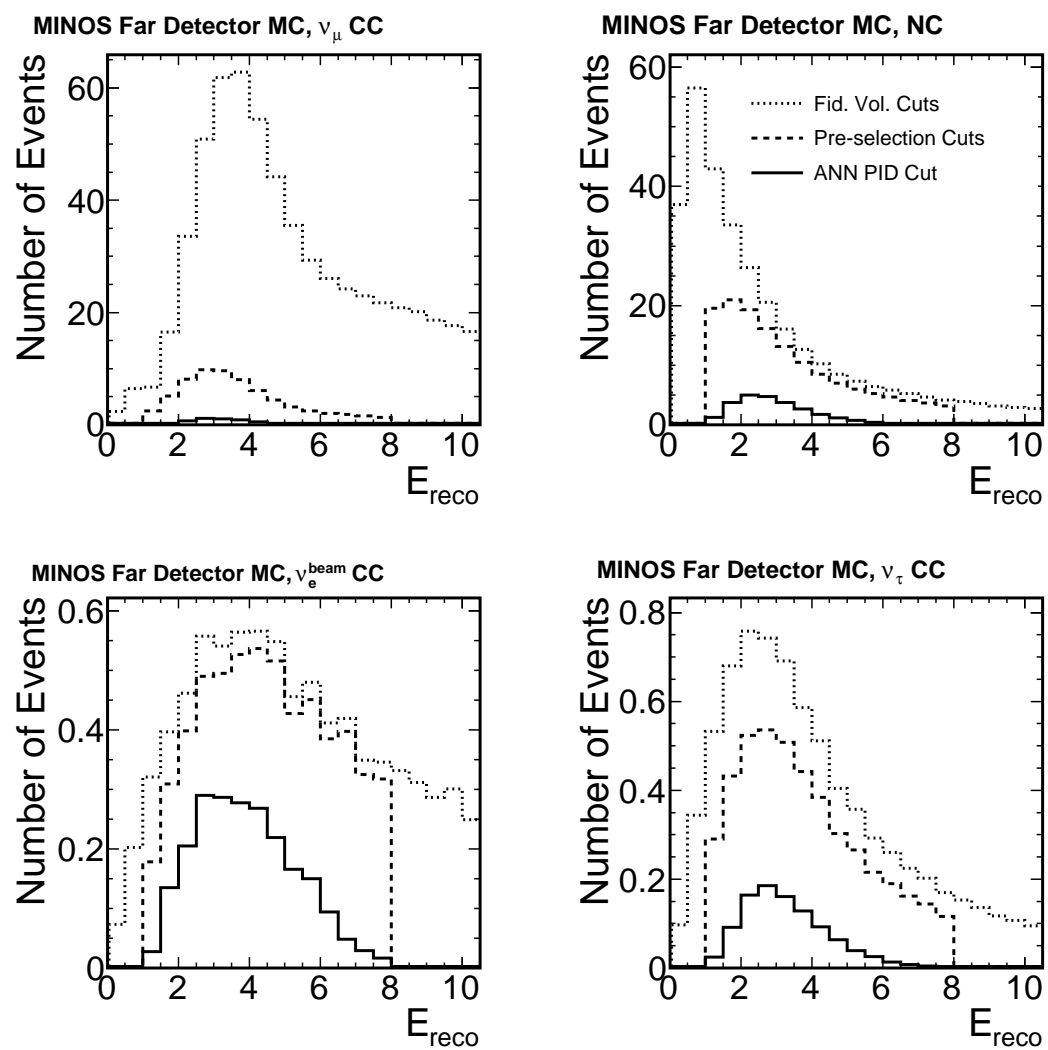

Figure 4.18: Energy spectra of background events at various $\nu_{e}$ selection cut levels. 


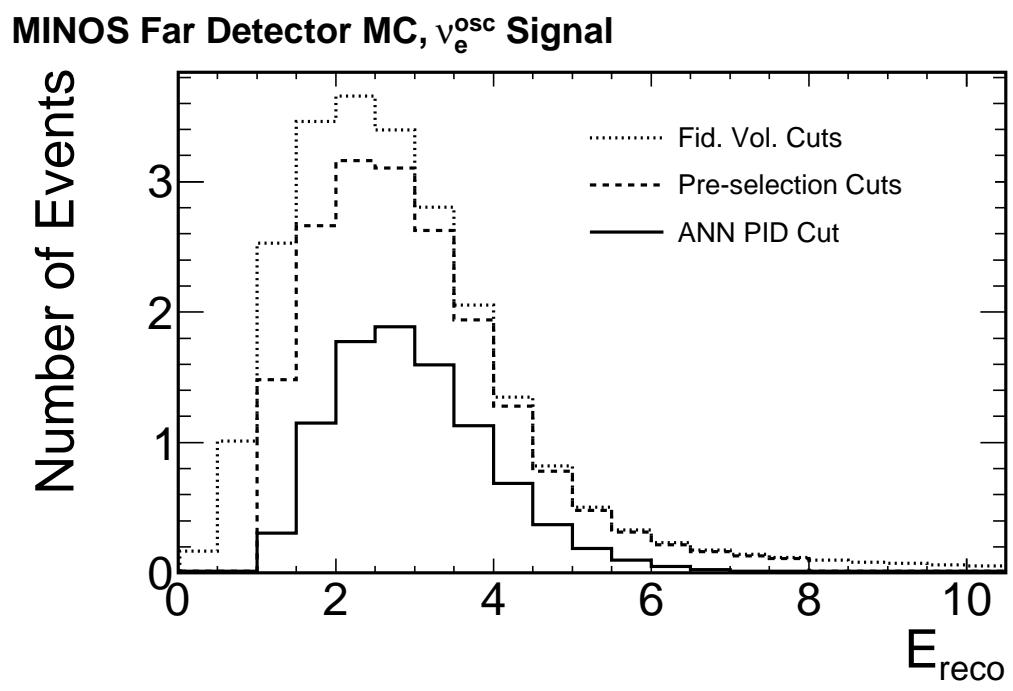

Figure 4.19: $\nu_{e}$ Energy spectra of signal events at various $\nu_{e}$ selection cut levels.

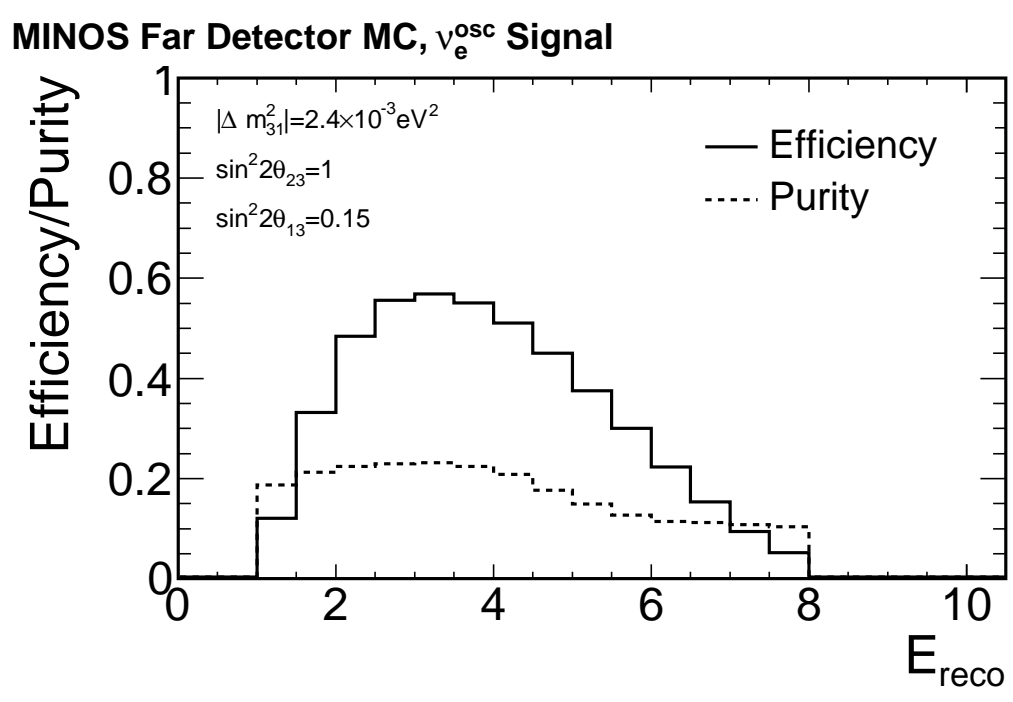

Figure 4.20: $\nu_{e}$ selection efficiency and purity. 
$v_{e}^{\text {osc }}$ Signal

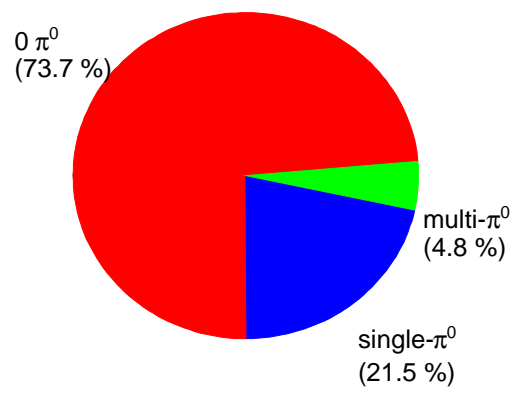

Background

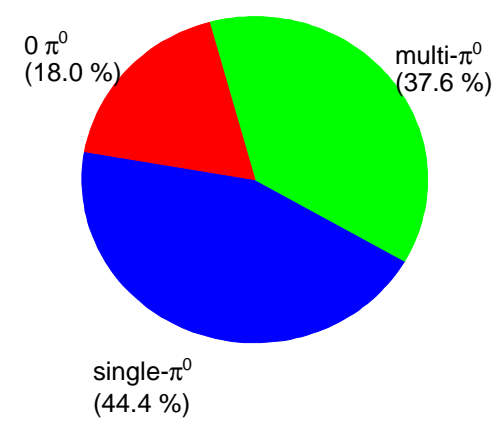

Figure 4.21: Breakdown of Far Detector accepted signal and background events as number of $\pi^{0}$ 's in the final state.

$v_{\mathrm{e}}^{\text {osc Signal }}$

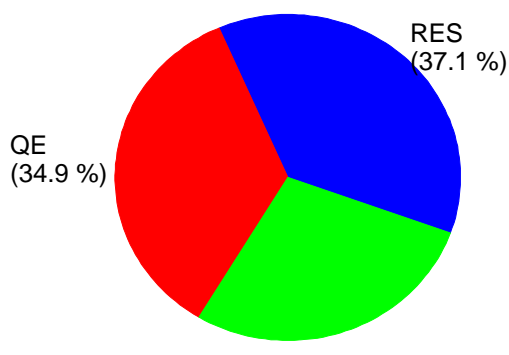

DIS
$(28.0 \%)$
Background

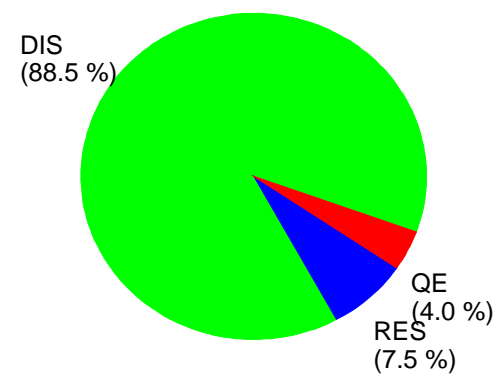

Figure 4.22: Breakdown of Far Detector accepted signal and background events as the interaction type. 
performance, and the event reconstruction. The purpose of the MINOS Near Detector for the $\nu_{e}$ analysis is to measure and study the background rate. Near Detector measurements will verify and check the accuracy of the neutrino beam simulation, cross section modeling and detector response simulation. This task is of great importance for the Far Detector background prediction. In this section, we will show the Near Detector data and MC comparisons for some of the $\nu_{e}$ pre-selection variables, the ANN11 PID, and the energy spectra of selected $\nu_{e}$ candidates.

We sampled one sixth of the ND data taken between May 2005 and February 2007 as our data sample. The total number of protons on target (POTs) for the selected ND data sample is $4.55 \times 10^{19}$. The total ND MC sample used corresponds to $1.77 \times 10^{20}$ POT. We apply cuts to remove events when the beam parameters failed the "good beam" conditions and when the detector had some malfunction. The Near Detector fiducial volume cuts we use in the $\nu_{e}$ analysis are:

$$
\sqrt{(x-1.4885)^{2}+(y-0.1397)^{2}} \leq 0.8,1 \leq z \leq 5
$$

where $(\mathrm{x}, \mathrm{y}, \mathrm{z})$ are the coordinates of the reconstructed event vertex, and $\mathrm{z}$ axis is along the beam direction. Coordinates $(1.4885 \mathrm{~m}, 0.1397 \mathrm{~m})$ represent the position of the beam center on the detector front face. In the comparison Figures below, both the data and $\mathrm{MC}$ distributions are normalized to $1 \times 10^{19}$ POTs.

Fig.4.23 shows the track length in number of planes that the track traverses for events in the fiducial volume. Track-like planes refer to the "clean" hit planes in the track, a clean plane is one in which the plane is free from the presence of non-track associated hits. The MC simulation agrees reasonably well with the data.

Fig.4.24 shows the reconstructed energy distributions for events passing the fiducial volume cuts and the track length cuts. In the energy range we are interested in $(1 \mathrm{GeV}$ to $8 \mathrm{GeV})$, the $\mathrm{MC}$ agrees with data within $10 \%$. 

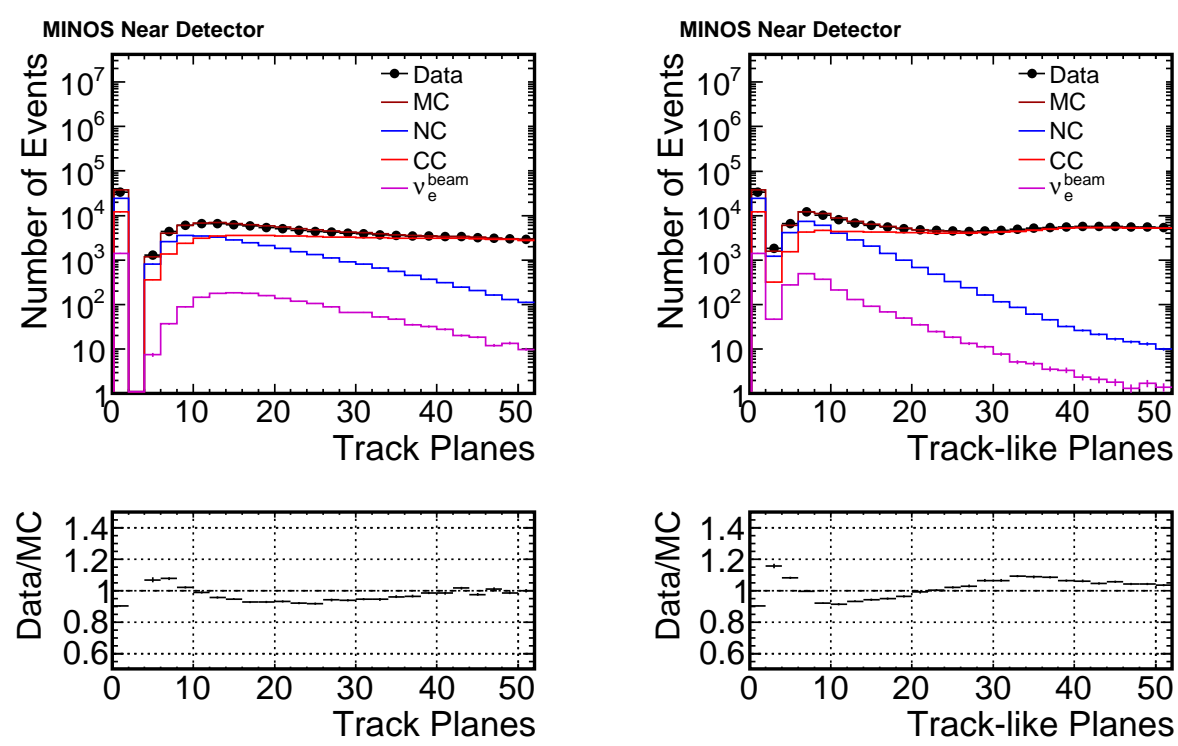

Figure 4.23: ND Track planes and track-like planes distributions after fiducial volume cuts.
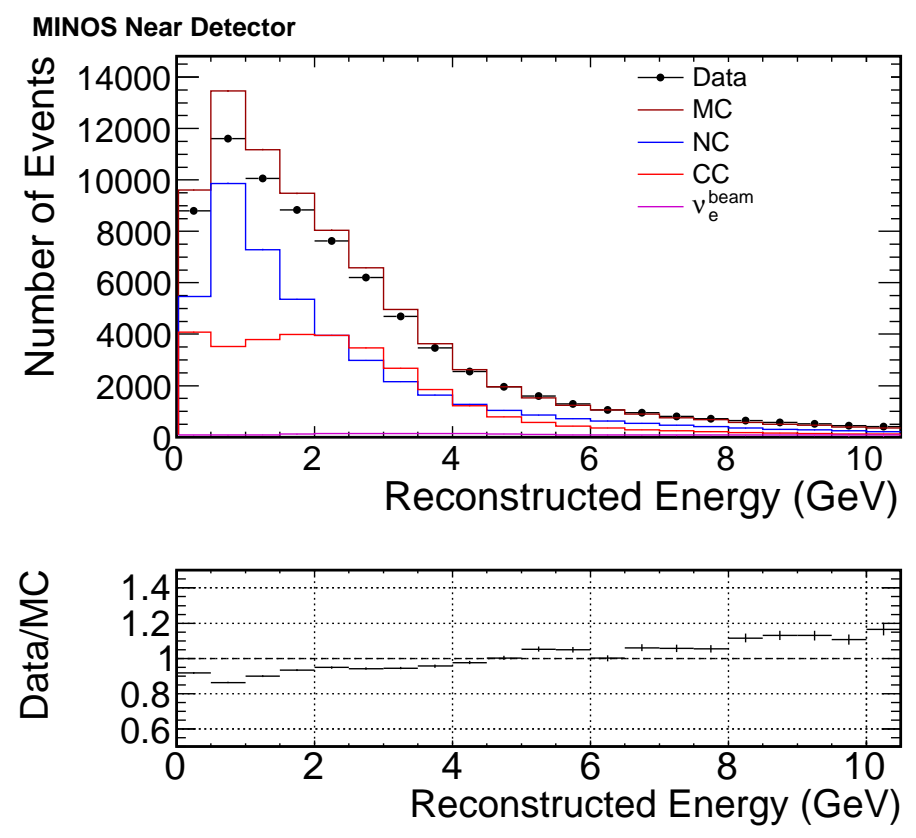

Figure 4.24: ND Reconstructed energy distributions after fiducial volume cuts and track length cuts. 
Fig.4.25 shows the distributions of the 11 variables which are inputs to the ANN. In computing these variables, we use only hits whose pulse-heights are greater than 2 PE to eliminate the PMT crosstalk hits. Data and MC agree within $20 \%$ for most of the distributions.

Fig.4.26 shows the ANN output for the ND data and MC events that pass the $\nu_{e}$ pre-selection cuts. The breakdown of MC distribution is also shown. Fig.4.27 shows the reconstructed energy spectra of the data and MC events accepted by the ANN PID as $\nu_{e}$ candidates (ANN11>0.7).

Apparently the MC simulation does not represent the data very well especially in the region we are interested in (highly $\nu_{e}$-like events). The $\nu_{e}$ selection algorithm selects only a very small fraction of the total events. It turns out that the modeling of those particular events (highly $\nu_{e}$-like, usually with a big $\pi^{0}$ contribution) is very sensitive to many aspects of the MC simulation, which include the detailed modeling of the hadrons resulting from neutrino interactions, in particular the $\pi^{0}$ component, and the hadron intranuclear rescattering. Studies have shown that tweaking those aspects of the MC simulation can vary the MC background rate by as much as $30 \%$. However, the method we adopted for the Far Detector background rate prediction is rather independent of the uncertainties in the physics simulation. We measure the background rate at the Near Detector, which we extrapolate to the Far Detector using the $\mathrm{MC}$ information to get the Far Detector background prediction:

$$
N_{\text {Far }}^{\text {Predicted }}=N_{\text {Near }}^{\text {Data }} \times\left(\frac{N_{\text {Far }}}{N_{\text {Near }}}\right)^{M C}
$$

The measurements we perform at the Near Detector are only statistically limited and the statistical error is negligible since we have almost infinite data at the ND. A lot of uncertainties especially the ones on the physics simulation cancel in the MC far over near ratio. This is the advantage of having two detectors in a neutrino oscillation 

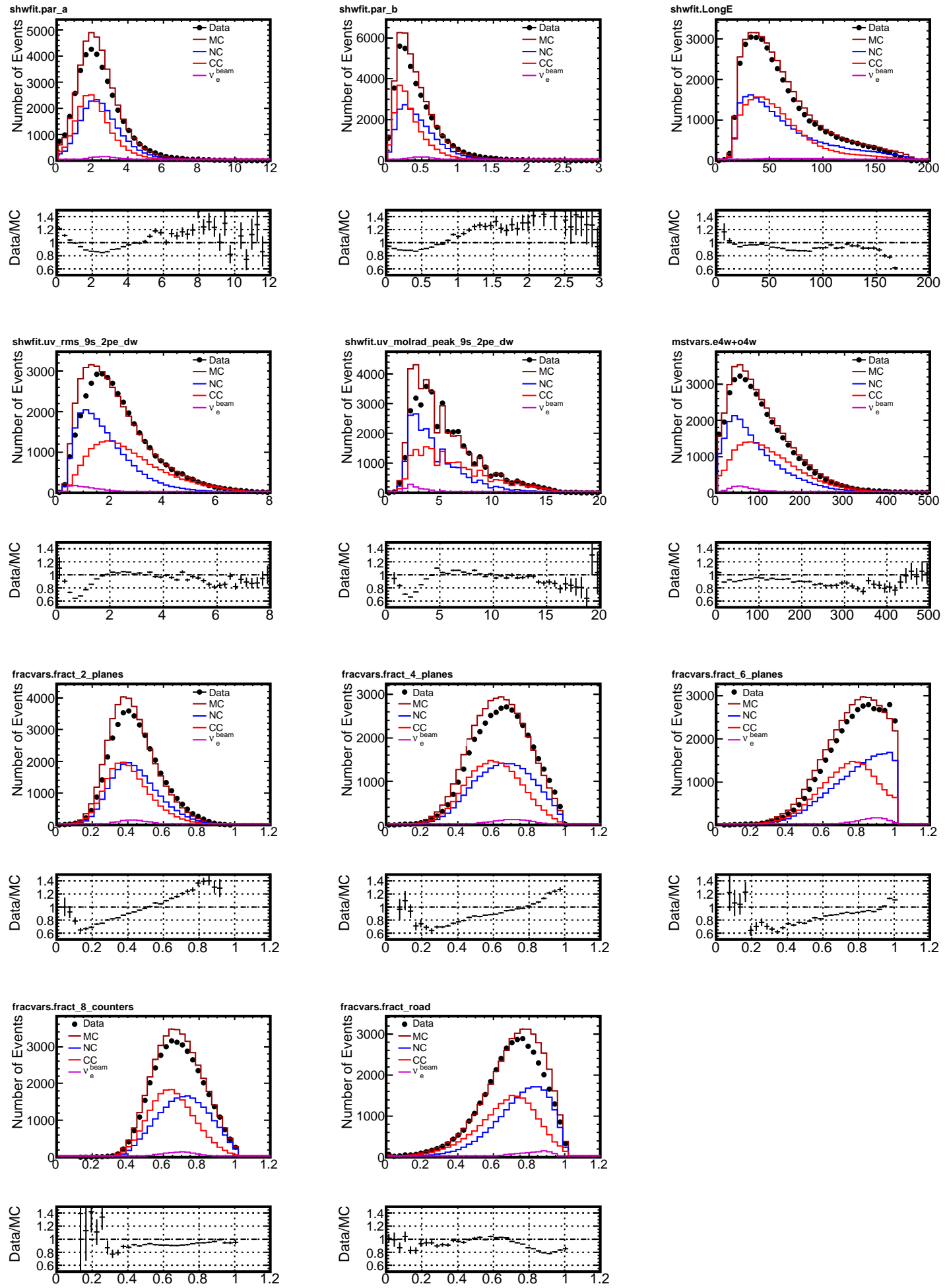

Figure 4.25: Distributions of the 11 variables used in the artificial neural network. Both data and MC are normalized to $1 \times 10^{19}$ POTs and only events passing the pre-selection cuts are used in making these plots. 

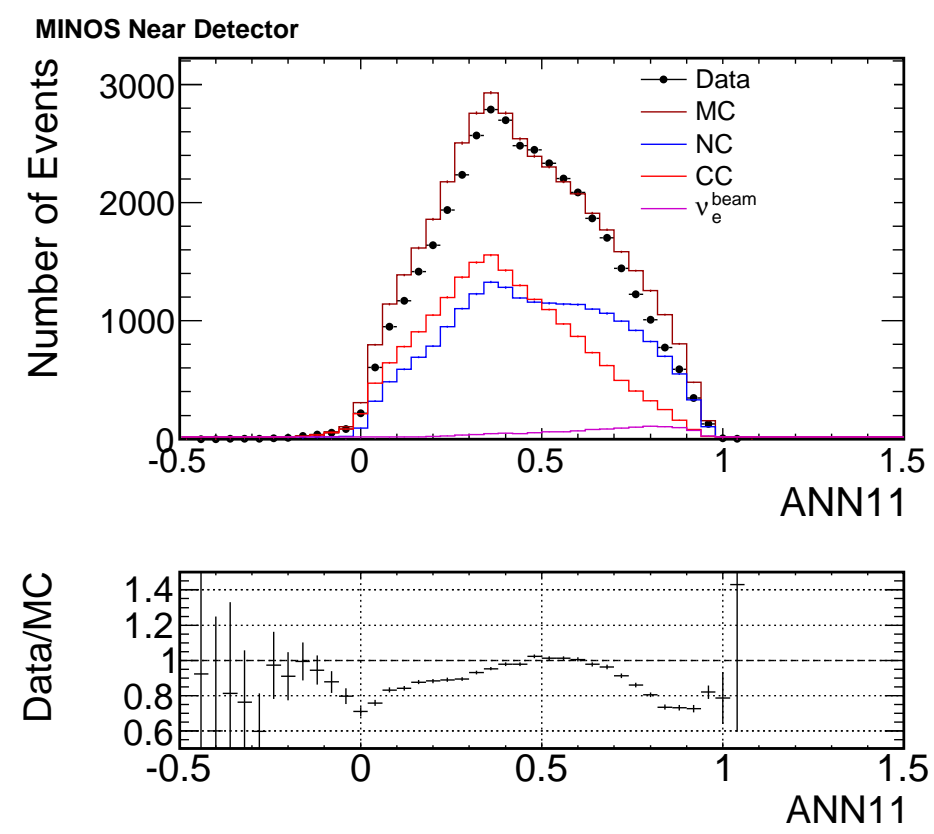

Figure 4.26: The ANN output for signal and different background components. Preselections cuts are applied.
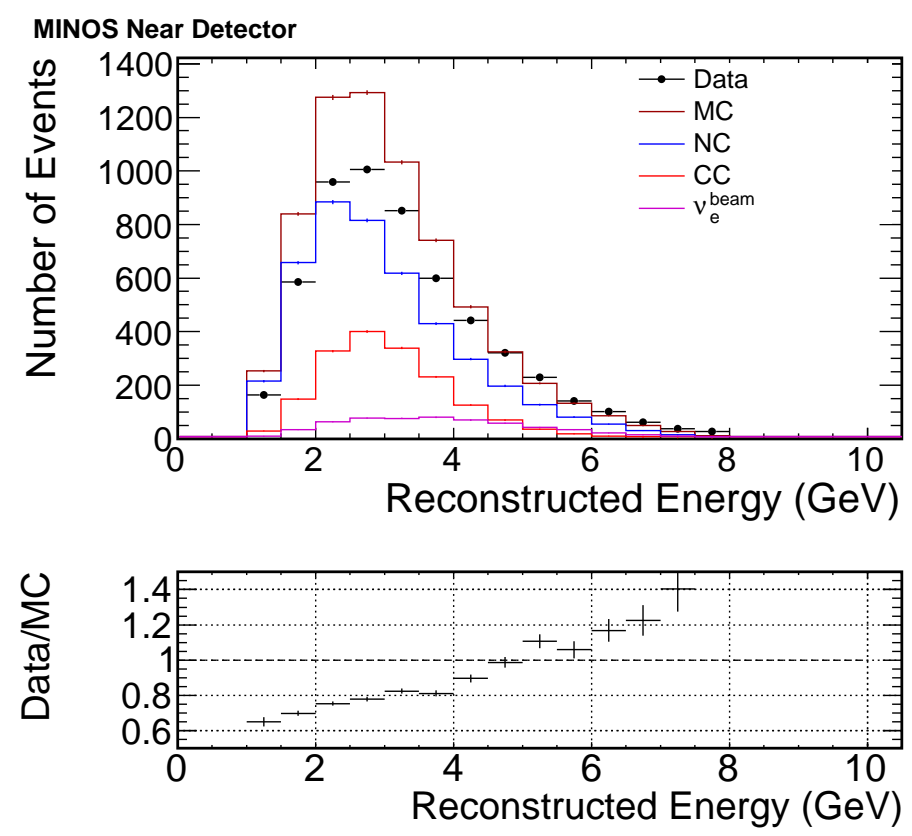

Figure 4.27: Reconstructed energy distributions of events that are accepted by the ANN as $\nu_{e}$-like events. 
experiment. We believe the Far Detector background prediction is quite robust even though we may have a large systematic error on the physics simulation. Now we demonstrate this with an example. In 2007, the MINOS physics simulation group discovered that the treatment of hadron intranuclear rescattering in the simulation program intranuke was not consistent with the external measurements made by the bubble chamber experiments. The hadron absorption probability was underestimated by roughly $10 \%$. After the intranuke model was corrected, a new set of MC was generated. The comparison of the $\mathrm{MC}$ with the improved intranuke model to the $\mathrm{MC}$ with the wrong (default) intranuke model was depicted in Fig.4.28 for the ND (a) and FD (b) MC. Changing the intranuke model changes the energy spectra of events that pass the $\nu_{e}$ PID selection by roughly $10 \%$. However, the changes go in the same direction between the ND and the FD, thus one would expect the effect on the far/near ratio is minimal. Fig.4.29 shows the Far/Near ratios as a function of reconstructed energy for $\mathrm{NC}$ events and $\nu_{\mu} \mathrm{CC}$ events that are accepted as $\nu_{e}$ candidates. The ratios are indeed very consistent between the two MC samples. The $\mathrm{NC}$ ratios are relatively flat because the neutrino oscillations do not change the $\mathrm{NC}$ event rate at the FD. The CC ratios are lower at low energies due to the $\nu_{\mu} \rightarrow \nu_{\tau}\left(\nu_{e}\right)$ oscillations while the ratios flatten as energy increases since the oscillations are suppressed at higher energies. We do not show the far/near ratio for the beam $\nu_{e}$ events here since their contribution to the FD background rate is small and thus is taken from $\mathrm{MC}$, i.e. not from extrapolation from the ND. The number of $\nu_{\tau} \mathrm{CC}$ background events at FD is also estimated from MC since they do not exist at ND.

Table 4.2 summarizes the numbers of accepted events at both detectors for the two $\mathrm{MC}$ sets. Changing the intranuke model can change the numbers of $\mathrm{NC}$ and $\nu_{\mu} \mathrm{CC}$ background events by $10 \%$ to $15 \%$ at each individual detector, but the far/near ratios remain unchanged within statistics. This result indicates that the MC far/near ratio is quite robust and largely independent of the uncertainties in the physics simulation. 

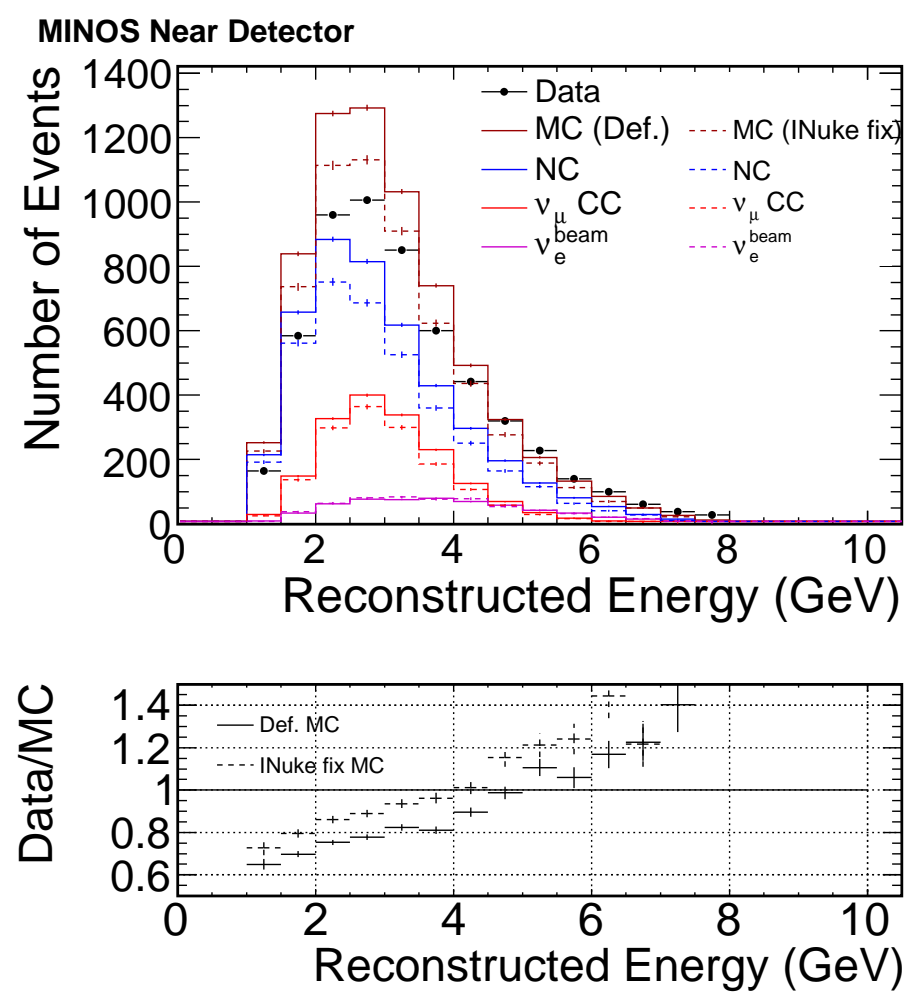

(a) Near Detector

\section{MINOS Far Detector}

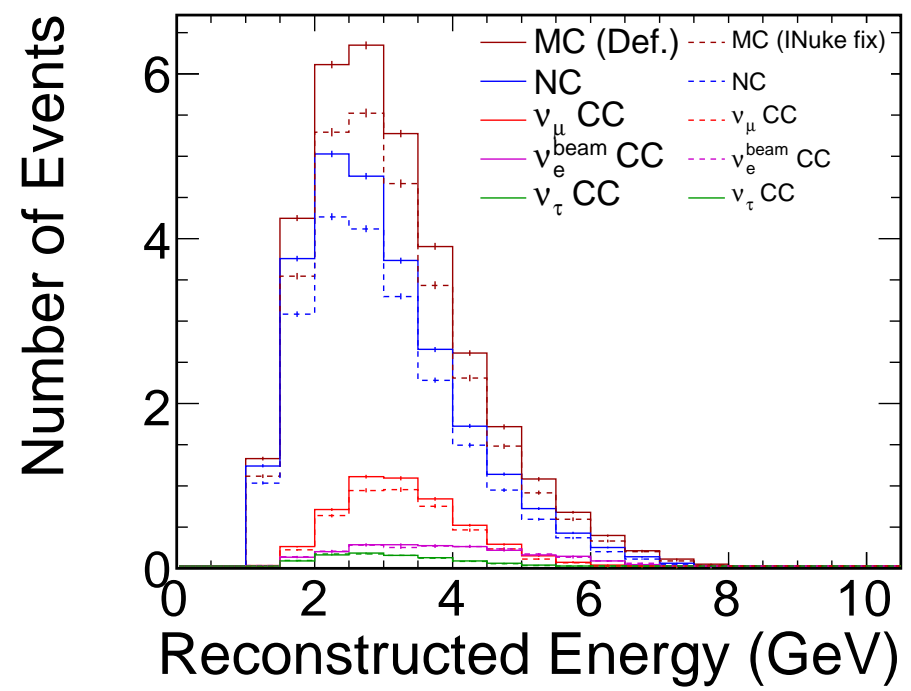

(b) Far Detector

Figure 4.28: Effects of changing hadron intranuclear rescattering on the background rates (a) ND $\left(1 \times 10^{19} \mathrm{POTs}\right)$ and (b) FD $\left(3.25 \times 10^{20} \mathrm{POTs}\right)$. The solid lines represent the MC with the default intranuke model while the dashed lines represent the $\mathrm{MC}$ with the improved intranuke model. The nominal oscillation probabilities are applied to the FD spectra. 

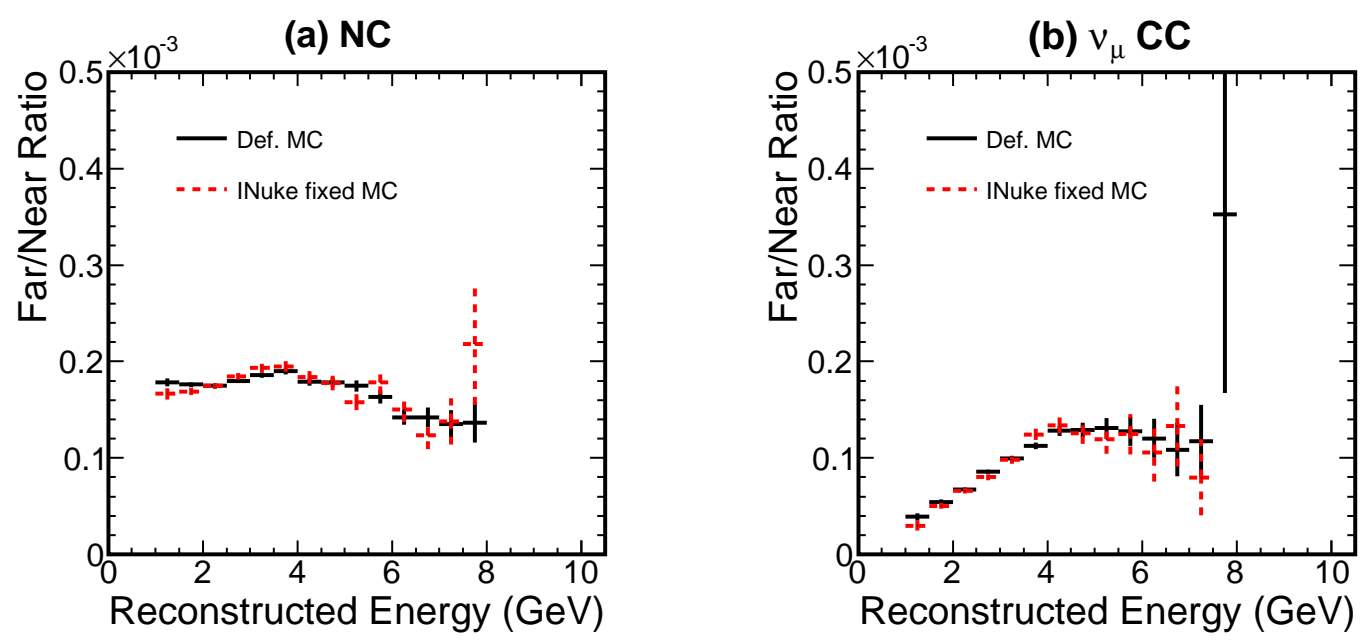

Figure 4.29: Far/Near ratios as a function of reconstructed energy for NC events and $\nu_{\mu} \mathrm{CC}$ events that are accepted as $\nu_{e}$ candidates. The nominal oscillation probabilities are applied to the FD spectra. ND MC and FD MC are normalized to the same exposure.

\begin{tabular}{|c|c|c|c|c|c|c|}
\hline & & $\nu_{e}^{o s c}$ & $\mathrm{NC}$ & $\nu_{\mu} \mathrm{CC}$ & $\nu_{e}^{\text {beam }} \mathrm{CC}$ & $\nu_{\tau} \mathrm{CC}$ \\
\hline \multirow{3}{*}{ FD } & MC(Def.) & $9.29 \pm 0.02$ & $25.71 \pm 0.09$ & $5.19 \pm 0.05$ & $2.21 \pm 0.04$ & $1.00 \pm 0.01$ \\
\hline & MC(INu.) & $9.17 \pm 0.02$ & $21.90 \pm 0.10$ & $4.49 \pm 0.06$ & $2.20 \pm 0.05$ & $0.98 \pm 0.01$ \\
\hline & $\mathrm{INu} /$ Def-1 & $-1.3 \% \pm 0.3 \%$ & $-14.8 \% \pm 0.5 \%$ & $-13.5 \% \pm 1.5 \%$ & $-0.5 \% \pm 3.1 \%$ & $-1.8 \% \pm 1.7$ \\
\hline \multirow{3}{*}{ ND } & MC(Def.) & & $4429 \pm 17$ & $1742 \pm 10$ & $593 \pm 6$ & \\
\hline & MC(INu.) & & $3758 \pm 27$ & $1539 \pm 16$ & $611 \pm 10$ & \\
\hline & $\mathrm{INu} /$ Def-1 & & $-15.1 \% \pm 0.7 \%$ & $-11.7 \% \pm 1.1 \%$ & $3.0 \% \pm 2.0 \%$ & \\
\hline \multicolumn{2}{|c|}{$(\mathrm{F} / \mathrm{N})_{\mathrm{I}} /(\mathrm{F} / \mathrm{N})_{\mathrm{D}^{-1}}$} & & $0.4 \% \pm 1.0 \%$ & $-2.1 \% \pm 2.0 \%$ & $-3.4 \% \pm 3.6 \%$ & \\
\hline
\end{tabular}

Table 4.2: Numbers of accepted events at both detectors for MC with default intranuke model (Def.) and $\mathrm{MC}$ with the corrected intranuke model (INu.). The oscillation parameters are $\left|\Delta m_{31}^{2}\right|=2.4 \times 10^{-3} \mathrm{eV}^{2}, \sin ^{2} 2 \theta_{13}=0.15, \sin ^{2} 2 \theta_{23}=1$. The numbers are normalized to FD POT $=3.25 \times 10^{20}$ (2-year exposure) and ND $\mathrm{POT}=1 \times 10^{19}$. The errors are only statistical errors. 
The uncertainties in the hadronization and intranuclear rescattering processes do not really affect genuine $\nu_{e}$ events since their most relevant feature is electromagnetic shower development which is well modeled according to CalDet data and MC comparisons.

\subsection{Summary}

In this chapter, we have described the strategy of identifying $\nu_{e} \mathrm{CC}$ events. We first showed the characteristics of EM shower and hadronic show in MINOS detectors using CalDet data. We then described the separation method, focusing on a neural network based $\nu_{e}$ selection algorithm. The ANN technique provides a useful method of selecting $\nu_{e} \mathrm{CC}$ interactions for the $\nu_{e}$ appearance analysis. It accepts $40 \%$ of the $\nu_{e}$ signal events while rejecting $99 \%$ of the $\nu_{\mu} \mathrm{CC}$ and $93 \%$ of the $\mathrm{NC}$ background events. We also described how we predict FD background using ND data and MC Far/Near ratios. Finally we showed that our method of FD background prediction is robust to physics uncertainties. 


\section{Chapter 5}

\section{MINOS Hadronization Model}

\section{$5.1 \quad$ Introduction}

Neutrino-induced hadronic shower modeling is an important aspect of neutrino interactions in the few-GeV range. The hadronization model (often referred to as fragmentation model) refers to the part of simulation that determines the final state particles and 4-momenta given the nature of a neutrino-nucleon interaction $(\mathrm{CC} / \mathrm{NC}, \nu / \bar{\nu}$, target neutron/proton) and the event kinematics $\left(W^{2}, Q^{2}, x, y\right.$ etc). The hadronization model is very important for the MINOS experiment, especially for the shower modeling. For the $\nu_{\mu} \rightarrow \nu_{e}$ appearance analysis, as we have shown in the previous chapter, the background is dominated by the $\pi^{0}$ 's generated via $\mathrm{NC}$ interaction. Thus the evaluation of background coming from $\mathrm{NC}$ events is quite sensitive to the details of the NC shower simulation and specifically the $\pi^{0}$ shower content.

The original hadronization model that MINOS used was a KNO-based [120] empirical model implemented in the NEUGEN Monte Carlo generator [101]. The old KNO model was tuned to reproduce the experimentally measured total hadronic multiplicities but had some drawbacks, which originated from the treatment of secondary hadrons in the hadronic center-of-mass system (c.m.s.). The old KNO model assumed 
that those hadrons are isotropically distributed in the hadronic c.m.s. But in reality, the baryon of the shower particle content tends to propagate in the backward direction $\left(x_{F}<0\right)$ while the mesons propagate in the forward direction - current direction $\left(x_{F}>0\right)^{1}$. The consequence is that the mesons acquire more energy than the baryon does when boosted to the LAB frame.

In order to improve the MINOS MC simulation, we have developed a new hadronization model, namely the Andreopoulos-Gallagher-Kehayias-Yang (AGKY) model to replace the old one. We use the PYTHIA/JETSET [105] model to simulate the hadronic showers at high hadronic invariant masses. We also developed a phenomenological description of the low invariant mass hadronization since the validity of the PYTHIA/JETSET model, for neutrino-induced showers, is known to deteriorate as one approaches the pion production threshold. The phenomenological model we developed is also a KNO-based model but with a lot of improvements over the old model. This chapter focuses on the detailed description of the AGKY hadronization model and the tuning and validation of this model using bubble chamber experimental data.

\subsection{The AGKY Model}

\subsubsection{Overview}

The AGKY model, which is now the default hadronization model in the neutrino Monte Carlo generators NEUGEN [101] and GENIE-2.0.0 [118], includes a KNObased effective description of the low invariant mass neutrino-induced hadronization while at higher masses it gradually switches over to the PYTHIA/JETSET model. The transition from the KNO model to the PYTHIA/JETSET model takes place gradually, at an intermediate invariant mass region, ensuring the continuity of all

\footnotetext{
${ }^{1} x_{F}=P_{L}^{*} / P_{L \max }^{*}=$ Feynman- $x \simeq P_{L}^{*} /(W / 2)$, where $P_{L}^{*}$ is the longitudinal momentum of the particle in the hadronic c.m.s and $P_{L \max }^{*}$ is its maximum kinematic value.
} 
simulated observables as a function of the invariant mass. This is accomplished by using a transition window $\left[W_{m i n}^{t r}, W_{m a x}^{t r}\right]$ over which we linearly increase the fraction of neutrino events for which the hadronization is performed by the PYTHIA/JETSET model from $0 \%$ at $W_{\min }^{t r}$ to $100 \%$ at $W_{\max }^{t r}$. The default values used in the AGKY model are:

$$
W_{\min }^{t r}=2.3 \mathrm{GeV} / c^{2}, W_{\max }^{t r}=3.0 \mathrm{GeV} / c^{2}
$$

The typical kinematic regions over which the different hadronization models apply are shown graphically in Fig.5.1 superimposed on top of the expected invariant mass distribution of the hadronic system in the neutrino interactions in the MINOS Near Detector using the NuMI muon neutrino beam [57]. The shaded area shows the resonance contribution for which the Rein-Sehal model [102] is used. This model, including the resonances and a non-resonant background, describes the $N \pi$ mass distributions, the cross section as functions of neutrino energy, and the steeply falling $Q^{2}$ distributions at low $W<1.4 \mathrm{GeV}$ reasonably well.

The main thrust of this work was to develop the model at the low invariant mass regime which is the region of the MINOS neutrino oscillation signal. The description of AGKY's KNO model, used at low invariant masses, can be split into two independent parts:

- Generation of the hadron shower particle content

- Generation of hadron 4-momenta

These two will be described in detail in the following sections. 


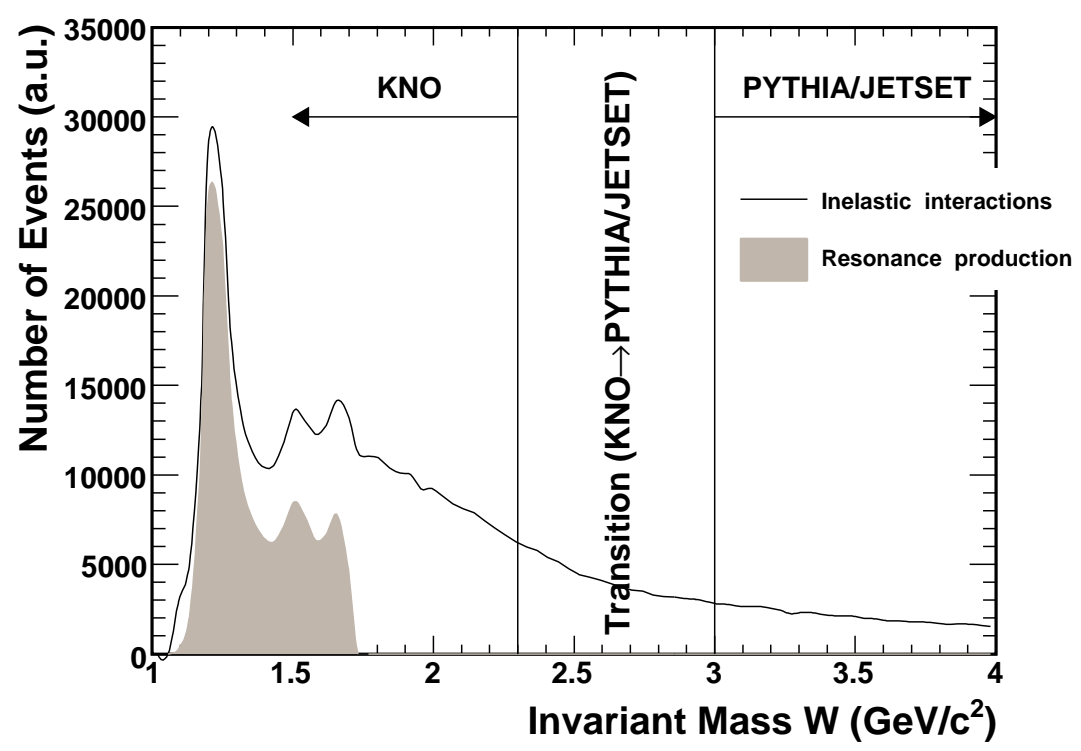

Figure 5.1: Simulated invariant mass distribution of inelastic events in the MINOS Near Detector using the NuMI muon neutrino beam. The shaded area shows the resonance contribution for which a different hadronization model (Rein-Sehgal) is employed. 
The neutrino interactions are often described by the following kinematic variables:

$$
\begin{aligned}
Q^{2} & =2 E_{\nu}\left(E_{\mu}-p_{\mu}^{L}\right)-m^{2} \\
\nu & =E_{\nu}-E_{\mu} \\
W^{2} & =M^{2}+2 M \nu-Q^{2} \\
x & =Q^{2} / 2 M \nu \\
y & =\nu / E_{\nu}
\end{aligned}
$$

where $Q^{2}$ is the invariant 4-momentum transfer squared, $\nu$ is the neutrino energy transfer, $W$ is the effective mass of all secondary hadrons (invariant hadronic mass), $x$ is the Bjorken scale variable, $y$ is the relative energy transfer, $E_{\nu}$ is the incident neutrino energy, $E_{\mu}$ and $p_{\mu}^{L}$ are the energy and longitudinal momentum of the muon, $M$ is the nucleon mass and $m$ is the muon mass.

For each hadron in the hadronic system, we define the variables $z=E_{h} / \nu, x_{F}=$ $2 p_{L}^{*} / W$ and $p_{t}$ where $E_{h}$ is the energy in the laboratory frame, $p_{L}^{*}$ is the longitudinal momentum in the hadronic c.m.s., and $p_{T}$ is the transverse momentum with respect to the momentum transfer direction.

\subsubsection{Low- $W$ model: Particle content}

At low invariant masses the AGKY model generates hadronic systems that typically consist of exactly one baryon ( $p$ or $n$ ) and any number of $\pi^{+}, \pi^{-}, \pi^{0}, K^{+}, K^{-}, K^{0}, \overline{K^{0}}$ mesons that are kinematically possible and in accordance with charge conservation.

For a fixed hadronic invariant mass and initial state (neutrino and struck nucleon), the algorithm for generating the hadron shower particles generally proceeds as follows: 
- Compute the average charged hadron multiplicity using the empirical expression:

$$
\left\langle n_{c h}\right\rangle=a+b \ln W^{2}
$$

The coefficients $a, b$, which depend on the initial state, have been determined by bubble chamber experiments.

- Compute the average hadron multiplicity as $\left\langle n_{t o t}\right\rangle=1.5\left\langle n_{c h}\right\rangle[119]$.

- Generate the actual hadron multiplicity taking into account that the multiplicity dispersion is described by the KNO scaling law [120]:

$$
\langle n\rangle \times P(n)=f(n /\langle n\rangle)
$$

where $P(n)$ is the probability of generating $n$ hadrons and $f(z)$ is the Levy function $^{2}(z=n /\langle n\rangle)$ with a parameter $c$ that depends on the initial state. Fig.5.2 shows the KNO scaling distributions for $\nu p$ (left) and $\nu n$ interactions. We fit the data points to the Levy function and the best fit parameters are $c=7.93 \pm 0.34$ for the $\nu p$ interactions and $c=5.22 \pm 0.15$ for the $\nu n$ interactions.

- Generate hadrons up to the generated hadron multiplicity taking into account the hadron shower charge conservation and the kinematic constraints. The hadronic system contains any number of mesons and exactly one baryon which is generated based on simple quark model arguments (giving an average number of protons equal to $2 / 3(\nu p), 1 / 2(\nu n), 1 / 2(\bar{\nu} p)$ and $1 / 3(\bar{\nu} n))$. The mesons are generally generated in pairs. The probabilities for pair creation are $30 \%\left(\pi^{0}, \pi^{0}\right)$, $60 \%\left(\pi^{+}, \pi^{-}\right), 2.5 \%\left(K^{0}, K^{-}\right), 2.5\left(\%\left(K^{+}, K^{-}\right), 2.5 \%\left(\bar{K}^{0}, K^{+}\right)\right.$and $2.5 \%\left(K^{0}, \bar{K}^{0}\right)$. If the hadronic invariant mass is only sufficient to create a single pion, a $\pi^{+}$or

\footnotetext{
${ }^{2}$ The Levy function: $\operatorname{Levy}(z ; c)=2 e^{-c} c^{c z+1} / \Gamma(c z+1)$
} 

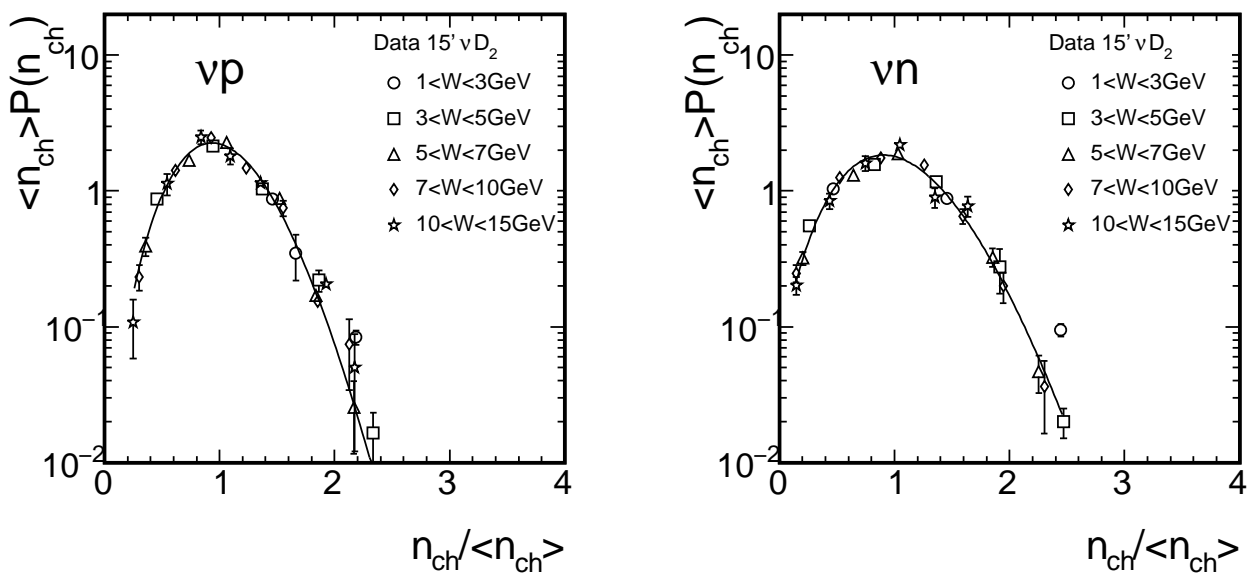

Figure 5.2: KNO scaling distributions for $\nu p$ (left) and $\nu n$ interactions. The curve represents a fit to the Levy function. Data points are taken from [128].

$\pi^{-}$or $\pi^{0}$ is created depending on the total charge of the hadronic system and the baryon selection.

TABLE 5.1 shows the default configuration parameters used in the AGKY model.

\begin{tabular}{rrrrr}
\hline & $\nu p$ & $\nu n$ & $\bar{\nu} p$ & $\bar{\nu} n$ \\
\hline$a$ & $0.40[128]$ & $-0.20[128]$ & $0.02[129]$ & $0.80[129]$ \\
$b$ & $1.42[128]$ & $1.42[128]$ & $1.28[129]$ & $0.95[129]$ \\
$c$ & $7.93[128]$ & $5.22[128]$ & 5.22 & 7.93 \\
\hline
\end{tabular}

Table 5.1: Default AGKY configuration parameters (see text for details)

\subsubsection{Low- $W$ model: Hadron system decay}

Once an acceptable particle content has been generated, the available invariant mass needs to be partitioned amongst the generated hadrons (the so called hadron system decay). The most pronounced kinematic feature in the low- $W$ region is the factor that the produced baryon is heavier than the mesons and exhibits a strong directional anticorrelation with the current direction. 
Our strategy, therefore, would be to correctly reproduce the final state nucleon momentum, and then perform a phase space decay on the remnant system employing, in addition, a phase space decay weighting scheme designed to reproduce the expected meson transverse momentum distribution.

In more detail, the algorithm for decaying a system of $N$ hadrons is the following ${ }^{3}$ :

- Generate the baryon 4-momentum $P_{N}^{*}=\left(E_{N}^{*}, \mathbf{p}_{N}^{*}\right)$ using the nucleon $p_{T}^{2}$ and $x_{F}$ PDFs which are parametrized, based on experimental data shown in Fig.5.3 along with the AGKY best fit parametrization and an error envelope corresponding to $1 \sigma$ variation of the free parameters. We do not take into account the correlation between $p_{T}$ and $x_{F}$ in our selection.
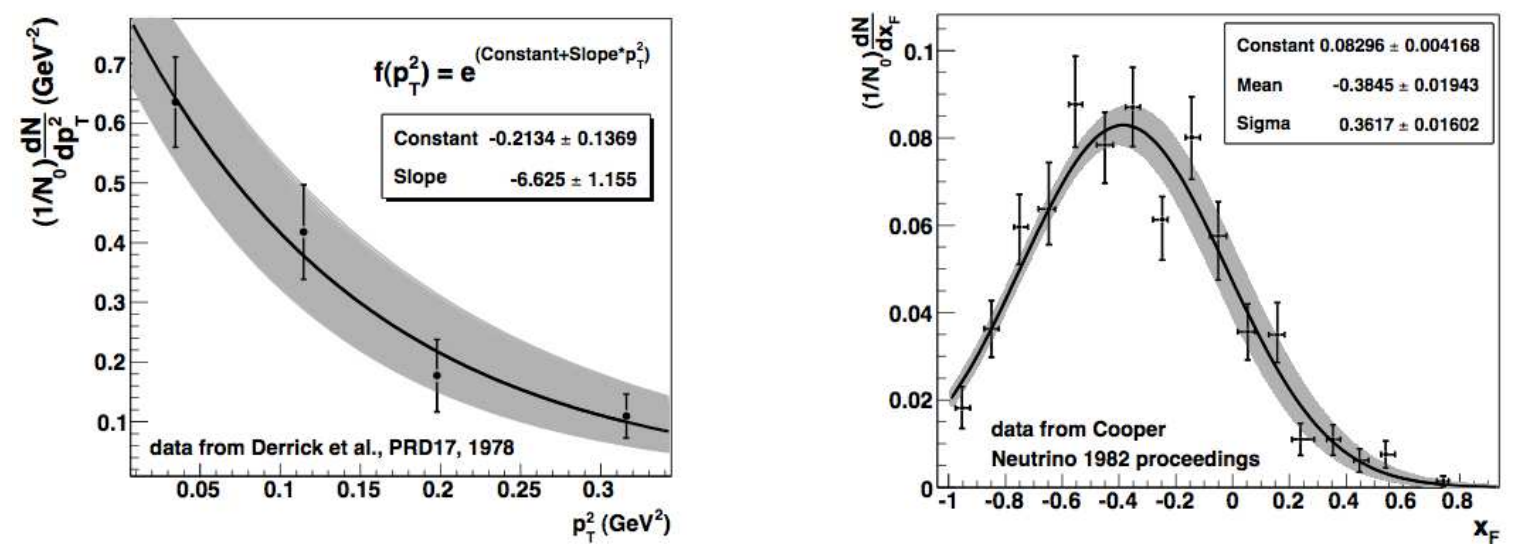

Figure 5.3: Nucleon $p_{T}^{2}$ distribution data from Derrick et al. [121] (left) and nucleon $x_{F}$ distribution data from Cooper et al. [122] (right). The solid lines show the AGKY parametrization, while the shaded area is the error envelope for the $1 \sigma$ variation on its free parameters.

- Once an accepted $P_{N}^{*}$ has been generated, calculate the 4-momentum of the

\footnotetext{
${ }^{3}$ The hadronization model performs its calculation in the hadronic c.m.s., where $\hat{z}:=\mathbf{q}(\mathbf{q}$ is the direction of the momentum transfer). Typically, once the hadronization is completed, the hadronic system will be boosted and rotated to the LAB frame. The boost and rotation maintains the $p_{T}$ generated in the hadronic c.m.s.
} 
remaining N-1 hadrons ("remnant" hadronic system) as $P_{R}^{*}=P_{X}^{*}-P_{N}^{*}$ where $P_{X}^{*}=(W, 0)$ is the initial hadron shower 4-momentum in the hadronic c.m.s.

- Generate an unweighted phase space decay of the remnant hadronic system [123]. The decay takes place at the remnant system c.m.s. and then the particles are boosted back to the hadronic c.m.s. The phase space decay employs the reweighting scheme suggested in [124], readjusting each phase space decay weight including an additional factor $e^{-A * p_{T}}$ for each meson. This improves the agreement of $\left\langle p_{T}>\right.$ distributions between MC predictions and bubble chamber experiment measurements.

2-body hadronic system is a special case where the phase space decay is performed isotropically in the hadronic c.m.s. and no $p_{T^{-}}$based suppression fractor is applied. This is consistent with the fact that the resonance decay is generally isotropic.

\subsubsection{High- $W$ model: PYTHIA/JETSET}

The high invariant mass hadronization is performed by the PYTHIA/JETSET model [105]. The PYTHIA program is a standard tool for the generation of high-energy collisions, comprising a coherent set of physics models for the evolution from a fewbody hard process to a complex multihadronic final state. It contains a library of hard processes and models for initial- and final-state parton showers, multiple partonparton interactions, beam remnants, string fragmentation and particle decays. The hadronization model in PYTHIA is based solely on the Lund string fragmentation framework [125]. In the AGKY model, all but four of the PYTHIA configuration parameters are set to be the default values. Those four parameters take the nondefault values tuned by NUX [126], a high energy neutrino MC generator used by the NOMAD experiment:

- $P_{s \bar{s}}$ controlling the $s \bar{s}$ production suppression (PARJ(2)): 0.21 
- $P_{\left\langle p_{T}^{2}\right\rangle}$ determining the average hadron $\left\langle p_{T}^{2}\right\rangle,(\operatorname{PARJ}(21)): 0.44$

- $P_{n g t}$ parameterizing the non-gaussian $p_{T}$ tails $(\operatorname{PARJ}(23)): 0.01$

- $P_{E c}$ setting an energy cutoff for the fragmentation process (PARJ(33)): 0.20

\subsection{Data/MC Comparisons}

The characteristics of neutrino-produced hadronic systems have been extensively studied by several bubble chamber experiments. The bubble chamber was invented in the early 1950s and became the principal tool of experimental elementary-particle physics in the 1960s and 1970s. A bubble chamber uses a liquid such as liquid hydrogen, deuterium, neon, or Freon, which is put in a superheated state, usually by expanding the liquid to reduce the pressure. Charged particles passing through this liquid leave tracks of tiny bubbles, which are photographed. Most bubble chambers are operated within a magnet so that the momentum of particles can be measured from the curvature of their tracks.

Bubble chambers were used with pulsed beams from particle accelerators and synchronized so that the chamber was expanded right before the particles passed through. This still gave rise to a large background from the tracks left by beam particles which passed through the chamber without a nuclear interaction. Many years after they had been generally superseded for most purposes by fast detectors with an electronic read-out, bubble chambers were still used as a means to investigate the interactions of high energy neutrino beams. In this case there is no background from beam neutrinos since these do not interact significantly in the chamber liquid and the neutrino interactions which do occur are usually dramatic and easy to recognize $[127]$.

The bubble chamber technique is well suited for studying neutrino interactions. 
The bubble chamber can provide precise information on each individual track, which is very useful for building the hadronization model. However, the bubble chamber has disadvantages. Particle identification, essentially based on the track ionization density, was poor, and so was, in case of $H_{2}$ and $D_{2}$ fillings, the detection of neutral particles, in particular of photons from $\pi^{0}$ decay. Since the radiation length is so long, it is difficult to convert photons resulting from $\pi^{0}$ decays inside a hydrogen or deuterium bubble chamber. Several experiments measured $\pi^{0}$ 's using heavy liquids such as neon-hydrogen mixture and Freon.

We tried to distill the vast literature and focus on the following aspects of $\nu / \bar{\nu}$ measurements made in three bubble chambers - the Big European Bubble Chamber (BEBC) at CERN, the 15-foot bubble chamber at Fermilab, and the SKAT bubble chamber in Russia.

We focus on the following quantities measured in the bubble chamber experiments:

- Particle multiplicities.

- Averaged charged and neutral particle $\left(\pi^{0}\right)$ multiplicities.

- Forward and backward hemisphere average multiplicities and correlations.

- Neutral - charged pion multiplicity correlations.

- Characteristics of the hadronic system

- Fragmentation functions ( $z$ distributions).

$-x_{F}$ distributions.

- $p_{T}^{2}$ (transverse momentum squared) distributions.

$-x_{F}-\left\langle p_{T}^{2}\right\rangle$ correlations ("seagull" plots).

The systematic errors in those measurements are quite large and various corrections had to be made to correct for muon selection efficiency, neutrino energy smearing, etc. 
The direction of the incident $\nu / \bar{\nu}$ is well known from the geometry of the beam and the position of the interaction point. Its energy is unknown and is usually estimated using a method based on transverse momentum imbalance. The muon is usually identified through the kinematic information or by using an external muon identifier (EMI). The resolution in neutrino energy is typically $10 \%$ in the bubble chamber experiments and the invariant hadronic mass $W$ is less well determined.

In the QPM (quark-parton model), the differential cross section for semi-inclusive pion production in neutrino interactions

$$
\nu+N \rightarrow \mu^{-}+\pi+X
$$

may be expressed in terms of the elementary scattering of the exchanged current on a constituent quark $\left(q^{\prime}\right)$ followed by the fragmentation of the produced quark $(q)$ into hadrons $(h)$, each of which takes a fraction $z$ of the quark energy:

$$
\frac{d \sigma\left(x, Q^{2}, z\right)}{d x d Q^{2} d z}=\sum\left[\frac{d \sigma\left(x, Q^{2}\right)}{d x d Q^{2}}\right]_{q^{\prime} \rightarrow q} D_{q}^{\pi}(z),
$$

where the sum extends over the different quark flavors taking part. $D_{q}^{\pi}(z)$ is the pion fragmentation function. Experimentally $D_{q}^{\pi}(z)$ is determined as:

$$
D_{q}^{\pi}(z)=\frac{1}{N_{e v}} \cdot \frac{d N(z)}{d z}
$$

where $N_{e v}$ denotes the total number of neutrino interactions and $N$ denotes the number of pions as a function of $z$.

In the framework of the QPM the dominant mechanism for reactions (5.5) is the interaction of the exchanged $W$ boson with a d-quark to give a u-quark which fragments into hadrons in neutrino interactions, leaving a di-quark spectator system 
which produces target fragments. In this picture the fragmentation function is independent of $x$ and the scaling hypothesis excludes a $Q^{2}$ dependence; therefore the fragmentation function should depend only on $z$. There is no reliable way to separate the current fragmentation region from the target fragmentation region if the effective mass of the hadronic system $(W)$ is not sufficiently high. Most experiments required $W>W_{0}$ where $W_{0}$ is between $3 \mathrm{GeV} / c^{2}$ and $4 \mathrm{GeV} / c^{2}$ when studying the fragmentation characteristics. The caused difficulties in the tuning of our model because we are mostly interested in the interactions at low hadronic invariant masses (Fig.5.1).

Some experiments required $Q^{2}>1 \mathrm{GeV}^{2}$ to reduce the quasi-elastic contribution, $y<0.9$ to reduce the neutral currents, and $x>0.1$ to reduce the sea-quark contribution. They often applied a cut on the muon momentum to select clean CC events.

There is one subtlety of the neutrino measurements using bubble chambers which is related to the treatment of proton mis-identification [138]. A proton is distinguishable from a $\pi^{+}$in a bubble chamber only when it is non-relativistic $(\mathrm{P}<1 \mathrm{GeV} / c)$. A bubble chamber has a uniform magnetic field which is generally parallel to the mean axis of the cameras. This arrangement allows momentum to be determined from track curvature: $p=q B R$, where $q$ is the particle charge, $B$ is the magnetic field and $R$ is the curvature. The Bethe-Bloch formula for the mean rate of ionization loss of charged particle is given by

$$
\frac{d E}{d x}=\frac{4 \pi N_{0} z^{2} e^{4}}{m v^{2}} \frac{Z}{A}\left[\ln \left(\frac{2 m v^{2}}{I\left(1-\beta^{2}\right)}\right)-\beta^{2}\right],
$$

where $m$ is the electron mass, $z$ and $v$ are the charge (in units of $e$ ) and velocity of the particle, $\beta=v / c, N_{0}$ is Avogadro's number, $Z$ and $A$ are the atomic number and mass number of the atoms of the medium, and $x$ is the path length in the medium. 
For low energy particles, the energy loss by ionization along the trajectory goes as

$$
\frac{d E}{d x}=\text { const } \times \frac{1}{\beta^{2}} \sim E^{2} / p^{2}=1+M^{2} / p^{2}
$$

where $M$ is the particle mass. That is, as the track goes non-relativistic, the energy loss depends strongly on the particle mass and the trajectory fit has to take that into account. In this case, protons can be identified by using their range and energy loss information. For relativistic particles:

$$
\frac{d E}{d x} \sim \ln (p / M)
$$

The energy loss depends weakly on the particle mass and can be taken as a constant. In this case, a proton is indistinguishable from a $\pi^{+}$since the ionization does not provide sufficient information on the particle mass. In most bubble chamber experiments, the pion mass is assigned to all charged hadrons if their laboratory momenta exceed $1 \mathrm{GeV} / c$.

In order to validate the AGKY hadronization model, we determined the parameters in our model by fitting experimental data and generated CC neutrino free nucleon interactions uniformly distributed in the energy range from 1 to $61 \mathrm{GeV}$. The quasi-elastic contribution is negligible in our sample. The MC events were analyzed to determine the hadronic system characteristics and compared with some bubble chamber experimental data taken in BEBC, Fermilab 15-foot chamber, and SKAT. We apply the same kinematic cuts as explicitly stated in the papers to our MC events. We reweight our MC to the energy spectrum measured by the experiment if that information is available. But this step is not strictly necessary for the following two reasons: firstly a lot of observables (mean multiplicity, dispersion, etc.) are measured as a function of the hadronic invariant mass $W$, in which case the energy dependency is removed; secondly the scaling variables $\left(x_{F}, z\right.$, etc.) are rather independent of 
energy according to the scaling hypothesis.

Fig.5.4 shows the average charged hadron multiplicity $\left\langle n_{c h}\right\rangle$ (the number of charged hadrons in the final state, i.e. excluding the muon) as a function of $W^{2} .\left\langle n_{c h}\right\rangle$ rises linearly with $\ln \left(W^{2}\right)$ for $W>2 \mathrm{GeV} / c^{2}$. At the lowest $W$ values the dominant interaction channels are the resonance production:

$$
\begin{aligned}
& \nu+p \rightarrow \mu^{-}+p+\pi^{+} \\
& \nu+n \rightarrow \mu^{-}+p+\pi^{0} \\
& \nu+n \rightarrow \mu^{-}+n+\pi^{+}
\end{aligned}
$$

Therefore $\left\langle n_{c h}\right\rangle$ becomes 2(1) for $\nu p(\nu n)$ interactions as $W$ approaches the pion production threshold. For $\nu p$ interactions there is a disagreement between the BEBC and FNAL measurements especially at high invariant masses. The reason may be that the Fermilab measurement used deuterium as the target while the BEBC measurement used hydrogen as the target. Our parameterization of low- $W$ model was based on the Fermilab 15-foot chamber data. Historically the PYTHIA/JETSET program was tuned on the BEBC data. The AGKY model uses the KNO-based empirical model at low invariant masses and it uses the PYTHIA/JETSET program to simulate high invariant mass interactions. Therefore the MC prediction agrees better with the Fermilab data at low invariant masses and it agrees better with the BEBC data at high invariant masses.

Fig.5.5(a) shows the dispersion $D_{-}=\left(\left\langle n_{-}^{2}\right\rangle-\left\langle n_{-}\right\rangle^{2}\right)^{1 / 2}$ of the negative hadron multiplicity as a function of $\left\langle n_{-}\right\rangle$. Fig.5.5(b) shows the ratio $D /\left\langle n_{c h}\right\rangle$ as a function of $W^{2}$. The dispersion is solely determined by the KNO scaling distributions shown in Fig.5.2. The agreement between data and MC predictions is satisfactory.

Fig.5.6(a) shows the average $\pi^{0}$ multiplicity $\left\langle n_{\pi^{0}}\right\rangle$ as a function of $W^{2}$. Fig.5.6(b) shows the dispersion of the distributions in multiplicity as a function of the average 

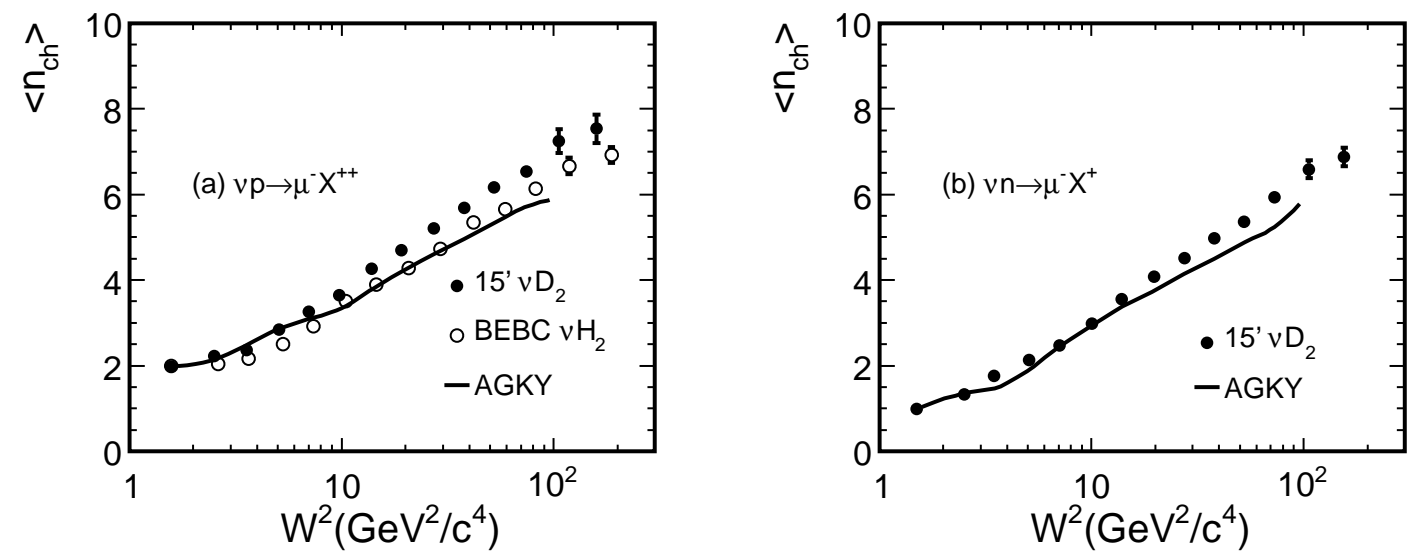

Figure 5.4: Average charged-hadron multiplicity $\left\langle n_{c h}\right\rangle$ as a function of $W^{2}$. (a) $\nu p$ events. (b) $\nu n$ events. Data points are taken from $[128,130]$.
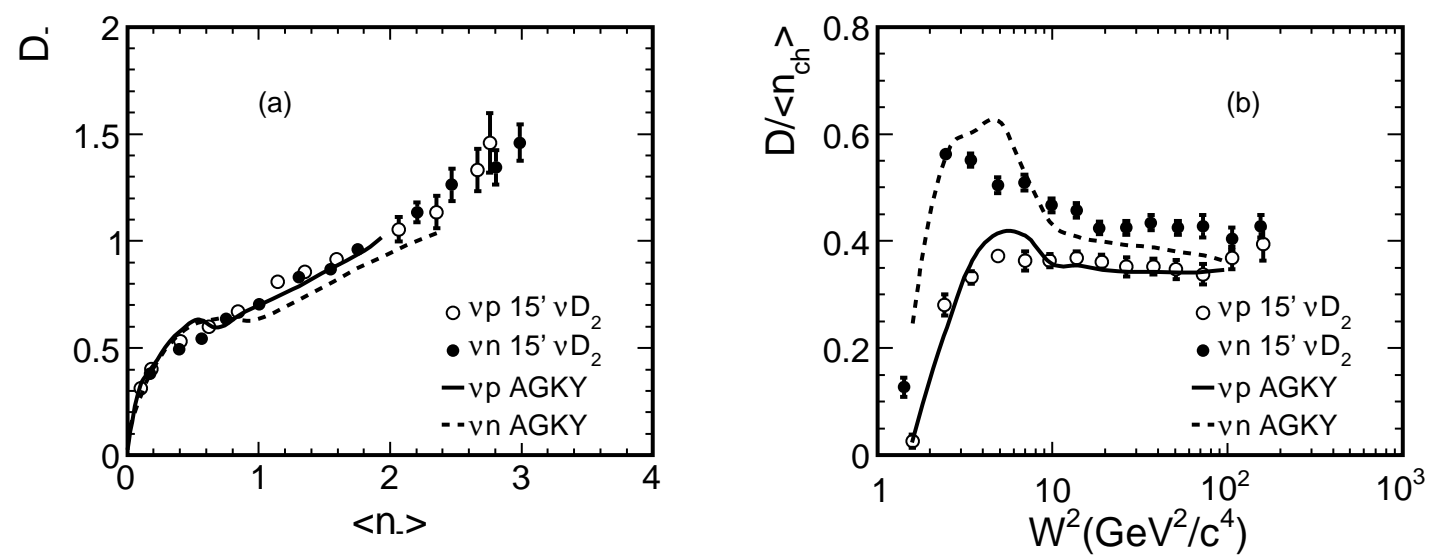

Figure 5.5: (a) The dispersion $D_{-}=\left(\left\langle n_{-}^{2}\right\rangle-\left\langle n_{-}\right\rangle^{2}\right)^{1 / 2}$ as a function of $\left\langle n_{-}\right\rangle$. $D /\left\langle n_{c h}\right\rangle$ as a function of $W^{2}$. Data points are taken from [128]. 
multiplicity of $\pi^{0}$ mesons. As we mentioned it is difficult to detect $\pi^{0}$ 's inside a hydrogen bubble chamber. Also shown in the plot are some measurements using heavy liquids such as neon and Freon. In principle, rescattering of the primary hadrons can occur in the nucleus. Some studies of inclusive negative hadron production in the hydrogen-neon mixture and comparison with data obtained by using hydrogen targets indicate that these effects are negligible [136]. The MC is in good agreement with the data. $\left\langle n_{\pi^{0}}\right\rangle$ is $0(1 / 2)$ for $\nu p(\nu n)$ interactions when the hadronic invariant mass gets down to the resonance production threshold, which is consistent with the expectation from the reactions $(5.11-5.13)$. The MC predicts the same average $\pi^{0}$ multiplicity for $\nu p$ and $\nu n$ interactions for $W>2 \mathrm{GeV} / c^{2}$.
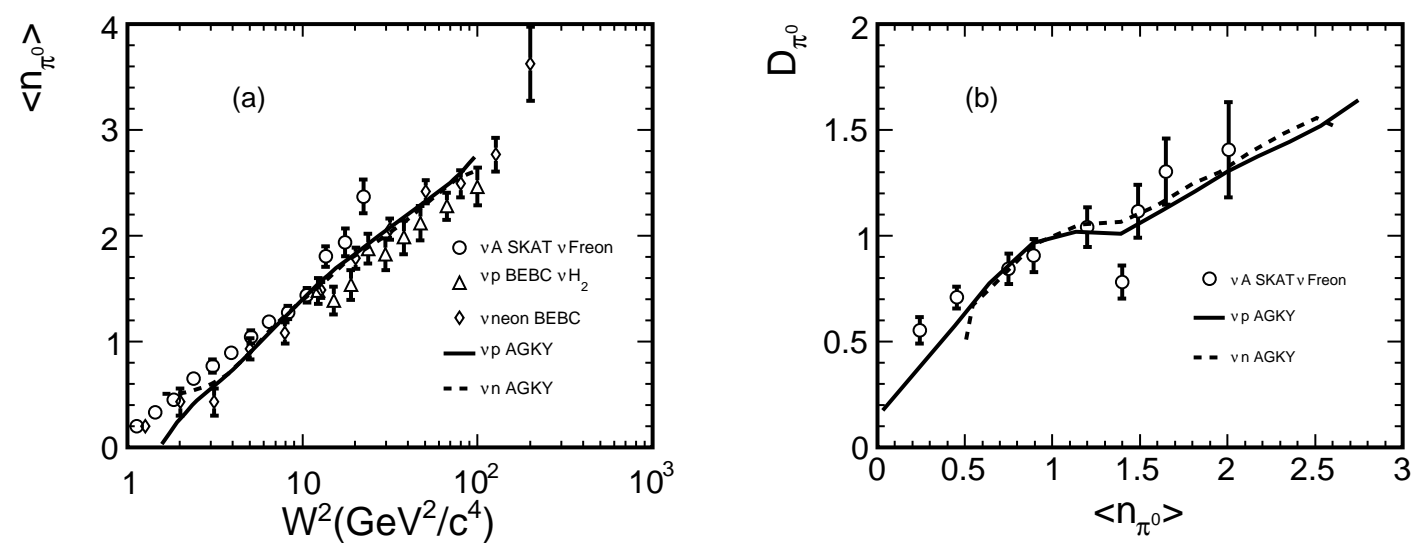

Figure 5.6: (a) Average multiplicity of $\pi^{0}$ mesons as a function of $W^{2}$. (b) Dispersion of the distributions in multiplicity as a function of the average multiplicity of $\pi^{0}$ mesons. Data points are taken from [119, 131, 132]

Fig.5.7 shows the average $\pi^{0}$ multiplicities $\left\langle n_{\pi^{0}}\right\rangle$ as a function of the number of negative hadrons $n_{-}$for various $W$ ranges. At lower $W,\left\langle n_{\pi^{0}}\right\rangle$ tends to decrease with $n_{-}$, probably because of limited phase space, while at higher $W\left\langle n_{\pi^{0}}\right\rangle$ is rather independent of $n_{-}$where there is enough phase space. Our MC was tuned to reflect the correlation at lower $W$ suggested by the data. However, another experiment 
measured the same correlation using neon-hydrogen mixture and their results indicate that $\left\langle n_{\pi^{0}}\right\rangle$ is rather independent of $n_{-}$for both $W>4 \mathrm{GeV} / c^{2}$ and $W<4 \mathrm{GeV} / c^{2}$ [133]. Since events with $\pi^{0}$ but with 0 or very few charged pions are dominant background events in the $\nu_{e}$ appearance analysis, it is very important to understand the correlation between the neutral pions and charged pions. Lack of knowledge of this correlation is a shortcoming of our current MC, which will be improved when the relevant measurements are available in the future.

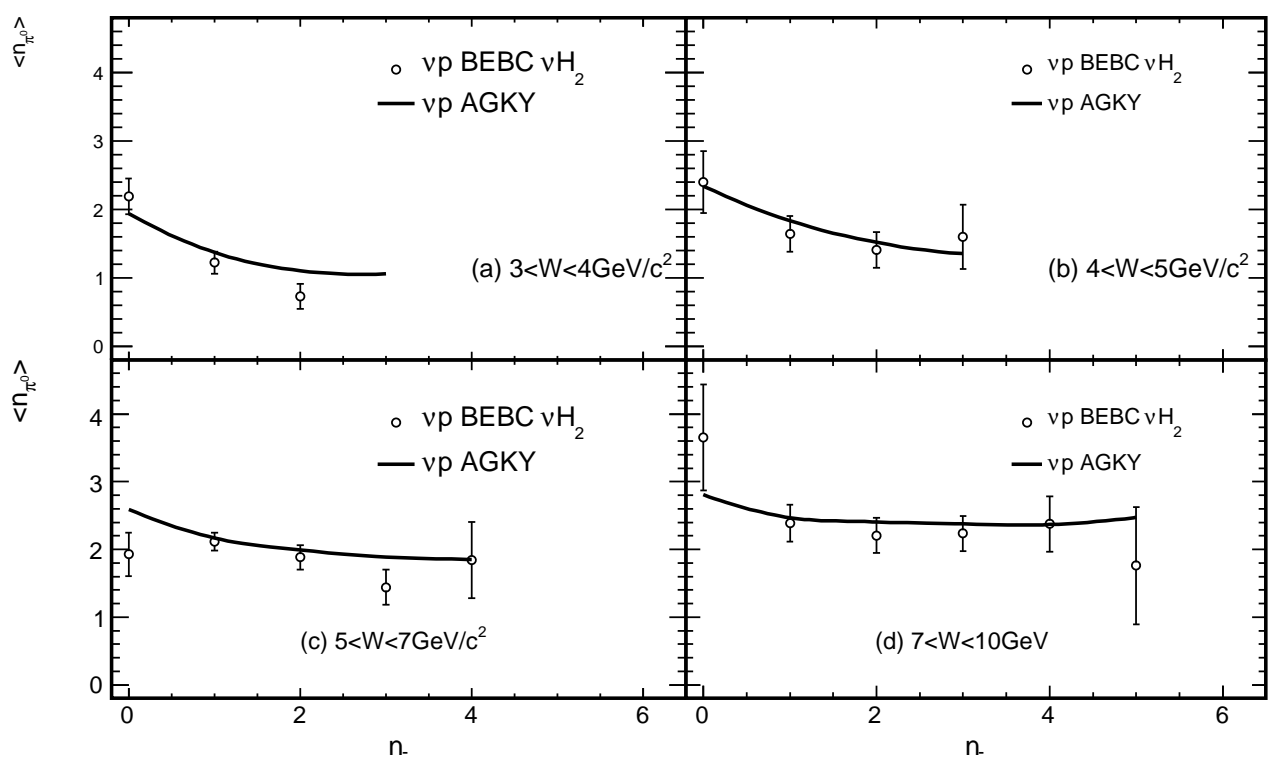

Figure 5.7: Average $\pi^{0}$ multiplicity $\left\langle n_{\pi^{0}}\right\rangle$ as a function of the number of negative hadrons $n_{-}$for different intervals of $W$. Data points are taken from [132].

Fig.5.8 shows the average charged-hadron multiplicity in the forward and backward hemispheres as functions of $W^{2}$. The forward hemisphere is defined by the direction of the current in the total hadronic c.m.s. There is a rather big disagreement between the Fermilab 15-foot chamber and BEBC measurements. There is a bump in the $\mathrm{MC}$ prediction in the forward hemisphere for $\nu p$ interactions at $W \sim 2 \mathrm{GeV} / c^{2}$ 
and there is a slight dip in the backward hemisphere in the same region. This indicates that the MC may overestimate the hadrons going forward in the hadronic c.m.s. at $W \sim 2 \mathrm{GeV} / c^{2}$ and underestimate the hadrons going backward. One consequence could be that the $\mathrm{MC}$ overestimates the energetic hadrons since the hadrons in the forward hemisphere of hadronic c.m.s. will have higher energy after being boosted to the LAB frame than those in the backward hemisphere. This may be caused by the way we determine the baryon 4-momentum and preferably select events with low $p_{T}$ in the phase space decay.

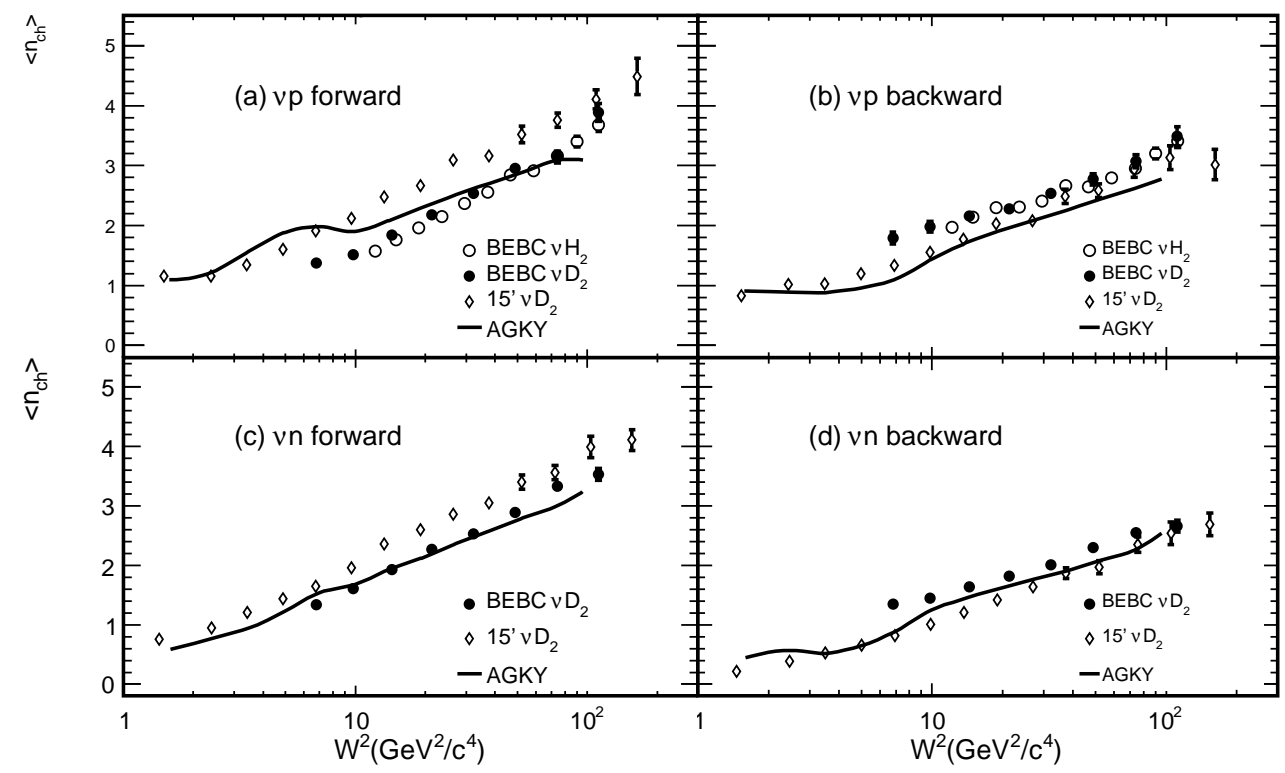

Figure 5.8: Average charged-hadron multiplicity in the forward and backward hemispheres as functions of $W^{2}$ : (a) $\nu p$, forward, (b) $\nu p$, backward, (c) $\nu n$, forward, (d) $\nu n$, backward. Data points are taken from $[128,132,134]$.

Fig.5.9 shows the fragmentation functions for positive and negative hadrons. The fragmentation function is defined as: $D(z)=\frac{1}{N_{e v}} \cdot \frac{d N}{d z}$, where $N_{e v}$ is the total number of interactions (events) and $z=E / \nu$ is the fraction of the total energy transfer carried by each final hadron in the laboratory frame. The AGKY predictions are in excellent agreement with the data. 

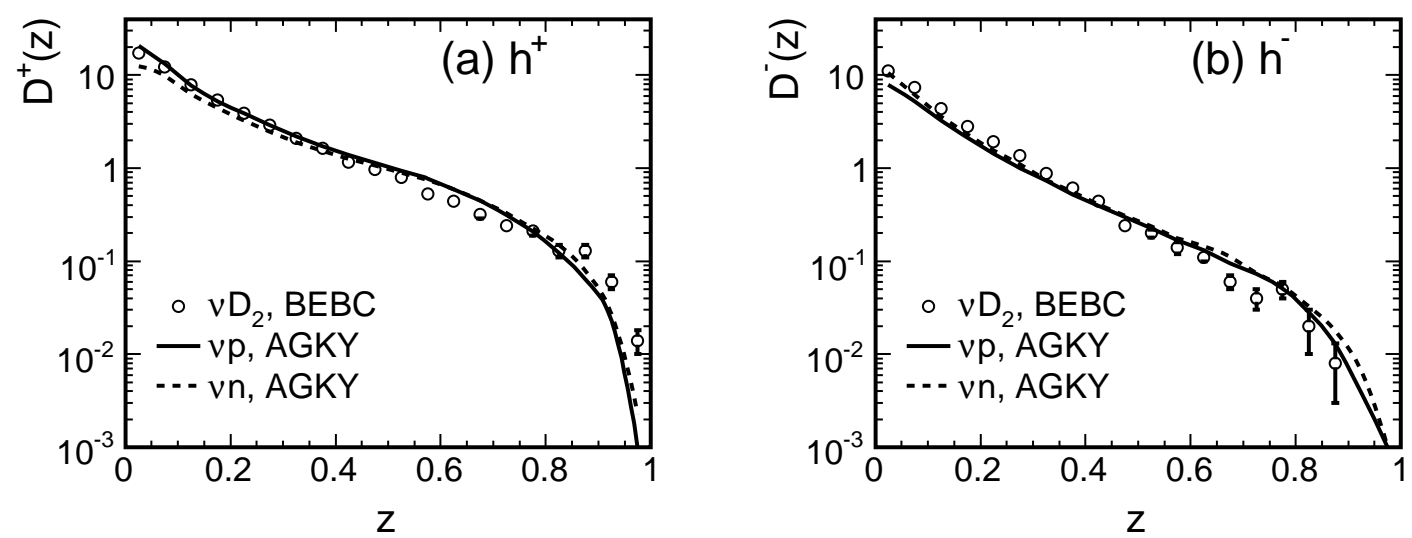

Figure 5.9: Fragmentation functions for positive (a) and negative (b) hadrons. Applied cuts: $W^{2}>5\left(G e V / c^{2}\right)^{2}, Q^{2}>1(G e V / c)^{2}$. Data points are taken from [134].

Fig.5.10 shows the mean value of the transverse momentum (with respect to the current direction) of charged hadrons as a function of $W$. The MC predictions match the data pretty well. In the naive QPM, the quarks have no transverse momentum within the struck nucleon, and the fragments acquire a $P_{T}^{\text {frag }}$ with respect to the struck quark from the hadronization process. The average transverse momentum $\left\langle P_{T}^{2}\right\rangle$ of the hadrons will then be independent of variables such as $x_{B J}, y, Q^{2}, W$, etc., apart from trivial kinematic constraints and any instrumental effects. Both MC and data reflect this feature. However, in a perturbative QCD pircture, the quark acquires an additional transverse component, $\left\langle P_{T}^{2}\right\rangle^{Q C D}$, as a result of gluon processes. The quark itself may also have a primordial $\left\langle P_{T}^{2}\right\rangle^{\text {prim }}$ inside the nucleon. The QCD effect can introduce dependencies of $\left\langle P_{T}^{2}\right\rangle$ on the variables $x_{B J}, y, Q^{2}, W$, z, etc.

Fig.5.11 shows the mean value of the transverse momentum of charged hadrons as a function of $x_{F}$, where $x_{F}=\frac{p_{L}^{*}}{p_{L m a x}^{*}}$ is the Feynman-x. As is well known, $\left\langle p_{T}\right\rangle$ increases with increasing $\left|x_{F}\right|$ with a shape called the seagull effect. This effect is reasonably well modeled by the AGKY model. 

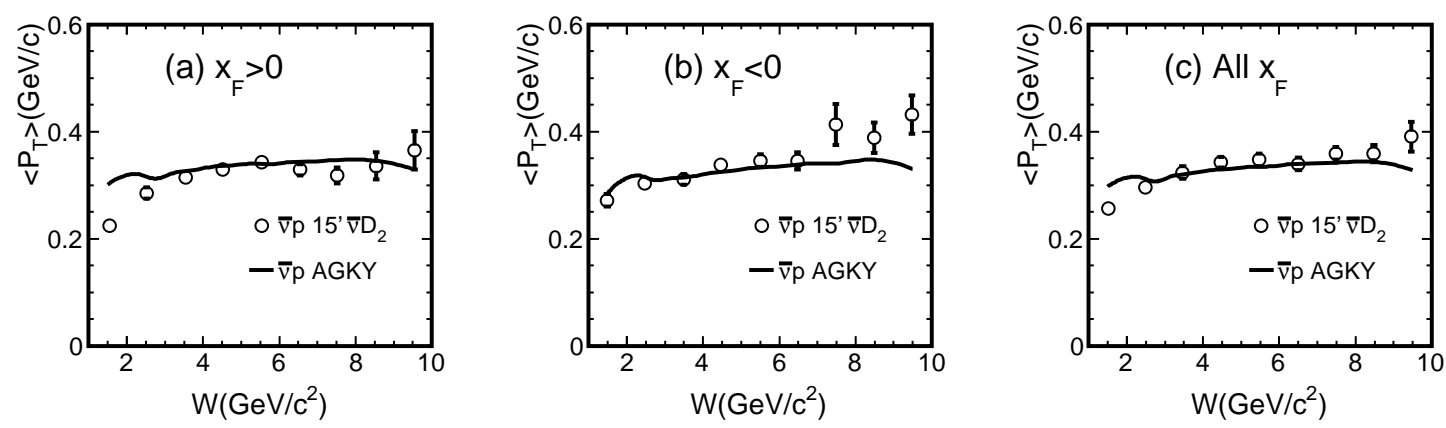

Figure 5.10: Mean value of the transverse momentum of charged hadrons as a function of $W$ for the selections (a) $x_{F}>0$, (b) $x_{F}<0$, and (c) all $x_{F}$. Data points are taken from [137].
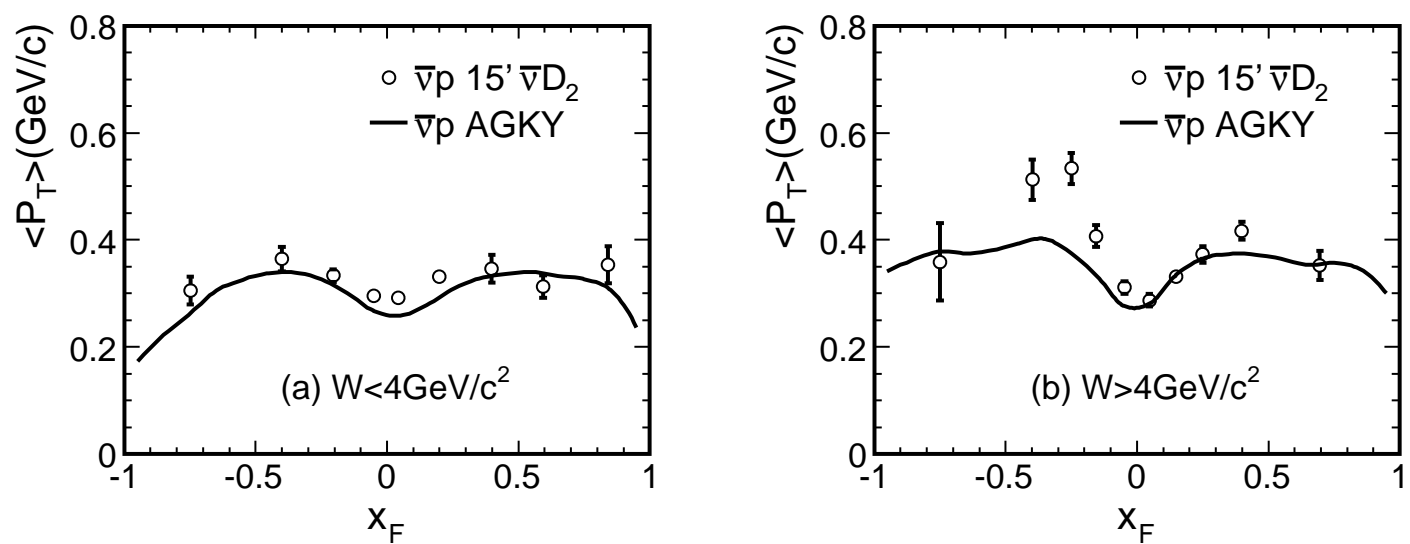

Figure 5.11: Mean value of the transverse momentum of charged hadrons as a function of $x_{F}$ for $\bar{\nu} p$. (a) $W<4 \mathrm{GeV} / c^{2}$, (b) $W>4 \mathrm{GeV} / c^{2}$. Data points are taken from [137]. 


\section{$5.4 \quad$ Intranuclear Rescattering}

The AGKY hadronization model reasonably well describes the hadron production in the neutrino-nucleon interactions. However the MINOS detectors are steel-scintillator calorimeters. Most of the neutrino interactions occur in the steel planes where nucleons are bound inside iron nuclei of significant spatial extent; hence, final-state hadrons resulting from neutrino-nucleon interactions may reinteract with other nucleons within the same nucleus before emerging.

Hadron intranuclear rescattering may involve any of the four processes which occur in low energy hadron-nucleus scattering.

- Absorption, in which the hadron "disappears" within the nucleus with resultant energy transfer to the constituent nucleons.

- Charge exchange, in which a charged (neutral) hadron changes into a neutral (charged) hadron; the (re)scattered hadron may be deflected and momentumdegraded relative to the initial hadron.

- Inelastic (re)scattering, in which the hadron's identity is maintained but its momentum is degraded; the hadron is deflected, possibly to large angles.

- Elastic (re)scattering; the hadron retains its original entity and energy, but is deflected.

Each of the processes has an associated total cross section - $\sigma_{\text {elas }}$ for elastic scattering, $\sigma_{\text {inel }}$ for inelastic scattering, $\sigma_{c e x}$ for single charge exchange, and $\sigma_{a b s}$ for absorption. The total cross section $\left(\sigma_{t o t}\right)$ is the sum of all component cross sections and the total reaction cross section $\left(\sigma_{\text {reac }}\right)$ is the sum of all inelastic reactions,

$$
\sigma_{\text {reac }}=\sigma_{\text {cex }}+\sigma_{\text {inel }}+\sigma_{\text {abs }}=\sigma_{\text {tot }}-\sigma_{\text {elas }}
$$


The data for $\sigma_{\text {tot }}, \sigma_{\text {reac }}$ (pions) and $\sigma_{\text {reac }}$ (protons) are of high quality over a broad range of incident energies and many targets. The component cross sections are only known at somewhat lower energies and with larger uncertainties because separation of final states is difficult.

In the neutrino generator NEUGEN, hadron intranuclear rescattering is handled by the program INTRANUKE, which was originally developed by many people, especially Tony Mann and Robert Merenyi of Tufts University [139]. It was further developed by Steven Dytman and Hugh Gallagher [140]. The treatment of hadrons in INTRANUKE uses a semiclassical model (intranuclear cascade model - INC). During the time (referred to as formation time) it takes for the quarks to materialize as hadrons, they propagate through the nucleus with a dramatically reduced interaction probability. This was implemented in INTRANUKE as a simple "free step" at the start of the intranuclear cascade during which no interactions can occur. The formation time is the only free parameter and has the same value of $0.342 \mathrm{fm} / \mathrm{c}$ as in the SKAT model [141]. After the quark is fully materialized, the probability of the resulting hadrons to interact along their path in the nucleus (target) is governed by the usual exponential law with the mean free path for a given energy given by:

$$
\lambda(E, r)=\frac{1}{\rho(r) \sigma_{t o t}(E)}
$$

where $\rho$ is the nuclear matter density, $\sigma_{t o t}$ is the total hadron-nucleus cross section, $E$ is the incident hadron energy, and $r$ is the radial position of the hadron. If a scattering occurs, the choice of final state is made in proportion to the component total cross section taken from data. For each final state, the outgoing particles and their 4-momenta are then determined with input from data and kinematic constraints. 


\subsection{Conclusions}

The AGKY hadronic multiparticle production model has been described in detail in this chapter. The model predictions agree reasonably well with a wide variety of measurements on the hadronic system characteristics. Nevertheless additional effort is necessary to fine tune this model in order to improve its performance. Better data focused on the remaining issues in the hadronization model is very important. 


\section{Chapter 6}

\section{Crosstalk Tuning}

\subsection{Introduction}

The MINOS $\nu_{e}$ selection algorithm distinguishes the $\nu_{e}$ signals from background using event topological information as described in Chapter 4. We measure the background rate at the Near Detector and then extrapolate the ND background rate to the FD using Monte Carlo information to obtain the FD background prediction. Therefore it is very important to understand the event topology in the MINOS detectors, especially the differences between the Near and Far Detectors.

An area that is difficult to simulate and may have a large impact on the event topology is the modeling of low pulse-height hits. Fig.6.1 shows the Near Detector strip pulse-height distributions (measured in photo-electrons (PEs)) of events passing the Near Detector fiducial volume cuts (plot on the left) and passing the $\nu_{e}$ preselection cuts which were described in Chapter 4 (plot on the right) compared with the original MC predictions. In order to take into account the temporal variation of PMT gains, both data and MC are sampled from March 2005 to June 2007. In both plots, the MC predictions are in good agreement with the data for hits above 1PE; however, MC seems to largely underestimate the number of hits less than 1PE. The 
mis-modeling of low pulse-height hits was first pointed out by Rustem Ospanov of UT Austin when developing his particle-ID algorithm for the MINOS CC analysis[142].
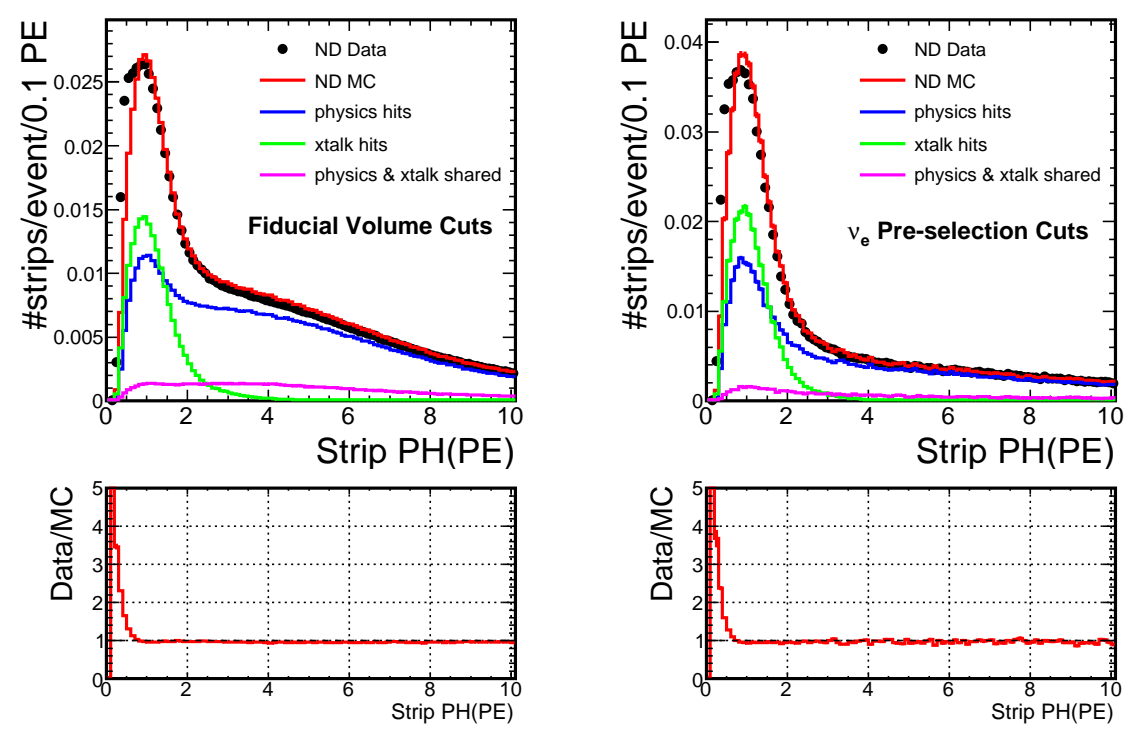

Figure 6.1: Near Detector strip pulse-height distributions (measured in photoelectrons - PEs) of events passing the Near Detector fiducial volume cuts (left) and passing the $\nu_{e}$ pre-selection cuts (right). Different components of MC simulation are also shown. Data and MC are sampled from March 2005 to June 2007 to average the temporal variation of PMT gains.

The causes of the mis-modeling of low pulse-height hits are not completely understood. The current suspects include the imperfect modelings of PMT crosstalk, PMT gains (especially single PE width), and detector noise, etc. Fig.6.1 shows different components of the MC predictions. Approximately half of the hits below $2 \mathrm{PE}$ are crosstalk hits. The excess of low pulse-height hits in the data seems to indicate an underestimate of the crosstalk hits in the MC simulation. It can also be seen from the plots that the single PE width of data is broader than the MC simulation. The data/MC discrepancy may also be caused by the mis-modeling of the PMT single PE width. There are other features of the detector and electronics which are either poorly or not modeled in the MC and may partially account for the mis-modeling of 
low pulse-height hits. These features include the fiber noise, the electronics noise, and the PMT after-pulsing. However there is no clear evidence that they are the main sources of the excess of low pulse-height hits in the data.

It was discovered in early 2008 that the low pulse-height hits have a rather large impact on the MINOS $\nu_{e}$ analysis. Since then a lot of effort has been devoted to understanding the causes of this problem and finding the cure. We identified PMT crosstalk as one of the biggest contributor to the low pulse-height hits. Unfortunately the crosstalk was poorly modeled in the Monte Carlo. We used the cosmic ray data to obtain better understanding of the crosstalk. Based on those studies we decided to make several changes to the analysis including removing low pulse-height hits at the analysis time so as to make the $\nu_{e}$ analysis more immune to any inaccuracies in the crosstalk modeling.

This chapter discusses 1) the impact of the low pulse-height hits on the MINOS $\nu_{e}$ analysis, 2) the attempt to improve the PMT crosstalk model and 3) make the $\nu_{e}$ selection algorithm less sensitive to the low pulse-height hits.

\subsection{Impact on the $\nu_{e}$ Analysis}

The mis-modeling of the low pulse-height hits can affect the event reconstruction, especially the shower reconstruction, and change the event topology, which causes the $\mathrm{MC}$ events to look different than the data events. Therefore it can affect the background estimation and signal selection in the $\nu_{e}$ analysis. Furthermore, if the excess of low pulse-height hits is indeed caused by the mis-modeling of crosstalk hits, this effect can affect the Near Detector and the Far Detector differently since they have different PMT crosstalk patterns, which can introduce a systematic uncertainty in the Far Detector background rate prediction through extrapolation from the Near Detector. In this section, we will discuss the impact of the low pulse-height hits on 
the $\nu_{e}$ analysis by removing them in the reconstruction. In the next section, we will try to identify the causes of the mis-modeling of low pulse-height hits and discuss the attempt to improve the MC simulation.

In order to understand the impact of the low pulse-height hits on the reconstruction and the $\nu_{e}$ analysis, we remove hits whose pulse-heights are less than $2 \mathrm{PE}$ in the reconstruction software. We then reconstructed a fraction of data and MC events using this modified software and compared them with the standard reconstructed events where all hits were used during the reconstruction process. The choice of the 2PE cut is somewhat arbitrary. Fig.6.1 shows that the simulation does not model the single PE peak well. Even though data and MC agree above 1PE, in order to remove the whole single PE peak, we need to cut higher than 1PE. Also we have evidence that the crosstalk is not well modeled in the MC simulation. By placing the cut at $2 \mathrm{PE}$, one can remove the majority of the crosstalk hits in order to reduce their impact on the reconstruction.

Fig.6.2 shows an example of a Near Detector MC event before (left) and after (right) applying the $2 \mathrm{PE}$ cut. The $2 \mathrm{PE}$ cut changes the event topology significantly. Most of the peripheral hits are removed by this cut. Those hits are consistent with the crosstalk hits and they hold very little physics information.

Fig.6.3 shows three reconstructed quantities before and after applying the $2 \mathrm{PE}$ cut. Each sub panel shows three plots. The top plot shows the distributions before and after applying the $2 \mathrm{PE}$ cut. The middle plot shows the ratio of the two distributions. The bottom plot shows the difference in each reconstructed quantity on an event by event basis. 6.3(a) shows the distributions of the track lengths expressed in number of traversed planes. Removing hits less than $2 \mathrm{PE}$ has a negligible effect on the reconstructed track length. This indicates that the track reconstructed is not very sensitive to the low pulse-height hits. 6.3(b) and 6.3(c) show the shower length and reconstructed event energy distributions. The $2 \mathrm{PE}$ cut makes the reconstructed 

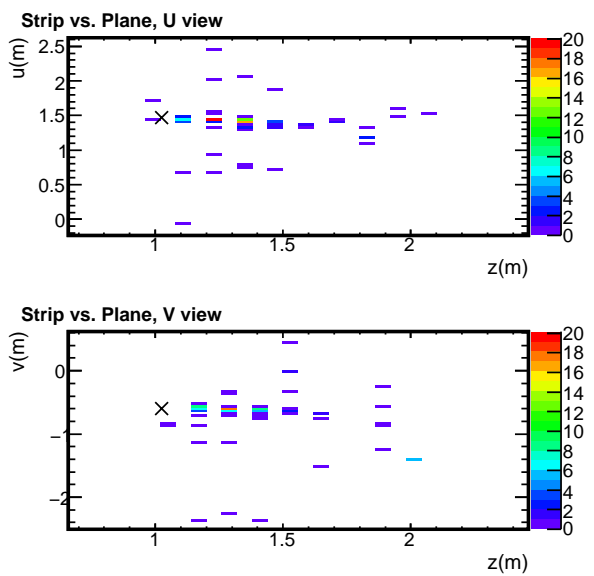

(a) Original Event
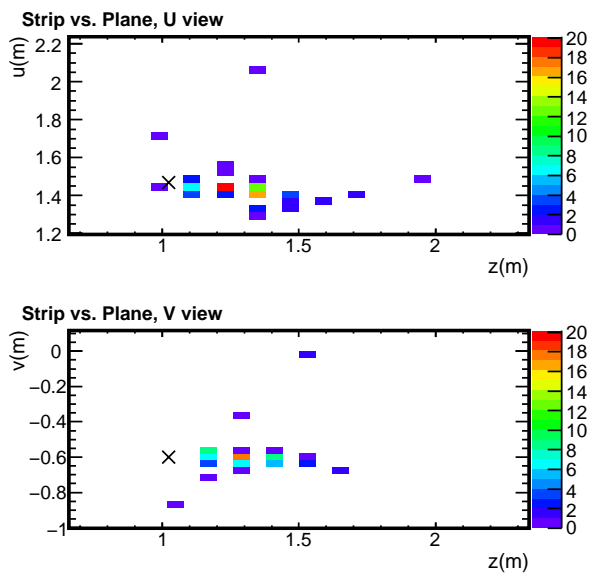

(b) Same event after applying a $2 \mathrm{PE}$ cut

Figure 6.2: Event displays of one Near Detector MC event. (a) shows the standard reconstructed event; (b) shows the same event after removing low pulse-height hits $(<2 \mathrm{PE})$ at the reconstruction level. The cross represents the reconstructed event vertex. The color scale represents the strip pulse-height which is in MIPs.

showers shorter and introduces a $\sim 200 \mathrm{MeV}$ shift in the reconstructed event energy on average. This shift has to be taken into account in the new calibration if we decide to apply the $2 \mathrm{PE}$ cut in the future MINOS reconstruction. A track cut track length $<25$ planes was applied in both figures (b) and (c) to only accept events without a long muon track. The drastic change in the shower length distribution indicates that the $2 \mathrm{PE}$ cut has a rather large impact on the shower topology, thus can affect the $\nu_{e}$ selection algorithm.

Fig.6.4 shows three $\nu_{e}$ analysis variables before (black) and after (red) applying the 2PE cut. 6.4(a) shows the variable shwfit.par_b which describes the longitudinal shower characteristic. 6.4(b) shows the variable shwfit.uv_rms which characterizes the lateral spread ("width") of the shower. The details of those two variables are described in Chapter 4 . It is clear that the $2 \mathrm{PE}$ cut has a larger impact on the transverse shower variable than on the longitudinal shower variable. Removing hits less than 2PE makes the shower consistently narrower. 6.4(c) shows the PID distribution 

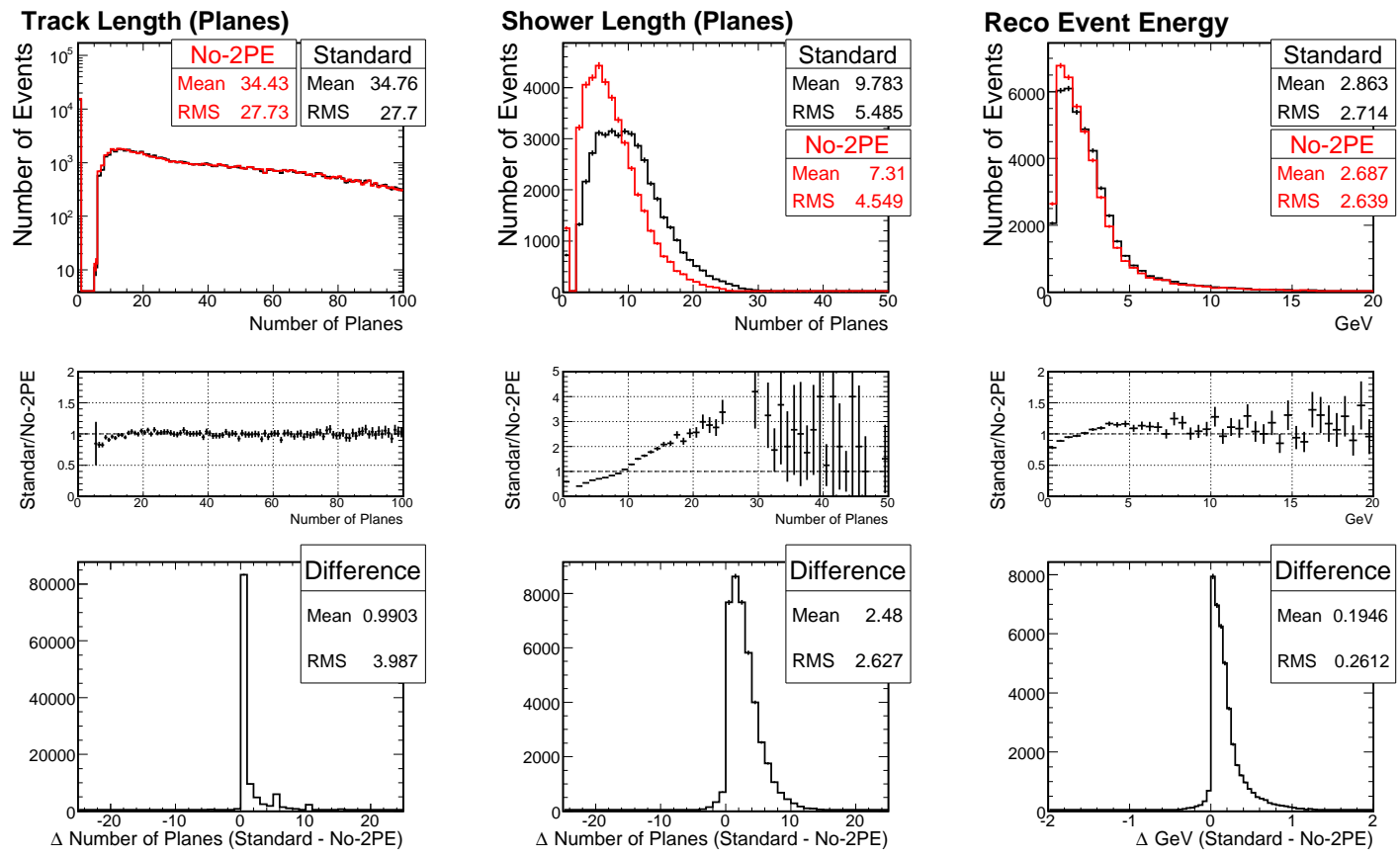

(a) Track Length

(b) Shower Length

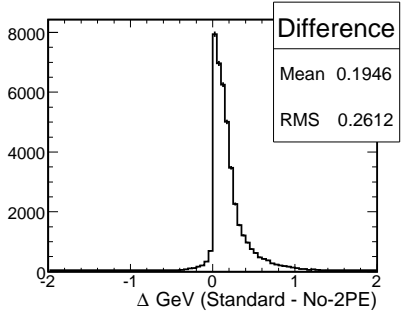

(c) Reco. Event Energy

Figure 6.3: Reconstructed quantities before (black) and after (red) applying the 2PE cut (ND MC). A track cut track length < 25 planes was applied in figures (b) and (c) to only accept events without a long muon track.

for the original version of ANN, before adoption of the modifications discussed below. This ANN is the precursor to the one discussed in Chapter 4. It has 30 input variables and some of the variables (such as shwfit.uv_rms) are quite sensitive to the low pulse-height hits. Applying the $2 \mathrm{PE}$ cut makes the distribution of this ANN PID shift to the right. The transverse shower variables can be modified to be less dependent on the low pulse-height hits. This will be discussed later in this chapter. A new ANN (described in detail in Chapter 4) was constructed based on the modified variables. The variable shwfit.uv_rms plotted here is before any revisions so it is labeled as "unmodified" in the plot.

We have demonstrated that removing hits less than 2PE has a sizable impact on 

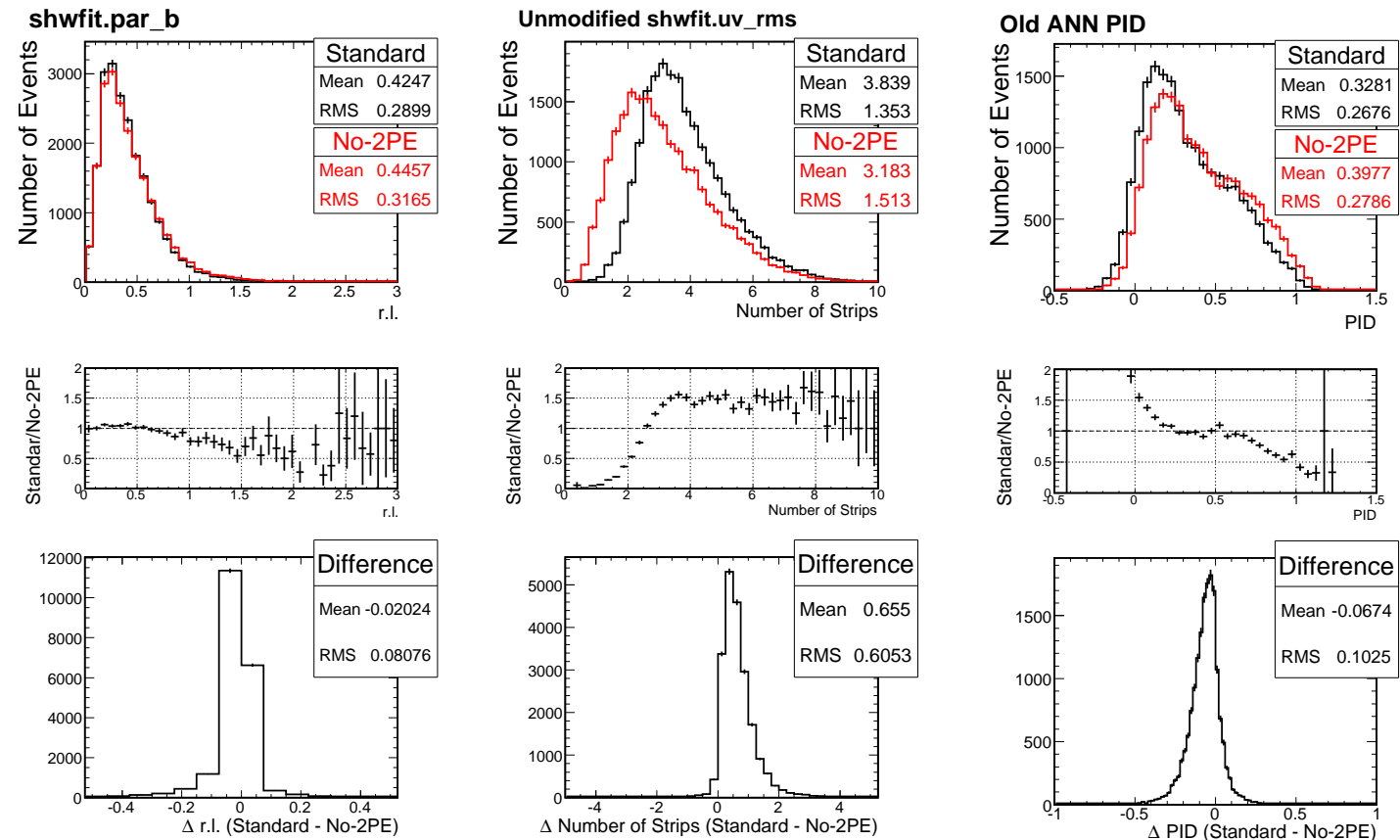

(a) shwfit.par_b

(b) Unmodified shwfit.uv_rms

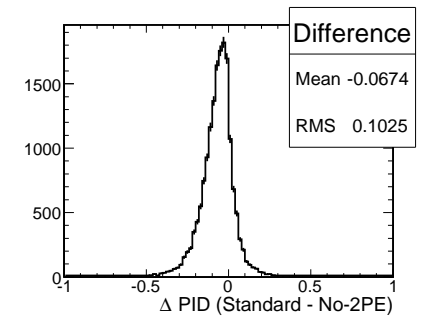

(c) Old ANN PID

Figure 6.4: $\nu_{e}$ analysis variables before (black) and after (red) applying the 2PE cut (ND MC). $\nu_{e}$ pre-selection cuts are applied.

the MC shower topology. Since the MC simulation underestimates the low pulseheight hits in the data, we expect the low pulse-height hits to have a larger impact on data events. These studies showed that we needed to understand the source of the excess of the low pulse-height hits in data to some extent and more importantly, make our selection algorithm less sensitive to the low pulse-height hits.

\subsection{PMT Crosstalk Tuning}

We have demonstrated that the older $\nu_{e}$ event selection algorithm is quite sensitive to the low pulse-height hits since those hits can change event topology drastically. Fig.6.1 shows that the crosstalk hits contribute approximately half of the hits whose pulse-heights are less than 2PE. The mis-modeling of low pulse-height hits seems to 
indicate a mis-modeling of the crosstalk hits. The magnitude of PMT crosstalk was measured at the test stand [143] and used in the Monte Carlo simulation. In this section, we will describe a different approach of measuring the crosstalk using the cosmic ray muon data. This approach takes advantage of the high statistics of cosmic ray muon data at both detectors and is believed to be a more precise measurement of crosstalk.

The MINOS detectors are read out by Hamamatsu 64-anode (M64) PMTs (photomultiplier tubes) for the Near Detector and 16-anode (M16) PMTs for the Far Detector. Fig.6.5 shows the M16 PMT mounting assembly and the face-on fiber "cookie"

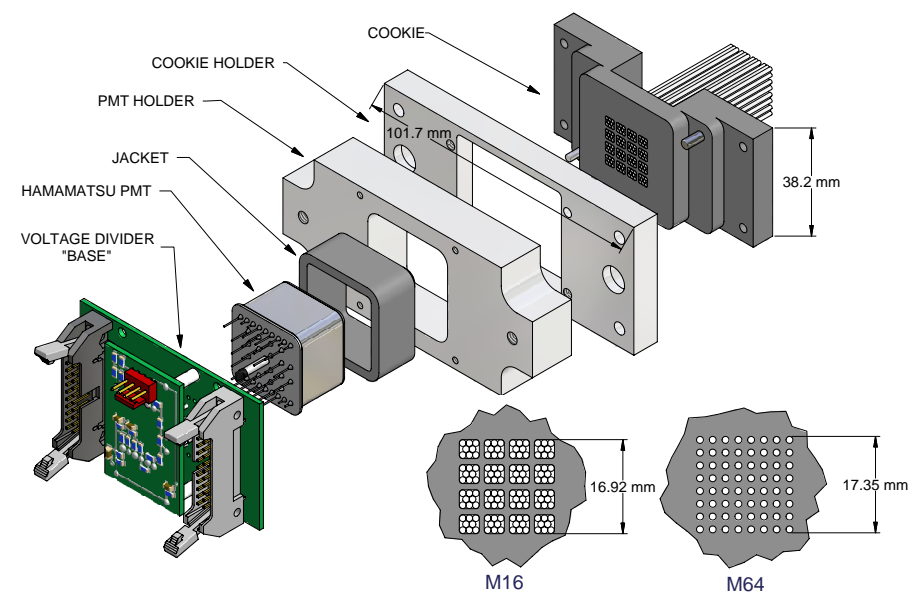

Figure 6.5: The M16 PMT mounting assembly. 8 fibers are placed on each M16 pixel. The Near Detector M64 mounting assembly is identical except that only one fiber is placed on each pixel. The fiber "cookie" layouts used in the two cases are shown face-on in the lower right of the figure.

layouts of M16 and M64 PMTs. One fiber is placed on each M64 PMT pixel, therefore each strip in the Near Detector is read out by one PMT pixel. To reduce the large instrumentation load at the Far Detector, a multiplexing technique (optical summing) 
is employed so that 8 fibers are connected to each M16 PMT pixel. Thus 8 strips from each plane are read out by the same PMT pixel. To help to determine which strip was actually hit, the 8 strips read out by a single pixel on one side of the detector are read out by 8 different pixels on the other side and the pattern of multiplexing has the maximum spacing possible between strips that are read out by the same PMT pixel.

The pixels in the Hamamatsu PMTs are known to suffer from crosstalk; about $7 \%$ of the signal from light on a given pixel may appear in neighboring pixels. The crosstalk phenomenon is an inherent property for multi-anode PMTs. Detailed studies indicate the PMT crosstalk effects are due to [143]:

1. Optical reflections and refractions: This effect is due to spread of photons emerging from the end of a fiber.

2. Imperfect focusing on the first dynode: The focusing grid between a photocathode and the first dynode is known to result in an imperfect distribution of photoelectrons. Photoelectrons, particularly those close to the edge of a pixel, can strike a neighboring dynode chain.

3. Charge spill-over: Due to an imperfect dynode focusing, some charge in the multiplication process may spread into the neighboring channels.

4. Capacitive coupling: There is a capacitive coupling between all the anodes.

We divide the crosstalk effects into two main categories. The optical crosstalk is comprised of the first two types itemized above and is characterized by an excess of single photoelectrons. The electrical crosstalk (charge crosstalk) includes the last two crosstalk types and is characterized by a shift in the pedestal position.

The crosstalk effects may lead to some confusion when we try to measure the energy deposited in showers, since neighboring strips are likely to be illuminated 
simultaneously. The problem can be minimized by reading out adjacent strips not with adjacent pixels, but with alternate pixels. Fig.6.6 shows two examples of pixel to

M64 PMT, Alner Box Type 1

\begin{tabular}{|c|c|c|c|c|c|c|c|}
\hline & & & & & & & \\
\hline 23 & 54 & 41 & 11 & 35 & 22 & 53 & 40 \\
\hline & 9 & 3 & 24 & & 42 & & \\
\hline 3 & 9 & 31 & 24 & 55 & 42 & 10 & 36 \\
\hline 39 & $\begin{array}{l}\text { Pixel } 17 \\
2\end{array}$ & 57 & 44 & 8 & 38 & 25 & 56 \\
\hline & & |xel|2 & & रा12 & & & truter \\
\hline 59 & 46 & 6 & 19 & 27 & 58 & 45 & 7 \\
\hline ixel 3 & $\begin{array}{l}P i x \mid 133 \\
3\end{array}$ & Pixel 33 & Pixel $3^{3}$ & Pixel 3 & Pixile & & Pixel 39 \\
\hline 4 & 17 & 29 & 60 & 47 & 5 & 18 & 28 \\
\hline 31 & $\begin{array}{l}\text { Pixel } 41 \\
62\end{array}$ & 49 & 3 & 16 & 30 & 61 & 48 \\
\hline $\begin{array}{l}\text { ixel } 4 \\
51\end{array}$ & $\begin{array}{l}\text { Pixel } 49 \\
1\end{array}$ & $\begin{array}{l}\text { Pixel } 5 \\
14\end{array}$ & $\begin{array}{l}\text { Pixel } 5 \\
32\end{array}$ & $\begin{array}{r}\text { Pixel 5 } \\
63\end{array}$ & 5 & 2 & 15 \\
\hline & & & & & & & \\
\hline 12 & 34 & 21 & 52 & 0 & 13 & 33 & 20 \\
\hline
\end{tabular}

(a) M64
M16 PMT, one example

\begin{tabular}{|c|c|c|c|}
\hline Pixel 0 & Pixel 1 & $\overline{\text { Pixel } 2}$ & Pixel 3 \\
\hline \begin{tabular}{|lll}
50 & 4 & 166
\end{tabular} & $\begin{array}{lll}154 & 39 & 16\end{array}$ & $\begin{array}{lll}190 & 167 & 51\end{array}$ & $\begin{array}{lll}178 & 155 & 40\end{array}$ \\
\hline $27 \quad 96$ & $85 \quad 62$ & 97 & 13286 \\
\hline \begin{tabular}{|lll}
73 & 189 & 143
\end{tabular} & $\begin{array}{lll}131 & 177 & 108\end{array}$ & $\begin{array}{lll}74 & 120 & 28\end{array}$ & \begin{tabular}{|lll}
63 & 17 & 109
\end{tabular} \\
\hline Pixel 4 & Pixel 5 & Pixel 6 & Pixel 7 \\
\hline $\begin{array}{lll}156 & 87 & 41\end{array}$ & $\begin{array}{lll}121 & 144 & 52\end{array}$ & $\begin{array}{lll}157 & 65 & 134\end{array}$ & \begin{tabular}{|lll}
53 & 145 & 30
\end{tabular} \\
\hline $\begin{array}{ll}133 & 18\end{array}$ & 19198 & $180 \quad 42$ & $\begin{array}{ll}7 & 122\end{array}$ \\
\hline $\begin{array}{llll}64 & 110 & 179\end{array}$ & \begin{tabular}{|lll}
29 & 75 & 6
\end{tabular} & $\begin{array}{lll}111 & 88 & 19\end{array}$ & $\begin{array}{lll}168 & 76 & 99\end{array}$ \\
\hline Pixel 8 & $\overline{\text { Pixel } 9}$ & $\begin{array}{l}\text { Pixel } 10 \\
\end{array}$ & $\begin{array}{l}\text { Pixel } 11 \\
\end{array}$ \\
\hline $\begin{array}{lll}8 & 146 & 31\end{array}$ & \begin{tabular}{|lll}
66 & 20 & 89
\end{tabular} & $\begin{array}{lll}101 & 55 & 32\end{array}$ & $\begin{array}{lll}67 & 136 & 21\end{array}$ \\
\hline $54 \quad 100$ & 112135 & $\begin{array}{ll}78 & 9\end{array}$ & $182 \quad 44$ \\
\hline \begin{tabular}{|lll}
77 & 123 & 169
\end{tabular} & $\begin{array}{lll}158 & 181 & 43\end{array}$ & $\begin{array}{lll}147 & 124 & 170\end{array}$ & $\begin{array}{lll}90 & 159 & 113\end{array}$ \\
\hline Pixel 12 & Pixel 13 & Pixel 14 & Pixel 15 \\
\hline \begin{tabular}{|lll}
22 & 91 & 137
\end{tabular} & \begin{tabular}{|lll}
33 & 10 & 56
\end{tabular} & $\begin{array}{lll}46 & 115 & 69\end{array}$ & $\begin{array}{lll}126 & 57 & 172\end{array}$ \\
\hline $\begin{array}{ll}68 & 160\end{array}$ & 10279 & $184 \quad 138$ & 14980 \\
\hline \begin{tabular}{|lll}
45 & 114 & 183
\end{tabular} & $\begin{array}{lll}125 & 148 & 171\end{array}$ & \begin{tabular}{|lll}
23 & 92 & 161
\end{tabular} & $\begin{array}{lll}34 & 11 & 103\end{array}$ \\
\hline
\end{tabular}

(b) M16

Figure 6.6: The mappings of plane strip number in the M64 PMT pixels (left) and M16 PMT pixels (right). The former serves one ND partial U plane and the latter servers $2 / 3$ of one FD plane.

plane strip mapping, one for the M64 PMT and one for the M16 PMT. Each M64 pixel is connected to one ND strip while each M16 pixel is connected to 8 FD strips (which are referred to as spots 1 through 8). Under this mapping scheme, any set of up to 10 adjacent strips that are simultaneously illuminated are read out by non-adjacent pixels. There is one difference between the M16 and M64 mappings. Fig.6.6 shows that the strips mapped to the diagonal neighboring pixels are generally 1,2 , and 3 strips away from the illuminated strip for the M16 PMT, which is not true for the M64 PMT. Even though the crosstalk between diagonal neighbors ("diagonal crosstalk") is much smaller than the crosstalk between adjacent neighbors ("adjacent crosstalk"), the FD diagonal crosstalk hits are more likely to be included in the reconstructed event since they are closer to the illuminated strips.

We now illustrate the pixel to strip mapping scheme with two cosmic ray events. 
Fig.6.7 shows one cosmic ray event in each of the two detectors. The dark hits
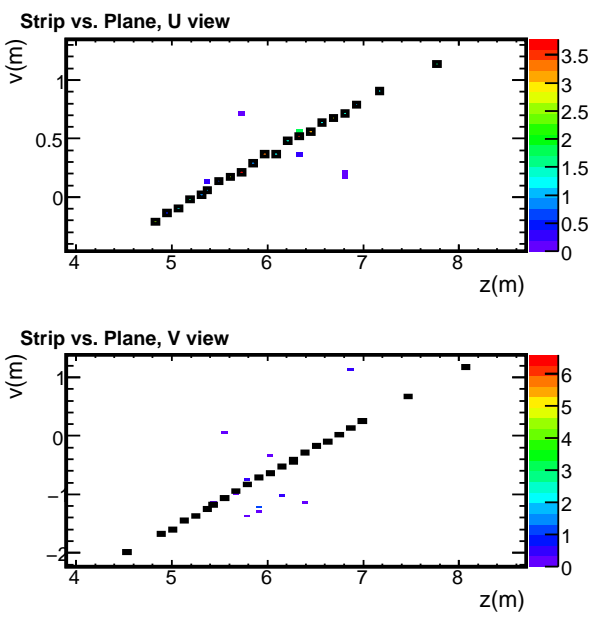

(a) One ND cosmic ray event
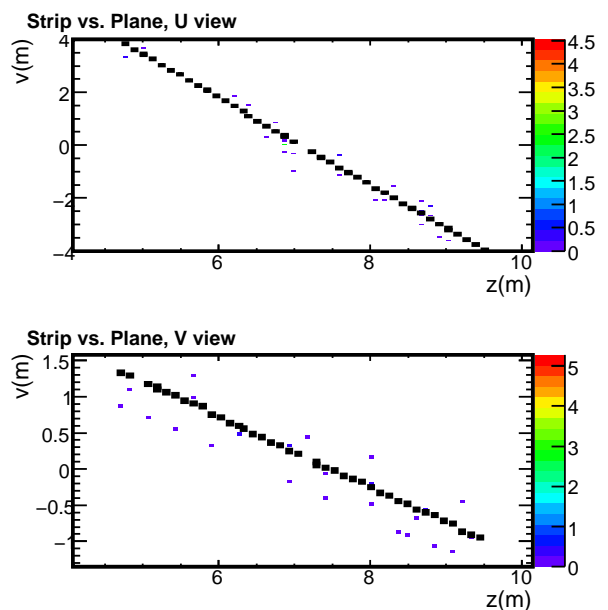

(b) One FD cosmic ray event

Figure 6.7: Two examples of cosmic ray events: (a) ND and (b) FD. The black boxes represent the track hits while the blue/purple hits around the track are crosstalk hits. The strip pulse-height is plotted in MIPs.

represent the muon track and the scattered hits around the track are consistent with the crosstalk hits. The crosstalk hits will generally be on the pixels neighboring the one associated with the track hit but they will be assigned to strips that are several strips away as a result of the pixel to strip mapping discussed above.

The cosmic ray muons are ideal tools for studying the PMT crosstalk. The cosmic ray muon usually hits only one strip on each plane and other hits on the same plane are mostly likely the crosstalk hits created by the illuminated strip. By measuring the charge of the track hit and the crosstalk hits, one can quantify crosstalk by calculating the fraction of charge that leaks into the neighboring pixels.

$$
X_{j, i}=\frac{Q_{\text {non-injected }(j)}}{Q_{\text {injected }(i)}}
$$

Since there are a large amount of cosmic ray events recorded at both detectors, one can measure such a quantity with very high accuracy. This approach was first proposed 
by Patricia Vahle of College of William \& Mary.

Before we discuss how we measure the magnitude of crosstalk, we will first briefly describe how the crosstalk is simulated in the Monte Carlo. Suppose there is a physics signal with charge $Q$ (in photoelectrons) hitting pixel $i$ on one PMT. We calculate the mean overall crosstalk charge as $Q_{x t a l k}=Q \times \sum_{j} X_{j, i}$, where $X_{j, i}$ is defined in Eq.(6.1), $i$ denotes the injected pixel, $j$ denotes the non-injected pixel, and the sum runs over all the non-injected pixels. We obtain the charge for optical crosstalk and electrical crosstalk separately based on the measured fractions. We then sample the total number of photoelectrons for the optical crosstalk with the assumption that the charge of optical crosstalk follows the Poisson distribution with the mean of $Q_{x t a l k}^{\text {optical }}$. Each photoelectron is then randomly assigned to one non-injected pixel on the same PMT according to the probabilities $X_{j, i} / \sum_{j} X_{j, i}$. The amount of optical crosstalk charge is then subtracted from the injected charge (charge conservation). The simulation of the electrical crosstalk is slightly different. Since the magnitude of electrical crosstalk is generally much smaller than the magnitude of optical crosstalk, we do not discuss the details of electrical crosstalk simulation here.

In the rest of this section, we will first describe our cosmic ray event selection, then move on to discuss the data and $\mathrm{MC}$ comparisons for the crosstalk hits and the attempt to improve the crosstalk simulation.

The cosmic ray muons can go in a wide range of directions. Fig.6.8 shows the cosmic ray muon track zenith angle and azimuth angle distributions. $\theta=0^{\circ}$ and $\theta=90^{\circ}$ correspond to the muons that go vertically and horizontally, respectively. $\phi \simeq 70^{\circ}$ and $\phi \simeq 250^{\circ}$ correspond to the muons that go parallel to the detector planes. If a muon track goes too steep or parallel to the detector planes, it can hit multiple strips on a single plane. In this case, it is rather difficult to reconstruct and calibrate the muon track and it is impossible to match the crosstalk hits with the 

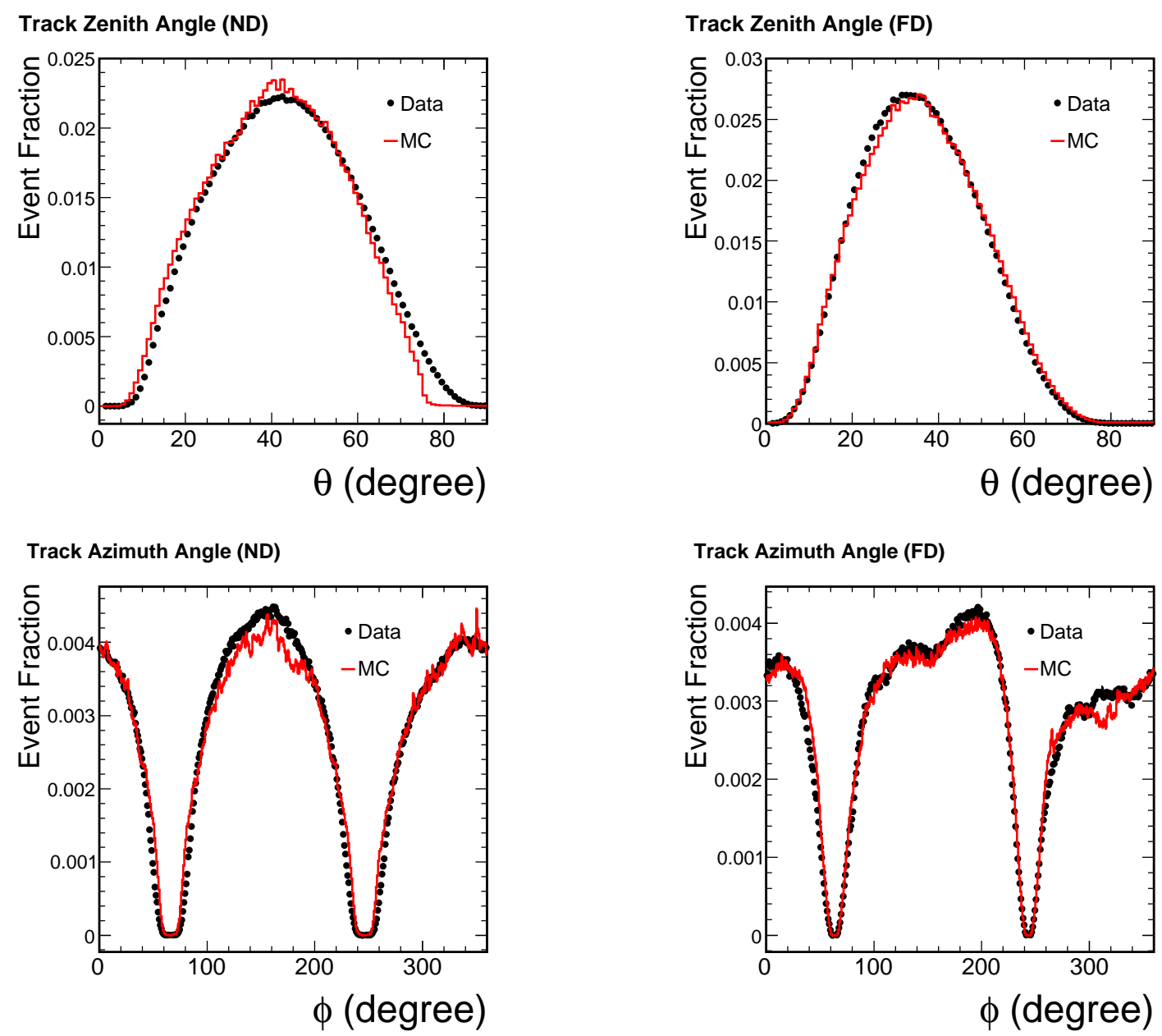

(a) ND

(b) FD

Figure 6.8: Cosmic ray muon zenith angle and azimuth angle distributions: (a) ND and (b) FD. 
muon hits. We make the following cuts on the zenith angle and azimuth angle to remove those events:

$$
30^{\circ}<\theta<90^{\circ}, \phi<10^{\circ} \text { or } 120^{\circ}<\phi<190^{\circ} \text { or } \phi>300^{\circ} \text {. }
$$

We require that there is one and only one track in the reconstructed cosmic ray event. Sometimes the muon can hit more than one strip on a single plane. In this case, it is almost impossible to determine which track hit is associated with the crosstalk hits on that plane. Therefore we ignore planes that have more than one track hit (but keep the other hits in the event). This would introduce a problem for the FD crosstalk measurement since the diagonal crosstalk tends to occur in the neighboring strips of the illuminated strip at the FD. Fortunately we can take advantage of the two stripends readout at FD. Each strip in the FD is read out by two PMTs independently, one PMT reading out one stripend on one side. A very important difference between real physics hits and crosstalk hits is that most crosstalk hits are single-ended (only read out at one strip end) while the physics hits are generally double-ended (except for small signals where readout from one end is below electronics threshold). This is because the physics hits are generated by photons transported along the strips in two directions while the crosstalk hits are induced at each end independently. Therefore, in the FD we only consider hits that are double-ended as track hits (physics hits). This helps us distinguish crosstalk hits from real physics hits and makes the measurement of diagonal crosstalk at FD possible. This trick does not apply to the ND where each strip is read out at only one end. But this is less of a problem for the ND since adjacent strips on one plane are read out by pixels that are well separated on the PMT face. Thus the crosstalk fractions between those pixels are negligible.

We want to use energetic muons so we select through going muons (instead of muons that stop inside the detectors) by requiring the track beginning and end are 
less than $20 \mathrm{~cm}$ away from the detector edge.

Occasionally the cosmic ray muon can create showers by knocking out delta ray electrons or through Bremsstrahlung radiation. These showers can obscure the muon hits thus makes it hard to match the crosstalk hits with the muon hits. Therefore we require that there is no reconstructed showers in the cosmic ray event.

Here is a summary of the cuts we applied:

- $30^{\circ}<\theta<90^{\circ}, \phi<10^{\circ}$ or $120^{\circ}<\phi<190^{\circ}$ or $\phi>300^{\circ}$

- number of tracks $=1$

- number of showers $=0$

- track beginning to detector edge $<20 \mathrm{~cm}$, track end to detector edge $<20 \mathrm{~cm}$ (through-going muons)

- only one hit associated with the track per plane

In our analysis, for each event that passes the cuts defined above, we take all the hits that are not associated with the reconstructed track as the crosstalk hits. We looked at three quantities of those crosstalk hits: pulse-height in PEs, relative transverse position w.r.t. the track hit (illuminated strip), and relative time w.r.t. the track hit. Fig.6.9 shows the ND crosstalk distributions for data and MC. The MC uses the default crosstalk fractions which were measured at the test stand. The left plot shows the strip pulse-height distributions. There is a big discrepancy between data and MC for hits below 1PE, which is consistent with what we saw in Fig.6.1. This seems to indicate that the mis-modeling of the low pulse-height hits can be explained by the mis-modeling of crosstalk hits. The middle plot shows the relative position w.r.t. the track. The four peaks at \pm 13 and \pm 20 strips correspond to the neighboring pixels of the illuminated pixel. They occur 13 or 20 strips away as 

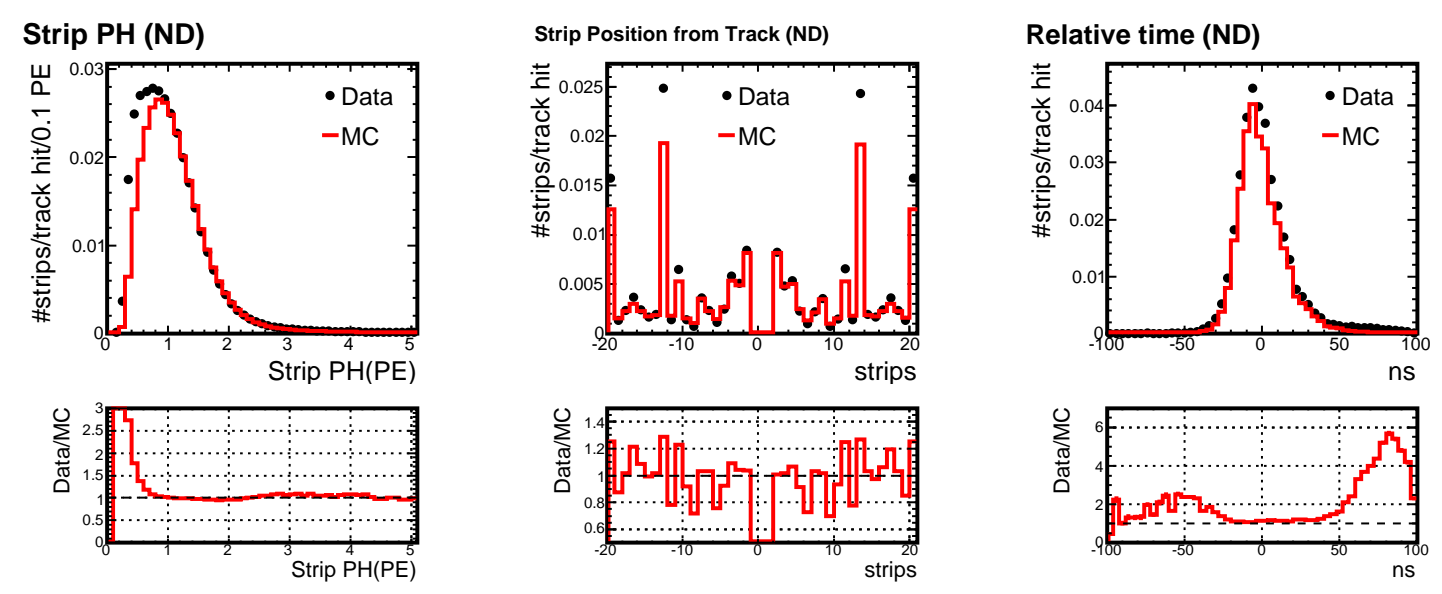

Figure 6.9: ND crosstalk distributions: strip pulse-height (left), relative position (middle), and relative time (right).

one expects according to the pixel to strip mapping shown in Fig.6.6(a), which is clear evidence that the hits we select are indeed crosstalk hits. The MC prediction underestimates data by roughly $30 \%$ at those positions, which indicates that the crosstalk was underestimated by $30 \%$ in the default M64 PMT simulation. The right plot shows the relative time of the crosstalk hits w.r.t. the track. Most of the hits occur within $50 \mathrm{~ns}$ of the muon hits. There is an excess of data hits between 50 ns and 100 ns. This excess may be caused by the PMT after-pulsing which is not currently modeled in the MC simulation. However, those hits only make up a very small fraction of the total hits.

Fig.6.10 shows the similar distributions for the Far Detector. Some unique characteristics of FD crosstalk pattern can be seen in those plots. The left plot shows the pulse-height distributions. The data distribution has two peaks, one at 1PE while the other at around $0.3 \mathrm{PE}$, which represent two components of crosstalk. The single PE peak corresponds to the optical crosstalk while the peak below 1PE corresponds to the electrical crosstalk. The optical crosstalk is reasonably well modeled by the MC 

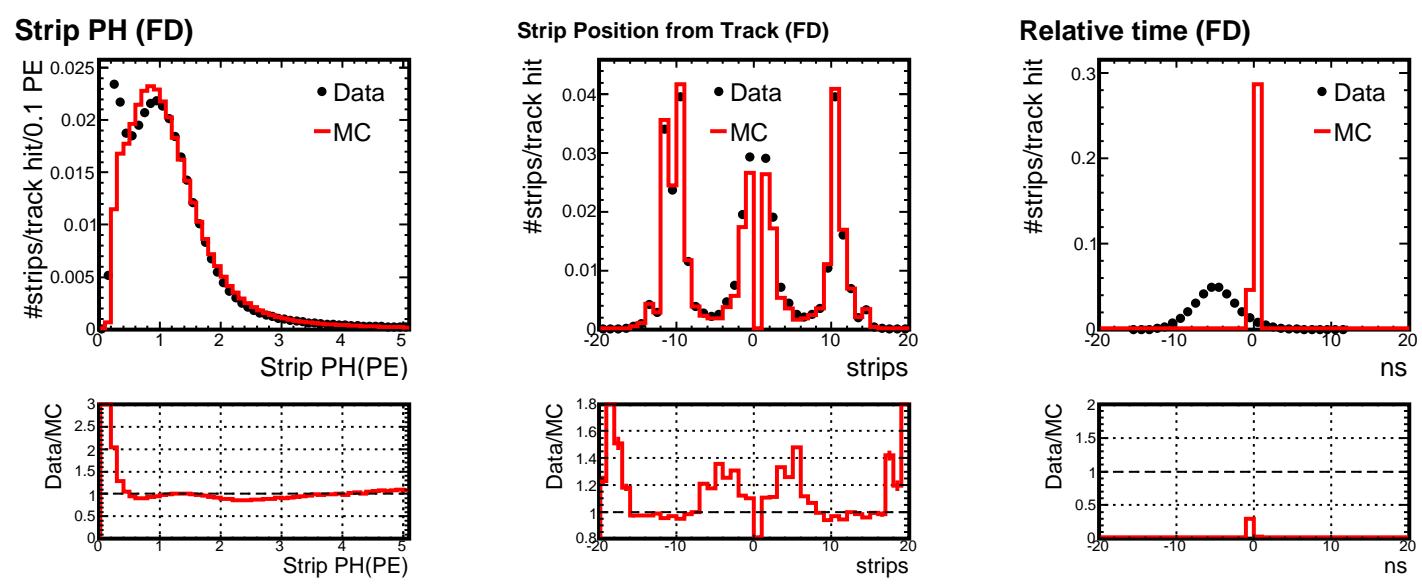

Figure 6.10: FD crosstalk distributions: strip pulse-height (left), relative position (middle), and relative time (right).

simulation while the electrical crosstalk is largely underestimated. The Near Detector electronics apply a $0.2 \mathrm{PE}$ sparsification threshold to all the digits, which highly suppresses the electrical crosstalk (Fig.6.9, left plot).

The middle plot of Fig.6.10 shows the relative strips position from the track. The two peaks at around \pm 10 strips are crosstalk hits in the adjacent pixels while the peak at $\pm 1, \pm 2$, and \pm 3 strips are crosstalk hits in the diagonal neighboring pixels according to Fig.6.6(b). The adjacent crosstalk is slightly overestimated while the diagonal crosstalk is underestimated.

The right plot of Fig.6.10 shows the relative time w.r.t. the track. The FD timing is based on the PMT dynode timing. Therefore the crosstalk times are identical to the track hit time, which is what the MC distribution indicates. The calibration software applies a correction for the time it takes a signal to reach the electronics threshold and be read out (smaller signals take longer to trigger). This delay is called "timewalk" and it is only applied to the data. This is the major source of the small data and $\mathrm{MC}$ difference shown in the right plot of Fig.6.10. Right now, no FD timing information is used in this analysis. 
The strip pulse-height and position distributions indicate that the crosstalk is not well modeled in the simulation. Our first attempt to improve the crosstalk simulation is to scale the crosstalk fraction in the MC. Fig.6.11 shows the ND crosstalk distri-
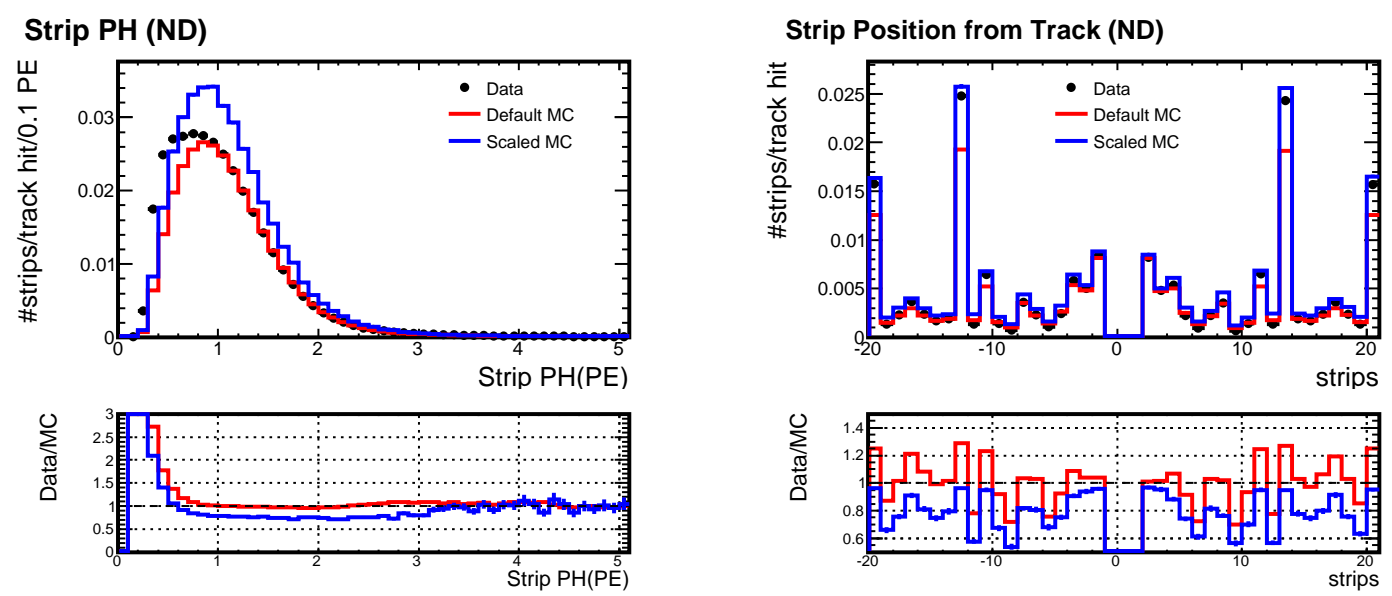

Figure 6.11: ND crosstalk distributions: strip pulse-height (left) and relative position (right). The red lines represent the default $\mathrm{MC}$ while the blue lines represent the modified MC where the crosstalk fraction is scaled up by $35 \%$.

butions after we scale the crosstalk fraction up by $35 \%$. It can be seen from the right plot that after scaling the $\mathrm{MC}$, the agreement between data and $\mathrm{MC}$ improves at \pm 11 , \pm 13 , and \pm 20 strips but deteriorates at other locations. The left plot shows the strip pulse-height distributions and the scaled MC apparently overshoots the data.

Fig.6.12 shows the FD crosstalk distributions after we scale the diagonal crosstalk fraction up by $40 \%$ and scale the other crosstalk down by $5 \%$. While the strip pulseheight distribution remains almost unchanged, the strip position distribution of the scaled MC agrees much better with the data.

Scaling up and down the crosstalk fractions looks promising but it does not solve all the problems. It does not improve the shape of the pulse-height distribution, nor does it treat the optical crosstalk and the electrical crosstalk separately. Moreover it does not completely take into account the pixel to pixel variations (even though the 

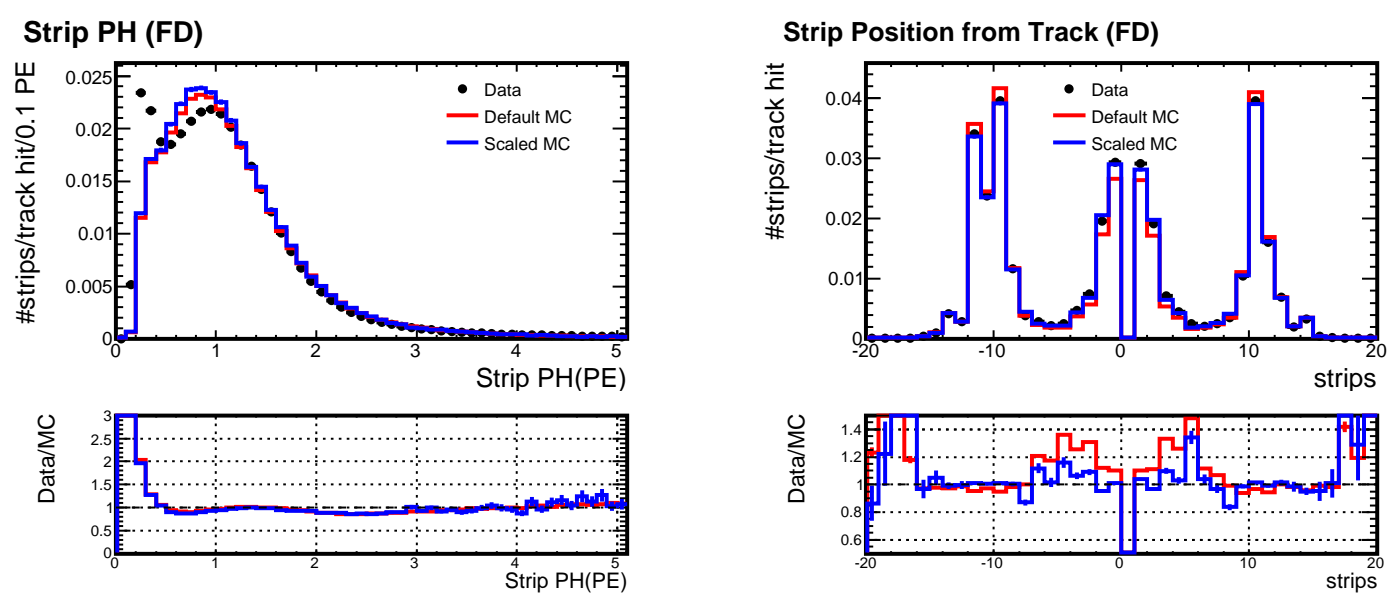

Figure 6.12: FD crosstalk distributions: strip pulse-height (left) and relative position (right). The red lines represent the default $\mathrm{MC}$ while the blue lines represent the modified MC where the diagonal crosstalk fraction is scaled up by $40 \%$ while the other crosstalk is scaled down by $5 \%$.

FD scaling scheme tried to address this issue). We developed a more sophisticated method to tune the crosstalk fractions. For each hit pixel (i.e. the pixel that maps to the strip that the muon passed through), we find the charge of the crosstalk hits on all other pixels on that PMT. For each crosstalked pixel we plot the crosstalk charge as a function of the injected charge (muon hit charge + all crosstalk charge). We then fit a straight line to that distribution and the slope is the fraction of charge that leaks from the hit pixel to the crosstalked pixel. To get the slope we average over all the PMTs, i.e. we believe that the crosstalk pattern is mainly determined by the relative locations of pixels and we ignore the variations between different PMTs. For the crosstalk hits, hits below $0.5 \mathrm{PE}$ are taken as electrical crosstalk while hits above $0.8 \mathrm{PE}$ are taken as optical crosstalk. We do not use hits between $0.5 \mathrm{PE}$ and $0.8 \mathrm{PE}$ since it is difficult to identify the source (electrical or optical crosstalk) of those hits. We do this for both data and $\mathrm{MC}$ so we can get a correction factor that scales the MC to look like the data.

We will first show the details of ND crosstalk tuning and then show the results 
of FD crosstalk tuning. Fig.6.13 shows the strip pulse-height of the muon hits read out by Pixel 36 of M64 PMT (middle plot) and the crosstalk generated by Pixel 36 (surrounding plots). We choose Pixel 36 because it is close to the center of the M64 PMT face. We only show the adjacent and diagonal neighboring pixels since the crosstalk in the faraway pixels is much smaller. The charge of muon hits is very well modeled by the MC. The charge of crosstalk hits is reasonably well modeled, but the MC simulation underestimates the crosstalk hits below $1 \mathrm{PE}$, which is consistent with what we showed in Fig.6.9.

Fig.6.14 shows the crosstalk charge as a function of the charge injected into Pixel 36 of M64 PMT. The slope of the distributions represents the fraction of charge that leaks into a specific pixel from Pixel 36. MC Simulation underestimates the crosstalk fractions for all the 8 pixels.

Fig.6.15 shows our attempt to correct the MC crosstalk simulation. We fit a straight line to the crosstalk charge as a function of injected charge. We do this for both data and MC. The ratio of the two slopes is the correction factor we should apply to the MC crosstalk simulation. In order to address the difference between optical crosstalk and electrical crosstalk, we assign hits whose pulse-heights are greater than 0.8 PE to be optical crosstalk and those whose pulse-heights are less than $0.5 \mathrm{PE}$ to be electrical crosstalk. Fig.6.15 indicates that the corrections to the optical crosstalk simulation are quite small while the corrections to the electrical crosstalk are quite large. This is consistent with the good agreement between data and $\mathrm{MC}$ above $1 \mathrm{PE}$ and large discrepancy below 1PE shown in Fig.6.9 (left plot). However, one thing we should note is that it is very difficult to distinguish between optical crosstalk and electrical crosstalk since hits below $0.5 \mathrm{PE}$ still have a large contribution from the optical crosstalk. We noticed that the charge of electrical crosstalk does not scale very well with the injected charge. This may be caused by the fact that we do not accept electrical crosstalk with pulse-height higher than $0.5 \mathrm{PE}$ thus the tail of the 

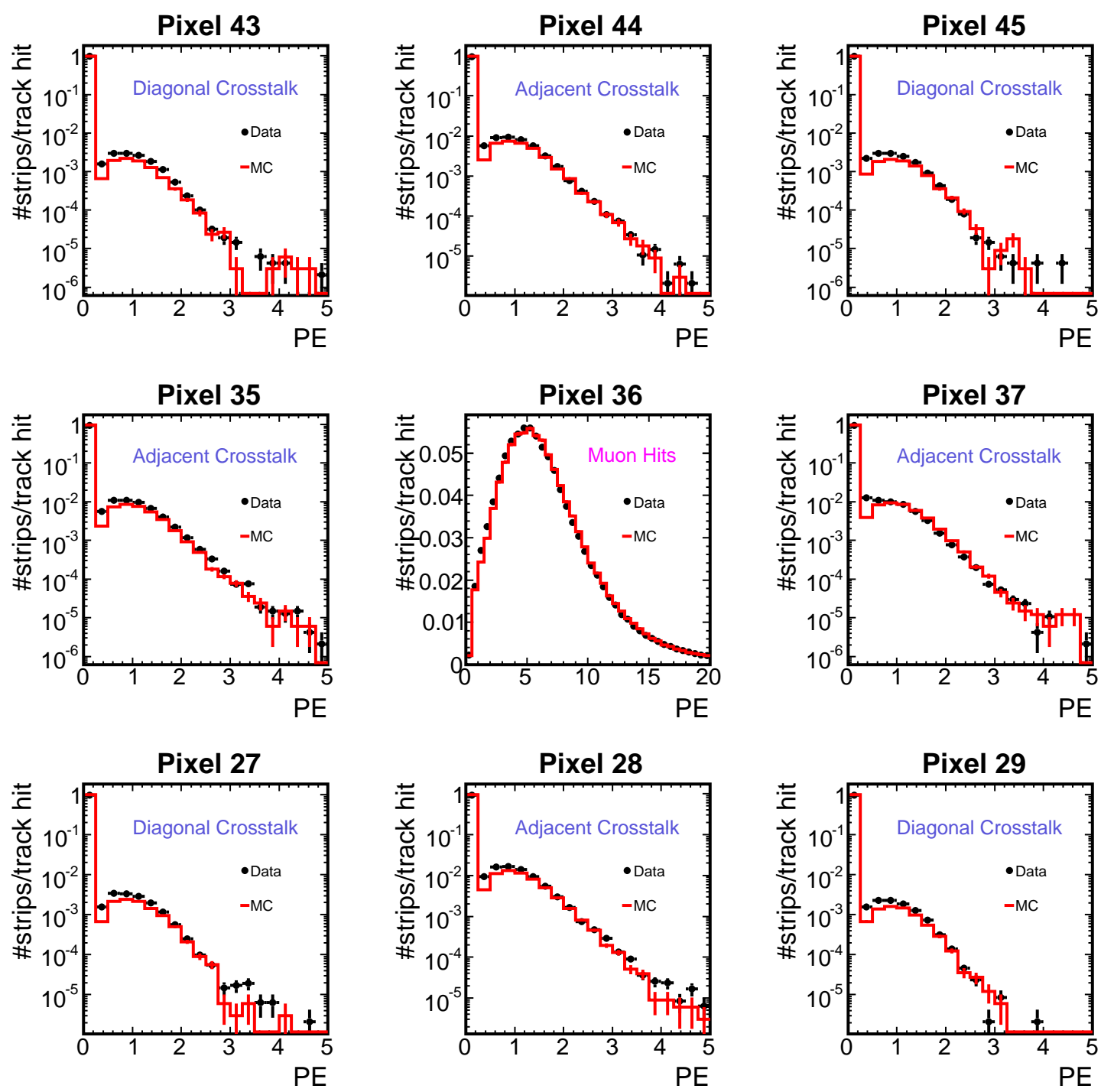

Figure 6.13: Strip pulse-height of the muon hits read out by Pixel 36 of M64 PMT (middle plot) and the crosstalk generated by Pixel 36 (surrounding plots). 

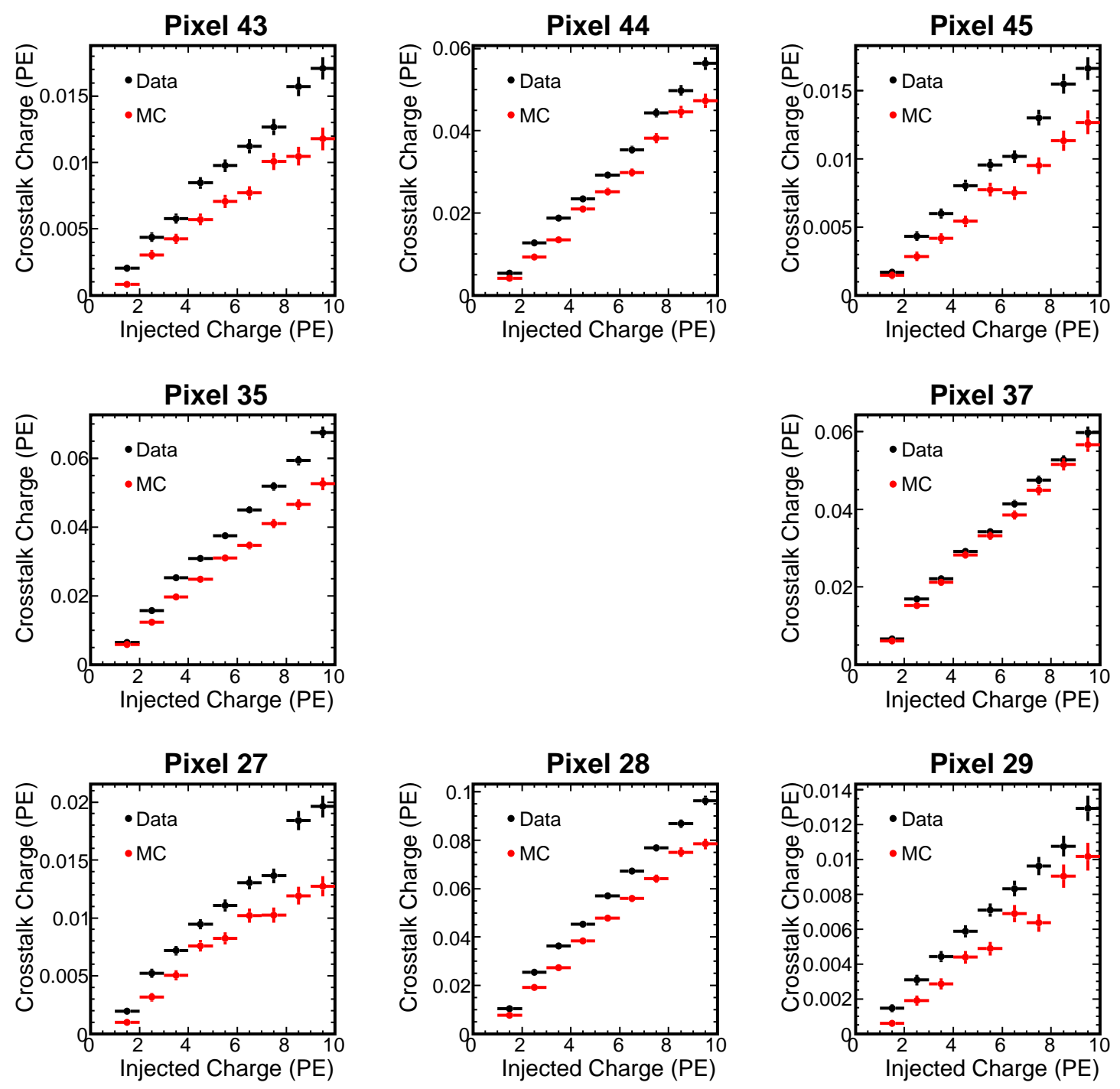

Figure 6.14: Crosstalk charge as a function of injected charge (injected pixel is Pixel $36)$. 
pulse-height distribution is truncated.

Fig.6.16 shows the ND crosstalk fractions obtained from data for the overall crosstalk of each of the 64 pixels (a), the optical adjacent crosstalk for each pixel (b), and the optical diagonal crosstalk for each pixel (c). It can be seen that the average ND overall crosstalk fraction is $4.7 \%$, the average optical adjacent crosstalk fraction is $0.7 \%$ per pixel, and the average optical diagonal crosstalk fraction is $0.16 \%$ per pixel.

Fig.6.17 shows the correction factors we need to apply to the ND MC simulation. The corrections for optical crosstalk are rather small and the average correction factor is 1.09 for adjacent crosstalk and 1.18 for diagonal crosstalk. The corrections for electrical crosstalk are quite large and the average correction factor is roughly 2.4 for both the adjacent crosstalk and the diagonal crosstalk.

We apply the same procedure to the FD data and MC. Fig.6.18 shows the distributions of the measured overall crosstalk fractions of each of the 128 spots of FD M16 PMTs (a), the optical adjacent crosstalk for each spot (b), and the optical diagonal crosstalk for each spot (c). The 128 entries in Fig.6.18 (a) correspond to 16 pixels times 8 spots per pixel. It can be seen that the average FD overall crosstalk fraction is $6.9 \%$, the average optical adjacent crosstalk fraction is $1.0 \%$ per spot, and the average optical diagonal crosstalk fraction is $0.46 \%$ per spot. The average FD crosstalk fraction is generally higher than the average ND crosstalk fraction. Fig.6.18 (b) and (c) indicate that the FD crosstalk fractions are more spread out than the ND crosstalk fractions. This is caused by the fact that there are 8 strips connected to one M16 pixel and different pixels induce different amounts of crosstalk charge depending on the specific location of each spot on the pixel. In Fig.6.19 we show the location on PMT Pixel 5 of the 8 spots (referred to below as spots 1 to 8 ) which are illuminated by the fibers of the 8 strips coupled to that pixel. A signal hitting spot 1 induces a lot more crosstalk charge in pixels 1 and 4 than in pixels 6 and 9 while a signal hitting 

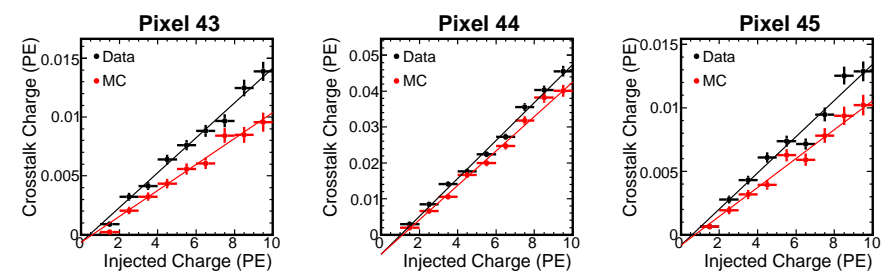

Pixel 35

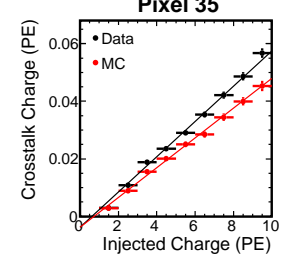

Pixel 37
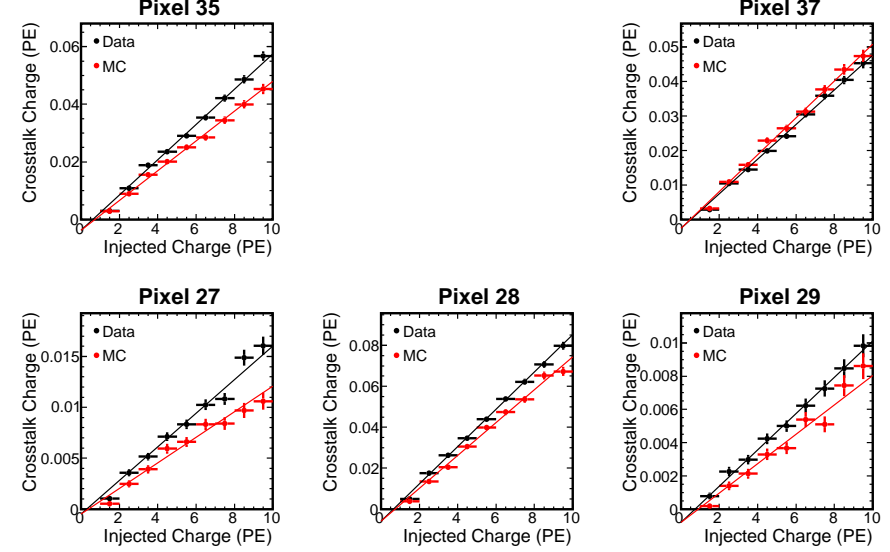

(a) Optical Crosstalk
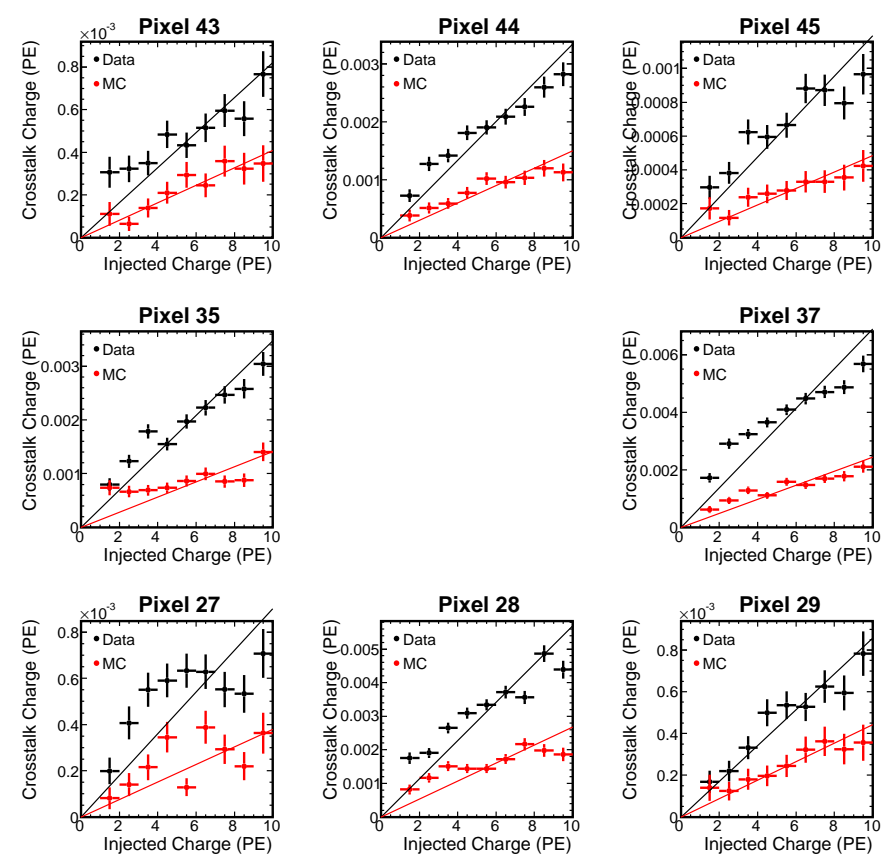

(b) Electrical Crosstalk

Figure 6.15: Fitted crosstalk charge as a function of injected charge for Pixel 36 in the adjacent and diagonal neighbors. Hits whose pulse-heights are greater than 0.8 $\mathrm{PE}$ are taken as optical crosstalk (a) while those whose pulse-heights are less than 0.5 PE are taken as electrical crosstalk (b). To get the correct mean of the crosstalk charge, zero charge hits are included in both samples. 

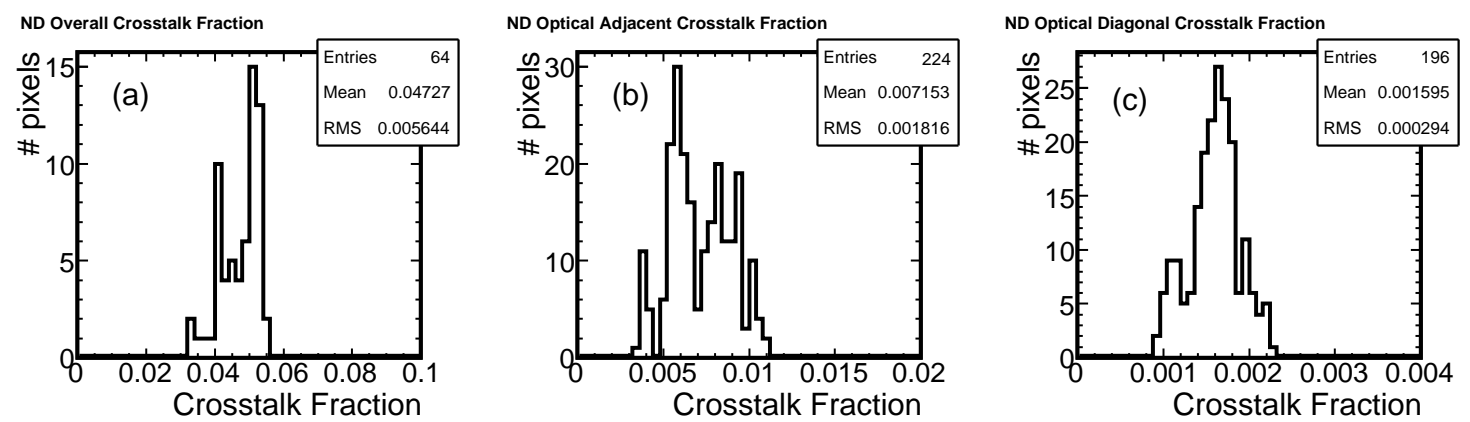

Figure 6.16: Measured crosstalk magnitude for ND M64 PMT pixels: (a) overall crosstalk for each of the 64 pixels, (b) optical adjacent crosstalk for each pixel, and (c) optical diagonal crosstalk for each pixel.
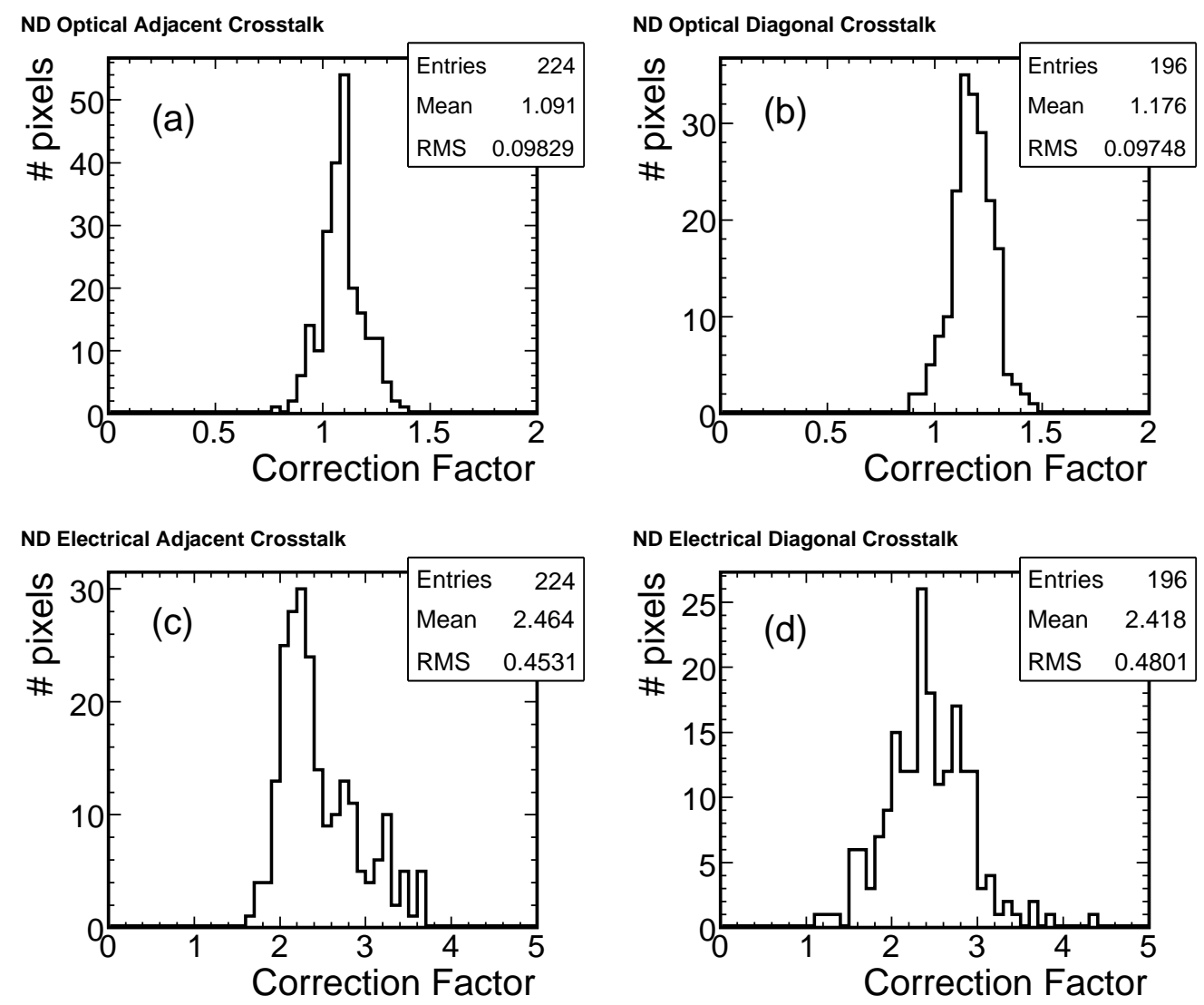

Figure 6.17: The correction factors we need to apply to the ND MC simulation: (a) optical adjacent crosstalk, (b) optical diagonal crosstalk, (c) electrical adjacent crosstalk, and (d) electrical diagonal crosstalk. 

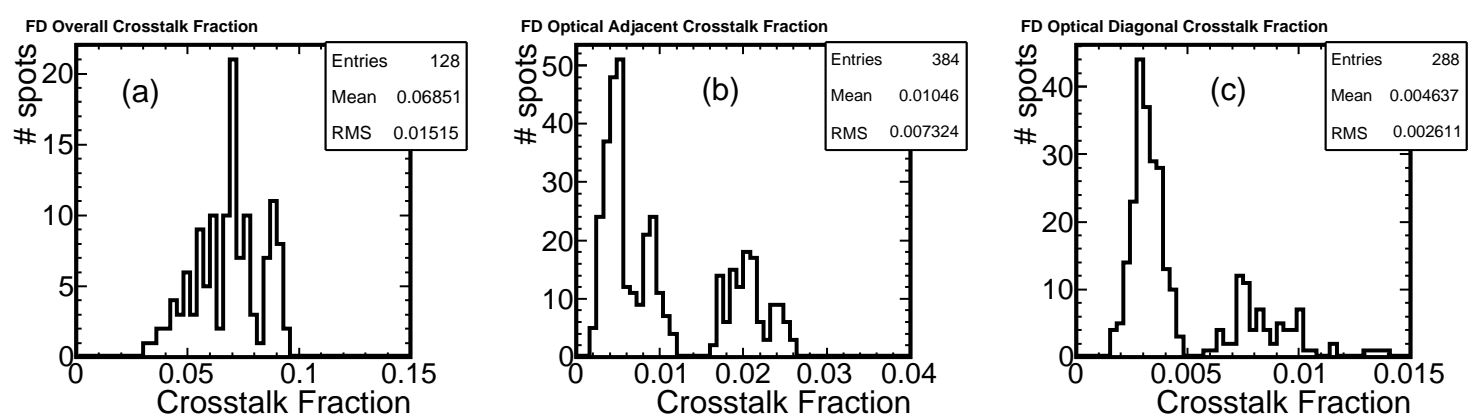

Figure 6.18: Measured crosstalk magnitude for FD M16 PMT spots: (a) overall crosstalk for each of the 128 spots, (b) optical adjacent crosstalk for each spot, and (c) optical diagonal crosstalk for each spot.

spot 8 induce a lot more crosstalk charge in pixels 6 and 9 than in pixels 1 and 4 .

\begin{tabular}{|c|c|c|}
\hline Pixel 0 & Pixel 1 & Pixel 2 \\
\hline Pixel 4 & $\begin{array}{l}(1) 3 \\
4(5) \\
6(7) 8\end{array}$ & Pixel 6 \\
\hline Pixel 8 & Pixel 9 & Pixel 10 \\
\hline
\end{tabular}

Figure 6.19: The position of Pixel 5 of the 8 spots corresponding to the location of the 8 fibers coupled to that pixel.

Fig.6.20 shows the correction factors that we need to apply to the FD MC simulation on a spot by spot basis. The corrections for optical crosstalk are rather small and the average correction factor is 0.9 for the adjacent crosstalk and 1.1 for the diagonal crosstalk. The corrections for electrical crosstalk are quite large and the 

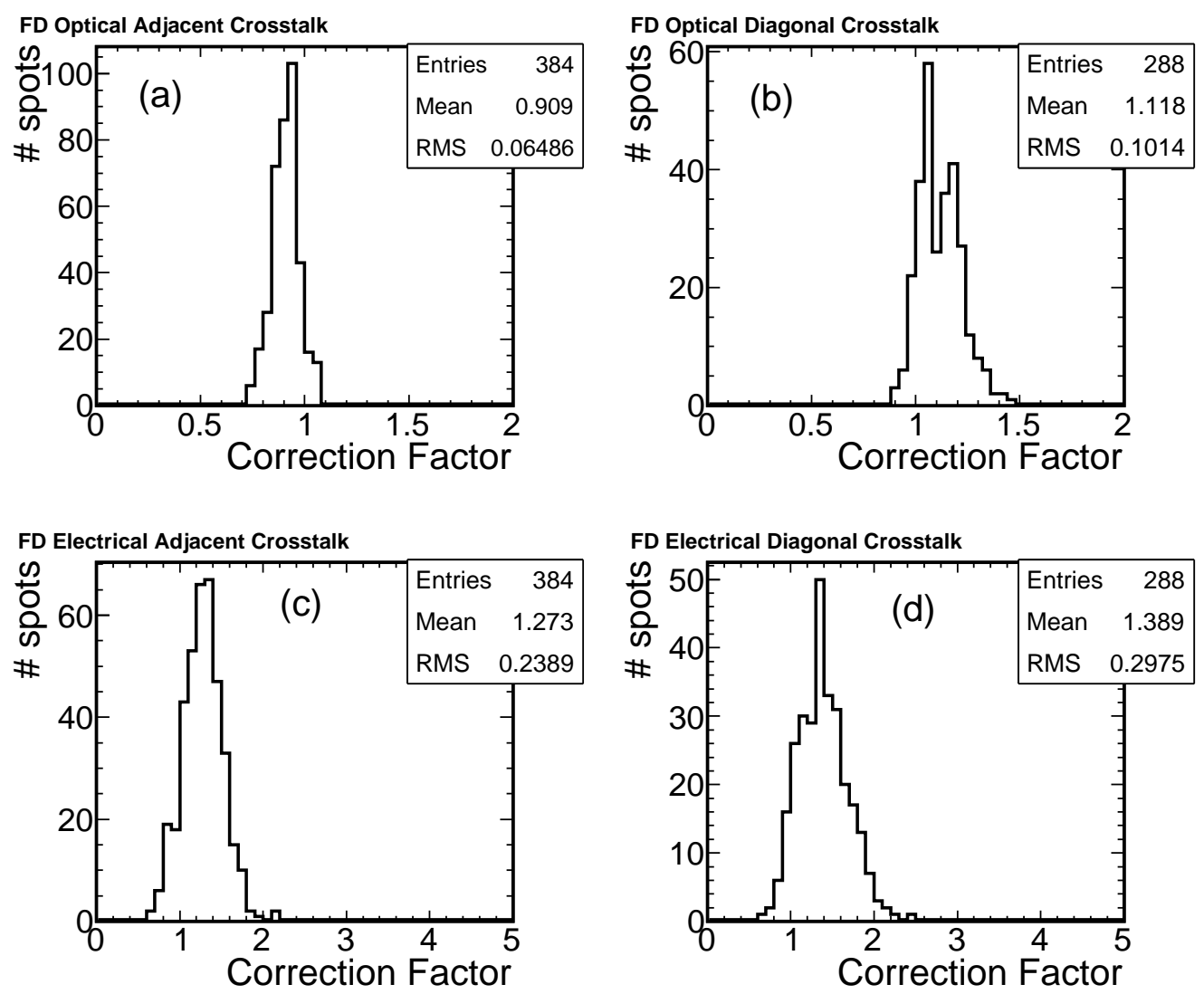

Figure 6.20: The correction factors we need to apply to the FD MC simulation: (a) optical adjacent crosstalk, (b) optical diagonal crosstalk, (c) electrical adjacent crosstalk, and (d) electrical diagonal crosstalk. 
average correction factor is roughly 1.3 for both the adjacent crosstalk and the diagonal crosstalk. These correction fractions agree qualitively with the scale factors we applied in Fig.6.12 (on average we need to scale up optical diagonal crosstalk and scale down optical adjacent crosstalk).
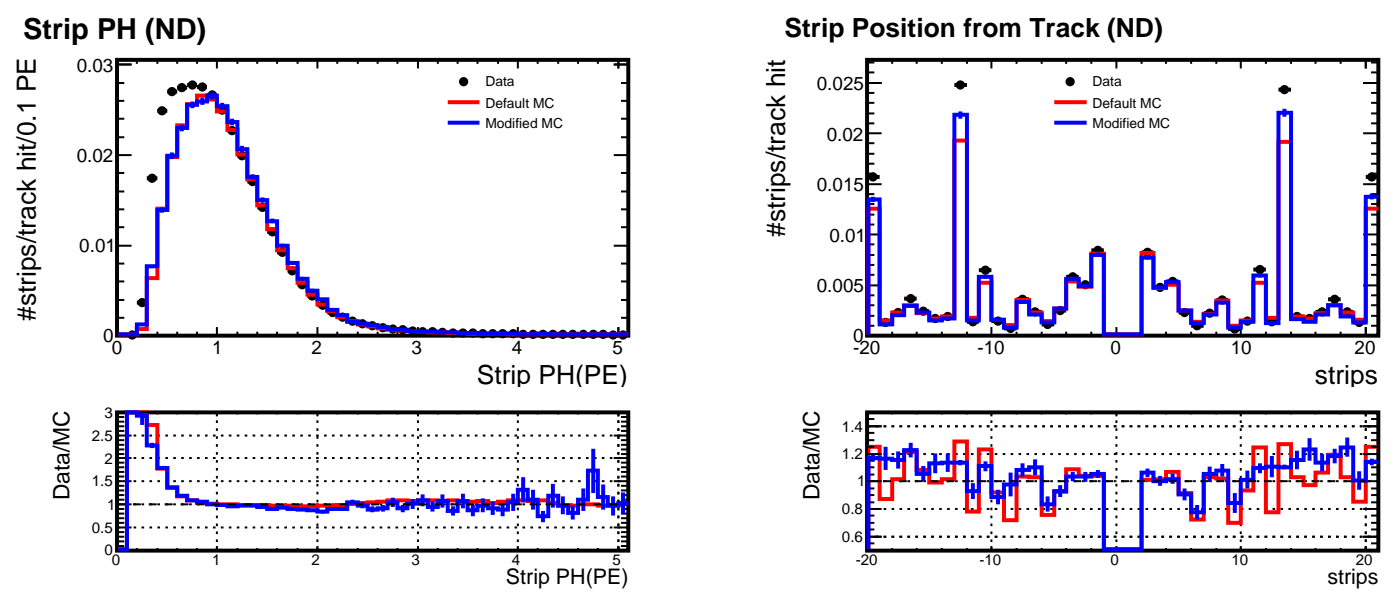

Figure 6.21: ND crosstalk distributions: strip pulse-height (left) and relative position (right). The red lines represent the default MC while the blue lines represent the MC with updated crosstalk fractions.

In order to validate the tuning of the crosstalk fractions, we generated a small sample of $\mathrm{MC}$ with the new crosstalk fractions. We multiply the default crosstalk fractions by the correction factors we measured above to obtain the new crosstalk fractions and use them for the simulation of crosstalk. The crosstalk distributions for the new MC are shown in blue in Fig.6.21 and Fig.6.22. The ND strip pulse-height distribution does not change much with the updated crosstalk. The ND strip position distribution is improved with the updated crosstalk. But the data and MC agreement is not perfect. The FD strip pulse-height distribution is greatly improved below 1 $\mathrm{PE}$ with the updated crosstalk. The MC simulation now predicts a second peak at around $0.3 \mathrm{PE}$ as a result of increased electrical crosstalk. But there is still a rather large discrepancy between data and MC for hits below 0.4 PE. The FD strip position 

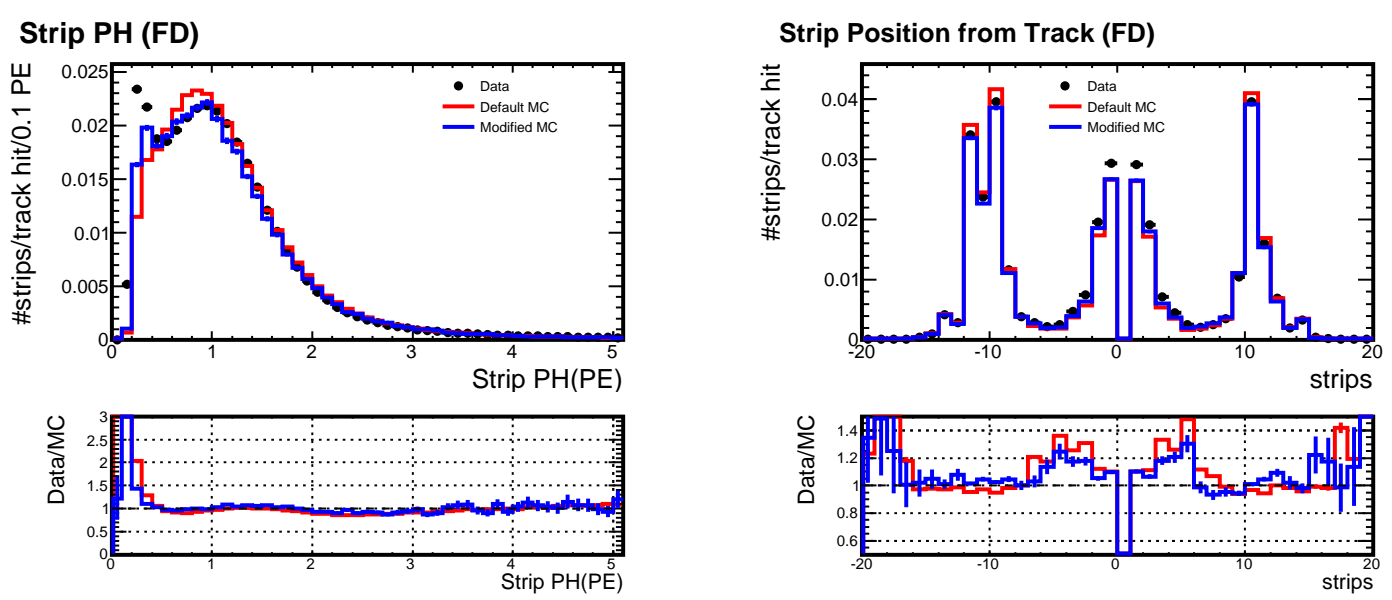

Figure 6.22: FD crosstalk distributions: strip pulse-height (left) and relative position (right). The red lines represent the default $\mathrm{MC}$ while the blue lines represent the $\mathrm{MC}$ with updated crosstalk fractions.

distribution is also improved with the updated crosstalk.

We have demonstrated the tuning of crosstalk fractions using cosmic data can improve the crosstalk simulation. However the cosmic data and the spill (neutrino) data are taken under different trigger conditions. The cosmic trigger is based on the clustering of hits in the detector (primarily requiring at least 4 planes out of any group of 5 contiguous planes in the detector contain one or more hits) to gather outof-spill events while the spill trigger is based on spill times to gather beam events. The cosmic trigger window is 190ns(pre-trigger window)+190ns(trigger window) for the ND and $30 \mu$ s(pre-trigger window)+190ns(trigger window) for the FD while the spill trigger window is $19 \mu \mathrm{s}$ (1000 buckets). Also the angular distributions of cosmic muons and muons from neutrino interactions are different. However the crosstalk measurements we made using the cosmic data and MC should not be sensitive to those differences. Therefore the correction factors we obtained can be applied to the spill event simulation. 


\subsection{Changes to the $\nu_{e}$ analysis}

In the previous section, we demonstrated that the excess of low pulse-height hits in the data is consistent with being crosstalk hits. We also described our attempt to improve the crosstalk simulation. The $\mathrm{MC}$ with the updated crosstalk fractions agrees better with the data. However the MC simulation still underestimates hits below $1 \mathrm{PE}$ especially for the ND. There may be other effects that account for the mis-modeling of low pulse-height hits. Those effects may include incorrectly modeled single PE width, gains, and threshold effect. We certainly do not want to overtune crosstalk to compensate for other effects.

The $\nu_{e}$ group has invested efforts in making the event selection less sensitive to the low pulse-height hits. Eventually we decided to make two major changes to the $\nu_{e}$ analysis:

1. Cut out low pulse-height hits at analysis time. We only use strips whose pulseheights are greater than $2 \mathrm{PE}$ to compute the discriminative variables. The advantage of this approach as opposed to redoing the reconstruction as we showed in the second section is that it is a lot faster and we do not need to validate the changes to the reconstructions.

2. Refine PID variables. The original $\nu_{e}$ artificial neural network (ANN) had 30 variables and proved to be sensitive to the low pulse-height hits. We removed some variables that are very sensitive to the low pulse-height hits and hard to fix at analysis time. We modified several variables to eliminate/reduce their sensitivity to the low pulse-height hits. The changes include ignoring hits that are more than 9 strips away from the event vertex transversely and weighting each strip by pulse-height squared instead of pulse-height to downgrade the importance of the low pulse-height hits. We constructed a new neural network with 11 modified variables. The details of this neural network were discussed 
in Chapter 4.

We decided to use the MC generated with the older crosstalk fractions for the $\nu_{e}$ analysis since the new ANN PID is largely insensitive to the crosstalk hits. In order to evaluate the systematic error resulting from the uncertainties in the crosstalk modeling, we generated a small sample of $\mathrm{MC}$ with the newly measured crosstalk fractions. We compared the results of the two MC samples and we take the difference as the systematic error from the crosstalk modeling. Fig.6.23 shows the reconstructed

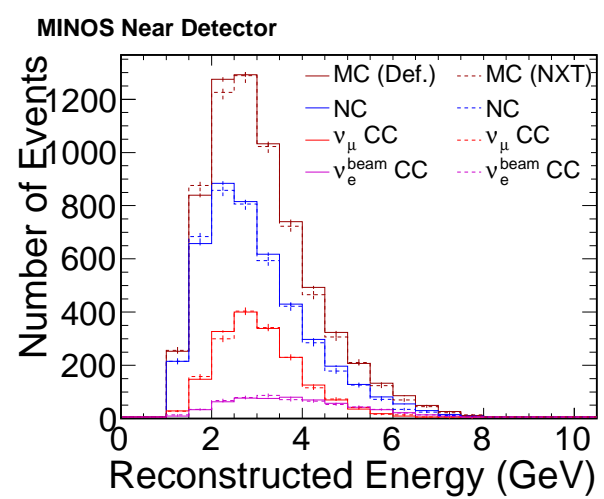

(a) ND

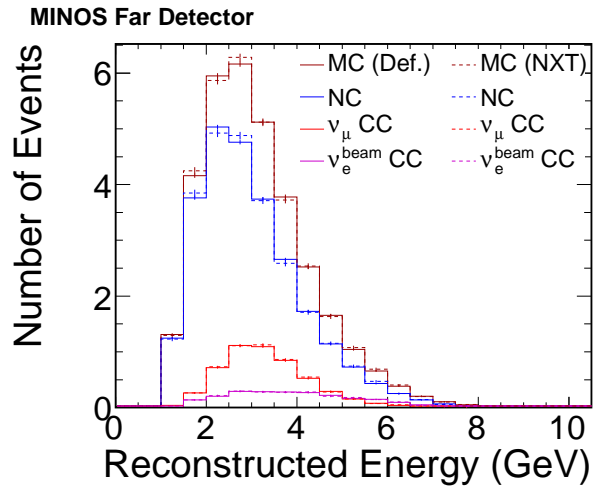

(b) FD

Figure 6.23: Energy spectra of background events accepted by the new ANN PID as $\nu_{e}$ candidates. (a) ND (b) FD. The solid lines represent $\mathrm{MC}$ with the older crosstalk fractions (Def) while the dashed lines represent $\mathrm{MC}$ with the new crosstalk fractions (NXT). The contribution of $\nu_{\tau}$ events is not included in the FD spectra. The oscillation parameters are $\left|\Delta m_{31}^{2}\right|=2.4 \times 10^{-3} \mathrm{eV}^{2}, \sin ^{2} 2 \theta_{13}=0.15, \sin ^{2} 2 \theta_{23}=1$. The spectra are normalized to FD POT $=3.25 \times 10^{20}$ (2-year exposure) and ND POT $=$ $1 \times 10^{19}$. The errors are only statistical errors.

energy spectra of background events accepted by ANN PID at both detectors. The solid lines represent $\mathrm{MC}$ with the older crosstalk fractions (Def) while the dashed lines represent $\mathrm{MC}$ with the new crosstalk fractions (NXT). Table 6.1 shows the numbers of accepted events at both detectors for MC with default crosstalk fractions (Def) and MC with the new crosstalk fractions (NXT). The two crosstalk models indeed predict very similar background rates which means the new ANN PID is rather 
insensitive to the crosstalk hits. With the new MC, the Far/Near ratio is shifted by $2.2 \% \pm 1.3 \%$ for the $\mathrm{NC}+\nu_{\mu} \mathrm{CC}$ background. We decided to use the older $\mathrm{MC}$ for the first MINOS $\nu_{e}$ analysis because of time constraints. Since the NC and $\nu_{\mu} \mathrm{CC}$ background components are predicted through Far/Near extrapolation, we will make corrections to those background predictions based on the differences showed in Table 6.1, i.e. we will scale up the $\mathrm{FD} \mathrm{NC}+\nu_{\mu} \mathrm{CC}$ background prediction by $2.2 \%$ and take the statistical error on that scale, namely $1.3 \%$, as the systematic error associated with crosstalk mismodeling. For the beam $\nu_{e}$ and $\nu_{\tau}$ background components, we do not make the corrections since their contributions to the total background are relatively small and the differences showed in Table 6.1 are negligible. We take the differences as the systematic errors. We do not correct our signal prediction since the efficiency of selecting genuine $\nu_{e}$ events is not sensitive to the crosstalk hits.

\begin{tabular}{c|c|cccc}
\hline \multicolumn{2}{l|}{} & $\mathrm{NC}$ & $\nu_{\mu} \mathrm{CC}$ & $\mathrm{NC}+\nu_{\mu} \mathrm{CC}$ & $\nu_{e}^{\text {beam }} \mathrm{CC}$ \\
\hline \multirow{4}{*}{ FD } & MC(Def.) & $25.71 \pm 0.09$ & $5.19 \pm 0.05$ & $30.90 \pm 0.10$ & $2.21 \pm 0.04$ \\
& $\mathrm{MC}(\mathrm{NXT})$ & $25.74 \pm 0.17$ & $5.26 \pm 0.06$ & $31.00 \pm 0.18$ & $2.22 \pm 0.05$ \\
& $\mathrm{NXT} /$ Def-1 & $0.1 \% \pm 0.7 \%$ & $1.4 \% \pm 1.5 \%$ & $0.3 \% \pm 0.7 \%$ & $0.5 \% \pm 2.9 \%$ \\
\hline \multirow{4}{*}{$\mathrm{ND}$} & $\mathrm{MC}$ (Def.) & $4429 \pm 17$ & $1742 \pm 10$ & $6171 \pm 20$ & $593 \pm 6$ \\
& $\mathrm{MC}(\mathrm{NXT})$ & $4323 \pm 52$ & $1732 \pm 31$ & $6055 \pm 61$ & $595 \pm 18$ \\
& $\mathrm{NXT} /$ Def-1 & $-2.4 \% \pm 1.2 \%$ & $-0.6 \% \pm 1.9 \%$ & $-1.9 \% \pm 1.0 \%$ & $0.4 \% \pm 3.2 \%$ \\
\hline
\end{tabular}

Table 6.1: Numbers of accepted events at both detectors for MC with default crosstalk fractions (Def) and MC with the new crosstalk fractions (NXT). The oscillation parameters are $\left|\Delta m_{31}^{2}\right|=2.4 \times 10^{-3} \mathrm{eV}^{2}, \sin ^{2} 2 \theta_{13}=0.15, \sin ^{2} 2 \theta_{23}=1$. The numbers are normalized to FD POT $=3.25 \times 10^{20}$ (2-year exposure) and ND POT $=1 \times 10^{19}$. The errors are only statistical errors. 


\subsection{Summary}

In this chapter, we have demonstrated that the low pulse-height hits are not well modeled in the MC simulation and the older $\nu_{e}$ selection algorithm was quite sensitive to the low pulse-height hits. We also describe our effects in tuning the crosstalk to better match the data. In order to make the $\nu_{e}$ selection algorithm less sensitive to the low pulse-height hits, we made several changes to the $\nu_{e}$ analysis, including removing hits below $2 \mathrm{PE}$ at analysis time. After making the modifications, we showed that the $\nu_{e}$ selection algorithm is no longer sensitive to the detailed modeling of crosstalk hits. The systematic error from crosstalk modeling is on the order of only $1-2 \%$. 


\section{Chapter 7}

\section{Near Detector Background Decomposition}

\section{$7.1 \quad$ Introduction}

In order to obtain the optimal sensitivity on $\theta_{13}$, it is crucial to understand the uncertainties in the background estimate in the $\nu_{e}$ appearance analysis. If $\theta_{13}$ is at the CHOOZ limit, the expected signal to background ratio at the Far Detector is roughly 1:3. The Far Detector background prediction is obtained through extrapolation from the Near Detector. In principle, we can measure the background rate in the ND and predict the FD background rate by multiplying the ND background rate by the Far/Near ratio which is calculated using MC. The advantage of this extrapolation approach is that a lot of uncertainties cancel to a large extent since they are present at both detectors.

In practice, however, the extrapolation to the FD is complex for the $\nu_{\mu} \rightarrow \nu_{e}$ analysis because different background sources extrapolate differently. The $\nu_{\mu}$ CC background is suppressed in the FD because of $\nu_{\mu} \rightarrow \nu_{\tau}$ oscillation while the NC background is unaffected by the oscillation. Also the beam $\nu_{e}$ components are slightly 
different at the two detectors because of different detector solid angles. Therefore, some knowledge about the relative contribution from different background sources is necessary. In this Chapter, we describe a data-driven method that can be used to obtain relative $\nu_{\mu} \mathrm{CC}$ and $\mathrm{NC}$ background contributions in the ND from comparison of background rates in the horn-on and horn-off configurations. These two configurations give significantly different ratios of $\nu_{\mu} \mathrm{CC}$ to $\mathrm{NC}$ backgrounds and thus a comparison of background levels can yield information regarding relative contributions from these two sources.

\subsection{Horn-on and Horn-off Spectra}

The neutrinos in the NuMI beam originate from decays of focused mesons (pions and kaons) in the decay pipe. The mesons produced in the MINOS graphite target are focused by two magnetic horns. The nominal horn current is $185 \mathrm{kA}$ in each horn. Suppose the meson emanates from the target at a polar angle $\theta$, we have

$$
\tan \theta=\frac{p_{T}}{p_{z}}
$$

where $p_{T}$ and $p_{z}$ are the transverse and longitudinal momenta of the meson. In highenergy collisions the average transverse momentum of outgoing particles is known to be almost independent of the nature of the incoming particles and of the interaction energy. It is also generally good approximation to assume that the average transverse momentum does not depend on the angle of emission of secondaries ${ }^{1}$. For the secondaries produced in the proton-nucleus collisions inside the MINOS target, $\left\langle p_{T}\right\rangle \sim 350 \mathrm{MeV} / c$, so the meson longitudinal momentum and angle are inversely

\footnotetext{
${ }^{1}$ Many experiments show that for a specific secondary particle the average transverse momentum may vary moderately with the longitudinal momentum of that particle, e.g. [144], which is known as the seagull effect. This effect was briefly discussed in Chapter 5 for neutrino induced interactions.
} 
related:

$$
p_{z} \simeq p_{T} / \theta
$$

As a result, fast mesons exit the target at small angles and can pass through the field-free aperture ("neck") of the horn, while slower mesons exit the target at larger angles and enter the horn field region to get focused.

For a given momentum and distance from a target, the required transverse momentum $\left(p_{\perp}\right)$ kick from the horn to focus the pion parallel to the beam axis grows linearly with radius. Since the magnetic field is falling as $1 / R$, the path length through the horn is required to grow as $R^{2}$, i.e. the inner conductor should be parabolic. Therefore the horns designed for MINOS have parabolic shape.

Fig.7.1 shows the ND spectrum, broken up into 5 categories of focused mesons:

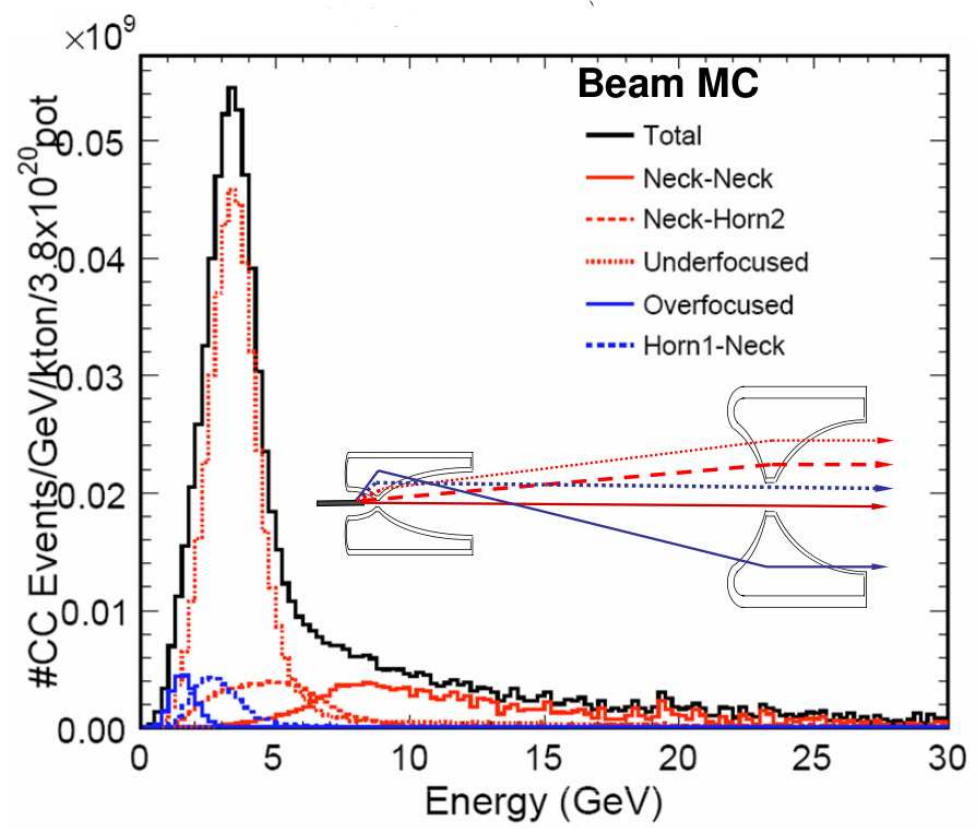

Figure 7.1: Breakdown of ND neutrino spectrum in the low energy (LE) beam configuration. Diagram shows the 5 general trajectories of mesons that pass through the horns. (Plot courtesy Zarko Pavlovic) 
(1) Neck-Neck Very high energy mesons pass through the necks of both horns at small angles, contributing to the high energy tail.

(2) Neck-Horn2 Slightly softer mesons exit the target at small enough angle to pass through the neck of horn 1 , but are focused by horn 2 .

(3) Underfocused Softer mesons emitted at larger angles are underfocused by horn 1 (i.e. still diverge as they exit horn 1 ), but are focused by horn 2 (this category constitutes the vast majority of the flux in the low energy (LE) beam).

(4) Horn1-Neck Still softer mesons are focused entirely by horn 1 and pass through the neck of horn 2.

(5) Overfocused The softest mesons are overfocused by horn 1 but properly focused by horn 2 .

If we turn off the two magnetic horns, the low energy pions will not get focused thus the peak in the neutrino energy spectrum will disappear, but the high energy tail will remain almost unchanged (Neck-Neck in Fig.7.1). Fig.7.2 shows the MC energy spectra for two different horn current configurations: 185kA (horn-on) and 0kA (horn-off).

If we apply the same $\nu_{e}$ analysis cuts (pre-selection cuts and ANN PID cut, details in Chapter 4) to the ND horn-off sample, one would expect the number of $\nu_{\mu} \mathrm{CC}$ and beam $\nu_{e}$ events that pass the $\nu_{e}$ cuts to be significantly reduced since the neutrino flux in the energy range of interest $(1 \mathrm{GeV}$ to $8 \mathrm{GeV})$ is highly suppressed in the horn-off configuration. Therefore the dominant $\nu_{e}$ background in the horn-off sample are the NC interactions. This NC-enriched sample can be used to understand the relative $\nu_{\mu} \mathrm{CC}$ and $\mathrm{NC}$ background contributions in the horn-on sample. In February 2006 and July 2007, the MINOS experiment took several days of horn-off data to facilitate the $\nu_{e}$ analysis. In total, $5.5 \times 10^{18}$ protons on target were delivered to the 


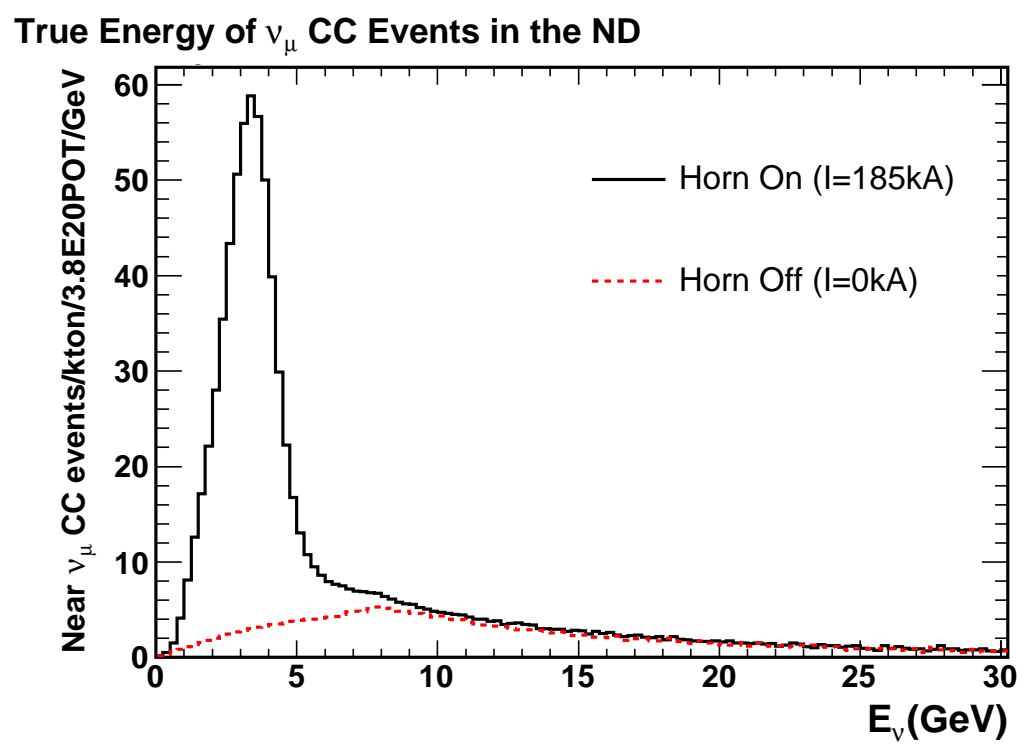

Figure 7.2: The simulated $\nu_{\mu} \mathrm{CC}$ event rate as a function of $E_{\nu}$ in the ND with two different horn current configurations: black-185kA, red-0kA.

MINOS target during the horn-off data run (roughly 9 days altogether). Fig.7.3 shows the reconstructed energy spectra of data and $\mathrm{MC}$ events that pass the $\nu_{e}$ selection algorithm for the horn-on (left) and horn-off (right) configurations. Table 7.1 shows the number of events that pass the $\nu_{e}$ selection for the two configurations. The fraction of $\mathrm{NC}$ events goes from $65 \%$ to $87 \%$ if we turn off the horns. We also observe a reduction in the number of events for neutrinos whose true energy is below $10 \mathrm{GeV}$ when we turn off the horns; however, the number of interactions remain almost the same for neutrinos whose energy is above $10 \mathrm{GeV}$. These observations are consistent with what we expect from the neutrino fluxes shown in Fig.7.2. 

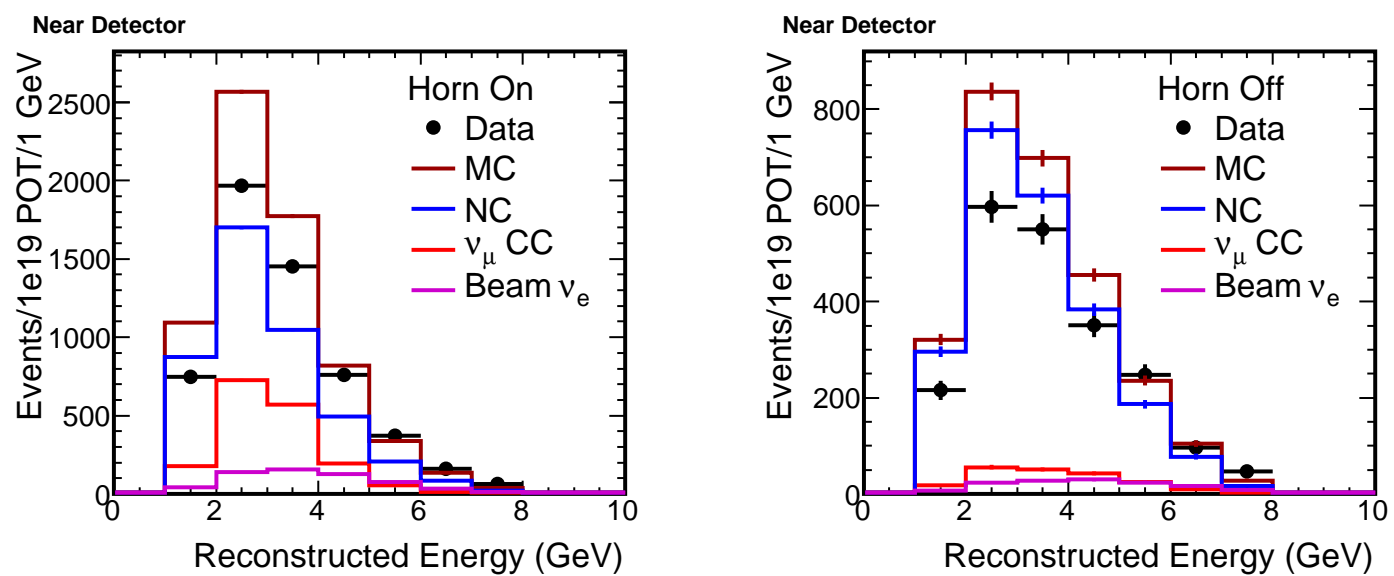

Figure 7.3: The reconstructed energy spectra of events that pass the $\nu_{e}$ selection algorithm. Left: horn-on; Right: horn-off. All spectra are normalized to $1 \times 10^{19}$ POTs.

\subsection{ND $\nu_{e}$ Background Decomposition}

The horn-on and horn-off configurations give significantly different ratios of $\nu_{\mu} \mathrm{CC}$ to $\mathrm{NC}$ backgrounds. By comparing the background rates in the two configurations, one may learn useful information regarding relative contributions from those two sources. In this section, we will show how we decompose the background components and evaluate systematic errors on our results.

We can write the total background as a sum of the individual components:

$$
\begin{aligned}
N^{o n} & =N_{N C}+N_{C C}+N_{e} \\
N^{o f f} & =r_{N C} \cdot N_{N C}+r_{C C} \cdot N_{C C}+r_{e} \cdot N_{e}
\end{aligned}
$$

where

$$
r_{N C}=\frac{N_{N C}^{o f f}}{N_{N C}}, r_{C C}=\frac{N_{C C}^{o f f}}{N_{C C}}, r_{e}=\frac{N_{e}^{o f f}}{N_{e}}
$$

$N^{o n}$ and $N^{o f f}$ are the total numbers of background events with the horns turned 
All Events

\begin{tabular}{|c|c|c|c|c|c|}
\hline & $\mathrm{NC}$ & $\nu_{\mu} \mathrm{CC}$ & Beam $\nu_{e}$ & Total MC & Data \\
\hline horn-on & $4429(65 \%) \pm 17$ & $1742(26 \%) \pm 10$ & $593(9 \%) \pm 6$ & $6764 \pm 21$ & $5524 \pm 35$ \\
\hline horn-off & $2338(87 \%) \pm 31$ & $205(8 \%) \pm 9$ & $137(5 \%) \pm 7$ & $2680 \pm 36$ & $2105 \pm 62$ \\
\hline
\end{tabular}

True $E_{\nu}<10 \mathrm{GeV}$

\begin{tabular}{|c|c|c|c|c|}
\hline & $\mathrm{NC}$ & $\nu_{\mu} \mathrm{CC}$ & Beam $\nu_{e}$ & Total MC \\
\hline horn-on & $3308 \pm 15$ & $1734 \pm 10$ & $593 \pm 6$ & $5635 \pm 19$ \\
\hline horn-off & $1244 \pm 23$ & $199 \pm 9$ & $137 \pm 7$ & $1580 \pm 26$ \\
\hline
\end{tabular}

True $E_{\nu}>10 \mathrm{GeV}$

\begin{tabular}{|c|c|c|c|c|}
\hline & $\mathrm{NC}$ & $\nu_{\mu} \mathrm{CC}$ & Beam $\nu_{e}$ & Total MC \\
\hline horn-on & $1121 \pm 9$ & $8 \pm 1$ & $0.4 \pm 0.2$ & $1130 \pm 9$ \\
\hline horn-off & $1093 \pm 21$ & $6 \pm 2$ & $0.3 \pm 0.3$ & $1099 \pm 22$ \\
\hline
\end{tabular}

Table 7.1: Number of events that are accepted as $\nu_{e}$ candidates. Top: all events, comparison of data and MC; Middle: MC events $E_{\nu}<10 \mathrm{GeV}$; Bottom: MC events $E_{\nu}>10 \mathrm{GeV}$. All numbers represent $1 \times 10^{19}$ POTs exposure.

on/off, which we measure in the ND. $N_{N C}^{(o f f)}, N_{C C}^{(o f f)}, N_{e}^{(o f f)}$ are the MC predictions of the NC, $\nu_{\mu} \mathrm{CC}$ and beam $\nu_{e}$ backgrounds when the horns are turned on (off). We will take the three ratios (7.5) and beam $\nu_{e}$ background $\left(N_{e}\right)$ from MC simulation. We do not trust the MC prediction of individual background component in each beam configuration due to lack of knowledge of neutrino interactions in the few-GeV range. However, the ratios $r_{N C}, r_{C C}$ and $r_{e}$ are well modeled and quite robust since a lot of uncertainties cancel in the ratio. Now we have two equations (7.3) and (7.4) with two unknown variables $N_{N C}$ and $N_{C C}$ which can be solved as:

$$
\begin{aligned}
N_{C C} & =\frac{r_{N C} N^{o n}-N^{o f f}+\left(r_{e}-r_{N C}\right) N_{e}}{r_{N C}-r_{C C}} \\
N_{N C} & =\frac{N^{o f f}-r_{C C} N^{o n}-\left(r_{e}-r_{C C}\right) N_{e}}{r_{N C}-r_{C C}}
\end{aligned}
$$


The systematic errors on $N_{C C}$ and $N_{N C}$ can be written as:

$$
\begin{aligned}
\delta N_{C C}^{2} & =\left(\frac{r_{N C}}{r_{N C}-r_{C C}}\right)^{2}\left(\delta N^{o n}\right)^{2}+\left(\frac{1}{r_{N C}-r_{C C}}\right)^{2}\left(\delta N^{o f f}\right)^{2}+\left(\frac{r_{e}-r_{N C}}{r_{N C}-r_{C C}}\right)^{2} \delta N_{e}^{2} \\
& +\left(\frac{N_{N C}}{r_{N C}-r_{C C}}\right)^{2} \delta r_{N C}^{2}+\left(\frac{N_{C C}}{r_{N C}-r_{C C}}\right)^{2} \delta r_{C C}^{2}+\left(\frac{N_{e}}{r_{N C}-r_{C C}}\right)^{2} \delta r_{e}^{2} \\
\delta N_{N C}^{2} & =\left(\frac{r_{C C}}{r_{N C}-r_{C C}}\right)^{2}\left(\delta N^{o n}\right)^{2}+\left(\frac{1}{r_{N C}-r_{C C}}\right)^{2}\left(\delta N^{o f f}\right)^{2}+\left(\frac{r_{e}-r_{C C}}{r_{N C}-r_{C C}}\right)^{2} \delta N_{e}^{2} \\
& +\left(\frac{N_{N C}}{r_{N C}-r_{C C}}\right)^{2} \delta r_{N C}^{2}+\left(\frac{N_{C C}}{r_{N C}-r_{C C}}\right)^{2} \delta r_{C C}^{2}+\left(\frac{N_{e}}{r_{N C}-r_{C C}}\right)^{2} \delta r_{e}^{2}
\end{aligned}
$$

There are six sources of systematic errors: the statistical error on horn-on data, the statistical error on horn-off data, the systematic error on the beam $\nu_{e}$ background, and the systematic errors on the three ratios $r_{N C}, r_{C C}$, and $r_{e}$.

Now we demonstrate this method with an example. From Table 7.1 we have:

$$
\begin{aligned}
N^{o n} & =5524 \pm 35 \\
N^{\text {off }} & =2105 \pm 62 \\
N_{e} & =593
\end{aligned}
$$

We can also calculate the horn off/on ratios based on the numbers in Table 7.1:

$$
\begin{aligned}
r_{N C} & =0.528 \\
r_{C C} & =0.117 \\
r_{e} & =0.232
\end{aligned}
$$

We will evaluate the systematics more thoroughly later in this chapter. For now we assume a $15 \%$ systematic error on the $\nu_{e}$ background and a $10 \%$ error on all the three ratios: $\delta N_{e} / N_{e}=15 \%, \delta r_{C C} / r_{C C}=10 \%, \delta r_{N C} / r_{N C}=10 \%$, and $\delta r_{e} / r_{e}=10 \%$. 
We plug these numbers into Eqs (7.6-7.9) and get the following results:

$$
\begin{aligned}
N_{C C} & =1548 \\
N_{N C} & =3383 \\
\left|\delta N_{C C}\right| & =\sqrt{44.8^{2}+150.1^{2}+64.1^{2}+434.6^{2}+44.1^{2}+33.5^{2}} \simeq 470 \\
\left|\delta N_{N C}\right| & =\sqrt{9.9^{2}+150.1^{2}+24.9^{2}+434.6^{2}+44.1^{2}+33.5^{2}} \simeq 464 .
\end{aligned}
$$

We have $\delta N_{C C} / N_{C C}=30.4 \%, \delta N_{N C} / N_{N C}=13.7 \%$, i.e. we have a $14 \%$ systematic error on the $\mathrm{NC}$ background estimation and a $30 \%$ systematic error on the $\nu_{\mu} \mathrm{CC}$ background estimation. The systematic errors are dominated by the error on the horn off/on NC background ratio $r_{N C} . \delta N_{C C}$ and $\delta N_{N C}$ are almost the same which is result of the fact that $\mathrm{NC}$ and $\nu_{\mu} \mathrm{CC}$ backgrounds are highly anti-correlated:

$$
\delta N^{o n}=\delta N_{C C}+\delta N_{N C}+\delta N_{e}
$$

$\delta N^{o n}$ is very small since we have taken a lot of ND horn-on data so the statistical error on that is small. $\delta N_{e}$ is also very small since the contribution of beam $\nu_{e}$ events to the total background is small. Therefore we have

$$
\delta N_{N C} \simeq-\delta N_{C C}
$$

\subsection{Background Systematics}

We now try to obtain the energy spectra of the $\mathrm{NC}$ and $\nu_{\mu} \mathrm{CC}$ backgrounds using the horn-on/off method. We divide the reconstructed energy spectra into $1 \mathrm{GeV}$ bins and for each bin we calculate the expected numbers of $\mathrm{NC}$ and $\nu_{\mu} \mathrm{CC}$ background events using Eqs.(7.6) and (7.7) using the information on beam $\nu_{e}$ background, $r_{N C}, r_{C C}$, and 
$r_{e}$ taken from MC. First we examine the various sources of systematics in Eqs.(7.8) and (7.9): data statistics, beam $\nu_{e}$ background systematics, and uncertainties in the horn-off/on ratios.

\subsubsection{Data (Horn-on/off) Statistics}

In principle we can use all the ND horn-on data to shrink the horn-on data statistics. But for the first MINOS $\nu_{e}$ analysis, we decided to use approximately one sixth of the ND data taken between May 2005 and July 2007 (run I and run II periods). The reason is that the $1 / 6$ sample $\left(4.55 \times 10^{19}\right.$ POTs in total $)$ already gives a very small statistical error and it is less time-consuming to analyze the $1 / 6$ sample. We do sample over the whole period to track the temporal variations of the detector response and other changes.

We use all the available horn-off data taken in 2006 and $2007\left(5.52 \times 10^{18}\right.$ POTs in total). According to Eqs.(7.19) and (7.20), the horn-off data statistical error is the second largest contribution to the total systematic error. However, the total systematic error is dominated by the uncertainties in the horn off/on ratio, specifically $r_{N C}$, which will be discussed later.

\subsubsection{Beam $\nu_{e}$ Background}

The number of beam $\nu_{e} \mathrm{CC}$ events is taken from the MC as one of the inputs to the horn-on/off background decomposition method. The (anti-)electron neutrinos in the 
NuMI beam are produced by muon, pion and kaon decays:

$$
\begin{aligned}
\pi^{+} \rightarrow & \mu^{+}+\nu_{\mu} \\
& \mu^{+} \rightarrow \bar{\nu}_{\mu}+e^{+}+\nu_{e} \\
K^{+} \rightarrow & \pi^{0}+e^{+}+\nu_{e}\left(\text { Called } K_{e 3}^{+}\right) \\
K_{L}^{0} \rightarrow & \left.\pi^{ \pm}+e^{\mp}+\nu_{e}\left(\bar{\nu}_{e}\right) \text { (Called } K_{e 3}^{0}\right)
\end{aligned}
$$

Here we do not list other decay modes that produce (anti-)electron neutrinos $\left(\pi^{+} \rightarrow\right.$ $\nu_{e}, \pi^{-} \rightarrow \bar{\nu}_{e}, \pi^{-} \rightarrow \mu^{-} \rightarrow \bar{\nu}_{e}$ and $\left.K^{-} \rightarrow \bar{\nu}_{e}\right)$, but they are included in the MC. Fig.7.4 shows the true energy spectra of ND beam $\nu_{e}$ events broken up as different parent types. The peak in the energy spectrum is dominated by the $\mu^{+}$decays and the high energy tail is dominated by the kaon decays. The $\nu_{e}$ selection cuts preferentially select low energy beam $\nu_{e}$ events which are mostly from $\mu^{+}$decays. The main error on the beam $\nu_{e}$ background is from the neutrino flux simulation which includes the modeling of hadron production at target, horn focusing, and beam-line materials (decay pipe etc.). Since $\mu^{+}$is the decay product of the $\pi^{+}$decay, and we can constrain $\pi^{+}$ production at target reasonably well using ND $\nu_{\mu} \mathrm{CC}$ data, the error on the beam $\nu_{e}$ flux is expected to be small. The flux error for the beam $\nu_{e}$ background is estimated to be around $10 \%$. Other systematic errors include the uncertainties in the neutrino cross section, detector calibration, and the simulation of event topology etc. and these errors are quite small compared with the flux error. We assign a $15 \%$ systematic error to the number of beam $\nu_{e}$ background which is a relatively conservative error estimation. 

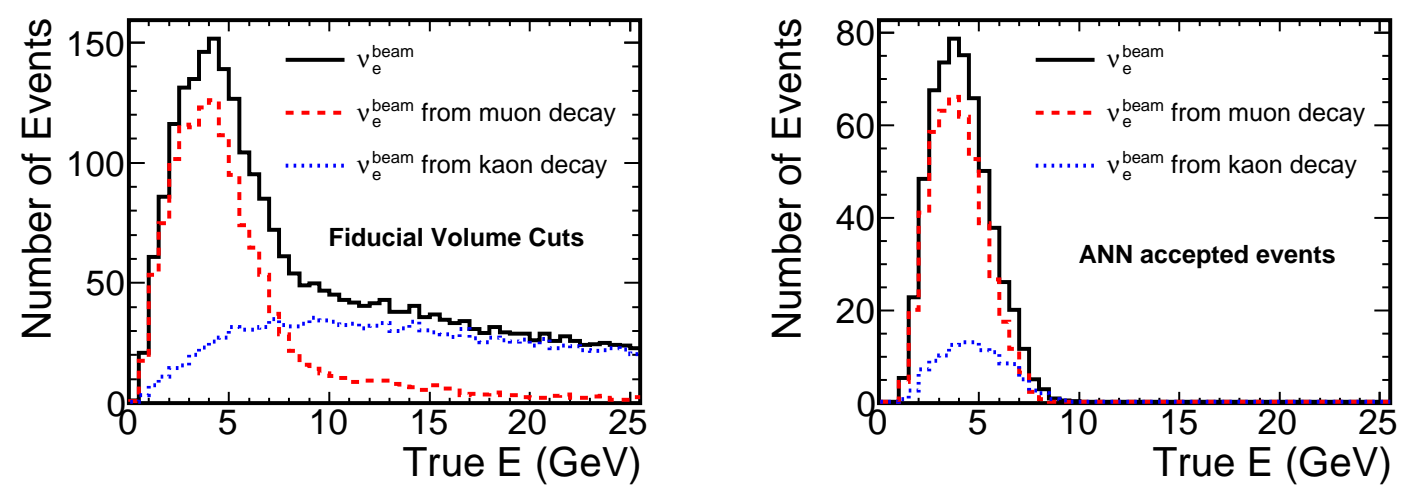

Figure 7.4: The true energy spectra of ND beam $\nu_{e}$ events. Left: events passing fiducial volume cuts; Right: events passing all $\nu_{e}$ selection cuts. All spectra are normalized to $1 \times 10^{19}$ POTs.

\subsubsection{Uncertainties in the Horn-off/on Ratios}

The ND background systematic error is dominated by the uncertainties in the hornoff/on ratios (7.5), specifically $r_{N C}$. Fig.7.3 shows that the MC does not reproduce data very well. The $\nu_{e}$ selection algorithm only accepts a small fraction of the neutrino events in the MINOS detectors (1\% of all the $\nu_{\mu} \mathrm{CC}$ events and $7 \%$ of all the $\mathrm{NC}$ events which are the dominant backgrounds). Large fraction of these events are consistent with electromagnetic cascades and usually there are one or multiple $\pi_{0}$ 's in the final state. It turns out that the systematic uncertainties in the modeling of these peculiar events are rather large. The simulation of these events is very sensitive to many factors which include the modeling of the event topology (e.g. hadronization model and intra-nuclear rescattering model) and the detector response (e.g. crosstalk modeling). We have discussed some of these effects in the previous chapters. It is not surprising that our MC prediction deviates from data by approximately $20 \%$. However, if we take the ratio of the horn-off spectrum to the horn-on spectrum, a lot of uncertainties cancel. Therefore the ratio is more robust than the individual spectra. Fig.7.5 shows the horn off/on ratios in bins of reconstructed energy. The 
error bars represent the MC statistics. As energy goes up, the ratios approach 1 since the high energy horn-on and horn-off fluxes are almost the same.
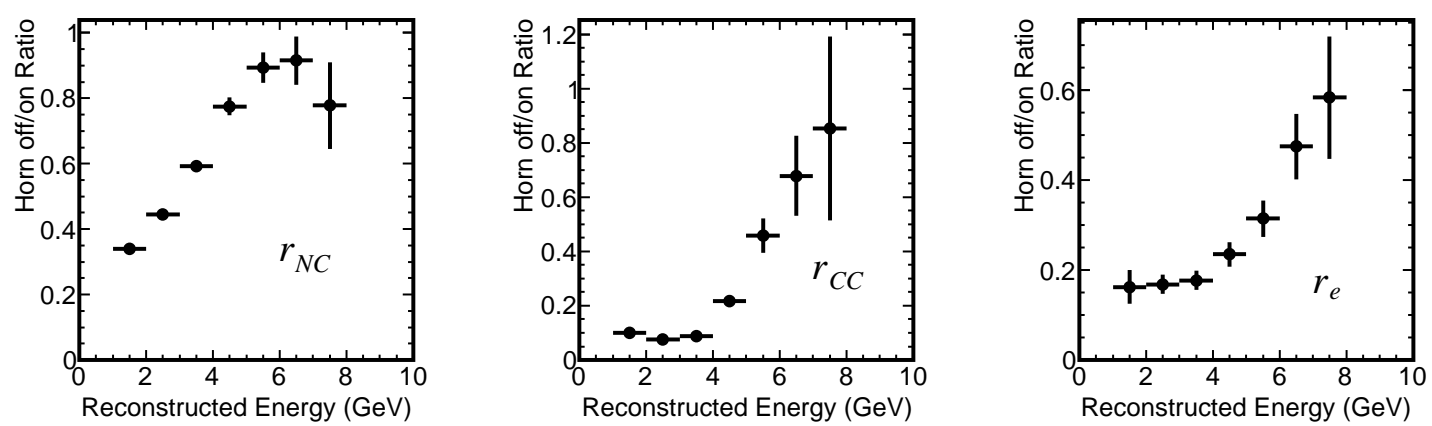

Figure 7.5: The ND horn-off over horn-on spectrum ratios. The error bars represent the MC statistics.

We will show that the ratio $r_{N C}$ is quite robust by demonstrating the following two points (the same arguments apply to $r_{C C}$ and $r_{e}$ ):

1. $r_{N C}$ is well modeled in the fiducial volume sample.

2. $r_{N C}$ is almost the same in the fiducial volume, pre-selection, and $\nu_{e}$-selected samples.

Fig.7.6(a) shows $r_{N C}$ in bins of reconstructed energy after fiducial volume cuts. The ratios are from events selected by NOT- $\nu_{\mu}$ PID ${ }^{2}$ which primarily selects NC events. The black points are from data events while the red points are from MC events. Data and MC agree quite well for the fiducial volume sample. Fig.7.6(b) shows $r_{N C}$ from true NC events after the fiducial volume cuts (black), after the preselection cuts (blue), and after the ANN PID cut (red). It is clear that $r_{N C}$ does not change much as we apply successive cuts. From those two plots we can conclude that $r_{N C}$ is well modeled in the $\nu_{e}$-selected sample since $r_{N C}$ is well modeled in the

\footnotetext{
${ }^{2}$ This is the inverse of the PID developed for the MINOS $\nu_{\mu} \mathrm{CC}$ disappearance analysis.
} 


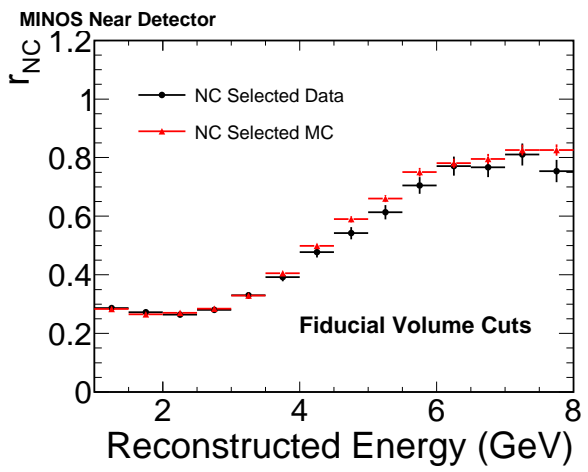

(a)

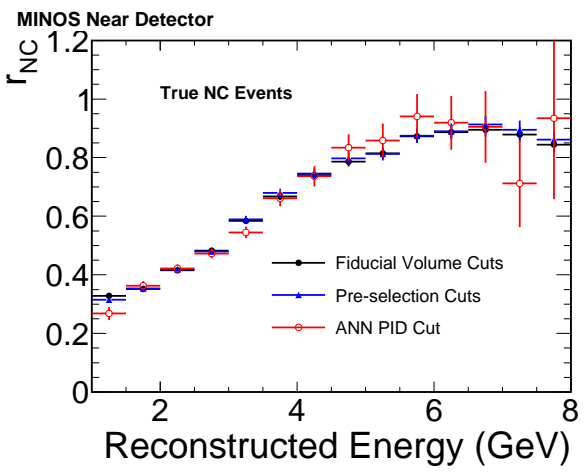

(b)

Figure 7.6: $r_{N C}$ in bins of reconstructed energy. (a) After fiducial volume cuts. The black points are from NOT- $\nu_{\mu} \mathrm{CC}(\mathrm{NC})$ selected data events. The red points are from NOT $-\nu_{\mu}$ CC (NC) selected Monte Carlo events. (b) From true NC events. The black points are after the fiducial volume cuts. The blue points are after the pre-selection cuts. The red points are after the ANN PID cut. The error bars represent the MC statistics.

fiducial volume sample where MC predictions can be verified by the data and it does not change much as we apply the $\nu_{e}$ selection cuts.

It is worth noting that the ratios are quite different between Fig.7.6(a) and Fig.7.6(b). The reason is that the NOT- $\nu_{\mu}$ PID does not select a very pure NC sample. It is practically impossible to separate $\mathrm{NC}$ events from $\nu_{\mu} \mathrm{CC}$ events where the muon momentum is so low that the muon track is invisible in the hadronic shower. The $\nu_{\mu}$ PID was tuned to select a very pure $\nu_{\mu} \mathrm{CC}$ sample, therefore there is a big contribution of $\nu_{\mu} \mathrm{CC}$ background in the NOT- $\nu_{\mu}$ PID selected sample. For a fixed reconstructed energy (visible energy), the true neutrino energy of NC events is higher than that of CC events, therefore, $r_{N C}$ is higher than $r_{C C}$, which is consistent with the difference between Fig.7.6(a) and Fig.7.6(b). However, we still believe that we model things pretty well in the fiducial volume since the spectra are determined primarily by neutrino flux at this level and we understand the neutrino flux very well using the $\mathrm{ND} \nu_{\mu} \mathrm{CC}$ data. 
Now we provide a physics-motivated explanation for the fact that $r_{N C}$ is the same in the fiducial volume, pre-selection, and $\nu_{e}$-selected samples [145]. Fig.7.7(a) shows

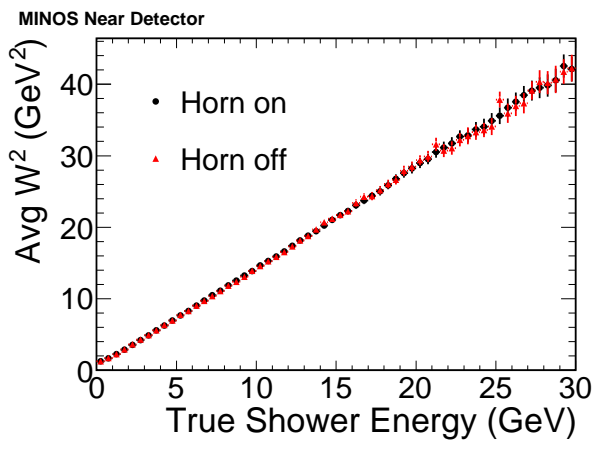

(a) $\operatorname{Avg} W^{2}$ vs $E_{s h w}$

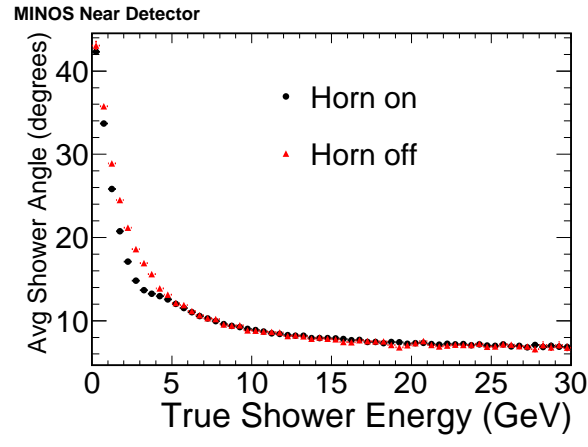

(b) Avg Shower Angle vs $E_{s h w}$

Figure 7.7: (a) Average $W^{2}$ as a function of true shower energy for events in the horn-on (black) and horn-off (red) configurations. (b) Average shower angle as a function of true shower energy for events in the horn-on (black) and horn-off (red) configurations. Fiducial volume cuts are applied.

the average $W^{2}$ as a function of true shower energy for events in the horn-on and horn-off configurations. For a fixed shower energy, events in the horn-on beam and horn-off beam can have very different incoming neutrino energy distributions. However, Fig.7.7(a) shows the $W$ distributions probed in both cases follow each other very closely, which indicates for a given true shower energy, both samples have the same $W$ distributions. As a consequence, one would expect the distributions of other kinematic variables such as $x$ (Bjorken scaling variable) and $Q^{2}$ (invariant 4-momentum transfer) are quite similar in both cases as well. The kinematic variable $W$, together with the charge of the hadronic system, completely determine the final state particle distributions of the hadronic system in the hadronic center of mass frame. The fact that the showers are equivalent in the center of mass, and the shower energy in the lab is the same, means that the Lorentz boost to the lab is the same in both cases.

There does, however, remain a significant difference arising from the scattering kinematics. Fig.7.7(b) shows the average shower angle as a function of true shower 
energy for events in the horn-on and horn-off configurations. The shower angle is defined as the direction of total hadronic shower momentum $P_{\text {had }}$ with regards to the neutrino beam direction. The showers in the two cases emerge at very different angles to the direction of the incident neutrino beam for $E_{s h w}<5 \mathrm{GeV}$. This can be seen from the fact that $Q^{2}=4 E E^{\prime} \sin ^{2}\left(\theta_{l e p} / 2\right)$, so although $Q^{2}$ will be the same, $\theta$ can be quite different since $E$ is quite different at low energies (here $\theta_{\text {lep }}$ is the lepton angle). Fig.7.8 shows the probability of events to pass the ANN $\nu_{e}$ selection as a function of the shower angle for events with true shower energy between 3 and $4 \mathrm{GeV}$. The selection efficiency is essentially flat over the region where most events occur, $\cos \left(\theta_{z}\right)>0.90$. Since the $\nu_{e}$ selection is to first order insensitive to the shower angle, the ratio $r_{N C}$ does not change as selection cuts are applied. This together with the fact that $W$ distributions are quite similar in the horn-on/off beams for a fixed shower energy explain why $r_{N C}$ is almost the same in the fiducial volume, pre-selection, and $\nu_{e}$-selected samples.

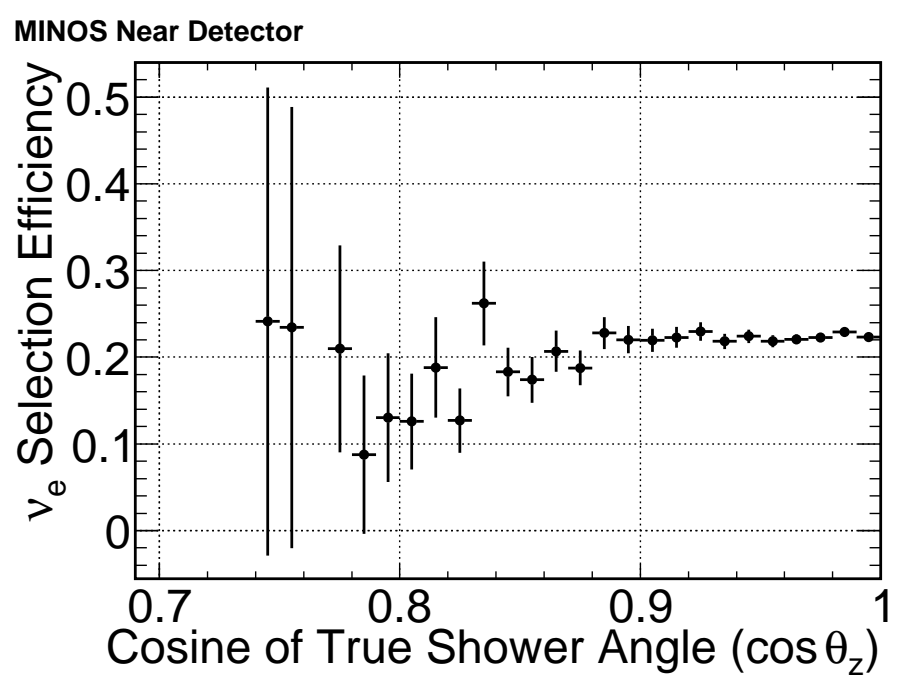

Figure 7.8: Selection efficiency for ANN algorithm as a function of the shower angle with respect to the beam for events with true shower energy between 3 and $4 \mathrm{GeV}$.

We now discuss various sources of systematic uncertainties and their impacts on 
the three ratios $r_{N C}, r_{C C}$, and $r_{e}$.

\section{Flux Uncertainties}

As discussed in Chapter 2, the neutrino flux is calculated using a FLUKA based simulation of the proton-target interactions and a GEANT3 based beam-line simulation. The production of secondary mesons in the NuMI target was tuned using the MINOS ND $\nu_{\mu}$ CC data, which significantly reduces the flux uncertainties. A one sigma error band, which includes uncertainties in hadronic production at the target as well as beam and target parameters (position, current, baffle scraping etc.) is obtained from the fit for the $\nu_{\mu}$ and $\bar{\nu}_{\mu}$ flux. Fig.7.9 shows the impact of the flux uncertainties on the horn off/on ratios.
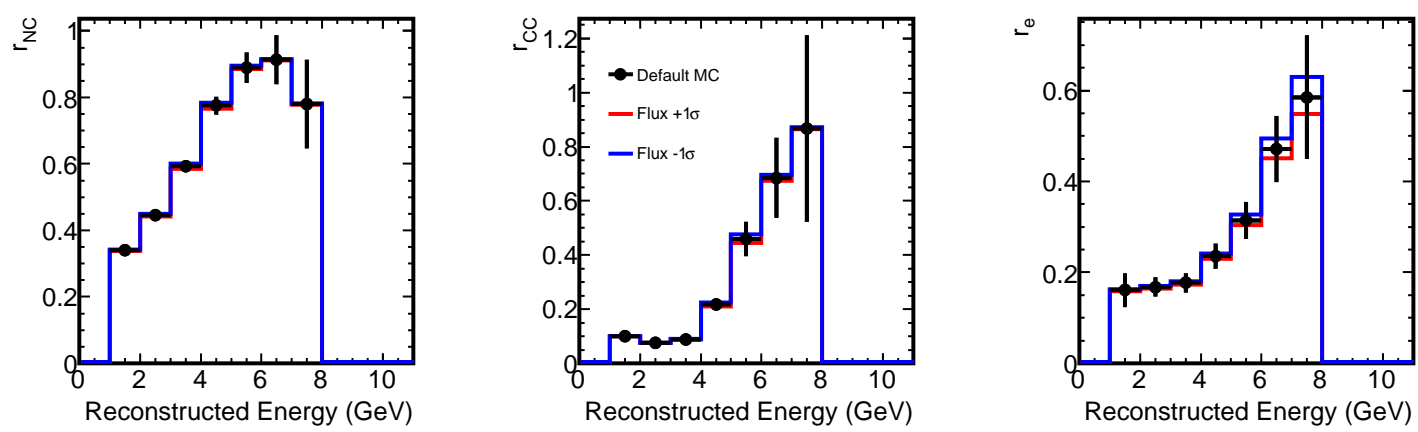

Figure 7.9: Impact of flux uncertainties in the horn off/on ratios. The error bars represent the MC statistical error.

\section{Cross Section Uncertainties}

Neutrino interactions are modeled by the NEUGEN-v3 program, which simulates both (quasi-)elastic and inelastic neutrino scattering. The latter includes a Rein-Sehgal based treatment of single pion production in neutrino induced resonance decay [102], charged and neutral-current coherent pion production and a modified leading order 
deep inelastic scattering (DIS) model extended to improve the treatment in the transition region between DIS and resonant production. As shown in Fig.4.22 (Chapter 4), the background events that pass the ANN $\nu_{e}$ selection are predominantly DIS interactions. The DIS cross section value comes from two sources: the world average value and fits carried out by MINOS to data in the 10-30 GeV energy range. We estimate the uncertainty on the cross section to be $3 \%$. However we do not use this parameter to evaluate the systematics since it is completely redundant with the flux uncertainty in the high energy tail, which is much larger.

The NEUGEN program uses two parameters to describe the cross section in the few $\mathrm{GeV}$ region (quasi-elastic and resonance production). Both refer to a dipole parameterization of the axial component of the neutrino-nucleon cross section, and both are called the axial-vector "mass" $M_{A}^{Q E}$ and $M_{A}^{\text {Res }}$. The best information on these parameters comes from neutrino-nucleus data in deuterium-filled bubble chambers. To evaluate the effects of uncertainties in the cross section relating to the quasi-elastic and resonance production, we vary $M_{A}^{Q E}$ and $M_{A}^{R e s}$ by $\pm 15 \%$. We also vary the treatment in the transition region between DIS and resonant production. The effects of those variations are shown in Fig.7.10.
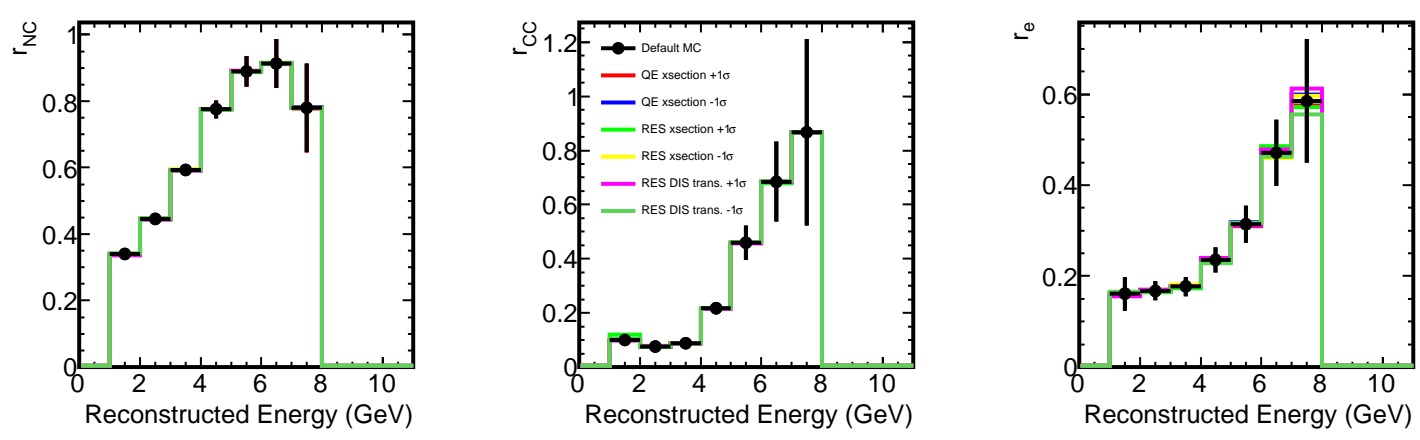

Figure 7.10: Impact of cross section uncertainties in the horn off/on ratios. The error bars represent the MC statistical error. 


\section{Hadronization Model Uncertainties}

The largest uncertainties in our Monte Carlo simulation are due to lack of knowledge of neutrino interactions in the few-GeV range, in particular the hadronization process, along with intranuclear rescattering effects. While the model we developed is tuned to external bubble chamber data, as described in Chapter 5, the existing data do not cover the kinematic range of events selected in the $\nu_{e}$ analysis. We have identified aspects of the hadronization model that are most important to the $\nu_{e}$ measurement and that are poorly constrained by the external data:

1. Baryon $x_{F}$ selection

2. Probability of $\pi^{0}$ production

3. Charged - neutral pion multiplicity correlation

4. Ambiguity in $x_{F}$ vs $p_{T}$ correlation

5. Average $p_{T}$

6. Uncertainty in angular distribution of isotropic two-body decays

7. Average particle multiplicity

The uncertainties are constrained by the external bubble chamber experiment data. The impacts of these uncertainties on the horn off/on ratios are shown in Fig.7.11.

\section{Intranuke Uncertainties}

Final-state hadrons resulting from neutrino-nucleon interactions may reinteract with other nucleons within the same nucleus before emerging. In the NEUGEN program, hadron intranuclear rescattering is handled by the program INTRANUKE which is an 

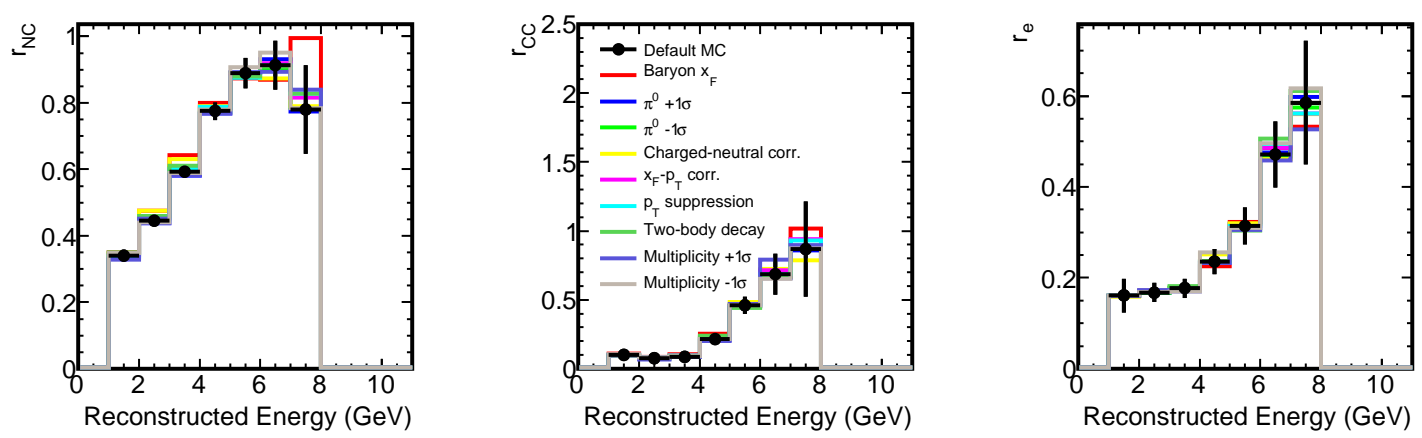

Figure 7.11: Impact of hadronization model uncertainties on the horn off/on ratios. The error bars represent the MC statistical error.

intranuclear cascade model (INC). In 2007, the hadron absorption probability was increased by roughly $10 \%$ based on external hadron-nucleus cross section measurements and data from bubble chamber experiments. However, because of timing constraints that would have been exacerbated by the need to validate the updated MC model, the current nue analysis relies on an older version of INTRANUKE that predates the above modification. This choice is justified by the fact the changes in the INTRANUKE model are not expected to affect the Far/Near extrapolation or the signal prediction significantly. To evaluate the impact of the INTRANUKE changes on the horn on/off analysis, we plot the horn off/on ratios with the default MC and with the modified MC. The results are shown in Fig.7.12.

\section{Crosstalk Modeling}

The crosstalk simulation has been shown to have poor agreement with data. Instead of regenerating Monte Carlo with improved crosstalk model described in Chapter 6, we decided to use the old Monte Carlo and make some corrections because of time constraints. We modified the $\nu_{e}$ selection algorithm to remove most of the dependence on the low pulse-height hits, namely to compute discriminating variables with hits having a raw charge above 2 photo-electrons. To evaluate the remaining 

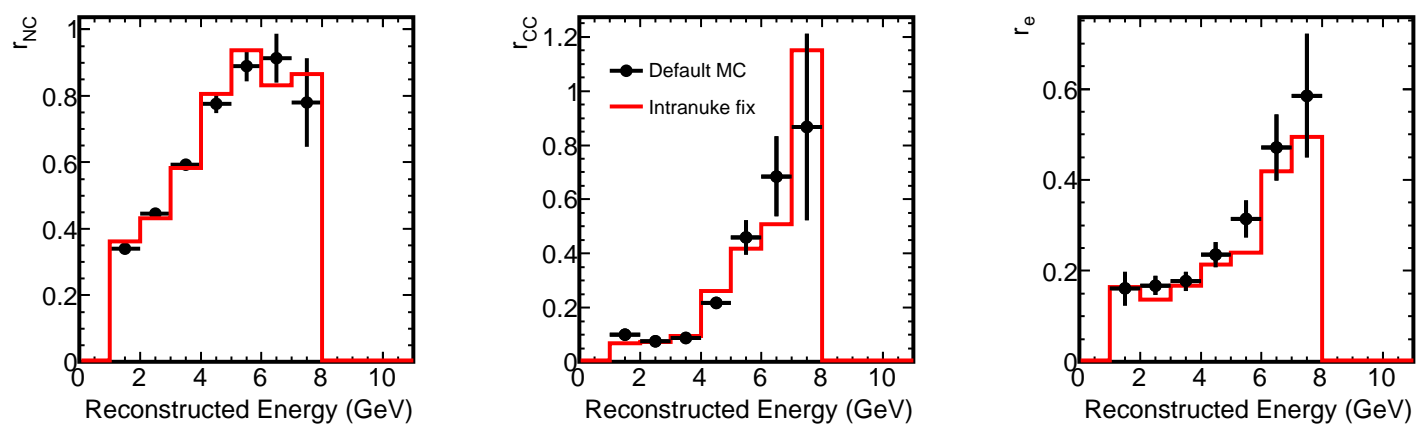

Figure 7.12: Impact of intranuke rescattering uncertainties on the horn off/on ratios. The error bars represent the MC statistical error.

effect caused by the crosstalk mismodeling, we produced a limited amount of Monte Carlo in the horn-on and horn-off configurations with the improved crosstalk model. The comparisons of horn off/on ratios with the default and improved crosstalk models are shown in Fig.7.13.
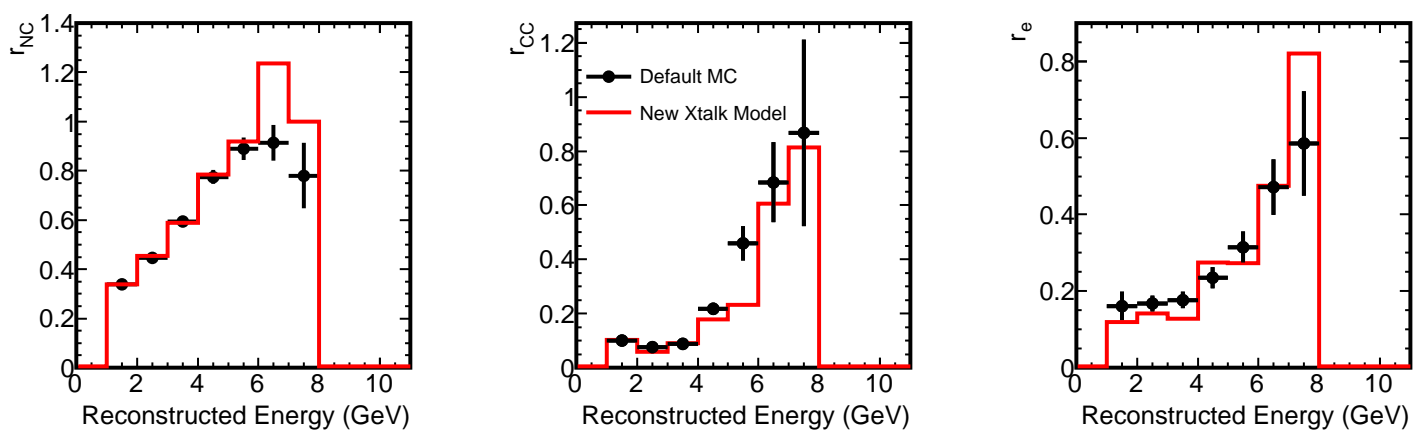

Figure 7.13: Impact of crosstalk mismodeling on the horn off/on ratios. The error bars represent the MC statistical error.

\section{Calibration}

Another important source of uncertainties is calibration. However, since the events in the horn-on/off configurations all occur in the Near Detector, a lot of calibration 
systematic errors cancel. We only expect the calibration uncertainties to change the energy scale, and from Fig.7.6 we are confident that we model the energy scale reasonably well. Thus we feel that it is not necessary to consider the systematic errors from calibration for the horn on/off method.

\section{Summary of systematics}

Tables 7.2, 7.3 and 7.4 summarize the percentage systematic errors on the horn off/on ratios $r_{N C}, r_{C C}$, and $r_{e}$ in reconstructed energy bins. The errors are calculated by taking the difference between the standard $\mathrm{MC}$ and the $\mathrm{MC}$ with alternative parameters or models. In some cases (e.g. baryon $x_{F}$, charged-neutral pion correlation), the alternative model is an overestimate of the theoretical uncertainties and we take half of the difference between the alternative $\mathrm{MC}$ and the standard $\mathrm{MC}$ as the systematic error. In other cases (e.g. intranuke), the alternative model is more consistent with what the external data suggest and we take the full difference between the alternative $\mathrm{MC}$ and the standard MC as a one-side systematic error.

Fig.7.14 shows the reconstructed energy spectra of events that pass the ANN $\nu_{e}$ selection algorithm. The systematic errors on individual spectra are quite large. But the errors cancel to a large extent so the systematic errors on the ratios are much smaller. This is seen in Fig.7.15 which shows the horn-off over horn-on spectrum ratios for $\mathrm{NC}, \nu_{\mu} \mathrm{CC}$, and beam $\nu_{e}$ events that pass the $\mathrm{ANN} \nu_{e}$ selection algorithm.

\subsubsection{Calculation of the ND NC and CC Background Energy Spectra}

The Near Detector NC and $\nu_{\mu} \mathrm{CC}$ background contributions are estimated with Eqs.(7.6) and (7.7) in bins of reconstructed energy assuming a 15\% error on the 


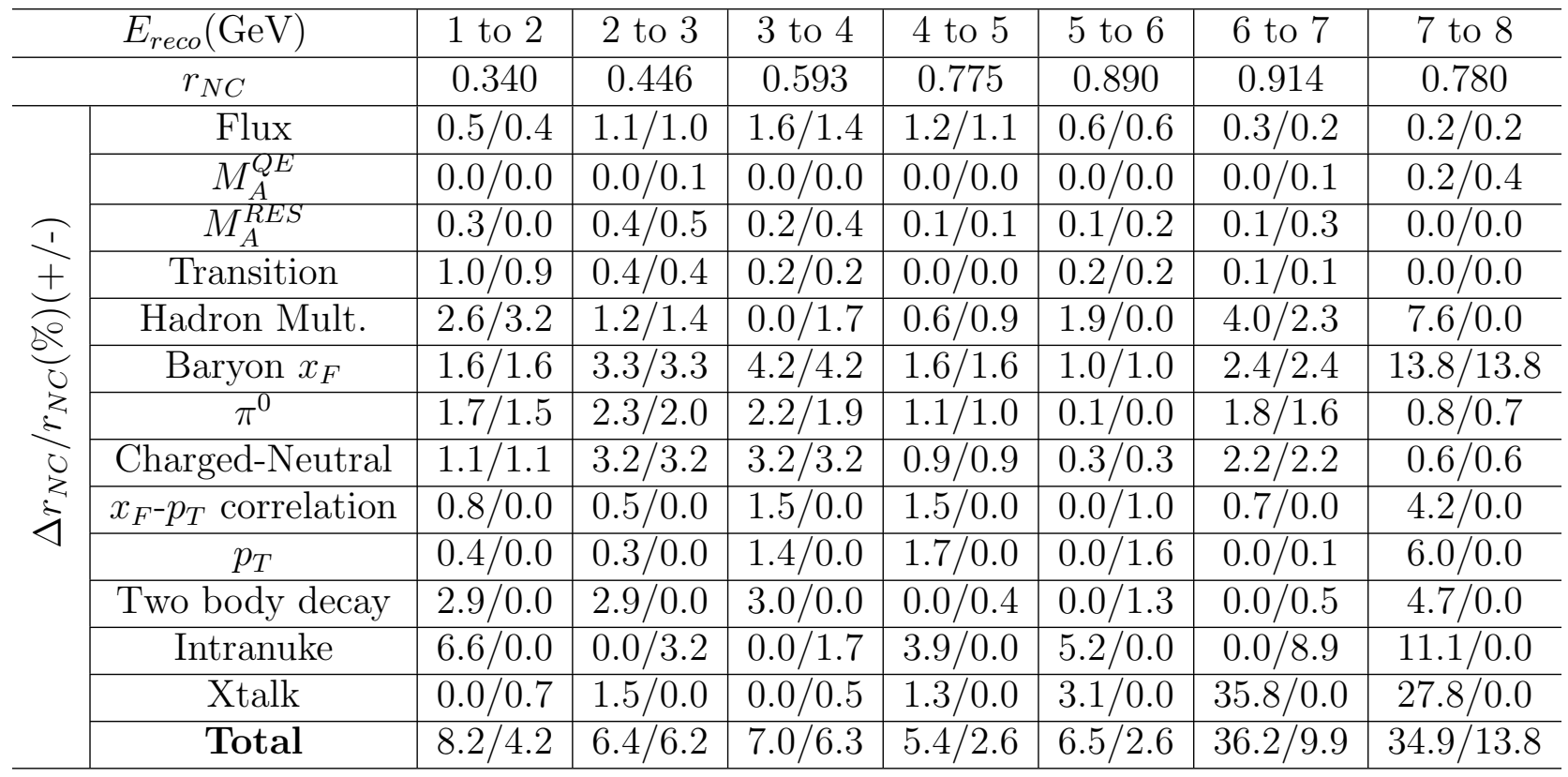

Table 7.2: Percentage systematic errors on $r_{N C}$ in reconstructed energy bins.

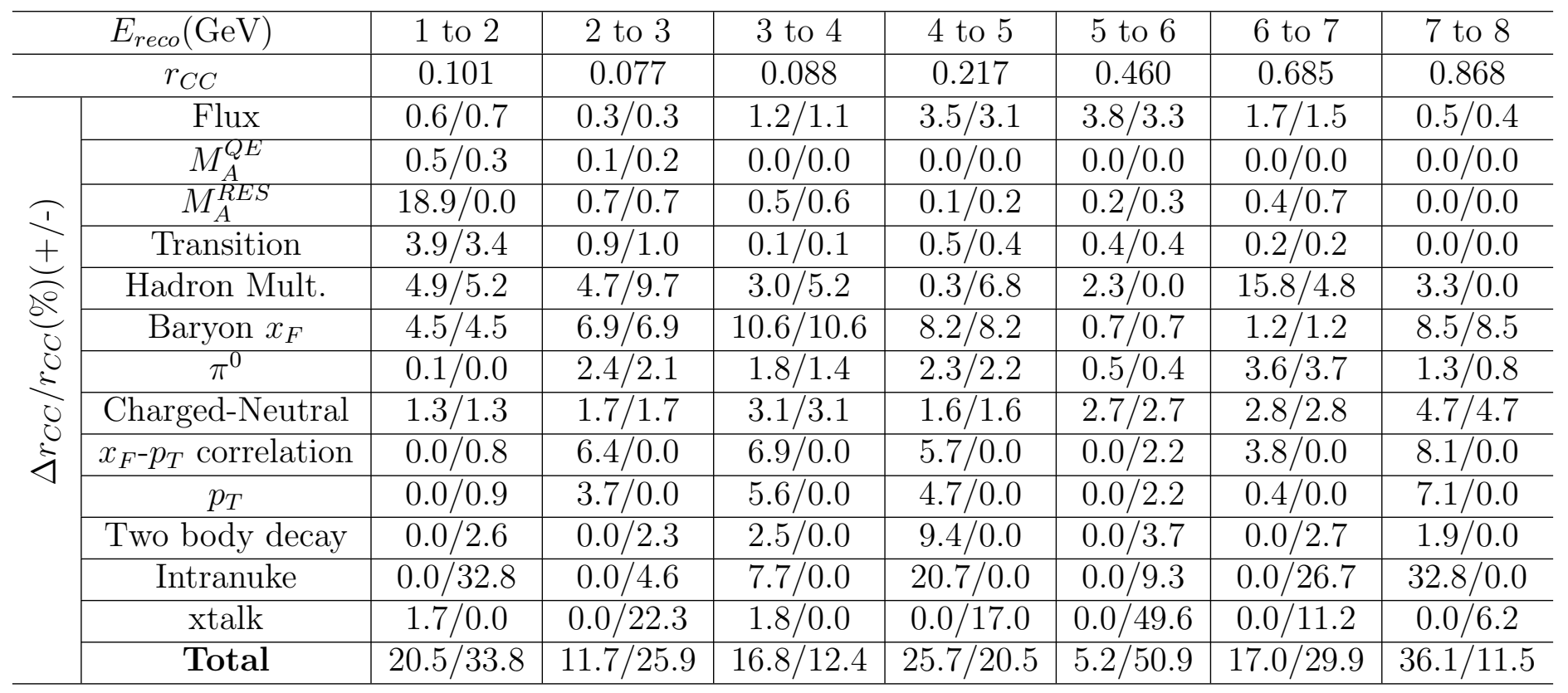

Table 7.3: Percentage systematic errors on $r_{C C}$ in reconstructed energy bins. 


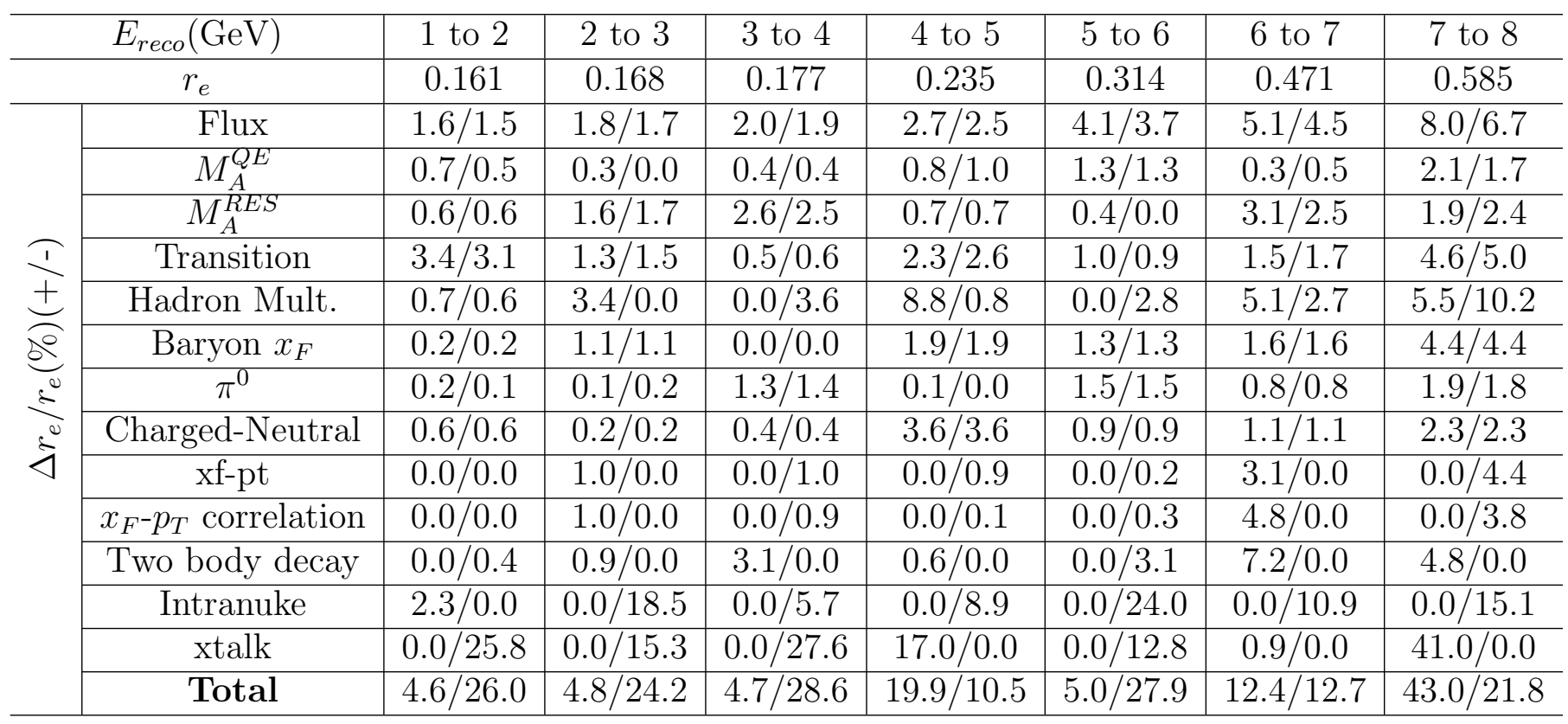

Table 7.4: Percentage systematic errors on $r_{e}$ in reconstructed energy bins.
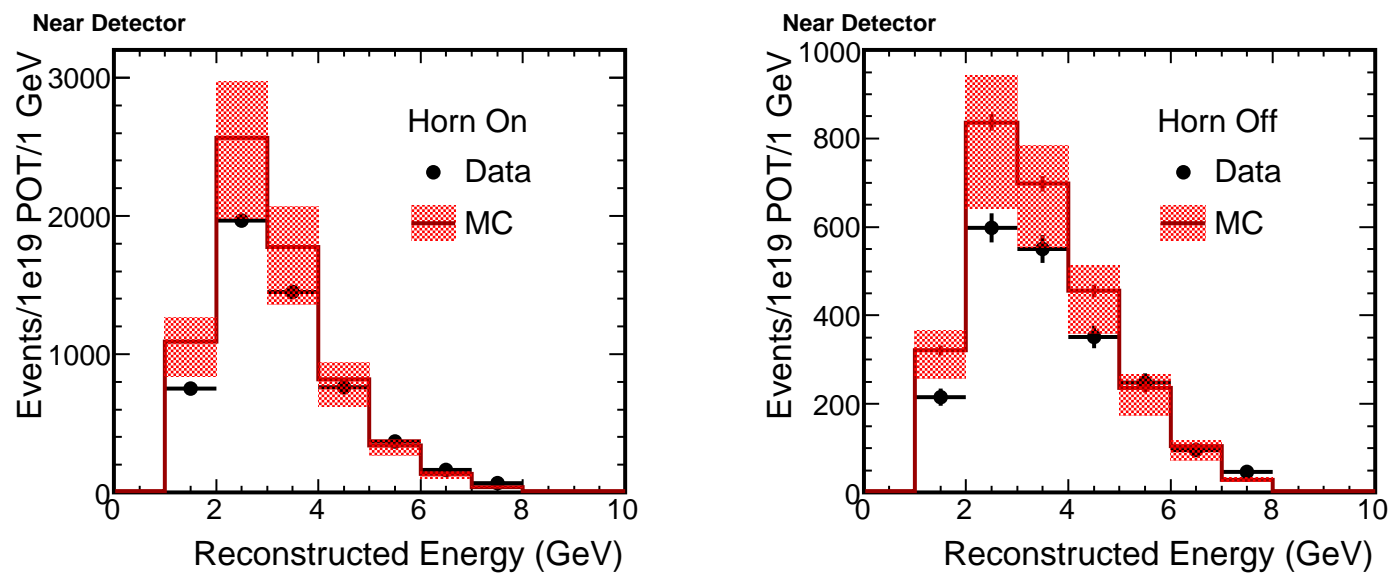

Figure 7.14: The reconstructed energy spectra of events that pass the $\nu_{e}$ selection algorithm. Left: horn-on; Right: horn-off. All spectra are normalized to $1 \times 10^{19}$ POTs. The error bar represents the statistical error while the error band represents the systematic error. 

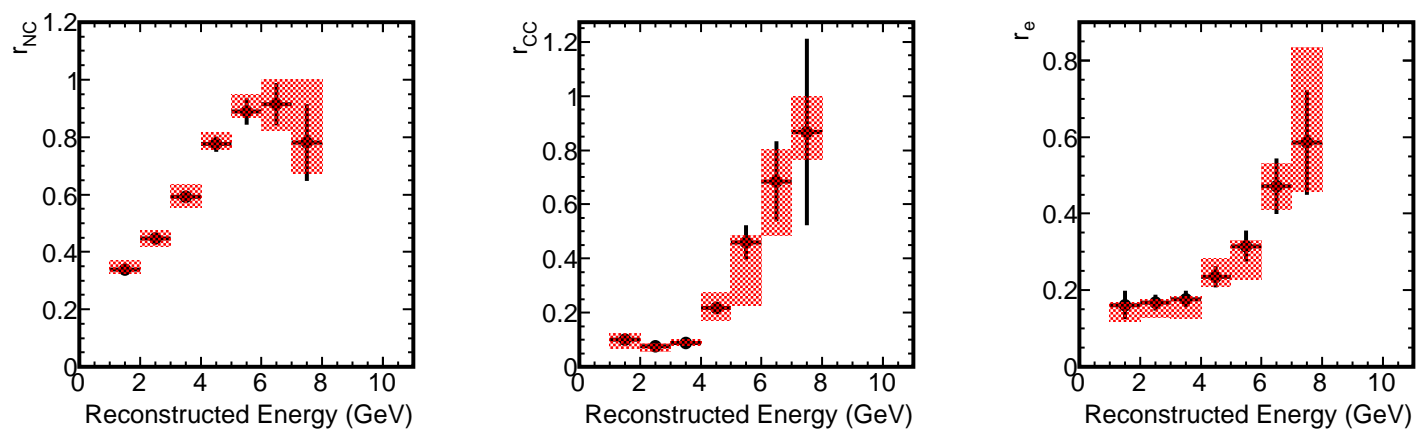

Figure 7.15: The ND horn-off over horn-on spectrum ratios. The error bars represent the MC statistics. The error bar represents the statistical error while the error band represents the systematic error.

beam $\nu_{e}$ contribution. The resulting spectra are shown in Fig.7.16. Since there cannot be negative events and the individual background components cannot exceed the observed data (ignoring the statistical uncertainty in the data), when deriving the NC and $\mathrm{CC}$ components, the solutions are required to fall within this region. Hence, if a negative result is obtained, instead of solving the equations, we simply scale down the $\mathrm{MC} \mathrm{NC}$ and $\mathrm{CC}$ components to match the data keeping the original $\mathrm{NC} / \mathrm{CC}$ ratio. Furthermore, if the estimated uncertainties extend beyond the allowed region (bounded by zero from below and by the data point from above) then the uncertainties are truncated to not extend beyond this range. Note this is only relevant for the highest energy bins. However at higher energies it also becomes less important to deconvolve the $\mathrm{NC}$ and $\mathrm{CC}$ contributions, since oscillations will have less effect on the extrapolation in this range.

The total NC and CC contributions are derived from the sum of the individual bin contributions. When combining bins, the statistical errors are added in quadrature, while the systematic errors associated with the horn off/on ratios are each added linearly taking into account the sign of deviation in each bin. The total uncertainty associated with each parameter is then added in quadrature to produce the final error 


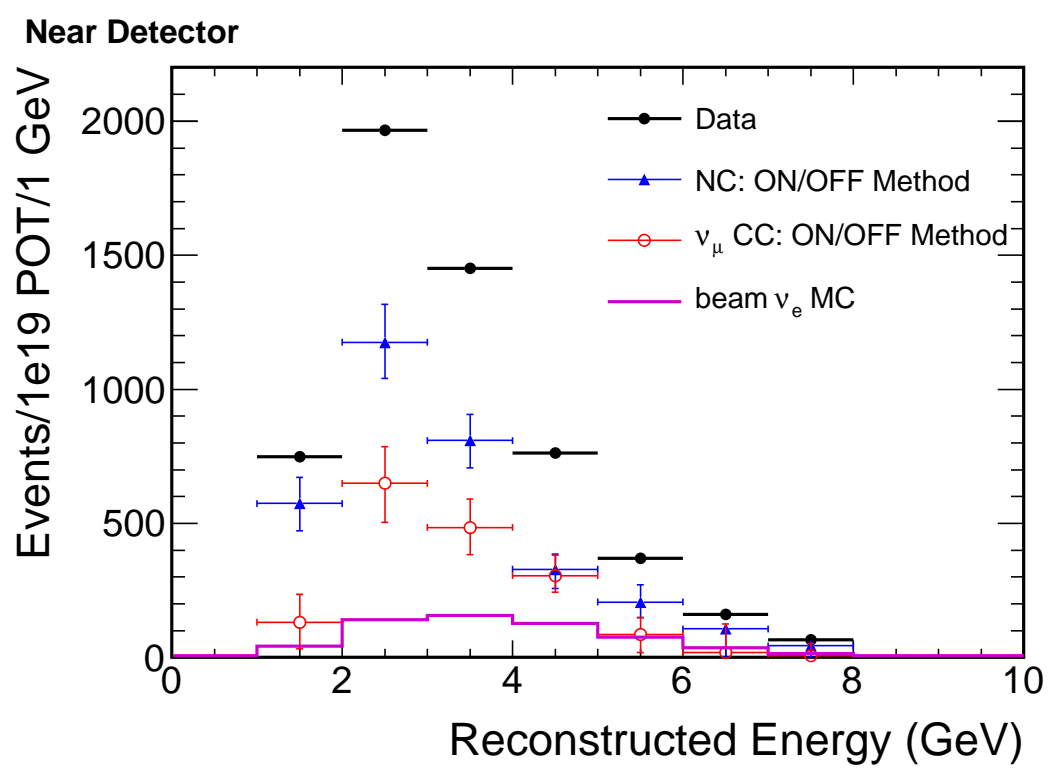

Figure 7.16: Reconstructed horn-on energy spectrum of events that pass the $\nu_{e}$ selection algorithm assuming $15 \%$ beam $\nu_{e}$ error.

on the background estimates. The resulting ND background components as well as a breakdown of the contributions to the $\mathrm{NC}$ and $\mathrm{CC}$ uncertainties are provided in Tables 7.5, 7.6, and 7.7. The uncertainty in the $\mathrm{NC}$ component is $9 \%$ and the uncertainty in the $\nu_{\mu} \mathrm{CC}$ component is $18 \%$.

\begin{tabular}{c|c|c|c|c}
\hline & Total & $N C$ & $C C$ & beam $\nu_{e}$ \\
\hline Data Driven: & $5524 \pm 35$ & $3246_{-291}^{+275}$ & $1685_{-281}^{+297}$ & $594 \pm 89$ \\
\hline Monte Carlo: & $6765 \pm 21$ & $4426 \pm 17$ & $1744 \pm 10$ & $594 \pm 6$ \\
\hline |MC-Data|/MC: & $18 \%$ & $27 \%$ & $3 \%$ & - \\
\hline
\end{tabular}

Table 7.5: The ND background estimates from the comparison of horn-off to horn-on data and the ND background estimates from the Monte Carlo prediction assuming $15 \%$ beam $\nu_{e}$ error. 


\begin{tabular}{c|c|c|c|c|c|c|c}
\hline & Total & $N^{\text {On Data }}$ & $N^{\text {Off Data }}$ & $N_{\text {beam } \nu_{e}}^{\text {On }}$ & $r_{N C}$ & $r_{C C}$ & $r_{e}$ \\
\hline Statistical: & ${ }_{-186}^{+168}$ & \pm 44 & \pm 194 & \pm 4 & \pm 96 & \pm 39 & \pm 29 \\
\hline Systematic: & ${ }_{-224}^{+218}$ & - & - & \pm 12 & \multicolumn{3}{|c}{${ }_{-224}^{+218}$} \\
\hline Stat. $\oplus$ Sys.: & \multicolumn{3}{|c|}{${ }_{-291}^{+275}$} \\
\hline
\end{tabular}

Table 7.6: Contributions to the ND background NC error assuming $15 \%$ beam $\nu_{e}$ error. Note total statistical errors are smaller than the individual statistical errors added in quadrature because of truncation applied to highest energy bin.

\begin{tabular}{c|c|c|c|c|c|c|c}
\hline & Total & $N^{\text {On Data }}$ & $N^{\text {Off Data }}$ & $N_{\text {beam } \nu_{e}}^{\text {On }}$ & $r_{N C}$ & $r_{C C}$ & $r_{e}$ \\
\hline Statistical: & ${ }_{-173}^{+192}$ & \pm 62 & \pm 194 & \pm 6 & \pm 96 & \pm 39 & \pm 29 \\
\hline Systematic: & ${ }_{-221}^{+227}$ & - & - & \pm 37 & \multicolumn{3}{c}{${ }_{-218}^{+224}$} \\
\hline Stat. $\oplus$ Sys.: & \multicolumn{8}{|c}{+297} \\
\hline
\end{tabular}

Table 7.7: Contributions to the ND background CC error assuming $15 \%$ beam $\nu_{e}$ error. Note total statistical errors are smaller than the individual statistical errors added in quadrature because of truncation applied to highest energy bin. 


\subsection{Summary}

In this chapter, we first described a method that can be used to obtain relative $\nu_{\mu} \mathrm{CC}$ and NC background contributions in the ND from comparison of background rates in the horn-on and horn-off configurations. We then used this method to calculate the $\mathrm{CC}$ and $\mathrm{NC}$ background components in the ND. The uncertainty in the NC component is $9 \%$ and the uncertainty in the $\nu_{\mu} \mathrm{CC}$ component is $18 \%$. Our next step is to extrapolate the ND spectra to the FD taking into account oscillations of different components to get the FD background rate prediction, which is the topic of the next chapter. 


\section{Chapter 8}

\section{Sensitivity to $\theta_{13}$}

In the previous chapter we described a data-driven method to obtain relative $\nu_{\mu} \mathrm{CC}$ and NC background contributions at the ND. In order to obtain the prediction of the number of $\nu_{e}$ selected background events at the FD, we extrapolate the backgrounds determined at the Near Detector to the Far Detector using the Far/Near ratios which are computed from Monte Carlo. A lot of systematic errors cancel to a large extent in the extrapolation. In this chapter, we will first describe the FD background prediction using the extrapolation method and discuss the systematic errors on the number of predicted background events and possible number of signal events expected at the Far Detector in this $\nu_{e}$ analysis. Then we will show the sensitivity of the MINOS experiment to the neutrino oscillation parameter $\theta_{13}$ based on an exposure of $3.25 \times$ $10^{20}$ protons on target.

\subsection{FD Background Prediction}

The FD $\nu_{e}$ background is composed of 4 neutrino interaction classes: $\mathrm{NC}, \nu_{\mu} \mathrm{CC}$, beam $\nu_{e} \mathrm{CC}$, and $\nu_{\tau} \mathrm{CC}$ which refer to the sample of each interaction type selected as a $\nu_{e}$ candidate by the ANN $\nu_{e}$ PID. We will rely on the Monte Carlo to estimate the 
numbers of beam $\nu_{e}$ and $\nu_{\tau}$ background events since they are reasonably well modeled by the Monte Carlo and/or their contributions are relatively small. For the NC and $\nu_{\mu} \mathrm{CC}$ backgrounds, we extrapolate the backgrounds determined at the Near Detector to the Far Detector using the Far/Near ratios. Following the outlined extrapolation procedure, the number of Far Detector background events in energy bin $i, N_{i}^{F}$, will be given by the equation:

$$
\begin{aligned}
N_{i}^{F} & =N_{N C, i}^{F}+N_{C C, i}^{F}+N_{b \nu_{e}, i}^{F}+N_{\nu_{\tau}, i}^{F} \\
& =\rho_{N C, i} N_{N C, i}^{N}+\rho_{C C, i} N_{C C, i}^{N}+N_{b \nu_{e}, i}^{F}+N_{\nu_{\tau}, i}^{F}
\end{aligned}
$$

where the Far/Near ratios

$$
\begin{aligned}
& \rho_{N C, i}=N_{N C, i}^{F} / N_{N C, i}^{N} \\
& \rho_{C C, i}=N_{C C, i}^{F} / N_{C C, i}^{N}
\end{aligned}
$$

are computed from the Monte Carlo. Fig.8.1 illustrates the extrapolation procedure for the NC and $\nu_{\mu} \mathrm{CC}$ background components. The ND spectra are obtained using the horn on/off background decomposition method described in the previous chapter. ND spectra are normalized to $1 \times 10^{19}$ POTs and FD spectra are normalized to $3.25 \times 10^{20}$ POTs. In the calculation of the $\rho$ values, we assume the following neutrino oscillation parameters:

$\theta_{12}=34^{\circ}, \theta_{23}=45^{\circ}, \theta_{13}=0, \Delta m_{21}=8.0 \times 10^{-5} \mathrm{eV}^{2},\left|\Delta m_{32}\right|=2.38 \times 10^{-3} \mathrm{eV}^{2}, \delta_{C P}=0$

We assume no $\nu_{\mu} \rightarrow \nu_{e}$ oscillation $\left(\theta_{13}=0\right)$ and no $\mathrm{CP}$ violation $\left(\delta_{C P}=0\right)$. We do not take into account the matter effects since they should only slightly affect the beam $\nu_{e}$ background which is a small contribution to the total background. The 
NC Far/Near ratio is relatively flat since the NC event rate is not affected by the neutrino oscillations. There is a clear increase of the $\nu_{\mu} \mathrm{CC}$ Far/Near ratio as energy goes up since the $\nu_{\mu} \rightarrow \nu_{\tau}$ oscillation is suppressed at high energies. Fig.8.2 shows the predicted FD background energy spectra for an exposure of $3.25 \times 10^{20}$ POTs. Table 8.1 summarizes the FD background prediction. We expect 27.4 background events for an exposure of $3.25 \times 10^{20}$ at the FD. The total MC number is scaled down by $20 \%$. This reflects the fact that MC does not reproduce data in the ND.
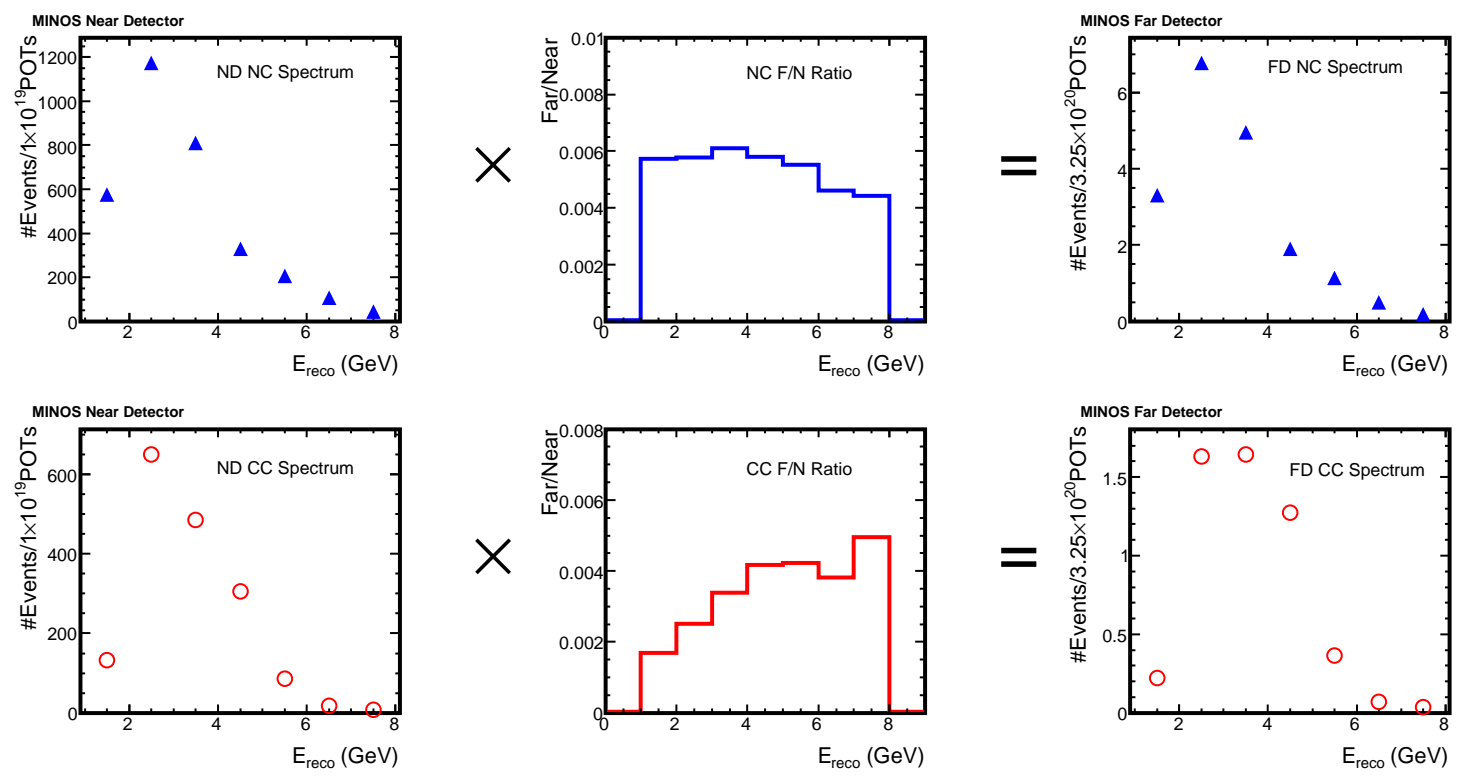

Figure 8.1: Extrapolation of $\mathrm{NC}$ and $\nu_{\mu} \mathrm{CC}$ background components from $\mathrm{ND}$ to $\mathrm{FD}$ taking into account oscillations. The ND spectra are obtained using the horn on/off background decomposition method. ND spectra are normalized to $1 \times 10^{19}$ POTs and FD spectra are normalized to $3.25 \times 10^{20}$ POTs.

\begin{tabular}{c|c|c|c|c|c}
\hline & Total & $\mathrm{NC}$ & $\nu_{\mu} \mathrm{CC}$ & beam $\nu_{e}(\mathrm{MC})$ & $\nu_{\tau}(\mathrm{MC})$ \\
\hline Monte Carlo & 34.32 & 25.71 & 5.18 & 2.33 & 1.10 \\
\hline Prediction & 27.43 & 18.76 & 5.24 & 2.33 & 1.10 \\
\hline
\end{tabular}

Table 8.1: FD background prediction $\left(3.25 \times 10^{20}\right.$ POTs $)$. 


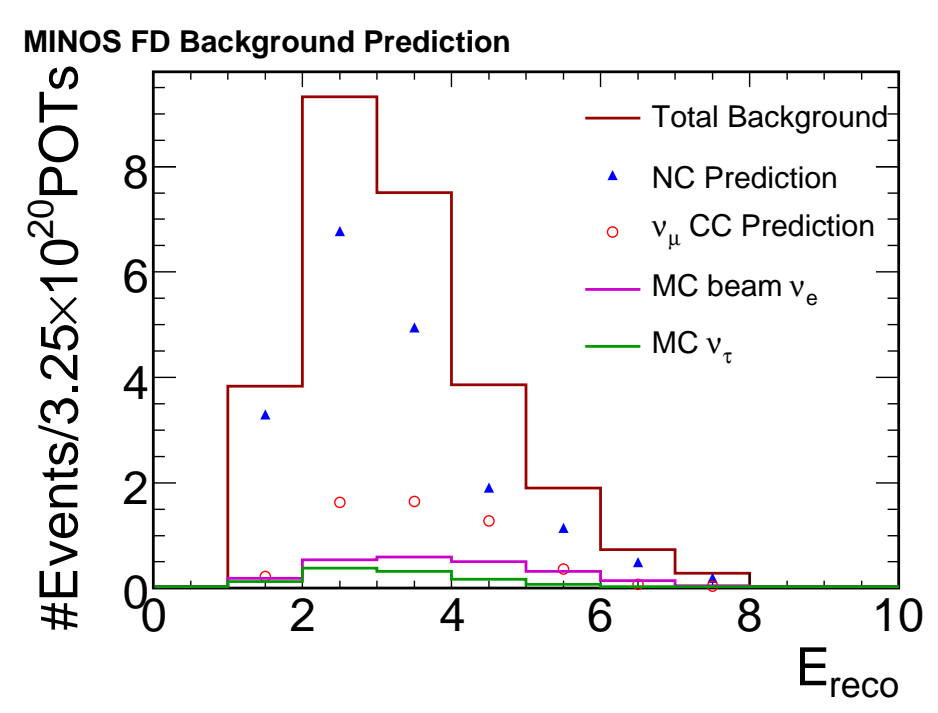

Figure 8.2: Predicted FD background energy spectra for an exposure of $3.25 \times 10^{20}$ POTs.

\subsection{Background Systematic Errors}

In this section, we discuss the systematic uncertainties in the predicted FD background events. The predicted $\mathrm{NC}$ and $\nu_{\mu} \mathrm{CC}$ background events are obtained from extrapolation. There are two sources of systematic errors: the ND data decomposition method (i.e. horn on/off method) and the extrapolation. The predicted beam $\nu_{e}$ and $\nu_{\tau}$ background events are obtained from Monte Carlo. The systematic errors for these two sources are the full uncertainties in the MC simulation.

\subsubsection{Uncertainties in NC and $\nu_{\mu}$ CC Predictions}

As we discussed in the previous chapter, the estimated $\mathrm{NC}$ background uncertainty is $9 \%$ and the estimated $\nu_{\mu} \mathrm{CC}$ background uncertainty is $18 \%$ at the ND. Since they are the dominant background components in the $\nu_{e}$ analysis, one could naively imagine the uncertainty in the prediction FD background events to be between $9 \%$ and $18 \%$. However, since these two background components are highly correlated (7.22), the 
error on the number of total background is significantly smaller than the errors on the individual components.

We make use of the fact that the sum of the components in the Near Detector must equal the total data sample

$$
N_{D a t a, i}^{N}=N_{N C, i}^{N}+N_{C C, i}^{N}+N_{b \nu_{e}, i}^{N}
$$

and rewrite the extrapolation equation for the $\mathrm{NC}$ and $\nu_{\mu} \mathrm{CC}$ backgrounds as

$$
\begin{aligned}
N_{N C+C C, i}^{F} & =\rho_{N C, i} N_{N C, i}^{N}+\rho_{C C, i} N_{C C, i}^{N} \\
& =\rho_{N C, i} N_{N C, i}^{N}+\rho_{C C, i}\left(N_{\text {Data }, i}^{N}-N_{N C, i}^{N}-N_{b \nu_{e}, i}^{N}\right) \\
& =\rho_{C C, i} N_{\text {Data }, i}^{N}+\left(\rho_{N C, i}-\rho_{C C, i}\right) N_{N C, i}^{N}-\rho_{C C, i} N_{b \nu_{e}, i}^{N}
\end{aligned}
$$

The error from ND data decomposition is

$$
\left(\delta N_{N C+C C, i}^{F}\right)_{D e c o m p}=\rho_{C C, i} \delta N_{D a t a, i}^{N}+\left(\rho_{N C, i}-\rho_{C C, i}\right) \delta N_{N C, i}^{N}-\rho_{C C, i} \delta N_{b \nu_{e}, i}^{N}
$$

$\delta N_{\text {Data, } i}^{N}$ is the statistical error on the total ND data, which is negligible. We also benefit from some cancellation in $\rho_{N C, i}-\rho_{C C, i}$. Different terms are added in quadrature in order to get the total error.

The ND data decomposition systematic errors are composed of statistical errors from horn-on/off data and MC and systematic errors from uncertainties in the hornoff/horn-on ratios and beam $\nu_{e}$ background. When we calculate the total error on the predicted FD background events, we add the statistical errors from the horn on/off method in quadrature between energy bins while the systematic errors are added linearly taking into account the sign of deviation in each energy bin. The result is

$$
\left.\left.\left(\delta N_{N C+C C}^{F}\right)_{\text {Decomp }}={ }_{-0.558}^{+0.555} \text { (stat. }\right) \oplus_{-0.668}^{+0.587} \text { (sys. }\right)=_{-0.87}^{+0.81} .
$$


Thus

$$
N_{N C+C C}^{F}=24.00_{-0.87_{\text {Decomp }}^{+0.81}}^{+}
$$

The systematic error from ND data decomposition is roughly $3.6 \%$ on the predicted $\mathrm{NC}$ and $\nu_{\mu} \mathrm{CC}$ background events.

Now we consider the systematic errors from extrapolation. Many people (in particular Josh Boehm of Harvard University) have worked on the evaluation of the systematic errors [146]. We will summarize the results here. The Far/Near ratios are calculated from Monte Carlo. The uncertainties discussed in the previous chapter when we calculated the systematic errors in the horn on/off method may also affect the Far/Near ratio. The uncertainties we have discussed are neutrino flux error, cross section error, hadronization error, intranuclear rescattering error, and the crosstalk modeling error. These systematic effects affect the modeling of MC Far/Near ratio but most of them should affect the two detectors in a similar manner so they should cancel to first order. There exist other effects which are more important for the modeling of Far/Near ratio since they could potentially give rise to Far/Near differences. These effects include uncertainties in the relative event rate normalization, absolute energy scale error, relative energy scale error, calibration errors, low pulse-height modeling error, and reconstruction. Now we describe each systematic effect in detail.

\section{Normalization}

The normalization error encompasses the differences between the two MINOS detectors due to uncertainty in the total exposure recorded in the two detectors. This includes uncertainties in the fiducial volume, or equivalently the fiducial mass of the detectors as well as uncertainty in the POT counting. Estimates for the size of this uncertainty are taken from the 2006 MINOS $\nu_{\mu}$ analysis. The terms included for this analysis are $1 \%$ from POT counting, $0.2 \%$ from steel thickness, $0.2 \%$ from uncertainty 
in the scintillator thickness, and an additional $2.1 \%$ from the uncertainty in the fiducial region (roughly 28 ton in the ND and $4.5 \mathrm{kton}$ in the FD). This gives a combined normalization error of $2.4 \%$.

\section{Absolute Energy Scale Uncertainty}

The goal of the MINOS calibration is to convert the raw detector response in ADC to absolute energy deposition in $\mathrm{GeV}$. An important intermediate step is the interdetector calibration, which is to convert the calibrated ADC value after removing variations within each detector to a universal energy unit - MIP, which is defined as the detector response to a $1 \mathrm{GeV}$ muon traversing 1 plane of scintillator at $90^{\circ}$ with respect to the longitudinal direction. The conversion factor is measured with the cosmic ray stopping muons and referred to as SigCorPerMip in the MINOS software. This conversion factor together with another factor which converts MIP to GeV determine the absolute energy scale. This parameter SigCorPerMip is used twice in the $\mathrm{MC}$ generation, first in the digitization to convert the true energy deposition to the detector response, then in the reconstruction to convert the detector response back to the universal energy unit MIP. The value of the absolute energy error is taken to be $5.7 \%$ from the calibration studies for the 2008 MINOS $\nu_{\mu} \mathrm{CC}$ analysis. In order to evaluate the uncertainties in the extrapolation, Monte Carlo samples are produced for the Near and Far detectors with the default SigCorPerMip factor shifted by the corresponding error for the reconstruction, but not in the digitization.

\section{Relative Energy Calibration Uncertainty}

Uncertainties in the relative energy calibration chain lead not only to differences in the reconstructed energy of events, but can also lead to changes in the pulse-height distribution in each event. To evaluate these uncertainties, we need to vary the calibration constants within their associated uncertainties. We produce Monte Carlo 
samples for the Near and Far detectors with the corresponding calibration constants modified for the reconstruction. Since we are interested in studying the effect of miscalibration, the digitization stage of the MC generation is still performed with the default calibration constants which results in a different set of calibration constants being applied at the digitization and reconstruction/calibration stages. We used this technique not only for this systematic but also for the other calibration uncertainties described in the next section. To evaluate the effects of the relative energy calibration uncertainties, we shift the SigCorPerMip factor for one detector and keep the factor for the other detector fixed. The resulting changes in the Far/Near ratios are added in quadrature. The Near Detector SigCorPerMip factor is shifted up and down by $2.3 \%$, and the Far Detector SigCorPerMip factor is shifted up and down by $2.4 \%$. Both numbers are taken from the MINOS $\nu_{\mu} \mathrm{CC}$ analysis.

\section{Other Calibration Uncertainties}

In order to evaluate the other calibration uncertainties, we produce Monte Carlo samples for the Near and Far detectors with the modified calibration constants. We take into account the following calibration effects:

1.PMT Gains Constants that convert the raw ADCs to photoelectrons. The gains are known to within a systematic shift of $\pm 5 \%$ and to within random channelto-channel variation of $7 \%$. The variation in PMT gains affects the $2 \mathrm{PE}$ cut we discussed in Chapter 6.

2.Linearity Correct for the PMT saturation at large pulse-heights. Errors are taken from the linear fits to the light injection data. The magnitude of this uncertainty is less than $1 \%$.

3.Strip-to-Strip Normalize the mean response of each strip to be equal. The mean variation in strip-to-strip response after calibration should be less than $0.5 \%$. 
4.Attenuation Normalizes the mean response along the position of each strip to be equal to the response in the middle of the strip. The residual variation along the strip after calibration is on the order of $1 \%$. At FD, we sum the readouts of two stripends which significantly reduces the attenuation effect.

\section{Low Pulse-height Modeling}

The low pulse-height depositions generated by the detector simulation have been shown to have poor agreement with data. While steps have been taken to reduce the dependence of the $\nu_{e}$ analysis on such low pulse-height hits, the cross talk model (which generates a large fraction of the low pulse-height simulated hits) and the removal of low pulse-height hits themselves need to be examined carefully to fully understand their impact on the $\nu_{e}$ background prediction.

All the $\nu_{e}$ discriminant variables are computed with hits whose pulse-height is greater than $2 \mathrm{PE}$, since hits below that cut are poorly modeled in MC simulation. This is done at the analysis level and thus the reconstruction remains unchanged. However, mis-modeling of the low pulse-height hits may affect the reconstruction and therefore affect the $\nu_{e}$ PID. To evaluate the reconstruction uncertainties associated with the mis-modeling of the low pulse height hits, we produce a special MC sample where we do the reconstruction with only hits above $2 \mathrm{PE}$. We compare the number of events accepted as $\nu_{e}$ candidates in the special MC sample with the number of accepted events in the standard sample and take the difference as the reconstruction systematic error associated with the pulse height cuts. We know that there are more hits less than 2PE in data (Chapter 6), thus the effect should be larger for data. However, as we will show in the summary table, the systematic error from low pulseheight mismodeling is relatively small, so we still use the error evaluated with MC. 


\section{Intensity Effects}

The very different event rates at the two detectors could alter the calculated Far/Near ratios. At our normal beam intensity (25E12 protons per spill), we expect on average 8 events in the Near Detector during the $10 \mu s$ spill time. However, we only expect to see on average 1 neutrino event per day in the Far Detector. At the Near Detector, we have to separate the overlaid neutrino events in each spill based on timing and spatial information. The events that occur late in the spill may be affected by the activities (PMT after-pulsing, neutrons etc.) caused by the earlier events. At the Far Detector, all the events are well separated in time. This can cause a potential Near/Far difference.

We compare the efficiency of selecting the first event in the spill with respect to the efficiency for all events at the ND. The first event in each spill should be unaffected by the other events in the same spill. Therefore the first event in each spill simulates the events at the FD and a comparison of the first event and all events can tell us the difference in the efficiencies of selecting $\nu_{e}$-like events at the two detectors. In order to get a clean sample, we only select the first event in each snarl if it is at least $100 \mathrm{~ns}$ away from the second event. Fig.8.3 shows the comparison of PID distributions for the first event in each spill and for all events. The distributions are quite consistent between the first event and all events for both data and MC. The difference in the relative efficiencies of selecting $\nu_{e}$-like events between data and $\mathrm{MC}$ is $1.6 \% \pm 1.9 \%$. This indicates that we are underestimating the FD background prediction by $1.6 \%$. Thus we will scale up the $\mathrm{NC}+\nu_{\mu} \mathrm{CC}$ background prediction by $1.6 \%$ and take the statistical error on that scale $(1.9 \%)$ as the systematic error associated the intensity effects. 

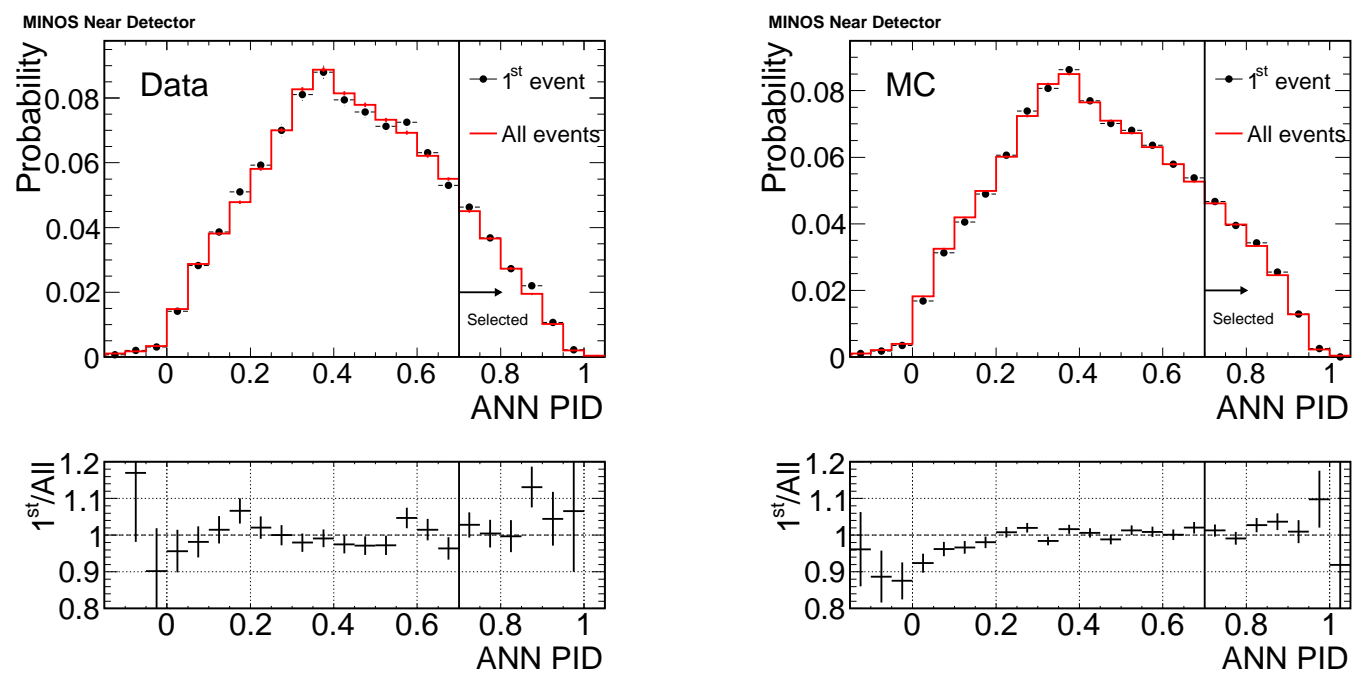

(a) Data

(b) $\mathrm{MC}$

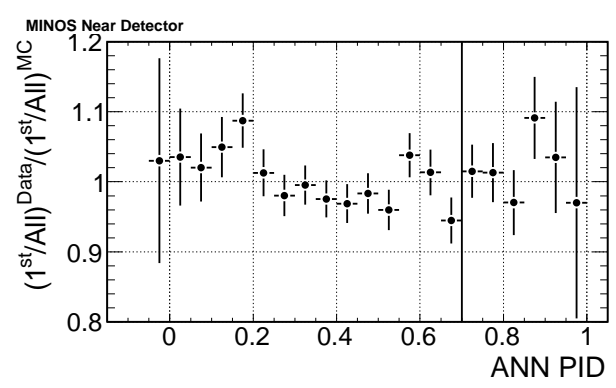

(c) Double ratio

Figure 8.3: Comparison of PID distributions for the first event in each spill (black points) and for all events (red curve). Distributions are area normalized. (a) Data; (b) MC; (c) Double ratio. 


\section{Detector Alignment}

Another effect we investigated is the detector alignment. The MINOS detectors consist of alternating planes of steel and scintillator strips mounted on the steel. The planes are not perfectly aligned during the detector installation. The actually location of each scintillator strip is measured using cosmic ray muon data. The measurements performed at both detectors indicate that the magnitude of detector misalignment is on the order of $6 \mathrm{~mm}$ for the ND and $3 \mathrm{~mm}$ for the FD. Since the Monte Carlo simulation assumes the scintillator strips are perfectly aligned, the misalignment could be one source of data/MC differences. Furthermore, if the misalignment does have a sizable effect on the $\nu_{e}$ selection efficiency, it could affect the Near and Far detectors differently since the magnitude of the misalignment is quite different for the two detectors.

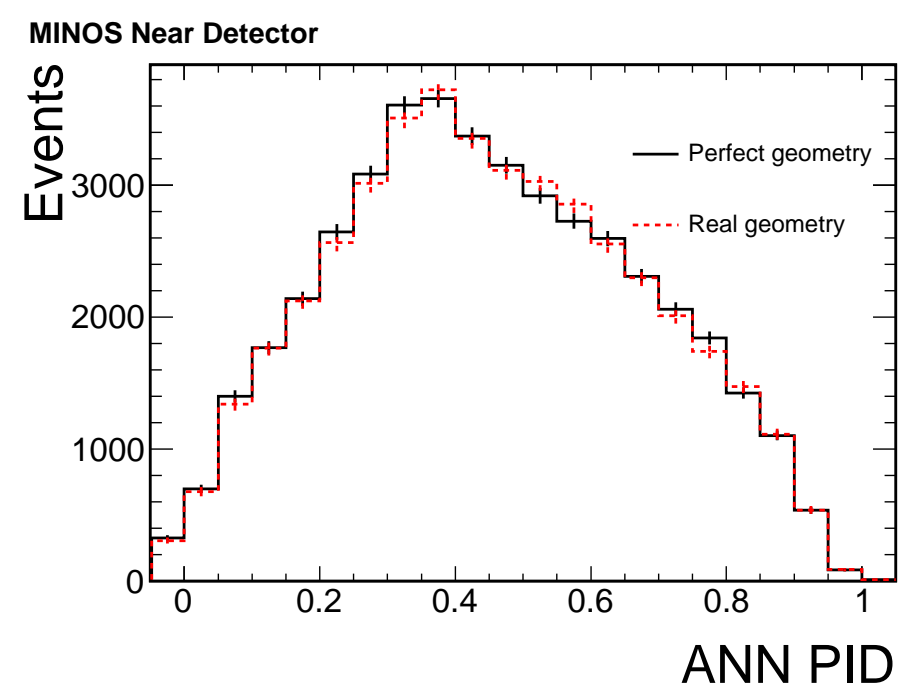

Figure 8.4: ANN PID distributions for MC with perfect geometry and with real geometry.

We have generated a small MC sample with the real strip locations measured from data (real geometry) and compared that with the standard MC (perfect geometry). 
The ANN PID distributions for those two samples are shown in Fig.8.4. The two distributions agree very well with statistics, which indicates the detector misalignment has a negligible effect on the $\nu_{e}$ analysis. Therefore we do not consider it in the evaluation of the systematics.

\section{Total NC+CC Systematic Errors}

We have described all the systematic effects we considered. Now we are going to evaluate their impacts on the FD NC and $\nu_{\mu} \mathrm{CC}$ background prediction. Since there are large bin to bin correlations, and the relevant quantity for the analysis is the uncertainty in the total number of events, we consider the total change in the number of $\mathrm{NC}$ and $\nu_{\mu} \mathrm{CC}$ events from the extrapolation uncertainties for each source of systematic explicitly as

$$
\left(\delta N_{N C+C C}^{F}\right)_{\text {Extrap }}=\sum_{i}\left(\delta \rho_{N C, i} N_{N C, i}^{N}+\delta \rho_{C C, i} N_{C C, i}^{N}\right)
$$

where we have defined $\left(\delta N_{N C+C C}^{F}\right)_{\text {Extrap }}$ as the uncertainty in the predicted NC and $\nu_{\mu}$ events from the extrapolation and $i$ denotes the $i^{\text {th }}$ energy bin. The justification of doing this is that each source of systematic affects $\mathrm{NC}$ and $\nu_{\mu} \mathrm{CC}$ background components in a correlated way. The effect from each systematic is then summed in quadrature to produce the total error on the summed number of predicted Far Detector $\mathrm{NC}$ and $\nu_{\mu} \mathrm{CC}$ events.

Table 8.2 summarizes the systematic errors from extrapolation for $\mathrm{NC}$ and $\nu_{\mu}$ CC background events selected by the ANN PID. Considering the total number of events, we obtain a systematic error on the order of $7 \%$ for $\mathrm{NC}$ and $13 \%$ for the $\nu_{\mu}$

CC components, and a total $(\mathrm{NC}+\mathrm{CC})$ of ${ }_{-6.0 \%}^{+6.5 \%}$. We also scale up the background prediction by $3.8 \%$ as to correct for the crosstalk mismodeling (discussed in section 
6.4) and intensity effect (discussed in section 8.2.1). Therefore we have:

$$
N_{N C+C C}^{F}=24.91_{-1.49_{\text {Extrap }}^{+1.62}}^{F}
$$

If we combine the error from extrapolation with the error from ND data decomposition, we have the predicted number of $\mathrm{NC}+\mathrm{CC}$ background events with errors:

$$
N_{N C+C C}^{F}=24.91 \pm 4.99(\text { stat. })_{-1.73}^{+1.81}(\text { sys. })
$$

\subsubsection{Uncertainties in the beam $\nu_{e}$ and $\nu_{\tau}$ backgrounds}

The beam $\nu_{e} \mathrm{CC}$ component of the spectrum is taken from the Monte Carlo; i.e., it is not extracted from ND. Therefore, the uncertainty in this event sample is the full uncertainty in the MC simulation. We have evaluated all the systematic effects described in the previous section on the beam $\nu_{e}$ background except the relative normalization and the relative energy scale since the Near/Far difference is irrelevant for the beam $\nu_{e}$ background estimation (we do not use extrapolation to predict this background source).

The efficiency of the $\nu_{e}$ selection has been determined using a data-driven technique with events identified as $\nu_{\mu}$ CC [147]. Tracks from identified muon events are removed and an electron with the momentum of the removed track is simulated in the MINOS detectors. The energy scale and event topology of electrons in our detectors are well simulated based on the comparison of the MINOS Calibration Detector data and MC. The MC electron energy depositions are merged with the remnant of the original $\nu_{\mu} \mathrm{CC}$ event and re-reconstructed to simulate a $\nu_{e} \mathrm{CC}$ interaction in the MINOS detectors. This technique is named MRE - Muon Removal with Electron addition. These merged events are then passed through the $\nu_{e}$ event selection algorithm. By making use of events that are selected in the manner in both data and 


\begin{tabular}{|c|c|c|c|c|c|c|}
\hline & \multicolumn{2}{|c|}{$\mathrm{NC}$} & \multicolumn{2}{|c|}{$\nu_{\mu} \mathrm{CC}$} & \multicolumn{2}{|c|}{ Total $(\mathrm{NC}+\mathrm{CC})$} \\
\hline Systematic & Down & Up & Down & $\mathrm{Up}$ & Down & Up \\
\hline Flux & $-0.6 \%$ & $0.7 \%$ & $-0.8 \%$ & $1.0 \%$ & $-0.6 \%$ & $0.8 \%$ \\
\hline$M_{A}^{Q E}$ & $-0.0 \%$ & $0.1 \%$ & $-0.0 \%$ & $0.0 \%$ & $-0.0 \%$ & $0.0 \%$ \\
\hline$M_{A}^{R E S}$ & $-0.0 \%$ & $0.0 \%$ & $-0.1 \%$ & $0.1 \%$ & $-0.0 \%$ & $0.0 \%$ \\
\hline Transition & $-0.2 \%$ & $0.2 \%$ & $-0.2 \%$ & $0.2 \%$ & $-0.1 \%$ & $0.1 \%$ \\
\hline Baryon $x_{F}$ & \multicolumn{2}{|c|}{$-2.7 \%$} & \multicolumn{2}{|c|}{$3.4 \%$} & \multicolumn{2}{|c|}{$-1.2 \%$} \\
\hline$\pi^{0}$ probability & $-0.3 \%$ & $0.3 \%$ & $-0.3 \%$ & $0.3 \%$ & $-0.1 \%$ & $0.1 \%$ \\
\hline Charged-Neutral correlation & \multicolumn{2}{|c|}{$-0.9 \%$} & \multicolumn{2}{|c|}{$1.4 \%$} & \multicolumn{2}{|c|}{$-0.3 \%$} \\
\hline$x_{F^{-}} p_{T}$ correlation & \multicolumn{2}{|c|}{$-1.1 \%$} & \multicolumn{2}{|c|}{$0.6 \%$} & \multicolumn{2}{|c|}{$-0.7 \%$} \\
\hline$p_{T}$ & \multicolumn{2}{|c|}{$-1.0 \%$} & \multicolumn{2}{|c|}{$0.8 \%$} & \multicolumn{2}{|c|}{$-0.6 \%$} \\
\hline Two body decay & \multicolumn{2}{|c|}{$-1.3 \%$} & \multicolumn{2}{|c|}{$0.7 \%$} & \multicolumn{2}{|c|}{$-0.8 \%$} \\
\hline Hadron Multiplicity & $-0.1 \%$ & $0.5 \%$ & $-0.7 \%$ & $2.5 \%$ & $-0.2 \%$ & $1.0 \%$ \\
\hline Intranuke & \multicolumn{2}{|c|}{$0.7 \%$} & \multicolumn{2}{|c|}{$0.4 \%$} & \multicolumn{2}{|c|}{$0.7 \%$} \\
\hline Absolute Energy Scale & $-0.7 \%$ & $1.7 \%$ & $-2.5 \%$ & $0.9 \%$ & $-1.1 \%$ & $1.5 \%$ \\
\hline Hadron Energy & $-0.3 \%$ & $1.1 \%$ & $-1.6 \%$ & $0.0 \%$ & $-0.6 \%$ & $0.7 \%$ \\
\hline Normalization & $-2.4 \%$ & $2.4 \%$ & $-2.4 \%$ & $2.4 \%$ & $-2.4 \%$ & $2.4 \%$ \\
\hline Relative Energy Scale(ND) & $-1.3 \%$ & $1.9 \%$ & $-3.9 \%$ & $2.7 \%$ & $-1.9 \%$ & $2.1 \%$ \\
\hline Relative Energy Scale(FD) & $-1.3 \%$ & $2.5 \%$ & $-0.6 \%$ & $4.0 \%$ & $-1.1 \%$ & $2.8 \%$ \\
\hline Gains(ND) & $-2.6 \%$ & $0.5 \%$ & $-3.3 \%$ & $8.8 \%$ & $-2.7 \%$ & $2.4 \%$ \\
\hline Gains(FD) & $-1.4 \%$ & $0.8 \%$ & $-2.4 \%$ & $1.4 \%$ & $-1.6 \%$ & $1.0 \%$ \\
\hline Attenuation & \multicolumn{2}{|c|}{$0.5 \%$} & \multicolumn{2}{|c|}{$-0.1 \%$} & \multicolumn{2}{|c|}{$0.4 \%$} \\
\hline Strip-to-Strip & \multicolumn{2}{|c|}{$-0.8 \%$} & \multicolumn{2}{|c|}{$-0.5 \%$} & \multicolumn{2}{|c|}{$-0.7 \%$} \\
\hline Linearity & \multicolumn{2}{|c|}{$0.5 \%$} & \multicolumn{2}{|c|}{$0.9 \%$} & \multicolumn{2}{|c|}{$0.6 \%$} \\
\hline Preselection & $-1.0 \%$ & $1.0 \%$ & $-1.0 \%$ & $1.0 \%$ & $-1.0 \%$ & $1.0 \%$ \\
\hline Low pulse-height modeling & \multicolumn{2}{|c|}{$1.1 \%$} & & & & \\
\hline Crosstalk modeling & 1.4 & & & & & \\
\hline Intensity effects & 1.9 & & & & & \\
\hline Total & $-6.3 \%$ & $6.5 \%$ & $-9.6 \%$ & $12.7 \%$ & $-6.0 \%$ & $6.5 \%$ \\
\hline
\end{tabular}

Table 8.2: Systematic errors from extrapolation on NC and $\nu_{\mu} \mathrm{CC}$ background events selected by ANN PID. 
$\mathrm{MC}$, the $\nu_{e} \mathrm{CC}$ selection efficiency can be measured. The difference in the efficiencies between data and $\mathrm{MC}$ is taken as the systematic error on the $\nu_{e}$ selection efficiency, which is on the order of $2.5 \%$.

The systematic errors on the beam $\nu_{e}$ background component in Near and Far Detector Monte Carlo are summarized in Table 8.3. The flux error is larger in the FD. The biggest uncertainty is due to the neutral kaon flux which is the least understood component of the NuMI beam. We have larger contribution of beam $\nu_{e}$ background from neutral kaon decay in the FD, therefore the flux error is large in the FD. The predicted number of FD beam $\nu_{e}$ background is:

$$
N_{b \nu_{e}}^{F}=2.33 \pm 1.53(\text { stat. })_{-0.39}^{+0.41}(\text { sys. })
$$

The $\nu_{\tau} \mathrm{CC}$ component of the spectrum is also taken from the Monte Carlo. Since this is a small background component, we assign a 50\% systematic error on this background which is dominated by the $\nu_{\tau}$ cross section uncertainty. The predicted number of $\nu_{\tau} \mathrm{CC}$ background is:

$$
N_{\nu_{\tau}}^{F}=1.10 \pm 1.05(\text { stat. })_{-0.55}^{+0.55}(\text { sys. })
$$

We combine all the background component to get the predicted FD background events:

$$
N_{\text {background }}^{F}=28.3 \pm 5.3(\text { stat. }) \pm 1.9(\text { sys. })
$$

The total systematic error on the background prediction is roughly $6.7 \%$. 


\begin{tabular}{|c|c|c|c|c|}
\hline & \multicolumn{2}{|c|}{ ND } & \multicolumn{2}{c|}{ FD } \\
\hline Systematic & Down & Up & Down & Up \\
\hline Flux & $-8.5 \%$ & $8.5 \%$ & $-12.5 \%$ & 12.5 \\
\hline$M_{A}^{Q E}$ & $-4.2 \%$ & $4.4 \%$ & $-4.2 \%$ & $4.3 \%$ \\
\hline$M_{A}^{R E S}$ & $-6.0 \%$ & $6.3 \%$ & $-6.1 \%$ & $6.4 \%$ \\
\hline Transition & $-5.9 \%$ & $5.9 \%$ & $-6.8 \%$ & $6.8 \%$ \\
\hline Baryon $x_{F}$ & \multicolumn{2}{|c|}{$-2.7 \%$} & \multicolumn{2}{|c|}{$-2.7 \%$} \\
\hline$\pi^{0}$ probability & $-0.8 \%$ & $0.8 \%$ & $-0.8 \%$ & $0.8 \%$ \\
\hline Charged-Neutral correlation & \multicolumn{2}{|c|}{$-1.5 \%$} & \multicolumn{2}{|c|}{$0.3 \%$} \\
\hline$x_{F}-p_{T}$ correlation & \multicolumn{2}{|c|}{$-0.8 \%$} & \multicolumn{2}{|c|}{$-1.1 \%$} \\
\hline$p_{T}$ & \multicolumn{2}{|c|}{$-0.7 \%$} & \multicolumn{2}{|c|}{$-0.8 \%$} \\
\hline Two body decay & \multicolumn{2}{|c|}{$-1.1 \%$} & \multicolumn{2}{|c|}{$-0.4 \%$} \\
\hline Hadron Multiplicity & $-1.9 \%$ & $0.0 \%$ & $-1.1 \%$ & $0.0 \%$ \\
\hline Intranuke & \multicolumn{2}{|c|}{$1.1 \%$} & \multicolumn{2}{|c|}{$-0.5 \%$} \\
\hline Absolute Energy Scale & $-6.4 \%$ & $2.3 \%$ & $-1.0 \%$ & $2.2 \%$ \\
\hline Hadron Energy & $-2.3 \%$ & $3.8 \%$ & $-2.6 \%$ & $4.7 \%$ \\
\hline Gains & $-3.3 \%$ & $3.4 \%$ & $-2.0 \%$ & $0.2 \%$ \\
\hline Attenuation & \multicolumn{2}{|c|}{$0.8 \%$} & \multicolumn{2}{|c|}{$0.0 \%$} \\
\hline Strip-to-Strip & \multicolumn{2}{|c|}{$-0.6 \%$} & \multicolumn{2}{|c|}{$0.4 \%$} \\
\hline Linearity & \multicolumn{2}{|c|}{$-0.2 \%$} & $-0.3 \%$ \\
\hline Tow pulse-height modeling & \multicolumn{2}{|c|}{$-0.4 \%$} & \multicolumn{2}{|c|}{$-0.7 \%$} \\
\hline Crosstalk modeling & \multicolumn{2}{|c|}{$0.4 \%$} & \multicolumn{2}{|c|}{$0.5 \%$} \\
\hline Efficiency (MRE) & $-2.0 \%$ & $2.4 \%$ & $-2.0 \%$ & $2.4 \%$ \\
\hline \hline Total & $-15.5 \%$ & $14.8 \%$ & $-16.9 \%$ & $17.5 \%$ \\
\hline
\end{tabular}

Table 8.3: Systematic errors on the beam $\nu_{e}$ background events selected by ANN PID in Near and Far Detector Monte Carlo. 


\subsection{Signal Systematic Errors}

The predicted numbers of $\mathrm{NC}$ and $\nu_{\mu} \mathrm{CC}$ background events are directly extrapolated from a measurement of the equivalent samples in the MINOS Near Detector. However, the potential signal $\nu_{e}$ events from $\nu_{\mu} \rightarrow \nu_{e}$ oscillation have no Near Detector equivalent. In addition to the systematics discussed previously, the signal $\nu_{e}$ errors have contributions arising from the additional steps necessary to derive their flux at the Far Detector. The flux of signal $\nu_{e}$ events is predicted using the spectrum of $\nu_{\mu}$ $\mathrm{CC}$ selected events. This is equivalent to a Far/Near approach following the $\nu_{\mu} \mathrm{CC}$ disappearance analysis [59], the dominant systematics that are relevant to that result are a $4 \%$ normalization systematic, a $50 \%$ NC scale uncertainty and a $10 \%$ error on the $\nu_{\mu}$ CC energy scale. That predicted spectrum is then multiplied by the efficiency for selecting signal $\nu_{e}$ events at the Far Detector calculated using Monte Carlo. Since the Monte Carlo is tuned to match the $\nu_{\mu} \mathrm{CC}$ spectrum, the result obtained with this method is close to the prediction using FD Monte Carlo by construction. This reflects the fact that we understand the neutrino flux and $\nu_{e}$ selection efficiency quite well. The uncertainty in the efficiency of the $\nu_{e}$ selection is determined using the MRE (Muon Removal and Electron addition) technique described previously.

Table 8.4 summarizes the systematic errors on the signal $\nu_{e}$ events selected by ANN. The total systematic error is estimated to be $7.3 \%$. The major contributors to this error are the normalization and CC shower energy scale arriving from the normalization of this sample to the $\nu_{\mu} \mathrm{CC}$ events measured in the ND.

If $\theta_{13}$ is at CHOOZ limit $\left(\sin ^{2} 2 \theta_{13}=0.15\right)$ and we ignore the matter effects while the other oscillation parameters are the same as in 8.5, the predicted number of signal events is

$$
N_{\text {signal }}^{F}=10.7 \pm 3.3(\text { stat. }) \pm 0.8(\text { sys. }) \text { No matter effects }
$$

If we take into account matter effects, the predicted numbers of signal events for 
normal mass hierarchy $\left(\Delta m_{32}^{2}>0\right)$ and inverted mass hierarchy $\left(\Delta m_{32}^{2}<0\right)$ are

$$
\begin{aligned}
& N_{\text {signal }}^{F}=11.6 \pm 3.4(\text { stat. }) \pm 0.8(\text { sys. }) \text { (Normal hierarchy) } \\
& N_{\text {signal }}^{F}=7.0 \pm 2.6(\text { stat. }) \pm 0.5(\text { sys. }) \quad(\text { Inverted hierarchy })
\end{aligned}
$$

\subsection{MINOS Sensitivity to $\theta_{13}$}

We have worked out the predicted number of background events at the FD and the systematic error on the prediction. Using this information we can calculate the sensitivities for the $\nu_{e}$ analysis.

In our case the size of the data sample is small. We can treat the sample size as a Poisson-distributed variable. If we expect $N_{E x p}$ events, the probability to observe $N_{O b s}$ events can be written as $f\left(N_{O b s} ; N_{E x p}\right)$, where $f$ is the Poisson function. We define the $\chi^{2}$ metric as logarithm of the likelihood ratio $\lambda=f\left(N_{O b s} ; N_{E x p}\right) / f\left(N_{O b s} ; N_{O b s}\right)$ times $-2[32]$ :

$$
\chi_{0}^{2}=-2 \ln \lambda=2\left(N_{E x p}-N_{O b s}+N_{O b s} \ln \frac{N_{O b s}}{N_{E x p}}\right)
$$

This value does account for the statistical errors associated with a measurement, but neglects systematic errors. The $\chi^{2}$ is therefore rescaled as follows if we take into account the systematic errors [148]:

$$
\chi^{2}=\chi_{0}^{2} \times \frac{N_{E x p}}{\sigma_{\text {background }}^{2}+\sigma_{\text {signal }}^{2}+N_{\text {Exp }}},
$$

where $\sigma_{\text {background }}$ and $\sigma_{\text {signal }}$ represent the systematic errors on the predicted number of background and signal events.

When performing a fit $N_{O b s}$ would be the actual number of observed FD data 


\begin{tabular}{|c|c|c|}
\hline Systematic & Down & $\mathrm{Up}$ \\
\hline Flux & $-1.1 \%$ & $1.3 \%$ \\
\hline$M_{A}^{Q E}$ & $-0.3 \%$ & $0.3 \%$ \\
\hline$M_{A}^{R E S}$ & $-0.0 \%$ & $0.1 \%$ \\
\hline Transition & $-0.1 \%$ & $0.0 \%$ \\
\hline Baryon $x_{F}$ & \multicolumn{2}{|c|}{$-2.2 \%$} \\
\hline$\pi^{0}$ probability & $-0.7 \%$ & $0.7 \%$ \\
\hline Charged-Neutral correlation & \multicolumn{2}{|c|}{$-1.4 \%$} \\
\hline$x_{F-} p_{T}$ correlation & \multicolumn{2}{|c|}{$-0.7 \%$} \\
\hline$p_{T}$ & \multicolumn{2}{|c|}{$-0.5 \%$} \\
\hline Two body decay & \multicolumn{2}{|c|}{$-0.5 \%$} \\
\hline Hadron Multiplicity & $-1.4 \%$ & 1.7 \\
\hline Intranuke & \multicolumn{2}{|c|}{$-1.0 \%$} \\
\hline Absolute Energy Scale & $-1.7 \%$ & $1.8 \%$ \\
\hline Normalization & $-4.0 \%$ & $4.0 \%$ \\
\hline Gains & $-0.6 \%$ & $0.4 \%$ \\
\hline Attenuation & \multicolumn{2}{|c|}{$0.0 \%$} \\
\hline Strip-to-Strip & \multicolumn{2}{|c|}{$0.0 \%$} \\
\hline Linearity & \multicolumn{2}{|c|}{$0.0 \%$} \\
\hline Preselection & $-1.0 \%$ & $1.0 \%$ \\
\hline Low pulse-height modeling & \multicolumn{2}{|c|}{$0.9 \%$} \\
\hline Crosstalk modeling & \multicolumn{2}{|c|}{$0.1 \%$} \\
\hline NC Scale & $-0.9 \%$ & $1.0 \%$ \\
\hline CC Shower Energy & $-3.6 \%$ & $3.4 \%$ \\
\hline Efficiency (MRE) & $-2.0 \%$ & $2.4 \%$ \\
\hline Total & $-7.2 \%$ & $7.3 \%$ \\
\hline
\end{tabular}

Table 8.4: Systematic errors on the signal $\nu_{e}$ events selected by ANN. 
events, and $N_{E x p}$ would be the prediction from a particular set of oscillation conditions. The $\chi^{2}$ value would then be minimized in order to find the best fit point oscillation values. The goal of the sensitivity is to present the limit that MINOS could set if the MINOS were to measure the null hypothesis $\left(U_{e 3}=0\right)$. Therefore we fix $N_{O b s}$ to the number of events predicted for $U_{e 3}=0$ case and $N_{E x p}$ is allowed to vary by changing the input oscillation parameters. We fix $\Delta m_{32}^{2}$ and $\theta_{23}$ to the best fit values of MINOS $\nu_{\mu} \mathrm{CC}$ disappearance measurement: $\left|\Delta m_{32}^{2}\right|=0.0024 \mathrm{eV}^{2}$ and $\sin ^{2} 2 \theta_{23}=1$. A search for $\chi^{2}$ minimum is performed over a 2-D grid of points in $\sin ^{2}\left(2 \theta_{13}\right)$ and $\delta_{C P}$. In addition the oscillations are calculated under the assumption of the normal and inverted mass hierarchies. At each point, the number of events that would be observed for those oscillation probabilities is calculated, this is used to generate a $\chi^{2}$ value. The sensitivities of interest are therefore the true parameters that are excluded at $90 \%$ confidence level when we observe a number of events consistent with $U_{e 3}=0$. This contour is defined as a cut at $\chi^{2}=2.71$. Note we are not trying to measure or constrain $\delta_{C P}$, so we show our limit on $\theta_{13}$ as a function of $\delta_{C P}$. The number of degrees of freedom is 1 rather than 2 .

Fig.8.5 shows the $\chi^{2}$ distributions as a function of $\sin ^{2}\left(2 \theta_{13}\right)$ and $\delta_{C P}$ for normal (left) and inverted (right) mass hierarchies.

Fig.8.6 shows the $1-\mathrm{d} \chi^{2}$ distributions if we assume $\delta_{C P}=0$. The matter effects have a large impact on the $\nu_{\mu} \rightarrow \nu_{e}$ oscillation as we discussed in Chapter 3. For the normal (inverted) hierarchy, matter effects enhance (suppress) the transition probabilities for neutrinos. Therefore the limit we could set on $\sin ^{2}\left(2 \theta_{13}\right)$ is quite different for the two mass hierarchies.

Fig.8.7 shows the MINOS sensitivity to the neutrino mixing angle $\theta_{13}$ as a function of $\delta_{C P}$ at $3.25 \times 10^{20}$ POTs. A comparison to the CHOOZ limit indicates that the MINOS experiment even with this initial data set has the potential to explore the region not excluded by the $\mathrm{CHOOZ}$ experiment. 

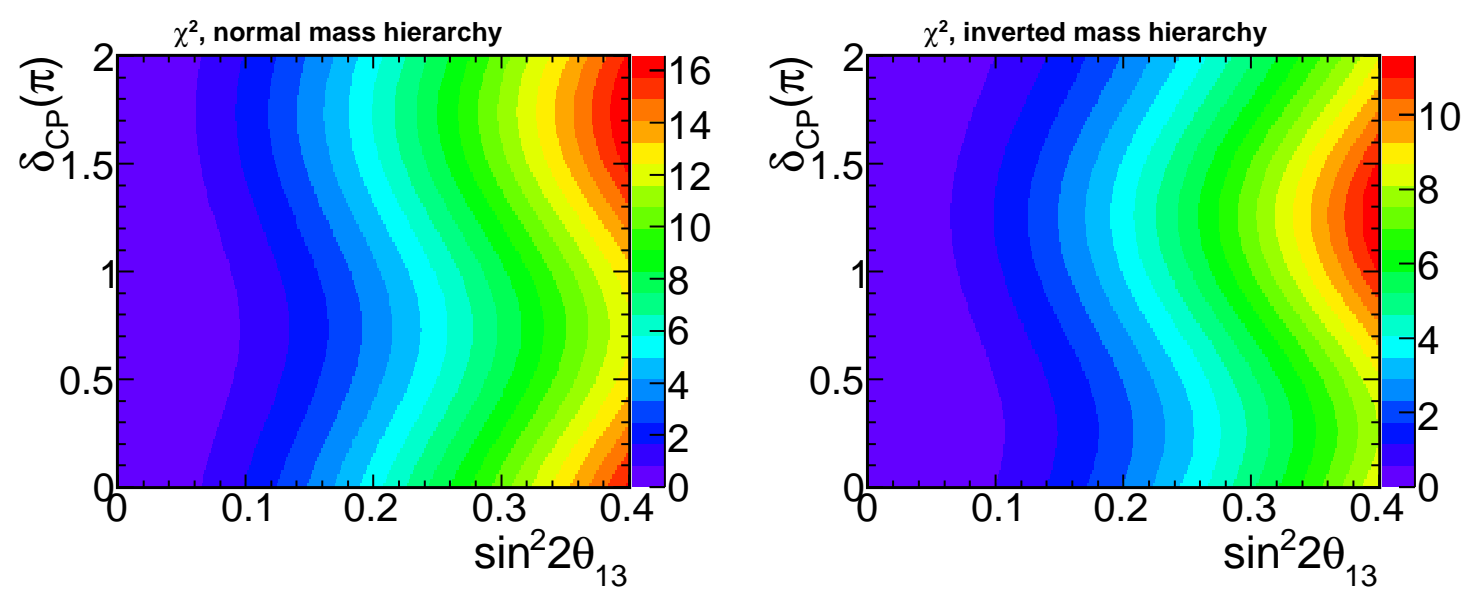

Figure 8.5: $\chi^{2}$ distributions as a function of $\sin ^{2}\left(2 \theta_{13}\right)$ and $\delta_{C P}$. We assume $\left|\Delta m_{32}^{2}\right|=$ $0.0024 \mathrm{eV}^{2}$ and $\sin ^{2} 2 \theta_{23}=1$. The exposure is taken to be $3.25 \times 10^{20}$ POTs. Left: normal mass hierarchy $\left(\Delta m_{32}^{2}>0\right)$; Right: inverted mass hierarchy $\left(\Delta m_{32}^{2}<0\right)$.

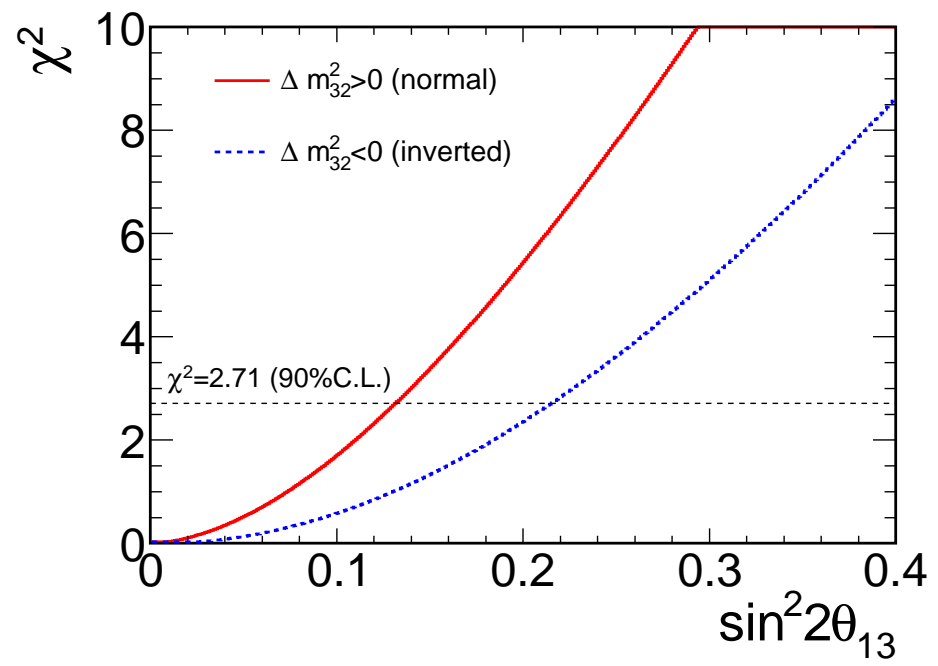

Figure 8.6: $\chi^{2}$ distributions as a function of $\sin ^{2}\left(2 \theta_{13}\right)$. We assume $\delta_{C P}=0,\left|\Delta m_{32}^{2}\right|=$ $0.0024 \mathrm{eV}^{2}$ and $\sin ^{2} 2 \theta_{23}=1$. The exposure is taken to be $3.25 \times 10^{20}$ POTs. 


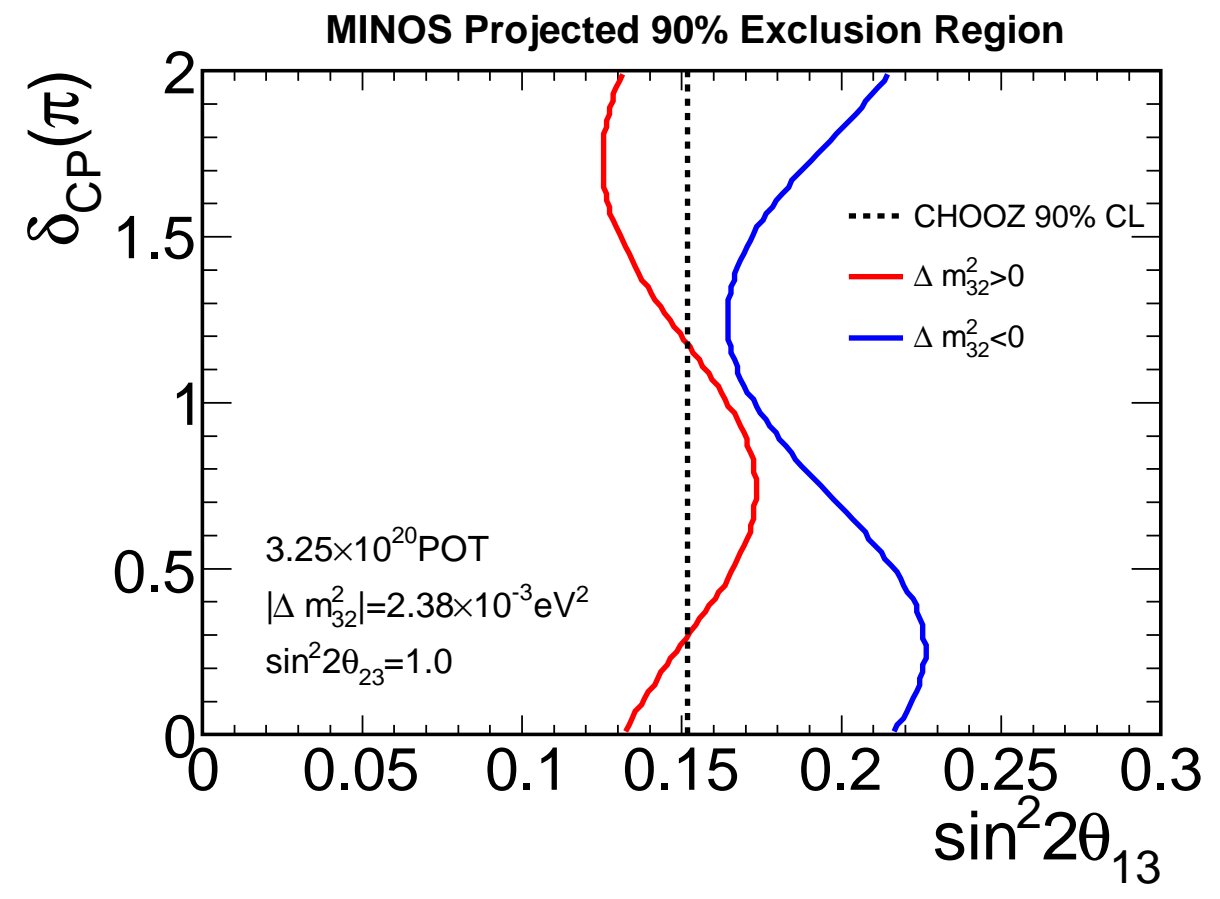

Figure 8.7: MINOS sensitivity as a function of $\delta_{C P}$ at $3.25 \times 10^{20}$ POTs. 


\section{Chapter 9}

\section{First Results of MINOS $\nu_{e}$ Analysis}

\subsection{Blind Analysis and Sidebands}

In order to eliminate the biases that may affect the physics analysis, the first MINOS $\nu_{e}$ analysis was done via blind analysis. The procedures adopted for the blind analysis are outlined as follows. All of the Near Detector data and out-of-spill Far Detector data (events not coincident with the NuMI spills) are open. The ND data are used to study neutrino flux and cross section, perform detector calibration, decide on selection cuts and analysis, and measure the background in the $\nu_{e}$ analysis. The FD out-of-spill data are used to perform the detector calibration, study the detector response and measure the cosmic ray background in the $\nu_{e}$ analysis. The entire FD in-spill data (events coincident with the NuMI spills) remain hidden from the analysis group (and the whole collaboration) until the event selection algorithm is finalized and the full systematic effects are understood at a satisfactory level.

Before we looked at the FD data in the signal region, we investigated three sidebands. The first sideband is called the anti-PID sideband, consisting of events with an ANN PID less than 0.55. This cut value was optimized to give a sufficiently large 
sample for analysis and minimize the possible signal to background ratio if oscillations do occur with $\sin ^{2} 2 \theta_{13}$ near the CHOOZ limit. This sideband was primarily used to check the normalization of the background prediction. The second sideband consists of events with an ANN PID between 0.55 and 0.7. This sideband is right outside the signal region. The third sideband is called the MRCC (muon removed charged-current showers) sideband, consisting of $\nu_{\mu} \mathrm{CC}$ events where the muon track is removed and the reconstruction is redone on the residual hits. This sample provides an excellent tool to study the hadronic showers. By construction, this sideband is signal-free and was used to check the corrections to background acceptance based on ND data. It was determined that before moving on to look at the data in the signal region ("open box"), the number of events selected for each sideband sample in the FD data must match the prediction of the number of events to within $2 \sigma$.

The MINOS $\nu_{e}$ analysis group opened the box on January 27, 2009 after satisfactory results were obtained for the three sidebands. In this chapter, we will summarize the first results from the MINOS $\nu_{e}$ appearance analysis. This chapter is organized as follows. We will first describe how we select Far Detector Data. Then we will show data and MC comparisons as we make successive cuts, namely, fiducial volume cuts, $\nu_{e}$ pre-selection cuts, and ANN PID cut. In the end, we will show our fit results.

\subsection{Far Detector Data}

In this first MINOS $\nu_{e}$ appearance analysis, we use the Far Detector data taken between May 2005 and July 2007 (MINOS Run I and Run II periods). All Far Detector runs passing a simple pre-selection on run type (physics or modified physics), run duration ( $>2$ minutes), and number of triggers ( $>100$ spills) have been reconstructed. Only data taken in the low energy (LE) configuration are considered in this analysis. The following data selection criteria are then applied to the data: 
- Data Quality Cuts: Only far detector data that have been collected with a full detector readout and with all detector systems operating normally are considered in the analysis. The following data quality cuts are applied to the data:

- The high voltage in each super-module must be operating normally.

- The magnetic coil in each super-module must be operating normally.

- The timing system must be operating normally, with a recorded GPS error of $<1 \mu s$.

- All 16 electronics crates must be active in the far detector readout.

- Beam Quality Cuts: The following beam quantities must be in the normal ranges: protons on target per spill, horn current, X-position of beam at target, Y-position of beam at target, horizontal width of beam at last profile monitor before target, and vertical width of beam at last profile monitor before target.

- Spill Timing Cuts: Events are required to be coincident with a recorded beam spill. The relative time between the earliest hit in an event and the predicted spill time at the Far Detector is required to lie in the range: $-2 \mu s<$ event time - spill time $<+12 \mu$ s.

- Light Injection Cuts: Events in time with Light Injection trigger are removed from the analysis. Light Injection events are additionally removed using a LI pattern recognition algorithm.

- Cosmic Muon Rejection: In the Far Detector, the rate of cosmic muons coincident with beam spills is comparable to the rate of neutrino interactions. The majority of cosmic muons enter the Far Detector at steep angles to the beam direction. To reduce the background from cosmic muons, we remove events that have large reconstructed angles with respect to the beam direction. 
Only about $3 \%$ of the total spills are eliminated by these cuts. The total exposure to the NuMI neutrino beam after the data and beam quality cuts is $3.14 \times 10^{20}$ protons on target (POTs). In the next sections we will examine various distributions at different $\nu_{e}$ selection cut levels. All the distributions are POT normalized (absolute normalization). Generally the standard MC is used to calculate the predictions. But for the PID distribution and reconstructed energy spectra of accepted events, we correct the standard MC predictions based on the data/MC differences measured at the Near Detector, i.e. the number of predicted background events is determined using the full ND data decomposition (horn on/off method described in Chapter 7) and $\mathrm{F} / \mathrm{N}$ extrapolation (described in Chapter 8). In all subsequent plots, the $\mathrm{MC}$ predictions are calculated using the following oscillation parameters:

$\theta_{13}=0, \delta_{C P}=0, \theta_{23}=45^{\circ}, \theta_{12}=34^{\circ},\left|\Delta m_{32}\right|=2.38 \times 10^{-3} \mathrm{eV}^{2}, \Delta m_{21}=8.0 \times 10^{-5} \mathrm{eV}^{2}$.

Generally assumption is made of no signal unless stated to the contrary when CHOOZ limit is used. We do not take into account the matter effects except for the final fitting results.

\subsection{Fiducial Volume Cuts}

The Far Detector fiducial volume is defined as

$$
0.5 \leq \sqrt{x^{2}+y^{2}} \leq \sqrt{14} \mathrm{~m}, 0.48 \leq z \leq 14.28 \mathrm{~m} \text { or } 16.26 \leq z \leq 27.97 \mathrm{~m}
$$

where $(\mathrm{x}, \mathrm{y}, \mathrm{z})$ are the coordinates of the reconstructed event vertex, and $\mathrm{z}$ axis is along the beam direction. The lower radial cut is to exclude the coil hole. The two intervals of $z$ cut correspond to the two super-modules of FD.

Fig.9.1 shows the reconstructed vertex distributions of neutrino events in the Far 
Detector fiducial volume. It is clear that the neutrino interactions are uniformly distributed in the FD fiducial region.

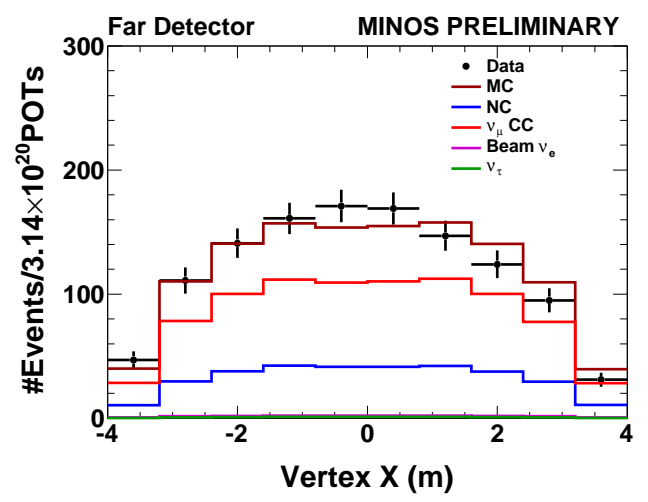

(a) Vertex $\mathrm{X}$

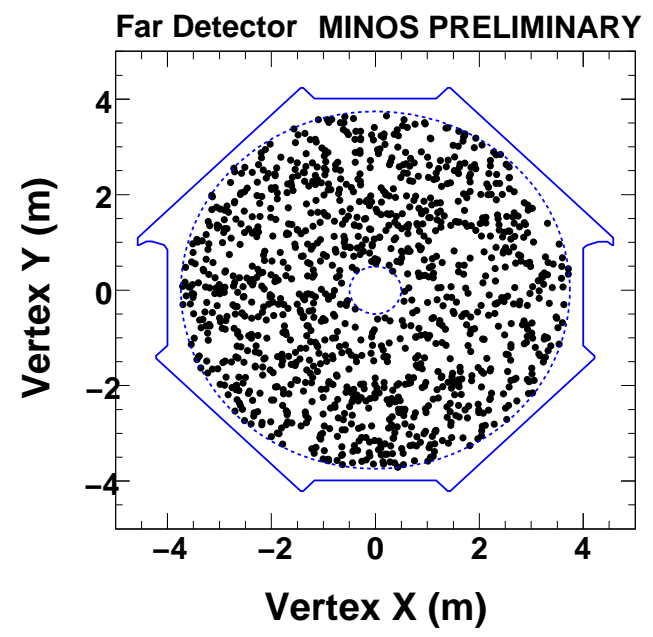

(c) Vertex X vs Y (data)

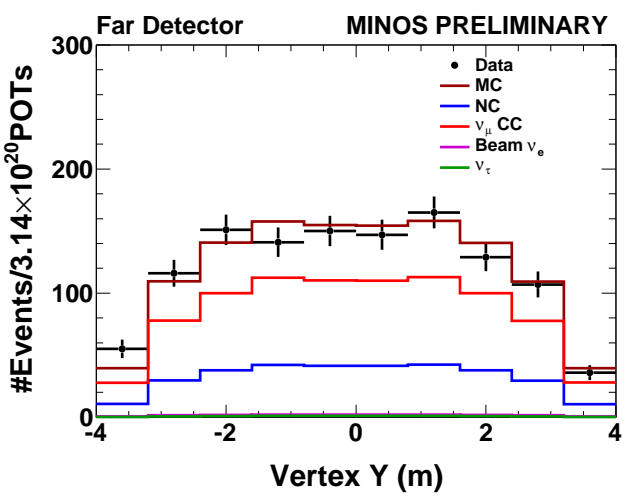

(b) Vertex Y

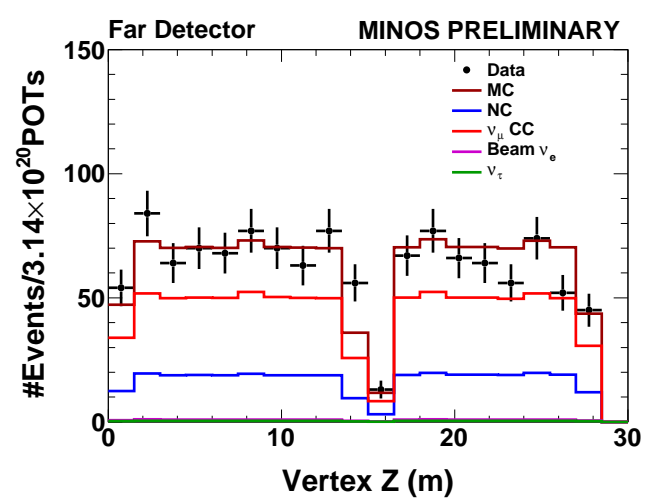

(d) Vertex Z

Figure 9.1: Reconstructed vertex distributions of neutrino events in the Far Detector fiducial volume.

Fig.9.2 shows the reconstructed track quantities after the fiducial volume cuts. Track-like planes are the "clean" hit planes in the track, i.e. ones that are free from the presence of non-track associated hits. Fig.9.3 shows the reconstructed shower quantities after the fiducial volume cuts. Because there can be multiple showers in 
the $\nu_{\mu} \mathrm{CC}$ events from bremsstrahlung radiation by the muon, we only consider the shower that is the closest to the event vertex ("primary shower" or "vertex shower"). The data and MC agreement is satisfactory for those reconstructed quantities.

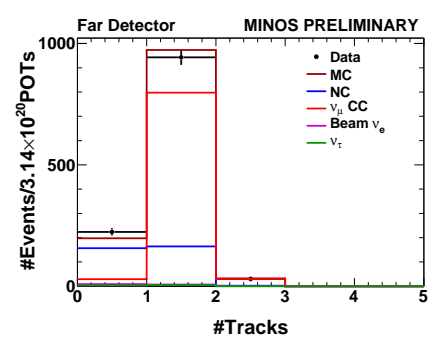

(a) Number of tracks

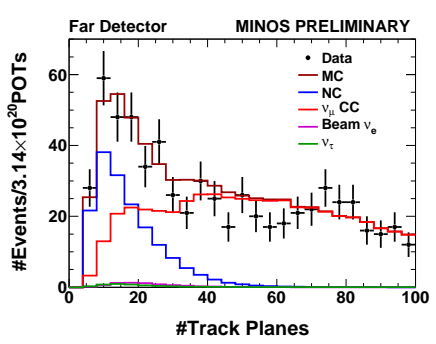

(b) Number of track planes

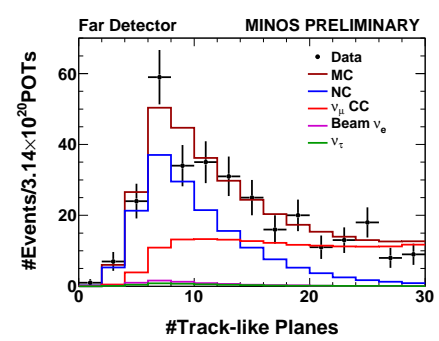

(c) Number of track-like planes

Figure 9.2: Reconstructed track quantities after fiducial volume cuts.

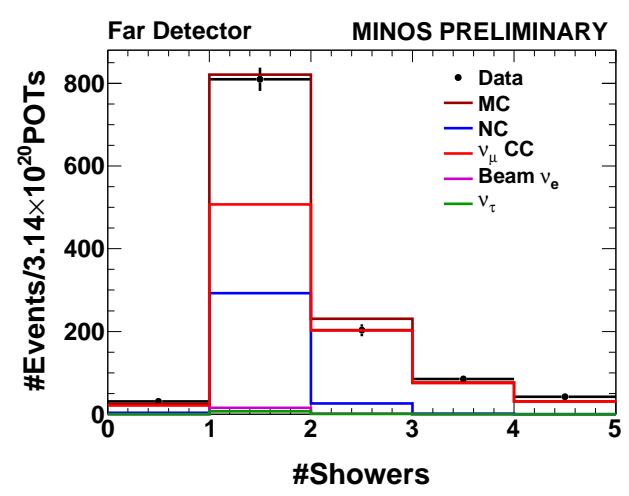

(a) Number of showers

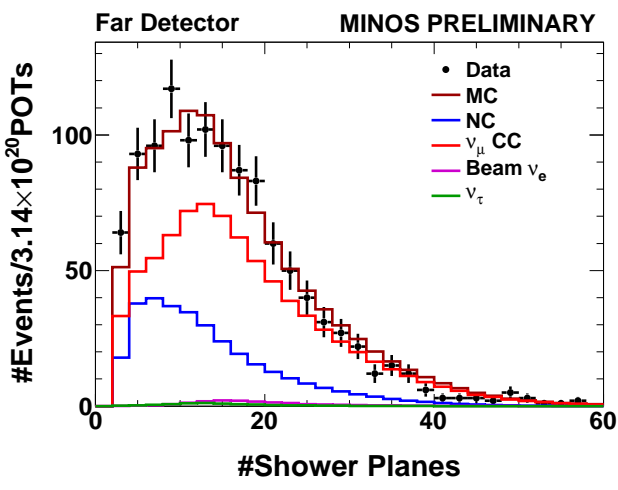

(b) Number of shower planes

Figure 9.3: Reconstructed shower quantities after fiducial volume cuts.

Fig.9.4 shows the distributions of two reconstructed kinematic quantities $Q^{2}$ and $W^{2}$ :

$$
\begin{aligned}
Q^{2} & =2 E_{\nu}\left(E_{\mu}-p_{\mu}^{L}\right)-m^{2} \\
W^{2} & =M^{2}+2 M \nu-Q^{2}
\end{aligned}
$$


where $E_{\nu}$ is the reconstructed neutrino energy, $E_{\mu}$ and $p_{\mu}^{L}$ are the reconstructed energy and longitudinal momentum of the muon, $M$ is the nucleon mass and $m$ is the muon mass. The MC reproduces data quite well. Note the calculated quantities are not meaningful for $\mathrm{NC}$ events since we can not measure the outgoing neutrinos in $\mathrm{NC}$ interactions and usually a pion track is mistaken as a muon track.

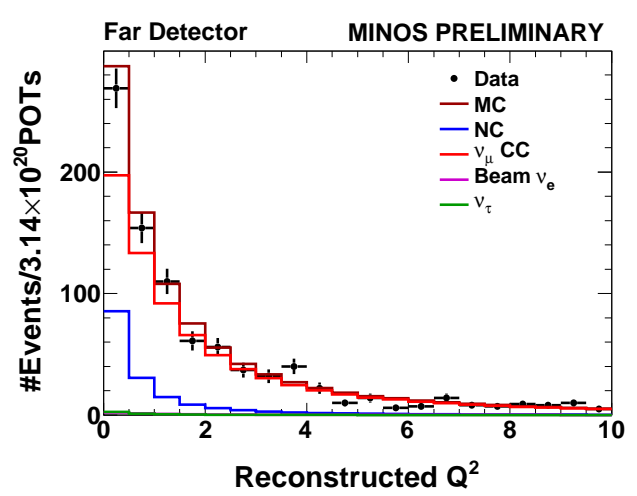

(a) Reconstructed $Q^{2}$

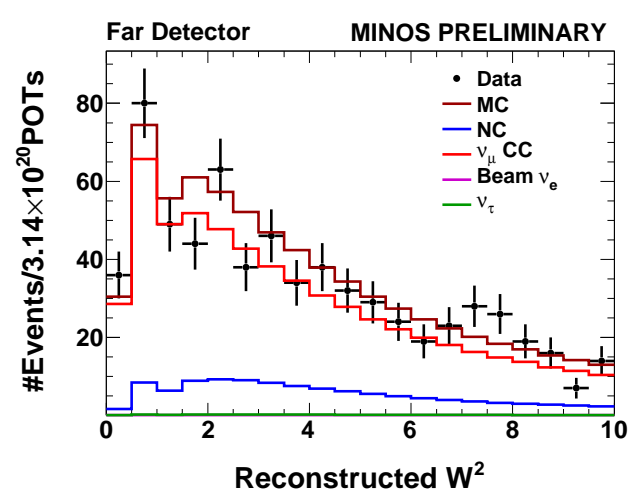

(b) Reconstructed $W^{2}$

Figure 9.4: Reconstructed $Q^{2}$ and $W^{2}$ after fiducial volume cuts.

\subsection{Pre-selection Cuts}

The next step in the analysis is removal of potential background events by a number of pre-selection cuts. We remove events that have long tracks (consistent with being $\nu_{\mu} \mathrm{CC}$ events) and poorly reconstructed events by requiring that accepted events satisfy satisfying the following criteria:

- Track length $<25$ planes.

- Track like length $<16$ planes.

- At least one reconstructed shower. 
- At least 5 contiguous planes with at least 0.5 MIP.

- Reconstructed energy between 1 and $8 \mathrm{GeV}$.

We apply a high energy cut $(E<8 G e V)$ since the potential $\nu_{\mu} \rightarrow \nu_{e}$ transition is highly suppressed at high energies so we are not interested in the high energy region. Furthermore this cut can remove half of the beam $\nu_{e}$ background which has a much broader energy spectrum than the signal events.

Table 9.1 showers the data and MC reduction as we apply various cuts step by step. This table demonstrates that the efficiencies for selecting data events and MC background events agree roughly within $2 \%$. Note the $\mathrm{MC}$ numbers do not have corrections based on ND data.

\begin{tabular}{|c|c|c|c|c|c|c|}
\hline Cuts & Data & Data Eff. & MC BG & BG Eff. & Potential Signal & Signal Eff. \\
\hline Fiducial Volume & 1188 & & 1196.4 & & 25.0 & \\
\hline Track planes $<25$ & 444 & $37.4 \%$ & 420.9 & $35.2 \%$ & 24.7 & $98.8 \%$ \\
\hline Track-like planes $<16$ & 410 & $34.5 \%$ & 385.9 & $32.3 \%$ & 24.4 & $97.6 \%$ \\
\hline \#showers $>0$ & 406 & $34.2 \%$ & 382.0 & $31.9 \%$ & 24.4 & $97.6 \%$ \\
\hline Cont.planes $>4$ & 286 & $24.1 \%$ & 277.0 & $23.2 \%$ & 21.5 & $86.0 \%$ \\
\hline E $>1.0 \mathrm{GeV}$ & 271 & $22.8 \%$ & 261.6 & $21.9 \%$ & 21.2 & $84.8 \%$ \\
\hline E $<8.0 \mathrm{GeV}$ & 227 & $19.1 \%$ & 208.9 & $17.5 \%$ & 20.4 & $81.6 \%$ \\
\hline
\end{tabular}

Table 9.1: FD data and MC reduction for $3.14 \times 10^{20}$ POTs. The standard MC was used to predict the number of background and potential signal events (i.e. no corrections based on ND data). Signal is computed at $\sin ^{2} 2 \theta_{13}=0.15$ (CHOOZ limit).

Fig.9.5 shows the distributions of the 11 variables used in the ANN after preselection cuts for data and background only MC. Fig.9.6 shows the ANN PID distributions for data, corrected background only MC and the raw background only MC. The correction to MC takes into account the data/MC differences in the ND PID distributions. Fig.9.7 shows the ANN PID distributions for data, corrected background 
only MC and background+signal MC.

\subsection{PID Cuts}

The final cut in the $\nu_{e}$ selection is a cut on the ANN PID. The optimal cut was found to be at 0.7 to give the maximum sensitivity. Before we opened the box on the signal region, we examined two different PID intervals outside the signal region. One is for PID value less than 0.55 (anti-PID sideband), the other is for PID value between 0.55 and 0.7. The results are summarized in Table 9.2. The number of

\begin{tabular}{|c|c|c|c|c|}
\hline Cuts & Data & Raw BG MC & Corrected BG Prediction & Potential Signal \\
\hline$P I D<0.55$ & 147 & 141.0 & $131.8 \pm 11.5($ stat. $) \pm 8.4($ sys. $)$ & 4.9 \\
\hline $0.55<P I D<0.7$ & 46 & 37.3 & $37.6 \pm 6.1($ stat. $) \pm 2.5($ sys. $)$ & 4.9 \\
\hline
\end{tabular}

Table 9.2: Comparison of the data selected in the two PID regions compared to the raw BG MC, the corrected background prediction, and the potential signal. Signal is computed at $\sin ^{2}\left(2 \theta_{13}\right)=0.15$ (CHOOZ limit). Numbers are for $3.14 \times 10^{20}$ POTs

background events expected in each PID region (corrected background prediction) is determined using the full ND data decomposition (horn on/off method described in Chapter 7) and $\mathrm{F} / \mathrm{N}$ extrapolation (described in Chapter 8). The $\sigma$ difference, defined as the difference between the data and background prediction divided by the combined statistical and systematic errors, is 1.1 for the region PID $<0.55$ and 1.3 for the region $0.55<P I D<0.7$. If there is a signal corresponding to $\theta_{13}$ at $\mathrm{CHOOZ}$ limit, the $\sigma$ differences are reduced to 0.7 and 0.5 respectively.

Table 9.3 summarizes the result of this MINOS $\nu_{e}$ analysis. We observe a total of 35 events in the signal region and we expect 27 events with the assumption of no $\nu_{e}$ appearance. This corresponds to a $1.4 \sigma$ excess using the combined statistical and systematic errors. If there is a signal corresponding to $\theta_{13}$ at $\mathrm{CHOOZ}$ limit, the $\sigma$ 

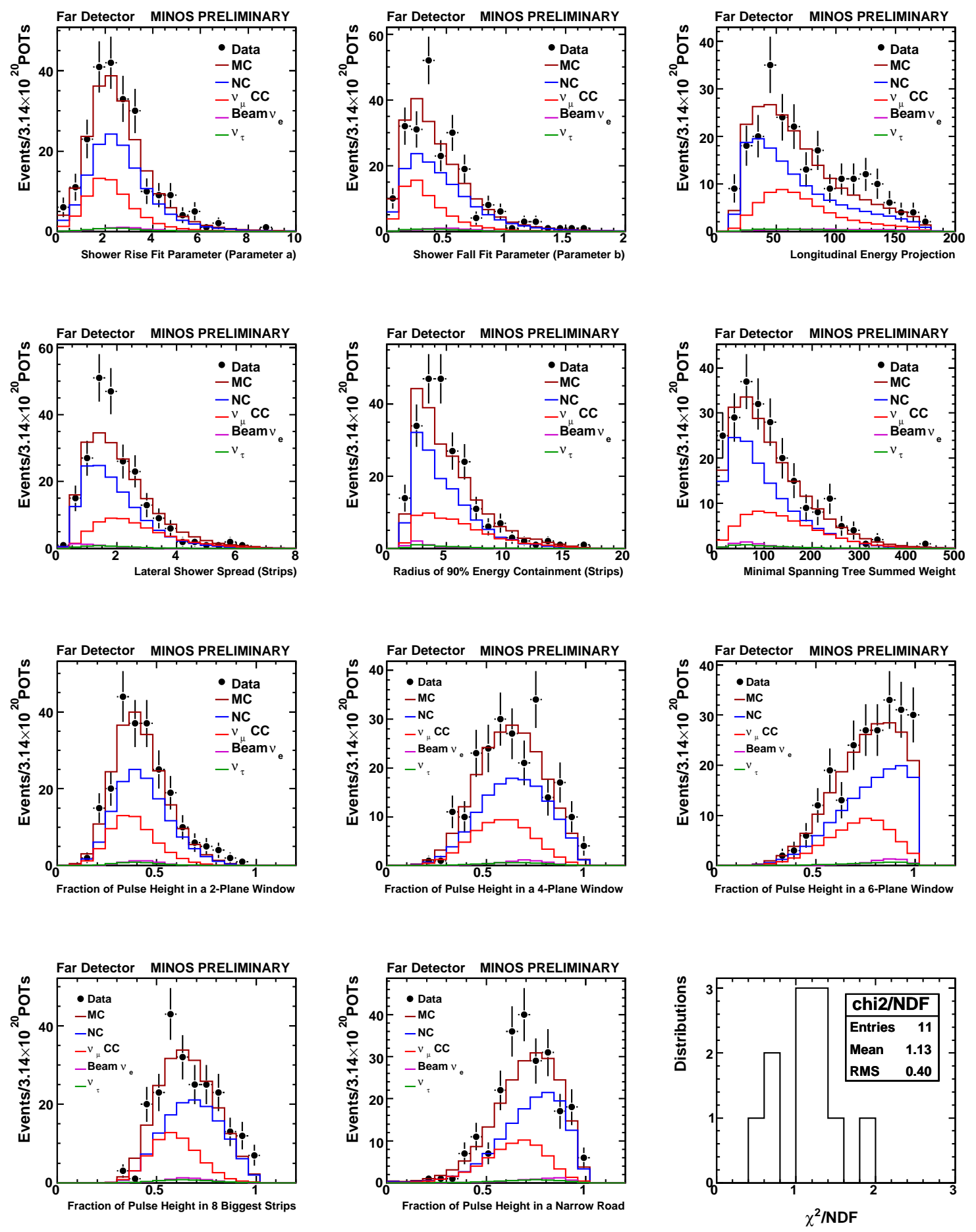

Figure 9.5: Distributions of the 11 variables used in the ANN after pre-selection cuts. The last plot shows the $\chi^{2} / \mathrm{NDF}$ of the 11 distributions. No ND data based correction was applied to MC. 


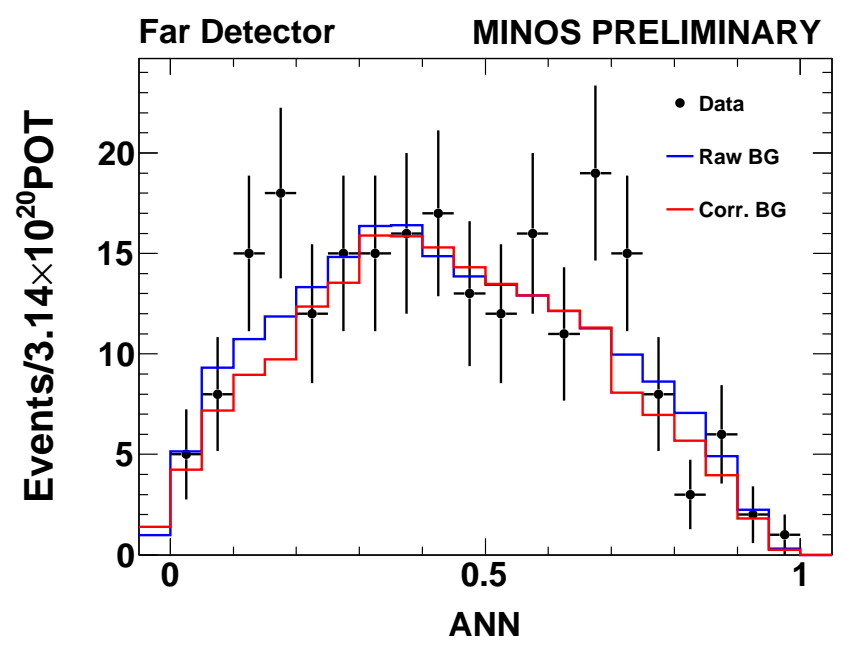

Figure 9.6: ANN PID distributions for data (black points), corrected background only $\mathrm{MC}$ (red) and the raw background only MC (blue). The bin below zero corresponds in an underflow bin which contains events with no assigned PID. The correction to $\mathrm{MC}$ takes into account the data/MC differences in the ND PID distributions.

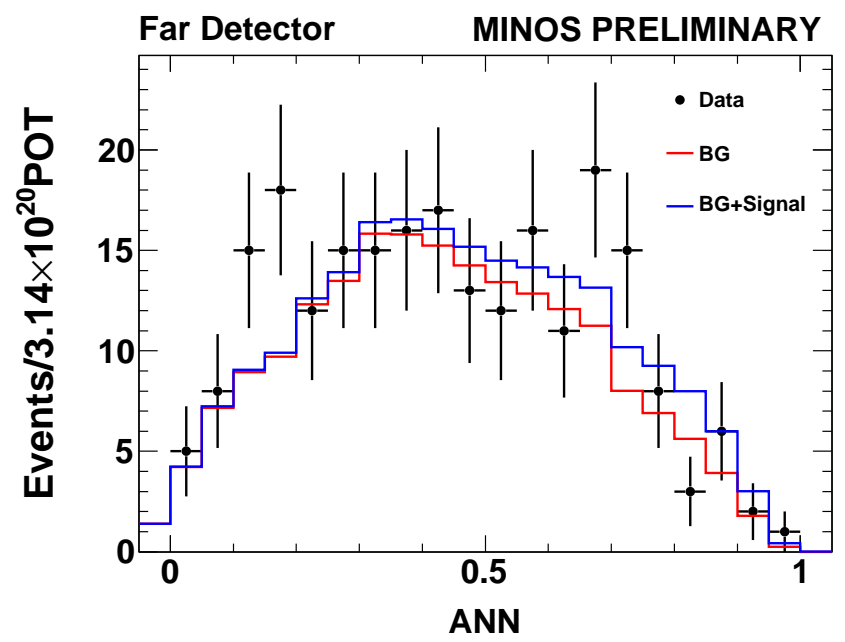

Figure 9.7: ANN PID distributions for data (black points), corrected background only MC (red) and background+signal MC (blue). Signal is computed at $\sin ^{2}\left(2 \theta_{13}\right)=0.15$ (CHOOZ limit). The bin below zero corresponds in an underflow bin which contains events with no assigned PID. 
difference is reduced to -0.4 .

\begin{tabular}{|c|c|c|c|c|}
\hline Cuts & Data & Raw BG MC & Corrected BG Prediction & Potential Signal \\
\hline PID $>0.7$ & 35 & 32.9 & $27.3 \pm 5.2($ stat. $) \pm 1.8($ sys. $)$ & $10.3 \pm 3.2($ stat. $) \pm 0.8($ sys. $)$ \\
\hline
\end{tabular}

Table 9.3: Comparison of the data selected in the signal regions compared to the raw $\mathrm{BG} \mathrm{MC}$, the corrected background prediction, and the potential signal. Signal is computed at $\sin ^{2}\left(2 \theta_{13}\right)=0.15$ (CHOOZ limit). Numbers are for $3.14 \times 10^{20}$ POTs

Fig.9.8 shows the spatial and temporal distributions of the 35 accepted data events. Despite low statistics, the events are consistent with being uniformly distributed in space and time.

Fig.9.9 shows the reconstructed visible energy of selected data compared to predictions. The left plot shows the comparison to the predicted background while the right plot shows the comparison to the predicted background + potential signal.

If we interpret the excess in the data as a signal, we can fit the data with a $\nu_{\mu} \rightarrow \nu_{e}$ oscillation hypothesis. We define the scaled $\chi^{2}$ in the same way as in the last chapter:

$$
\begin{aligned}
& \chi_{0}^{2}=2\left(N_{E x p}-N_{O b s}+N_{\text {Obs }} \ln \frac{N_{O b s}}{N_{E x p}}\right) \\
& \chi^{2}=\chi_{0}^{2} \times \frac{N_{E x p}}{\sigma_{\text {background }}^{2}+\sigma_{\text {signal }}^{2}+N_{E x p}}
\end{aligned}
$$

where $N_{O b s}$ is the observed number of events, $N_{E x p}$ is the expected number of events for a given set of oscillation parameters $\left(\theta_{13}, \delta_{C P}\right.$, etc. $), \sigma_{\text {background }}$ and $\sigma_{\text {signal }}$ are the systematic errors in the predicted background and signal events. The reason to choose this form of $\chi^{2}$ definition was discussed in Chapter 8 where we calculated the sensitivity.

Fig.9.10 shows the $\chi^{2}$ distributions as a function of $\sin ^{2}\left(2 \theta_{13}\right)$ and $\delta_{C P}$ for normal (left) and inverted (right) mass hierarchies. 


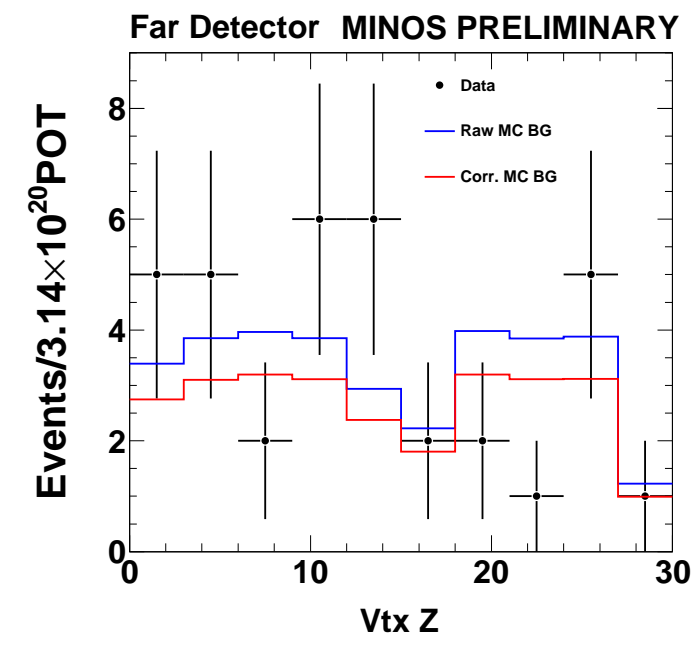

(a) Vertex z

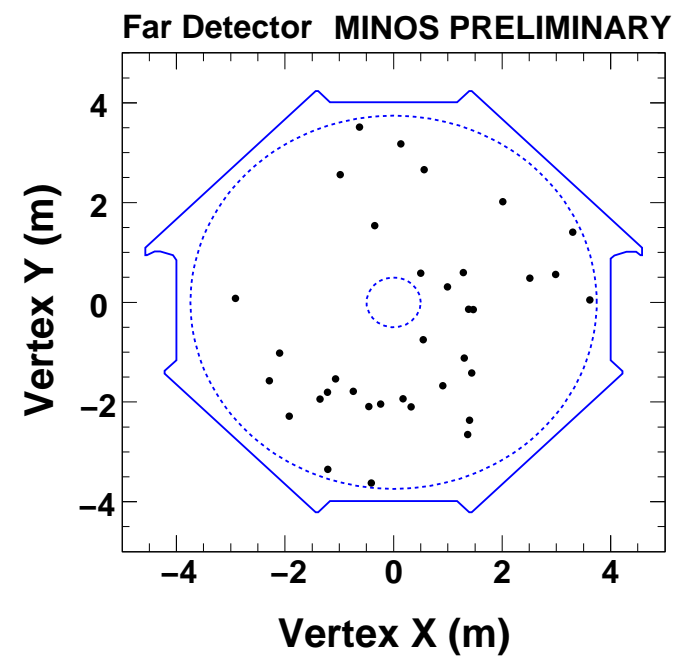

(b) Vertex x vs y

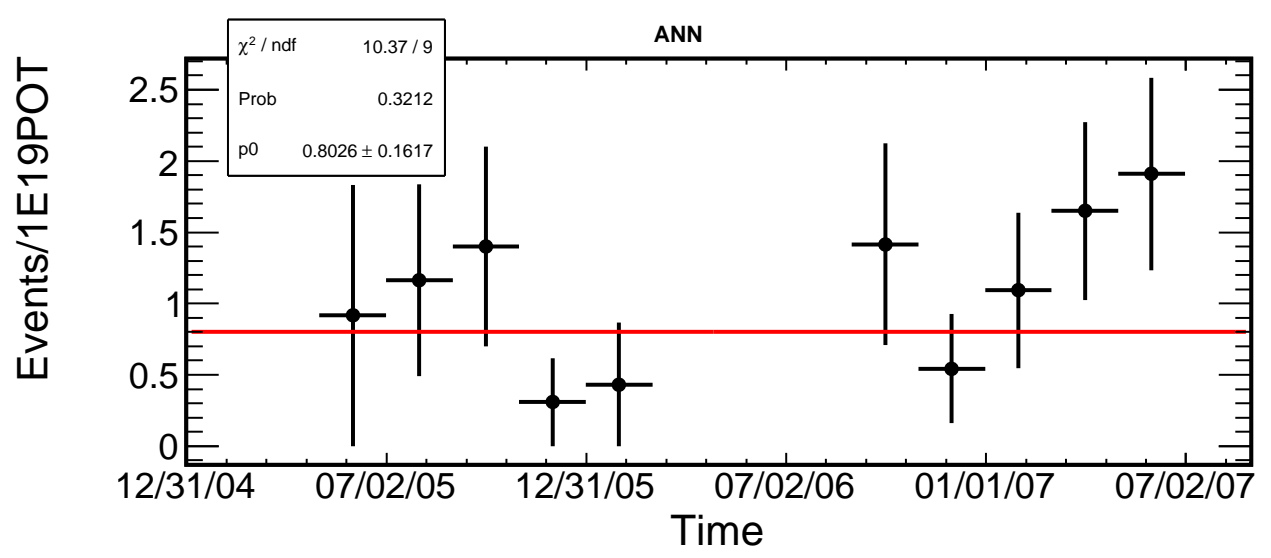

(c) Events per unit POT vs time

Figure 9.8: Distributions of events accepted by ANN (a) Vertex z; (b) Vertex x vs y; (c) Events per unit POT vs time (each bin corresponds to 2 month of data). 


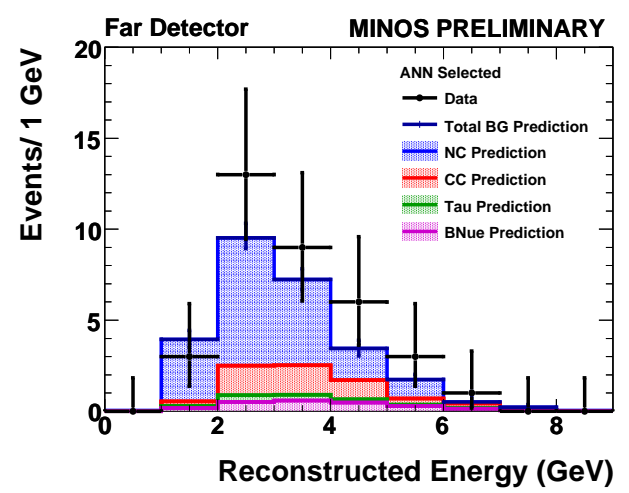

(a) Without signal prediction

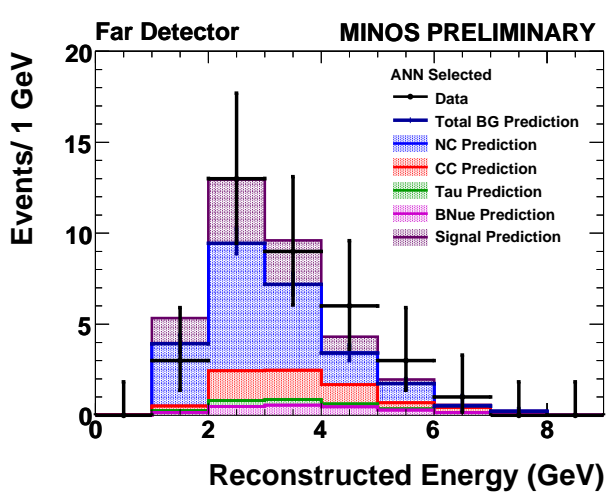

(b) With signal prediction

Figure 9.9: Reconstructed energy of selected data compared to predictions in the signal region. Signal is computed at $\sin ^{2}\left(2 \theta_{13}\right)=0.15$ (CHOOZ limit). Plots are stack plots.
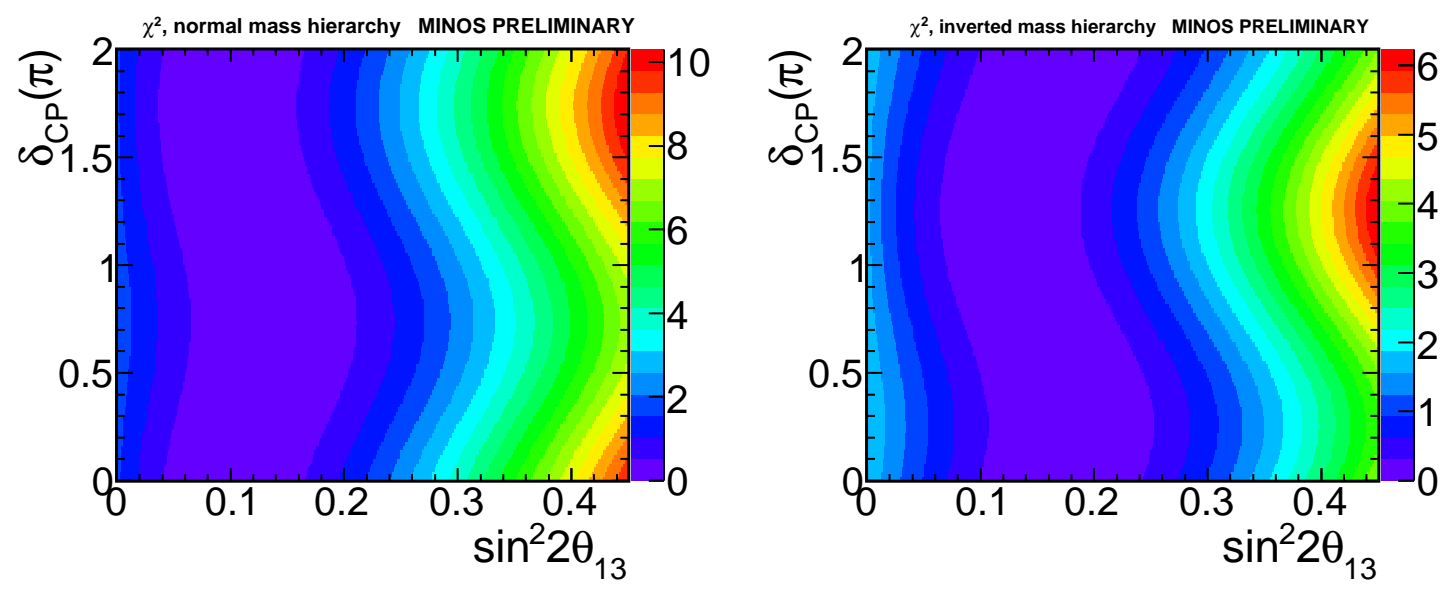

Figure 9.10: $\chi^{2}$ distributions as a function of $\sin ^{2}\left(2 \theta_{13}\right)$ and $\delta_{C P}$ for observation of 35 data events. Left: normal mass hierarchy $\left(\Delta m_{32}^{2}>0\right)$; Right: inverted mass hierarchy $\left(\Delta m_{32}^{2}<0\right)$. 
Fig.9.11 shows the 1 -d $\chi^{2}$ distributions if we assume $\delta_{C P}=0$. The matter effects have a large impact on the $\nu_{\mu} \rightarrow \nu_{e}$ oscillation as we discussed in Chapter 3 . For the normal (inverted) hierarchy, matter effects enhance (suppress) the transition probabilities for neutrinos. Therefore two curves are quite different for the two mass hierarchies.

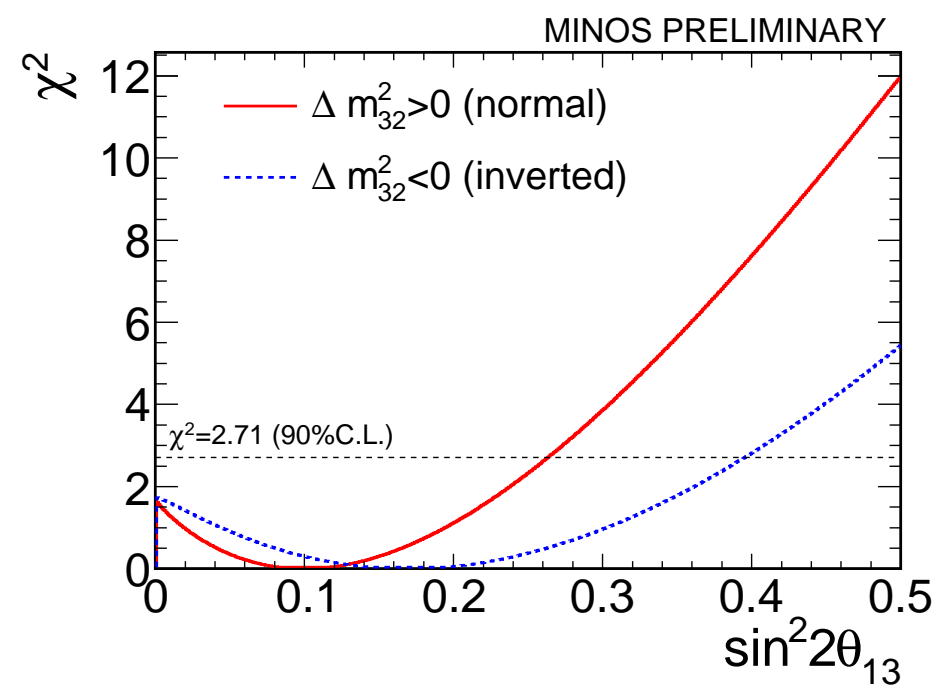

Figure 9.11: $\chi^{2}$ distributions as a function of $\sin ^{2}\left(2 \theta_{13}\right)$ for observation of data 35 events. We assume $\delta_{C P}=0$.

The best fit point oscillation values can be found by minimizing the $\chi^{2}$ value. The $90 \%$ limit is set at $\Delta \chi^{2}=2.71$. Fig.9.12 shows the best fit and $90 \%$ upper limit in $\sin ^{2} 2 \theta_{13}$ as a function of $\delta_{C P}$ for both mass hierarchies. 


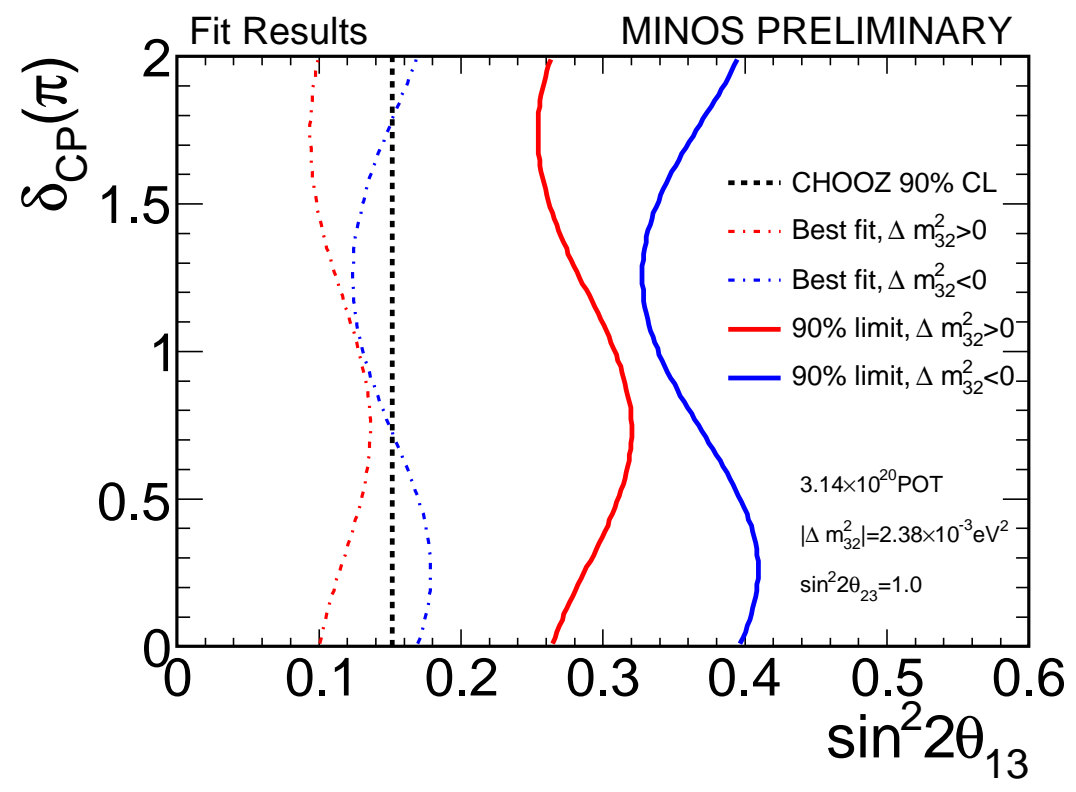

Figure 9.12: Best fit and 90\% upper limit in $\sin ^{2} 2 \theta_{13}$ as a function of $\delta_{C P}$ for the normal mass hierarchy (red) and the inverted mass hierarchy (blue) at the MINOS best fit value for $\Delta m_{32}^{2}$ and $\sin ^{2} 2 \theta_{23}$ for $3.14 \times 10^{20}$ POTs.

\subsection{Discussion of our results}

- If we ignore the matter effects, the oscillation probability for $\nu_{\mu} \rightarrow \nu_{e}$ transition can be written as

$$
\begin{aligned}
P\left(\nu_{\mu} \rightarrow \nu_{e}\right)= & \left|e^{-i\left(\Delta_{32}+\delta\right)} \sin 2 \theta_{13} \sin \theta_{23} \sin \Delta_{31}+\sin 2 \theta_{12} \cos \theta_{23} \cos \theta_{13} \sin \Delta_{21}\right|^{2} \\
= & \sin ^{2} \theta_{23} \sin ^{2} 2 \theta_{13} \sin ^{2} \Delta_{31}+\cos ^{2} \theta_{13} \cos ^{2} \theta_{23} \sin ^{2} 2 \theta_{12} \sin ^{2} \Delta_{21} \\
& +J_{r} \sin \Delta_{21} \sin \Delta_{31} \cos \left(\Delta_{32}+\delta\right) \\
= & P_{\text {atm }}+P_{\text {sol }}+P_{\text {int }}
\end{aligned}
$$

where $J_{r} \equiv \sin 2 \theta_{12} \sin 2 \theta_{23} \sin 2 \theta_{13} \cos \theta_{13}$ and $P_{a t m}, P_{\text {sol }}, P_{\text {int }}$ denote the atmospheric term (the leading term), the solar term, and the interference term. The symbol $\sin ^{2} 2 \theta_{13}$ in Figs $9.10,9.11$ and 9.12 is used as a shorthand for what we 
actually measure for a fixed mass hierarchy and $\delta_{C P}$, which is approximately $2 \sin ^{2} \theta_{23} \sin ^{2} 2 \theta_{13}$. This is important in comparing our results to those from reactor experiments, which actually do measure $\sin ^{2} \theta_{13}$, since, given the current limits on $\sin ^{2} 2 \theta_{23}, \sin ^{2} \theta_{23}$ can vary by a factor of 1.8 at $90 \%$ C.L..

- The $1.4 \sigma$ excess of data events can be a statistical fluctuation of background events. If we interpret the excess as a $\nu_{e}$ signal from $\nu_{\mu} \rightarrow \nu_{e}$ oscillation, the best fit $\sin ^{2} 2 \theta_{13}$ value is consistent with the $\mathrm{CHOOZ}$ limit. But we emphasize that our result is also consistent with $\theta_{13}=0$ at $90 \%$ C.L..

- In 2008, a global analysis of the combination of atmospheric, solar and longbaseline reactor neutrino data provided the global estimate $\sin ^{2} 2 \theta_{13}=0.06 \pm$ $0.04(1 \sigma)$, implying a preference for $\theta_{13}>0$ but with limited statistical significance ( $\sim 90 \%$ C.L.) [149]. Our result is also consistent with that analysis.

- The first MINOS $\nu_{e}$ analysis was done using data taken from 2005 to 2007 (3.14e20POT used in the analysis). The MINOS experiment is accumulating more data and is expected to more than double the data set by this summer. With increase statistics and improved understanding of systematic errors, we will definitely be able to improve the measurement of $\theta_{13}$.

- One thing worth noting is that we observed a $1.8 \sigma$ excess of data events compared with the prediction in our MRCC sideband, which is worrisome. We have done extensive tests but we did not find any mistakes in the analysis. Therefore we conclude the excess in the MRCC sideband is most likely due to statistical fluctuation. 


\section{Appendix A}

\section{Glossary of Acronyms}

ANN Artificial Neural Network

CC Charged Current interactions. If the neutrino type is not specified, CC usually refers to $\nu_{\mu} \mathrm{CC}$ background component in the $\nu_{e}$ analysis.

CalDet MINOS Calibration Detector

FD Far Detector

FOM Figure of Merit

MINOS Main Injector Neutrino Oscillation Search, a long baseline experiment sending neutrinos from Fermilab to Soudan, Minnesota.

MRCC Muon Removed $\nu_{\mu}$ Charged Current events. A sample to study the hadronic showers.

MRE Muon Removal with Electron addition. A sample with MC electrons and data (or MC) hadronic showers to mimic $\nu_{e} \mathrm{CC}$ events.

NC Neutral Current interactions 
ND Near Detector

NuMI Neutrinos at the Main Injector

PE Photoelectron

PID Particle IDentification, usually referred to as the output of the ANN in the $\nu_{e}$ analysis.

POT Proton on Target

PMT Photo Multiplier Tube 


\section{Bibliography}

[1] W. Pauli (1930) Letter to the Physical Society of Tübingen, unpublished; the letter is reproduced in L. M. Brown, Phys. Today 31N9, 23 (1978).

[2] J. Chadwick, Nature 129, 312 (1932).

[3] E. Fermi, Z. Phys. 88, 161 (1934).

[4] H. Bethe and R. Peierls, Nature 133, 532 (1934).

[5] F. Reines and C. L. Cowan, Phys. Rev. 92, 830 (1953); C. L. Cowan et al., Science 124, 103 (1956).

[6] B. Pontecorvo, Sov. Phys. JETP 6, 429 (1957) [Zh. Eksp. Teor. Fiz. 33, 549 (1957)].

[7] M. Goldhaber, L. Grodzins and A. W. Sunyar, Phys. Rev. 109, 1015 (1958).

[8] R. P. Feynman and M. Gell-Mann, Phys. Rev. 109, 193 (1958).

[9] G. Danby, J. M. Gaillard, K. Goulianos, L. M. Lederman, N. B. Mistry, M. Schwartz and J. Steinberger, Phys. Rev. Lett. 9, 36 (1962).

[10] M. L. Perl et al., Phys. Rev. Lett. 35, 1489 (1975).

[11] K. Kodama et al. [DONUT Collaboration], Phys. Lett. B 504, 218 (2001) [arXiv:hep-ex/0012035]. 
[12] F. J. Hasert et al. [Gargamelle Neutrino Collaboration], Phys. Lett. B 46, 138 (1973).

[13] S. L. Glashow, Nucl. Phys. 22, 579 (1961).

[14] A. Salam, in Elementary Particle Theory, N. Svartholm, ed.(Stockholm: Almquist and Wiksell, 1968).

[15] S. Weinberg, Phys. Rev. Lett. 19, 1264 (1967).

[16] G. Arnison et al. [UA1 Collaboration], Phys. Lett. B 122, 103 (1983).

[17] P. Bagnaia et al. [UA2 Collaboration], Phys. Lett. B 129, 130 (1983).

[18] R. Davis and D. S. Harmer, Bull. Am. Phys. Soc. 4, 217 (1959)

[19] R. J. Davis, D. S. Harmer and K. C. Hoffman, Phys. Rev. Lett. 20, 1205 (1968).

[20] Q. R. Ahmad et al. [SNO Collaboration], Phys. Rev. Lett. 87, 071301 (2001) [arXiv:nucl-ex/0106015].

[21] C. V. Achar et al., Phys. Lett. 18 (1965) 196.

[22] F. Reines et al., Phys. Rev. Lett. 15 (1965) 429.

[23] T. K. Gaisser, T. Stanev and G. Barr, Phys. Rev. D 38, 85 (1988).

[24] T. J. Haines et al., Phys. Rev. Lett. 57, 1986 (1986); D. Casper et al., Phys. Rev. Lett. 66, 2561 (1991); R. Becker-Szendy et al., Phys. Rev. D 46, 3720 (1992).

[25] K. S. Hirata et al. [KAMIOKANDE-II Collaboration], Phys. Lett. B 205, 416 (1988).

[26] M. H. Ahn et al. [K2K Collaboration], Phys. Rev. Lett. 90, 041801 (2003) [arXiv:hep-ex/0212007]. 
[27] K. Hirata et al. [KAMIOKANDE-II Collaboration], Phys. Rev. Lett. 58, 1490 (1987).

[28] G. S. Abrams et al., Phys. Rev. Lett. 63, 2173 (1989).

[29] D. Decamp et al. [ALEPH Collaboration], Phys. Lett. B 231, 519 (1989).

[30] G. 't Hooft, Nucl. Phys. B 33, 173 (1971), B 35, 167 (1971).

[31] B. Kayser, arXiv:hep-ph/0211134.

[32] Particle Data Group, W.-M. Yao et al., Journal of Physics, G 33, 1 (2006)

[33] B. Kayser, In the Proceedings of 32nd SLAC Summer Institute on Particle Physics (SSI 2004): Natures Greatest Puzzles, Menlo Park, California, 2-13 Aug 2004, pp L004 [arXiv:hep-ph/0506165].

[34] L. Stodolsky, Phys. Rev. D 58, 036006 (1998) [arXiv:hep-ph/9802387].

[35] Z. Maki, M. Nakagawa and S. Sakata, Prog. Theor. Phys. 28, 870 (1962).

[36] B. Pontecorvo, Sov. Phys. JETP 26, 984 (1968) [Zh. Eksp. Teor. Fiz. 53, 1717 (1967)].

[37] S. Brice, arXiv:0711.2988 [hep-ex].

[38] B. T. Cleveland et al., Astrophys. J. 496, 505 (1998).

[39] K. S. Hirata et al. [KAMIOKANDE-II Collaboration], Phys. Rev. Lett. 63, 16 (1989).

[40] B. Aharmim et al. [SNO Collaboration], Phys. Rev. C 72, 055502 (2005) [arXiv:nucl-ex/0502021]. 
[41] J. Hosaka et al. [Super-Kamkiokande Collaboration], Phys. Rev. D 73, 112001 (2006) [arXiv:hep-ex/0508053].

[42] P. Anselmann et al. [GALLEX Collaboration], Phys. Lett. B 285, 376 (1992).

[43] D. N. Abdurashitov et al., Phys. Lett. B 328, 234 (1994).

[44] M. Altmann et al. [GNO COLLABORATION Collaboration], Phys. Lett. B 616, 174 (2005) [arXiv:hep-ex/0504037].

[45] C. Arpesella et al. [The Borexino Collaboration], Phys. Rev. Lett. 101, 091302 (2008) [arXiv:0805.3843 [astro-ph]].

[46] T. Araki et al. [KamLAND Collaboration], Phys. Rev. Lett. 94, 081801 (2005) [arXiv:hep-ex/0406035].

[47] R. Becker-Szendy et al., Phys. Rev. D 46, 3720 (1992).

[48] Y. Fukuda et al. [Kamiokande Collaboration], Phys. Lett. B 335, 237 (1994).

[49] W. A. Mann, in Proc. of the 19th Intl. Symp. on Photon and Lepton Interactions at High Energy LP99 ed. J.A. Jaros and M.E. Peskin, In the Proceedings of 19th International Symposium on Lepton and Photon Interactions at HighEnergies (LP 99), Stanford, California, 9-14 Aug 1999, pp 229-256 [arXiv:hepex/9912007].

[50] Y. Fukuda et al. [Super-Kamiokande Collaboration], Phys. Rev. Lett. 81, 1562 (1998) [arXiv:hep-ex/9807003].

[51] C. B. Smith, Calibration of the MINOS detectors and extraction of neutrino oscillation parameters, Ph.D. Thesis, University Coll. London (2002), FERMILABTHESIS-2002-58 
[52] K. Scholberg [Super-Kamiokande Collaboration], arXiv:hep-ex/9905016.

[53] Y. Ashie et al. [Super-Kamiokande Collaboration], Phys. Rev. D 71, 112005 (2005) [arXiv:hep-ex/0501064].

[54] Y. Ashie et al. [Super-Kamiokande Collaboration], Phys. Rev. Lett. 93, 101801 (2004) [arXiv:hep-ex/0404034].

[55] E. Aliu et al. [K2K Collaboration], Phys. Rev. Lett. 94, 081802 (2005) [arXiv:hep-ex/0411038].

[56] M. H. Ahn et al. [K2K Collaboration], Phys. Rev. D 74, 072003 (2006) [arXiv:hep-ex/0606032].

[57] D. G. Michael et al. [MINOS Collaboration], Phys. Rev. Lett. 97, 191801 (2006) [arXiv:hep-ex/0607088].

[58] P. Adamson et al. [MINOS Collaboration], Phys. Rev. D 77, 072002 (2008) arXiv:0711.0769 [hep-ex].

[59] P. Adamson et al. [MINOS Collaboration], Phys. Rev. Lett. 101, 131802 (2008) [arXiv:0806.2237 [hep-ex]].

[60] M. Guler et al. [OPERA Collaboration], "OPERA: An appearance experiment to search for $\nu_{\mu} \leftrightarrow \nu_{\tau}$ oscillations in the CNGS beam. Experimental proposal."

[61] M. Apollonio et al. [CHOOZ Collaboration], Phys. Lett. B 466, 415 (1999) [arXiv:hep-ex/9907037].

[62] F. Boehm et al., Phys. Rev. D 64, 112001 (2001) [arXiv:hep-ex/0107009].

[63] M. Maltoni, T. Schwetz, M. A. Tortola and J. W. F. Valle, Phys. Rev. D 68, 113010 (2003) [arXiv:hep-ph/0309130]. 
[64] M. Maltoni, T. Schwetz, M. A. Tortola and J. W. F. Valle, New J. Phys. 6, 122 (2004) [arXiv:hep-ph/0405172].

[65] S. Goswami and A. Y. Smirnov, Phys. Rev. D 72, 053011 (2005) [arXiv:hep$\mathrm{ph} / 0411359]$.

[66] T. Schwetz, AIP Conf. Proc. 981, 8 (2008) [arXiv:0710.5027 [hep-ph]].

[67] F. Ardellier et al. [Double Chooz Collaboration], arXiv:hep-ex/0606025.

[68] X. Guo et al. [Daya Bay Collaboration], arXiv:hep-ex/0701029.

[69] J. C. Anjos et al., Nucl. Phys. Proc. Suppl. 155, 231 (2006) [arXiv:hepex/0511059].

[70] K. K. Joo [RENO Collaboration], Nucl. Phys. Proc. Suppl. 168, 125 (2007).

[71] D. S. Ayres et al. [NOvA Collaboration], arXiv:hep-ex/0503053.

[72] Y. Itow et al. [The T2K Collaboration], arXiv:hep-ex/0106019.

[73] A. Aguilar et al. [LSND Collaboration], Phys. Rev. D 64, 112007 (2001) [arXiv:hep-ex/0104049].

[74] A. A. Aguilar-Arevalo et al. [The MiniBooNE Collaboration], Phys. Rev. Lett. 98, 231801 (2007) [arXiv:0704.1500 [hep-ex]].

[75] B. Armbruster et al. [KARMEN Collaboration], Phys. Rev. D 65, 112001 (2002) [arXiv:hep-ex/0203021].

[76] Y. Declais et al., Nucl. Phys. B 434, 503 (1995).

[77] C. Kraus et al., Eur. Phys. J. C 33, S805 (2004).

[78] K. Assamagan et al., Phys. Rev. D 53, 6065 (1996). 
[79] J. M. Roney, Nucl. Phys. Proc. Suppl. 91, 287 (2001).

[80] L. Wolfenstein, Phys. Rev. D 17, 2369 (1978).

[81] S. P. Mikheev and A. Y. Smirnov, Sov. J. Nucl. Phys. 42, 913 (1985) [Yad. Fiz. 42, $1441(1985)]$.

[82] S. P. Mikheev and A. Y. Smirnov, Nuovo Cim. C 9, 17 (1986).

[83] P. F. Harrison, D. H. Perkins and W. G. Scott, Phys. Lett. B 530, 167 (2002) [arXiv:hep-ph/0202074].

[84] P. F. Harrison and W. G. Scott, Phys. Lett. B 535, 163 (2002) [arXiv:hepph/0203209]. P. F. Harrison and W. G. Scott, Phys. Lett. B 557, 76 (2003) [arXiv:hep-ph/0302025]. Z. z. Xing, Phys. Lett. B 533, 85 (2002) [arXiv:hep$\mathrm{ph} / 0204049]$.

[85] R. M. Zwaska, Accelerator systems and instrumentation for the NuMI neutrino beam, Ph.D. Thesis, University of Texas at Austin (2005), FERMILAB-THESIS2005-73

[86] C. C. Jensen and G. A. Krafczyk, In the Proceedings of Particle Accelerator Conference (PAC 05), Knoxville, Tennessee, 16-20 May 2005, pp 692.

[87] S. Shukla, J. Marriner and J. Griffin, In the Proceedings of 1996 DPF / DPB Summer Study on New Directions for High-Energy Physics (Snowmass 96), Snowmass, Colorado, 25 Jun - 12 Jul 1996, pp ACC015.

[88] R. Zwaska et al., Nucl. Instrum. Meth. A 568, 548 (2006) [arXiv:physics/0609106].

[89] D. G. Michael [MINOS Collaboration], arXiv:0805.3170 [physics.ins-det]. 
[90] J. Oliver, N. Felt, G. Feldman, A. Lebedev and R. Lee, IEEE Trans. Nucl. Sci. 51, 2193 (2004).

[91] T. Cundiff et al., IEEE Trans. Nucl. Sci. 53, 1347 (2006).

[92] Calibration Position Paper, MINOS-doc-3137-v3

[93] GPS Timing Receiver XL-AK by Symetricon (formerly TrueTime), 3750 Westwind Blvd., Santa Rosa, CA 95403, U.S.A.).

[94] P. Adamson et al., Nucl. Instrum. Meth. A 492, 325 (2002) [arXiv:hepex/0204021].

[95] P. Ward, Far Detector Linearity Calibration, MINOS-doc-1886-v1

[96] P. Adamson et al., Nucl. Instrum. Meth. A 556, 119 (2006).

[97] P. L. Vahle, Electromagnetic interactions in the MINOS detectors, Ph.D. thesis, University of Texas at Austin (2004), Fermilab-Thesis-2004-35.

[98] M. A. Kordosky, Hadronic interactions in the MINOS detectors, Ph.D. thesis, University of Texas at Austin (2004), FERMILAB-THESIS-2004-34

[99] A. Fasso', A. Ferrari, J. Ranft, and P.R. Sala, FLUKA: a multi-particle transport code, CERN-2005-10 (2005), INFN/TC_05/11, SLAC-R-773

A. Fasso', A. Ferrari, S. Roesler, P.R. Sala, G. Battistoni, F. Cerutti, E. Gadioli, M.V. Garzelli, F. Ballarini, A. Ottolenghi, A. Empl and J. Ranft, The physics models of FLUKA: status and recent developments, Computing in High Energy and Nuclear Physics 2003 Conference (CHEP2003), La Jolla, CA, USA, March 24-28, 2003, (paper MOMT005), eConf C0303241 (2003), arXiv:hep-ph/0306267

[100] R. Brun et al., GEANT Detector Description and Simulation Tool, CERN Program Library Long Writeup W5013 (1994). 
[101] H. Gallagher, Nucl. Phys. Proc. Suppl. 112, 188 (2002).

[102] D. Rein and L. M. Sehgal, Annals Phys. 133, 79 (1981).

[103] A. Bodek and U. K. Yang, AIP Conf. Proc. 721, 358 (2004).

[104] T. Yang, C. Andreopoulos, H. Gallagher and P. Kehayias, AIP Conf. Proc. 967, 269 (2007).

[105] T. Sjostrand, S. Mrenna and P. Skands, JHEP 0605, 026 (2006) [arXiv:hep$\mathrm{ph} / 0603175]$.

[106] R. D. Ransome, Nucl. Phys. Proc. Suppl. 139, 208 (2005).

[107] C. Zeitnitz and T. A. Gabriel, Nucl. Instrum. Meth. A 349, 106 (1994).

[108] M. Szleper and A. Para, arXiv:hep-ex/0110001.

[109] P. Adamson et al. [MINOS Collaboration], Phys. Rev. Lett. 101, 221804 (2008) [arXiv:0807.2424 [hep-ex]].

[110] S. Abe et al. [KamLAND Collaboration], arXiv:0801.4589 [hep-ex].

[111] M. Apollonio et al. [CHOOZ Collaboration], Eur. Phys. J. C 27, 331 (2003) [arXiv:hep-ex/0301017].

[112] E. K. Akhmedov, R. Johansson, M. Lindner, T. Ohlsson and T. Schwetz, JHEP 0404, 078 (2004) [arXiv:hep-ph/0402175].

[113] E. Longo and I. Sestili, Nucl. Instrum. Meth. 128, 283 (1975) [Erratum-ibid. 135, $587(1976)]$.

[114] W. M. Yao et al. [Particle Data Group], J. Phys. G 33, 1 (2006).

[115] B. Rossi, High Energy Particles, Prentice-Hall, Inc., Englewood Cliffs, NJ, 1952. 
[116] W.R. Nelson, H. Hirayama, and D.W.O. Rogers, The EGS4 Code System, SLAC-265, Stanford Linear Accelerator Center (Dec. 1985).

[117] A. Hocker et al., arXiv:physics/0703039.

[118] C. Andreopoulos [GENIE Collaboration], Acta Phys. Polon. B 37, 2349 (2006); Nucl. Phys. Proc. Suppl. 159, 217 (2006); http://www.genie-mc.org

[119] Many experiments have demonstrated that the ratio of average $\pi$ multiplicities $\frac{2\left\langle n_{\pi^{0}}\right\rangle}{\left\langle n_{\pi^{+}}\right\rangle\left\langle n_{\pi^{-}}\right\rangle}$is consistent with 1, e.g. W. Wittek et al. [BEBC WA59 Collaboration], Z. Phys. C 40, 231 (1988).

[120] Z. Koba, H. B. Nielsen and P. Olesen, Nucl. Phys. B 40, 317 (1972).

[121] M. Derrick et al., Phys. Rev. D 17, 1 (1978).

[122] Cooper el al., Neutrino 1982 proceedings.

[123] F. James, Monte Carlo Phase Space, CERN 68-15 (1968)

[124] A. B. Clegg and A. Donnachie, Z. Phys. C 13, 71 (1982).

[125] B. Andersson, G. Gustafson, G. Ingelman and T. Sjostrand, Phys. Rept. 97, $31(1983)$.

[126] A. Rubbia, "NUX-neutrino generator", 1st Workshop on Neutrino - Nucleus Interactions in the Few GeV Region (NuInt01), Tsukuba, Japan, 13-16 Dec 2001. http://neutrino.kek.jp/nuint01/slide/Rubbia.1.pdf

[127] R.S. Gilmore, Single Particle Detection and Measurement, CRC Press, 1992.

[128] D. Zieminska et al., Phys. Rev. D 27, 47 (1983). 
[129] S. Barlag et al. [Amsterdam-Bologna-Padua-Pisa-Saclay-Turin Collaboration], Z. Phys. C 11, 283 (1982) [Erratum-ibid. C 14, 281 (1982)].

[130] P. Allen et al. [Aachen-Bonn-CERN-Munich-Oxford Collaboration], Nucl. Phys. B 181, 385 (1981).

[131] A. A. Ivanilov et al., Yad. Fiz. 41, 1520 (1985) [Sov. J. Nucl. Phys. 41, 963 (1985)].

[132] H. Grassler et al. [Aachen-Birmingham-Bonn-CERN-London-Munich-Oxford Collaboration], Nucl. Phys. B 223, 269 (1983).

[133] V. Ammosov et al., Nuovo Cim. A 51, 539 (1979).

[134] D. Allasia et al. [AMSTERDAM-BERGEN-BOLOGNA-PADUA-PISASACLAY-TURIN COLLABORATION Collaboration], Z. Phys. C 24, 119 (1984).

[135] D. Allasia et al. [Amsterdam-Bergen-Bologna-Padua-Pisa-Saclay-Turin Collaboration], Z. Phys. C 27, 239 (1985).

[136] J. P. Berge et al., Phys. Rev. D 18, 3905 (1978).

[137] M. Derrick et al., Phys. Rev. D 24, 1071 (1981) [Erratum-ibid. D 30, 1129 (1984)].

[138] W.A. Mann, private communication.

[139] R. Merenyi et al., Phys. Rev. D 45, 743 (1992).

[140] S. Dytman and H. Gallagher, New Intranuke model for Neugen - hA, MINOSdoc-3484-v1

S. Dytman and H. Gallagher, Changes to the Determination of the Mean Free Path of Hadrons in Nuclear Matter in INTRANUKE, MINOS-doc-4358-v2 
[141] V. Ammosov, "Low multiplicity final states in neutrino interaction at low energy - SKAT results", 1st Workshop on Neutrino - Nucleus Interactions in the Few GeV Region (NuInt01), Tsukuba, Japan, 13-16 Dec 2001. http://neutrino.kek.jp/nuint01/slide/ammosov.pdf

[142] R. Ospanov, Selecting charged current $\nu_{\mu}$ events in the data and the simulation, MINOS-doc-4390-v1

[143] K. Lang et al., Nucl. Instrum. Meth. A 545, 852 (2005).

[144] M. Bardadin-Otwinowska, L. Michejda, S. Otwinowski and R. Sosnowski Phys. Lett. 21, 351 (1966).

[145] H. Gallagher et al., The Horn On/Off Method and Physics Simulations Uncertainties, MINOS-doc-5413-v1

[146] J. Boehm et al., Systematic Uncertainties on the Measurement of $\nu_{e}$ CC Appearance, MINOS-doc-5387-v6

[147] J. Boehm, Measuring $\nu_{e}$ CC Selection Efficiency and Systematic Error using the Muon Removal with MC Electron Addition Process, MINOS-doc-5385-v4

[148] J. Boehm, Far Detector Background Prediction and Sensitivities, MINOS-doc4117-v9

[149] G. L. Fogli, E. Lisi, A. Marrone, A. Palazzo and A. M. Rotunno, Phys. Rev. Lett. 101, 141801 (2008) [arXiv:0806.2649 [hep-ph]]. 